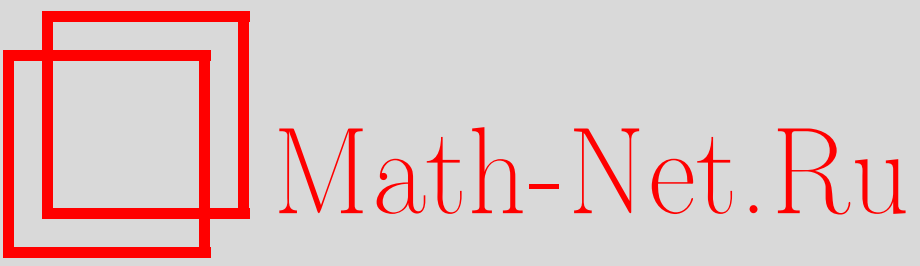

В. Г. Кановей, В. А. Любецкий, О некоторых классических проблемах дескриптивной теории множеств, УМН, 2003, том 58, выпуск 5, 3-88

DOI: https://doi.org/10.4213/rm666

Использование Общероссийского математического портала Math-Net.Ru подразумевает, что вы прочитали и согласны с пользовательским соглашением

http://www.mathnet.ru/rus/agreement

Параметры загрузки:

IP: 54.198 .64 .247

26 апреля 2023 г., 13:18:19 


\title{
О НЕКОТОРЫХ КЛАССИЧЕСКИХ ПРОБЛЕМАХ ДЕСКРИПТИВНОЙ ТЕОРИИ МНОЖЕСТВ
}

\author{
В. Г. КАНОВЕЙ, В. А. ЛЮБЕЦКИЙ
}

\begin{abstract}
100-летие со дня рождения П. С. Новикова дает волнующий повод изложить с современной точки зрения и с полными доказательствами, по-видимому, окончательные решения тех классических проблем о свойствах регулярности точечных множеств, которые были сформулированы Лузиным, а в некоторой мере еще раньше Адамаром, Борелем, Лебегом, и относятся к дескриптивной теории множеств. Решения этих проблем начались с пионерских работ Александрова, Суслина, Лузина 1916-17 годов, а затем были продвинуты в фундаментальных исследованиях Гёделя, Новикова, Коэна и их последователей. Это направление в математике отличается тем, что, с одной стороны, является обычной математической теорией о естественных свойствах точечных множеств и функций, далекой от общей теории множеств или таких внутренних проблем математической логики, как, например, непротиворечивость или теоремы Гёделя, а с другой стороны, оно стало местом приложения наиболее тонких средств современной математической логики.

Библиография: 97 названий.
\end{abstract}

\section{СОДЕРЖАНИЕ}

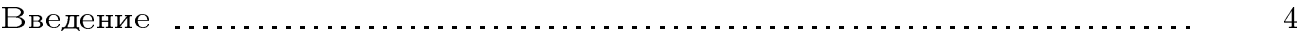

$\S 1$. Борелевские и проективные множества $\ldots \ldots \ldots \ldots \ldots \ldots \ldots \ldots \ldots \ldots \ldots . . \ldots \ldots$

1А. Вещественная прямая, польские пространства и бэровское пространство 10

1Б. Основные свойства регулярности в польских пространствах . . . . . . . . . 12

1В. Проективная иерархия и язьк аналитических формул . . . . . . . . . . . . . 14

1 Г. Элементы теории $\Pi_{1}^{1}$-множеств ............................ 16

1Д. Отступление: кодировка точками бэровского пространства . . . . . . . . . 18

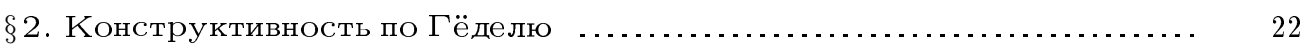

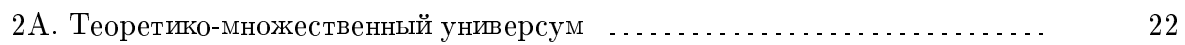

2Б. Общее понятие конструктивности по Гёделю .................... 23

2В. Конструктивность в области бэровского пространства .............. 25

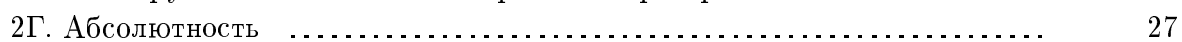

$\S 3$. Резольвенты классических проблем: первая часть $\ldots . \ldots \ldots \ldots \ldots \ldots . . . . . . . .29$

3А. Коэновские и случайные точки . . . . . . . . . . . . . . . . . . . . . . . . 29

3Б. Главные резултаты о резолвентах свойств регулярности ......... 30

3В. Несчетные $\Pi_{1}^{1}$-множества без совершенного ядра ................. 31

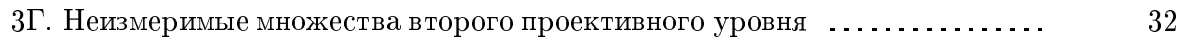

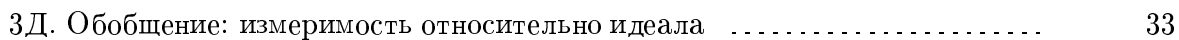


3Е. Множества, неизмеримые относительно идеала

3Ж. Непротиворечивость существования таких множеств ............. 36

$\S 4$. Резольвенты классических проблем: вторая часть ................ 38

4А. Основы форсинга …

4Б. Если конструктивных точек мало, то $\Pi_{1}^{1}$-множества имеют свойство со-

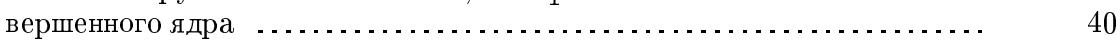

4В. Форсинг, порожденный идеалом борелевских множеств . ............. 42

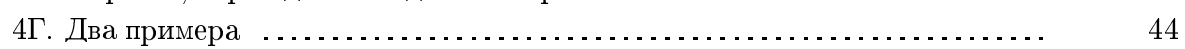

4Д. Если неслучайных точек мало, то все множества второго проективного

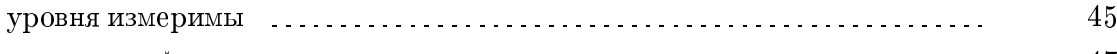

$\S 5$. Комбинаторика финального доминирования ................... 47

5А. Финальное доминирование и измеримость ..................... 48

5Б. Финальное доминирование и свойство Бэра .................... 50

5В. В классе $\Sigma_{2}^{1}$ измеримость влечет свойство Бэра ................. 51

§6. "Элементарное" доказательство одной из теорем . . . . . . . . . . . . 53

6А. Анализ ее доказательства . . . . . . . . . . . . . .

6Б. Дескриптивные континуумы …................................. 54

6В. Генерические расширения дескриптивных континуумов ............. 55

6 . Окончание "элементарного" доказательства теоремы $6.1 \ldots \ldots \ldots \ldots . .58$

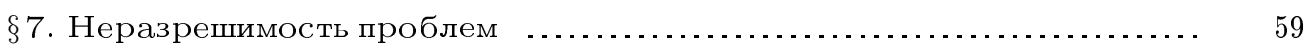

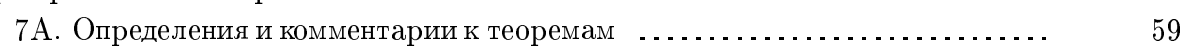

7Б. Первая модель - одна склеивающая функция … . . . . . . . . . . . . . 61

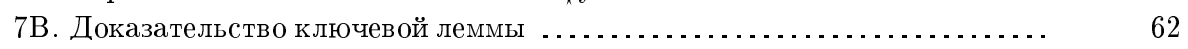

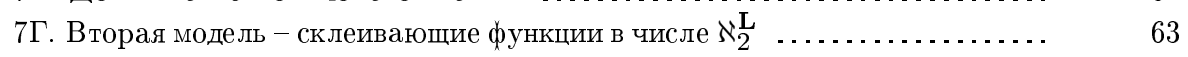

7.Д. Свойство совершенного ядра во второй модели .................. 65

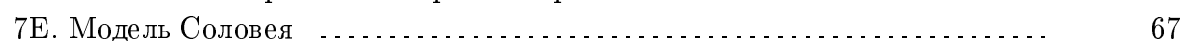

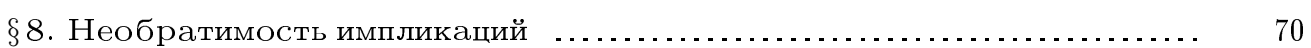

8А. Четыре ключевых форсинга ................................. 70

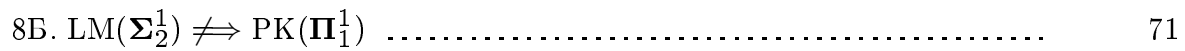

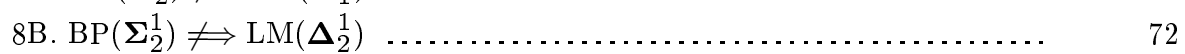

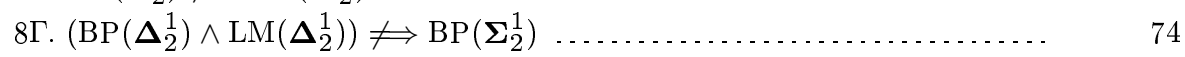

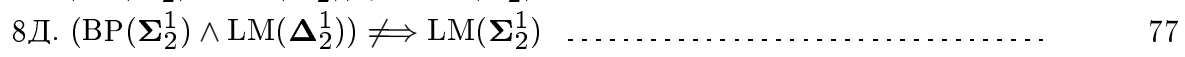

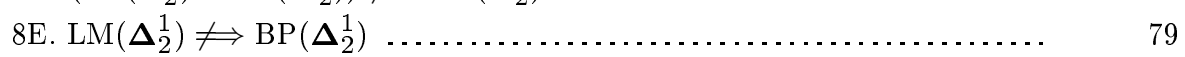

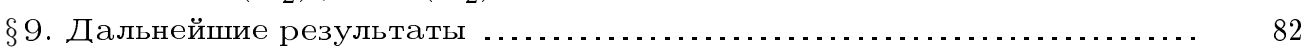

9А. Нужен ли недостижимьй кардинал? . . . . . . . . . . . . . . . . . . . . . 82

9Б. Проблемы третьего и четвертого проективных уровней $\quad . . \ldots \ldots \ldots . . . .28$

9В. Некоторые новые $\sigma$-УСА-идеалы и другие свойства регулярности . ... 83

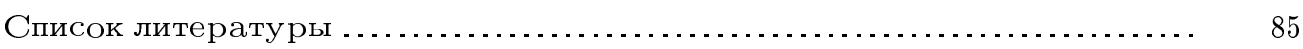

\section{Введение}

В августе 2001 года в Москве состоялась Международная математическая конференция, посвяшенная 100-летию со дня рождения П. С.Новикова, среди участников которой были и авторы настояшей статьи. Начало XXI века также знаменует 100-

Первьй автор поддержан РФФИ (грант № 03-01-00757), второй автор поддержан Минпромнауки РФ (грант № 37-053-11-0061). 
летний юбилей дескриптивной теории множеств и функций ${ }^{1}$, дисциплины, в которой П. С. Новикову принадлежат основополагающие результаты.

Одно из направлений деятельности П. С. Новикова в дескриптивной теории множеств - исследование свойств регулярности, т.е. свойств совершенного ядра, измеримости по Лебегу и свойства Бэра. Условимся обозначать через К какой-то класс точечных множеств. (Под точечнылми пока понимаются множества чисел вешественной прямой $\mathbb{R}$ или пространства $\mathbb{R}^{n}$, как это, в обшем, и было на раннем, “лузинском" этапе развития дескриптивной теории множеств. Современное, несколько более широкое понимание точечных множеств приводится в п. 1А.) Выражение "K-мно жество" означает: множество из класса К. Главньй вопрос формулируется здесь так:

для фиксированного класса $\mathbf{K}$ и одного из трех вьишеуказанных свойств регулярности верно ли, что каждое $\mathbf{K}$-множество обладает этим свойством?

Для дальнейшего обсуждения явно сформулируем три возможных ответа, три гипотезы о главных свойствах регулярности ${ }^{2}$ :

$\mathrm{PK}(\mathbf{K})$ : всякое $\mathbf{K}$-множество $X$ имеет свойство совершенного ядра, т.е. $X$ - конечное или счетное, или содержит совершенное подмножество (т.е. непустое, замкнутое и не имеюшее изолированных точек);

$\mathrm{LM}(\mathbf{K})$ : всякое $\mathbf{K}$-множество $X$ измеримо по Лебегу;

$\mathrm{BP}(\mathbf{K})$ : всякое $\mathbf{K}$-множество $X$ имеет свойство Бәра, т.е. $X$ отличается от какого-то борелевского множества на тощее ${ }^{3}$ множество.

Более обшие формулировки, применимые не только для множеств в $\mathbb{R}$, но и для множеств в любом несчетном польском ${ }^{4}$ пространстве, даны ниже в п. 1Б.

Свойство совершенного ядра возникло как один из подходов к проблеме мошности точечных множеств: действительно, совершенное подмножество вещественной прямой имеет мошность континуума $\mathfrak{c}=2^{\aleph_{0}}$. Измеримость (и свойство Бэра) относятся к основным понятиям математики. Примеры нерегулярных, например неизмеримых точечных множеств (Витали, Бернштейн и др.) были известны с начала ХХ века, однако все они получаются при помоши аксиомы выбора, т.е. не являются индивидуальными, недвусмысленно определенньми множествами. Желание получить более явные, “эффективные” контрпримеры вызвало повьшенньй интерес к "явно определенным" точечным множествам, изучавшимся в дескриптивной теории множеств. К середине 20-х годов к таким множествам относили борелевские множества (они образуют наименьшую $\sigma$-алгебру, содержашую все открытые множества данного пространства), $A$-множества (замыкание борелевской $\sigma$-алгебры относительно А-операции) и проективные множества (замыкание борелевской $\sigma$-алгебры относительно операций непрерьвного образа и дополнения). Последние организованы в иерархию классов

\footnotetext{
${ }^{1}$ Истоки дескриптивной теории можно связать либо с открытием борелевских множеств и бэровских функций в работах Бореля [13] (1898 г.) и Бэра [7] (1899 г.), либо с мемуаром Лебега [47] (1905 г.), систематизировавшим самые первые результаты о таких множествах и о функциях.

${ }^{2}$ Расшифровка аббревиатур: perfect kernel, Lebesgue measurability, Baire property. Эти три свойства известны как главные свойства регулярности. Рассматриваются и другие свойства этого типа; о некоторых из них будет сказано в п. 9В.

${ }^{3}$ тощим (meager) или мно жеств ом 1-й категор ии называется счетное объединение нигде не плотных множеств. Множество, дополнительное к тощему, назьвается котощим.

${ }^{4}$ Польскими называются сепарабельные полные метрические пространства.
} 
$\boldsymbol{\Sigma}_{n}^{1}, \boldsymbol{\Pi}_{n}^{1}, \boldsymbol{\Delta}_{n}^{1}$ множеств (или классов $\mathrm{A}_{n}, \mathrm{CA}_{n}, \mathrm{~B}_{n}$ в классической системе обозначений), возрастаюших с ростом индекса $n$, причем класс $\Pi_{n}^{1}$ состоит из дополнений $\boldsymbol{\Sigma}_{n}^{1}$-множеств, а класс $\boldsymbol{\Delta}_{n}^{1}$ - это пересечение классов $\boldsymbol{\Sigma}_{n}^{1}$ и $\boldsymbol{\Pi}_{n}^{1}$ (точное определение см. в п. 1A).

Благодаря $\sigma$-аддитивности меры и категории, все борелевские множества на прямой, разумеется, измеримы по Лебегу и имеют свойство Бэра. Вопрос о свойстве совершенного ядра для борелевских множеств не столь прост: прямая индукция по построению множеств с помощью борелевских операций не проходит. Решение было найдено П. С. Александровым [3] и Ф. Хаусдорфом [23] независимо и разными методами. Доказательство, данное Александровым, привело к открытию A-операции и $A$-множеств (современное название последних: $\Sigma_{1}^{1}$-множества) М. Я. Суслиным [91]. Н.Н. Лузин [51] и Суслин установили, что А-множества имеют все три свойства регулярности, т.е. в наших обозначениях для них выполняется $\operatorname{PK}\left(\boldsymbol{\Sigma}_{1}^{1}\right), \operatorname{LM}\left(\boldsymbol{\Sigma}_{1}^{1}\right), \operatorname{BP}\left(\boldsymbol{\Sigma}_{1}^{1}\right)$. Конечно, измеримость и свойство Бэра переносятся и на класс $\boldsymbol{\Pi}_{1}^{1}$ дополнительных множеств (классически известных как $C A$-множества).

Все предпринятые в классической дескриптивной теории попытки распространить эти результаты на более высокие проективные классы, т.е. доказать или опровергнуть хотя бы $\operatorname{PK}\left(\boldsymbol{\Pi}_{1}^{1}\right), \operatorname{LM}\left(\boldsymbol{\Sigma}_{2}^{1}\right), \operatorname{BP}\left(\boldsymbol{\Sigma}_{2}^{1}\right)$, оказались неудачньмми. Очень быстро эти проблемы были осознаны как трудные и, вероятно, центральные для дескриптивной теории. Более того, Лузин высказьвает в [53] (1925) убеждение, что эти проблемы вообше неразрешимы, т.е. на них невозможно дать определенный ответ, а в [52] обсуждает неразрешимость и более общих предложений о свойстве совершенного ядра, измеримости и свойстве Бэра для всех проективных множеств. ${ }^{5}$ Позже П. С. Новиков охарактеризовал проблемы о совершенном ядре для $\Pi_{1}^{1}$-множеств и измеримости для $\Sigma_{2}^{1}$-множеств в [78] (1951) как две из трех “основных проблем дескриптивной теории функций". 6

Итак, развитие классической дескриптивной теории множеств привело к проблемам о наличии свойств регулярности у точечных множеств как в их "минимальной" (с точки зрения участвуюшего в них проективного класса $)^{7}$ и наиболее непосредственной форме, т.е.

$$
\operatorname{PK}\left(\boldsymbol{\Pi}_{1}^{1}\right), \quad \operatorname{LM}\left(\boldsymbol{\Delta}_{2}^{1}\right), \quad \operatorname{BP}\left(\boldsymbol{\Delta}_{2}^{1}\right), \quad \operatorname{LM}\left(\boldsymbol{\Sigma}_{2}^{1}\right), \quad \operatorname{BP}\left(\boldsymbol{\Sigma}_{2}^{1}\right),
$$

так и в целом для класса всех проективных множеств ${ }^{8}$. Отсутствие каких-либо разум-

\footnotetext{
5 “Неизвестно и никогда не будет известно, имеет ли каждое несчетное множество данного семейства [т.е. семейства проективных множеств] мощность континуума, является оно или нет мно жеств ом третьей категории [т.е. множеством, не имеющим свойства Бэра], измеримо ли оно. Это было написано в [52] за десятки лет до создани методов, которые позволили действительно доказать неразрешимость этих проблем. И более того, когда господствовало представление наоборот о разрешимости любой математической проблемы.

${ }^{6}$ Третьей в списке Новикова стоит проблема отделимости в высших проективных классах, ставшая особенно интересной после его собственной работы [76], где показано, что законы отделимости для второго проективного уровня обратны законам отделимости для первого уровня (см. комментарии Лузина в [58; $\S 23]$ ). Сюда можно добавить проблему униформизации, впервые рассмотренную в контексте дескриптивной теории множеств в [56], и проблемы о несчетных последовательностях борелевских множеств (см., например, у Лузина в [58; § 23] или [59]). Об этих проблемах, которые мы здесь не обсуждаем, говорится в обзорах и статьях [36], [38], [40], [93], $[97]$.

${ }^{7}$ Разумеется, не считая доказуемых утверждений для класса $\boldsymbol{\Sigma}_{1}^{1}$.

${ }^{8}$ Строго говоря, $\boldsymbol{\Sigma}_{2}^{1}$-случай проблем измеримости и свойства Бэра не минимален, посколшку
} 
ных подходов к их решению, в одну сторону или в другую, быстро привело к мысли о неразрешимости этих проблем.

Скажем несколько слов о самом понятии неразрешимости.

Вопросынеразрешимости, т.е. невозможности вьполнить построение определенного математического объекта (в том числе, построить доказательство или опровержение какой-то гипотезы) определенными средствами, были известны в математике задолго до возникновения дескриптивной теории множеств: упомянем теорему Абеля о неразрешимости в радикалах алгебраических уравнений высших степеней или античные проблемы о трисекции угла, квадратуре круга и удвоении куба. Однако строгая постановка задачи о неразрешимости теоретико-множественных проблем возникла после создания аксиоматической теории множеств ZFC Цермело-Френкеля. Эта аксиоматическая система была разработана (1908-1925), в частности, в связи с установкой сделать математические доказательства более аккуратными и кодифицировать все используемые в них аксиомы. Интересно, что, в общем, не было аксиом, о которых велись бы споры, включать их или нет в эту аксиоматическую теорию множеств. ${ }^{9}$ После длительной проработки оснований всех разделов математики считается твердо установленньм, что любое математическое рассуждение может быть преобразовано в доказательство из аксиом ZFC (или, как говорят, - в вьввод в теории ZFC). И в этом смысле недоказуемость какого-то положения $\boldsymbol{P}$ в $\mathbf{Z F C}$ означает недоказуемость $\boldsymbol{P}$ в математике. Итак, неразрешимость какой-либо математической проблемы, т.е. невозможность дать ни положительный, ни отрицательный ответ на поставленньй вопрос, приравнивается к ее неразрешимости в теории ZFC. Последняя же означает, что формула $\boldsymbol{P}$, выражающая данную проблему, не имеет вьвода в теории $\mathbf{Z F C}$, как и ее отрицание $\neg \boldsymbol{P}$.

Вьводимость какой-либо формулы $\boldsymbol{P}$ в ZFC равносильна по теореме Гёделя о полноте тому, что $\boldsymbol{P}$ выполняется во всех моделях теории ZFC. Поэтому типичное доказательство неразрешимости какой-то проблемы $\boldsymbol{P}$ в Z ZFC состоит в том, что строится какая-то модель $M$ (для всех) аксиом $\mathbf{Z F C , ~ в ~ к о т о р о и ̆ ~} \boldsymbol{P}$ истинна (т.е. $\boldsymbol{P}$ имеет в $M$ положительное решение), и другая модель $N$ аксиом ZFC, в которой $\boldsymbol{P}$ ложна (т.е. $\boldsymbol{P}$ решается в $N$ отрицательно). Первая часть этой задачи доказьвает непротиворечивость присоединения $\boldsymbol{P}$ к аксиомам ZFC, а вторая - непротиворечивость присоединени $\neg \boldsymbol{P}$ (к аксиомам ZFC). ${ }^{10}$ Конечно, бьвают доказательства, в которых одна или обе эти части (т.е. о $\boldsymbol{P}$ или о $\neg \boldsymbol{P}$ ) получаются редукцией к другим уже известньм

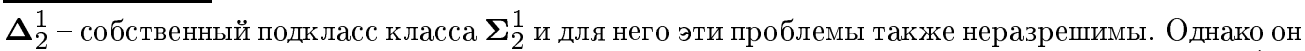
заслуживают включения в список, по крайней мере, по двум причинам. Во-первых, класс $\boldsymbol{\Sigma}_{2}^{1}=$ $\mathrm{A}_{2}$ вообше привлекал больше внимания в классической дескриптивной теории, чем класс $\Delta_{2}^{1}=$ $\mathrm{B}_{2}$. Во-вторых, методы решения этих проблем для $\boldsymbol{\Sigma}_{2}^{1}$ и $\boldsymbol{\Delta}_{2}^{1}$-случаев, в сущности, одинаковы.

${ }^{9}$ Если не считать дискуссии по поводу аксиомы выбора, которая велась скорее в рамках полемики о допустимости таких средств, как закон исключенного третьего, неэффективные построения, "актуально" бесконечные множества и т. п., в которой отрицание аксиомы выбора скорее означало отрицание вообще любой аксиоматики. Этой теме, в значительной мере, посвящень знаменитые "Cinq lettres" [8] - переписка между Адамаром, Бэром, Борелем и Лебегом. Лузин говорит об этом в [58; ч. III], [55; пп. $31,60,64]$ или в [57], главе 1 и заключении.

${ }^{10} \mathrm{~B}$ се рассуждения о неразрешимости в $\mathbf{Z F C}$ и непротиворечивости относительно ZFC, конечно, предполагают, что сама эта теория $\mathbf{Z F C}$ непротиворечива, т.е. в ней невозможно вьвести некоторую формулу $\Phi$ и одновременно вьвести $\neg \Phi$ (что эквивалентно невозможности вывести в ней любую формулу). Доказать непротиворечивость ZF C средствами современной математики невозможно, так как это означало бы доказательство непротиворечивости теории $\mathbf{Z F C}$ из ее собственных аксиом, что исключается второй теоремой Гёделя о неполноте. Тем не менее, сво- 
результатам о непротиворечивости.

Первые результаты в направлении доказательства непротиворечивости и неразрешимости теоретико-множественных предложений были получены К. Гёделем [20] (1938-1940). Построенная им структура - класс L всех конструктивных множеств с обычньм отношением $\in$ принадлежности двух множеств - является наименьшей моделью аксиом ZFC, содержащей все ординалы (порядковые числа). Гёдель доказал, что в $\mathbf{L}$ верна обобщенная континуум-гипотеза $\mathbf{G C H}$ и, тем самьм, установил ее непротиворечивость. Исследования класса $\mathbf{L}$ были продолжены в работе П. С. Новикова [78] (1951), где им доказано, что проблемы $\operatorname{PK}\left(\boldsymbol{\Pi}_{1}^{1}\right)$ и $\operatorname{LM}\left(\boldsymbol{\Delta}_{2}^{1}\right)$ (а тем самым, и $\operatorname{LM}\left(\boldsymbol{\Sigma}_{2}^{1}\right)$, поскольку $\boldsymbol{\Delta}_{2}^{1}-$ часть класса $\boldsymbol{\Sigma}_{2}^{1}$, а также, в силу совершенно аналогичных соображений, $\mathrm{BP}\left(\boldsymbol{\Delta}_{2}^{1}\right)$ и $\left.\mathrm{BP}\left(\boldsymbol{\Sigma}_{2}^{1}\right)\right)$ решаются в $\mathbf{L}$ отрицательно, т.е. там имеются соответствующие явно определенные контрпримеры. Тем самьм, была установлена непротиворечивость отрицательных решений проблем регулярности для указанных проективных классов.

Второй, в определенном смысле противоположньй метод построени моделей ZFC - это открытьй П.Коэном (1963) метод вынуждения (или форсинг): общий метод расширения любой исходной модели $M$ теории $\mathbf{Z F C}$ (например, класса $\mathbf{L}$ ) посредством присоединения некоторого множества $a \notin M$ (и, конечно, всех "производных" множеств, т.е. таких, что их сушествование в модели $M[a]$ вытекает из существования в ней $a$ ) таким образом, что расширенная структура $M[a]$ снова удовлетворяет всем аксиомам ZFC. Сам Коэн использовал этот метод для построения модели, в которой GCH (даже СН) нарушаетс (1962-1963, см. книгу [15]). Несколько позже Р. Соловей [89] (1970) построил модель ZFC, в которой каждое проективное множество удовлетворяет всем трем свойствам регулярности. Вместе с указанньми вьше результатами П. С. Новикова этот результат привел к тому, что проблемы регулярности точечных множеств оказались неразрешимьми. Причем как в "минимальных" случаях $\operatorname{PK}\left(\boldsymbol{\Pi}_{1}^{1}\right), \operatorname{LM}\left(\boldsymbol{\Delta}_{2}^{1}\right), \operatorname{BP}\left(\boldsymbol{\Delta}_{2}^{1}\right), \operatorname{LM}\left(\boldsymbol{\Sigma}_{2}^{1}\right), \operatorname{BP}\left(\boldsymbol{\Sigma}_{2}^{1}\right)$, так и в целом для класса всех проективных множеств, т.е. лузинская гипотеза о неразрешимости этих пяти проблем была подтверждена. Заметим, что, в отличие от гёделевских теорем о неполноте или, скажем, результата о континуум-гипотезе, речь здесь идет о неразрешимости конкретных математически значимых свойств вполне индивидуально определенных и достаточно простых точечных множеств. Например, имеется вполне определенное $\Pi_{1}^{1}$-множество $X \subseteq \mathbb{R}$ такое, что утверждение $\mathrm{PK}(X)$ о том, что $X$ имеет свойство совершенного ядра, неразрешимо.

Кроме теорем о неразрешимости исследования разных авторов на рубеже $1960-\mathrm{x}-$ 70-х годов позволили установить удивительные связи между рассматриваемыми проблемами. Оказалось, что $\operatorname{PK}\left(\boldsymbol{\Pi}_{1}^{1}\right)$ влечет $\operatorname{LM}\left(\boldsymbol{\Sigma}_{2}^{1}\right)$ и $\mathrm{BP}\left(\boldsymbol{\Sigma}_{2}^{1}\right)$, а несколько позже выяснилось, что $\operatorname{LM}\left(\boldsymbol{\Sigma}_{2}^{1}\right)$ влечет $\operatorname{BP}\left(\boldsymbol{\Sigma}_{2}^{1}\right)$. Эти результаты изображены на диаграмме. $\mathrm{K}$ концу 80-х годов с помошю метода вынуждения были построены модели, которые установили полноту этой диаграммы в том смысле, что никакие другие импликации между ее элементами не выводимы в ZFC. Эти работы, в целом, закончили цикл наиболее важных исследований по проблемам регулярности для проективных классов $\Pi_{1}^{1}$ (свойство совершенного ядра), $\boldsymbol{\Sigma}_{2}^{1}$ и $\boldsymbol{\Delta}_{2}^{1}$ (измеримость и свойство Бэра), как и в целом для всех проективных множеств. ${ }^{11}$

бодное от противоречий многолетнее развитие математики, явно или неявно на основе именно аксиом ZFC, делает гипотезу о ее непротиворечивости общепринятой.

${ }^{11}$ Мы не касаемся здесь тех исследований в рамках основанной Колмогоровым и Хаусдорфом 


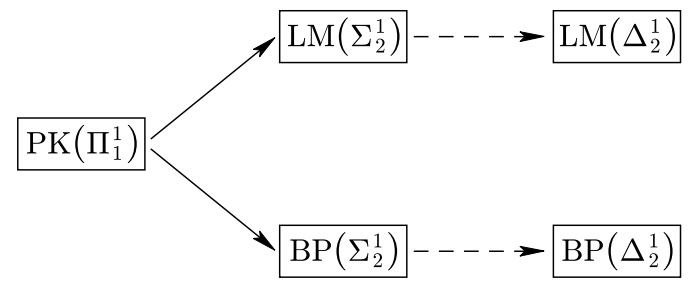

ДиАГрамма. Все пять гипотез из списка $(*)$ выше неразрешимы в ZFC; стрелки изображают доказуемые импликации между этими гипотезами; пунктирные стрелки изображают тривиальные (поскольку $\Delta_{2}^{1} \subseteq \Sigma_{2}^{1}$ ) среди них;

“производная" импликация $\mathrm{PK}\left(\boldsymbol{\Pi}_{1}^{1}\right) \Longrightarrow \mathrm{BP}\left(\boldsymbol{\Sigma}_{2}^{1}\right)$ выделена в силу того, что она была доказана вместе с $\operatorname{PK}\left(\boldsymbol{\Pi}_{1}^{1}\right) \Longrightarrow \operatorname{LM}\left(\boldsymbol{\Sigma}_{2}^{1}\right)$ и много раньше, чем $\operatorname{LM}\left(\boldsymbol{\Sigma}_{2}^{1}\right) \Longrightarrow \operatorname{BP}\left(\boldsymbol{\Sigma}_{2}^{1}\right)$

Цель этой статьи состоит в изложении всех основных результатов, полученных в этих исследованиях, с достаточно полными (и в то же время существенно упрошенными и модернизированными по сравнению с оригинальными работами) доказательствами, а также - систематизированное изложение соответствуюших методов. Статья рассчитана на читателя, интересуюшегося вопросами теории множеств и имеющего некоторьй опыт в этой области и в области математической логики и, по крайней мере для некоторых разделов статьи, хотя бы минимальное знакомство с форсингом. Что касается технической стороны (доказательств), то мы старались сделать изложение самодостаточньм, хотя, конечно, статья не может служить учебником по теориям конструктивности Гёделя и форсинга.

Теперь о структуре статьи. Параграф 1 носит характер общего введения. Параграфф 2 содержит введение в теорию конструктивных по Гёделю множеств (в тех рамках, которые необходимы для статьи). В частности, рассматривается определимость конструктивного построения внутри континуума.

Параграфы 3-5 посвяшены вьводу нетривиальных импликаций нашей диаграммы. Для этого с каждым из пяти представленных на диаграмме утверждений, т.е. с $\operatorname{PK}\left(\boldsymbol{\Pi}_{1}^{1}\right), \operatorname{LM}\left(\boldsymbol{\Sigma}_{2}^{1}\right), \operatorname{BP}\left(\boldsymbol{\Sigma}_{2}^{1}\right), \operatorname{LM}\left(\boldsymbol{\Delta}_{2}^{1}\right)$ и $\mathrm{BP}\left(\boldsymbol{\Delta}_{2}^{1}\right)$, ассоциируется резольвента - лузинский термин, обозначаюший такую (эквивалентную) переформулировку исходного предложения, которая имеет значительно более простую природу. Следствие 3.4 содержит все пять таких переформулировок - резольвент, каждая из которых представляет собой, естественно, эквивалентность. Более “эффективные” варианты этих эквивалентностей даются в теореме 3.3. Доказательства этих эквивалентностей в одну сторону приводится в $\S 3$ на основе теории конструктивности, а в другую сторону - в $\oint 4$ на основе метода вьнуждения (форсинга). Наконец, в $\S 5$ проблемы измеримости и свойства Бэра связьваются со свойствами финального доминирования (одного естественного отношения порядка на множестве $\mathbb{N}^{\omega}$ всех функций из натуральных чисел в себя), что позволяет вьвести импликацию $\operatorname{LM}\left(\Sigma_{2}^{1}\right) \Longrightarrow \operatorname{BP}\left(\Sigma_{2}^{1}\right)$. Этим завершается вьвод всех импликаций диаграммы.

Особенность стандартных доказательств нетривиальных импликаций из этой диаграммы состоит в том, что для получения достаточно просто формулируемых утверждений о природе точечных множеств низких проективных классов привлекаются методы (такие, как конструктивность и форсинг), которые существенно используют класс

теории операций над множествами, в которой были найдены внутри класса $\Delta_{2}^{1}$ болшшие классы точечных множеств, все еще сохраняющие измеримость и свойство Бэра. См. об этом в [39]. 
всех множеств. В $\S 6$ обсуждается, на наш взгляд, важньй вопрос: можно ли доказать все те же утверждения, не используя произвольных (так сказать, "канторовских") множеств и оставаясь в рамках тех понятий, которые участвуют в постановках самих проблем дескриптивной теории, т.е. используя только объекты типа точечных множеств, участвующих в этих постановках. На примере импликации $\mathrm{PK}\left(\boldsymbol{\Pi}_{1}^{1}\right) \Longrightarrow$ $\operatorname{LM}\left(\boldsymbol{\Sigma}_{2}^{1}\right)$ мы даем положительный ответ на этот вопрос и назьваем такие доказательства “элементарньми”, в противоположность доказательствам, использующим произвольные "канторовские" множества.

Следующий $\S 7$ содержит замечательньй результат: даже если предположить, что “нерегулярные" проективные множества существуют, например существует несчетное проективное множество без совершенного ядра (или неизмеримое, или не имеющее свойства Бэра), то отсюда не следует существование явно определимого "нерегулярного" проективного множества, т.е. одного конкретного, недвусмысленно определенного контрпримера! Различию между определимыми и "произвольными" (например, возникаюшими с помощью аксиомы выбора) множествами придавалось большое значение на ранних этапах развити теории множеств (см. сноску 9). Затем в $\S 7$ доказывается теорема Соловея: положительные решения всех трех проблем регулярности сразу для всех проективных множеств (и даже для некоторого еще более широкого класса множеств) не противоречат аксиомам ZFC.

В $\S 8$ устанавливается полнота приведенной вьше диаграммы. В частности, посредством построения подходящих моделей доказывается, что ни одна из ее импликаций не может быть обращена.

Заключительньй $\S 9$ содержит обзор тех результатов о свойствах регулярности, которые просто не поместились вместе с их доказательствами в одну статью. Здесь рассматривается роль недостижимого кардинала в некоторых доказательствах непротиворечивости, свойства регулярности для третьего проективного уровня и некоторые подобные вопросы.

\section{§. Борелевские и проективные множества}

Этот параграф носит вводньй характер. Мы даем определения проективной иерархии множеств из бэровских пространств и более тонкой эффективной иерархии, построенной на определимости точечных множеств аналитическими формулами, рассматриваем операцию решета и доказываем независимость проблем о свойствах регулярности от выбора базового пространства в категории совершенных польских пространств, а также независимость проблемы измеримости от выбора меры в категории борелевских нулевых в точках мер. Раздел заканчивается изложением кодировки как борелевских множеств, так и и счетных ординалов, которая играет важную роль в доказательствах и в понимании существа теорем.

1А. Вещественная прямая, польские пространства и бэровское пространство. Выйдя в начале XX века из теории функций, дескриптивная теори множеств долгое время развивалась, в основном, как теори точечных множеств вещественной прямой $\mathbb{R}$ и пространств $\mathbb{R}^{n}$. $\mathrm{K}$ середине 20 -х годов выяснилось, что основные результаты в этой области могут быть обобщены на все несчетные польские пространства. (Польским назьвается полное сепарабельное метрическое пространство.) Систематическое изложение для польских пространств обшего вида было впервые дано Хаусдорфом [25]. Несколько позже Куратовский [45] (см. также книгу 
[46; §37.II]) доказал следующую теорему, которая делает дескриптивную теорию множеств вообще мало зависящей от выбора несчетного польского пространства.

Теорема 1.1 (Куратовский). Любые два несчетных польских пространства $\mathbb{X}, \mathbb{Y}$ борелевски изоморфны, т.е. найдется (назьваемая борелевским изоморфизмом) биекиия $F: \mathbb{X} \stackrel{\text { на }}{\longrightarrow} \mathbb{Y}$ такая, что $F$-образы и F-прообразы борелевских множеств - снова борелевские множества.

Таким образом, по крайней мере в исследованиях тех вопросов дескриптивной теории, которые инвариантны (прямо или с соответствуюшими поправками) относительно борелевских изоморфизмов, можно ограничиться рассмотрением множеств в каком-нибудь одном, фиксированном несчетном польском пространстве. В качестве такового в современных работах обычно используется пространство Бэра $\mathbb{N} \omega .12$ Этой практике следует и наша статья. О том, какую форму принимают проблемы о свойствах регулярности в пространстве $\mathbb{N}^{\omega}$ и в произвольном несчетном польском пространстве, будет сказано в следующем пункте.

Перейдем к техническим деталям.

Через $2^{<\omega} \varsubsetneqq \mathbb{N}<\omega$ обозначаются множества всех конечных последовательностей чисел 0,1 и, соответственно, конечных последовательностей всех натуральных чисел. Пусть lh $s-$ длина $s \in \mathbb{N}<\omega$. Если $s \in \mathbb{N}^{<\omega}$ и $n \in \mathbb{N}$, то $s^{\wedge} n \in \mathbb{N}<\omega$ получается присоединением $n$ к $s$ в качестве самого правого члена. Запись $s \subset t$ означает, что последовательность $t$ - собственное продолжение последовательности $s$.

Пространство Бәра $\mathbb{N}^{\omega}$ (также обозначается через $\mathscr{N}$ ) образовано всеми бесконечньми последовательностями натуральных чисел. Его топология порождена 6 эровскими интервалами, т.е. множествами вида $\mathscr{N}_{s}=\left\{x \in \mathbb{N}^{\omega}: s \subset x\right\}, s \in \mathbb{N}<\omega$. Заметим, что пространства $\mathscr{N}^{\omega}$ и $\mathscr{N}^{(k, \ell)}=\mathscr{N}^{k} \times \mathbb{N}^{\ell}(k \geqslant 1)$ гомеоморфны $\mathbb{N}^{\omega}$ : например, гомеоморфизм $\mathbb{N} \omega \stackrel{\text { на }}{\longrightarrow} \mathscr{N}^{\omega}$ дается отображением $x \mapsto\left\{(x)_{n}\right\}_{n \in \mathbb{N}}$, где для $x \in \mathbb{N} \omega$ и $n \in \mathbb{N}$ точка $(x)_{n} \in \mathbb{N}^{\omega}$ определяется равенством $(x)_{n}(k)=x\left(2^{n}(2 k+1)-1\right), \forall k$.

Замкнутые множества пространства $\mathbb{N}^{\omega}$ допускают следующее полезное представление. Непустое множество $T \subseteq \mathbb{N}<\omega$ называется деревом, если мы имеем $t \in T$ всякий раз, когда $s \in T, t \in \mathbb{N}<\omega, t \subset s$ (отсюда следует, что пустая последовательность $\Lambda$ принадлежит $T)$. Множества вида $[T]=\left\{x \in \mathbb{N}^{\omega}: \forall m(x\lceil m \in T)\}\right.$, где $T \subseteq \mathbb{N}<\omega$ является деревом, - это в точности все непустые замкнутые подмножества $\mathbb{N}^{\omega}$, причем достаточно использовать лишь те деревья $T$, которые не имеют $\subseteq$-максимальных элементов.

Точкой ветвления дерева $T$ называется $s \in T$ такое, что $s^{\wedge} j \in T$ по крайней мере для двух разных $j \in \mathbb{N}$. Дерево $T$ называется совершенным, когда для каждого $t \in T$ найдется точка ветвления $s \in T$ с $t \subset s$. Множества вида $[T]$, где $T \subseteq \mathbb{N}<\omega-$ совершенное дерево, это все совершенные подмножества $\mathbb{N}^{\omega}$.

\footnotetext{
${ }^{12}$ Причиной такого предпочтения пространства $\mathbb{N}^{\omega}$ (в частности, перед вещественной прямой $\mathbb{R}$ ) являются некоторые топологические свойства $\mathbb{N}^{\omega}$, в частности, его нульмерность (см. комментарий 2 в [60; с. 725]), но главным образом то обстоятельство, что структура $\mathbb{N}^{\omega}$ допускает использование простого язька аналитических формул для описания точечных множеств и их свойств (см. п. 1В), что позволяет чрезвычайно упростить техническую сторону изложения. В то же время, пространство $\mathbb{N}^{\omega}$ мало уступает вещественной прямой в плане геометрической наглядности, поскольку оно изоморфно "бэровской прямой", т.е. множеству всех иррациональных чисел $\mathbb{R}$, через известное соответствие $x \mapsto \frac{1 \mid}{\mid x(0)+1}+\frac{1 \mid}{\mid x(1)+1}+\frac{1 \mid}{\mid x(2)+1}+\cdots$, $x \in \mathbb{N}^{\omega}$, использующее цепные дроби, или через более прямое построение, указанное в [4; с. 155$]$. "Бэровская прямая" рассматривается, например, в лузинских "Лекциях" [57].
} 
Канторов дисконтинуум $2^{\omega}$ можно рассматривать либо как замкнутое компактное подмножество $\mathbb{N} \omega$, либо как самостоятельное пространство, с топологией, порожденной канторовыми интервалами $\mathscr{C}_{s}=\left\{x \in 2^{\omega}: s \subset x\right\}, s \in 2^{<\omega}$.

Проективные классы $\boldsymbol{\Sigma}_{n}^{1}, \boldsymbol{\Pi}_{n}^{1}, \boldsymbol{\Delta}_{n}^{1}\left(\mathrm{~A}_{n}, \mathrm{CA}_{n}, \mathrm{~B}_{n}\right.$ в более ранней системе обозначений) для пространств вида $\mathscr{N}^{(k, \ell)}$ определяются индукцией по $n$ так:

$\Sigma_{0}^{1}$ состоит из всех открытых множеств пространств вида $\mathscr{N}^{(k, \ell)}$;

$\boldsymbol{\Pi}_{n}^{1}$ состоит из всех дополнений множеств класса $\boldsymbol{\Sigma}_{n}^{1}$;

$\boldsymbol{\Sigma}_{n+1}^{1}$ состоит из всех проекций множеств класса $\boldsymbol{\Pi}_{n}^{1}$, где проекиия множества $P \subseteq$ $\mathscr{N}^{(k+1, \ell)}$ есть множество $\left\{\langle\vec{x}, \vec{k}\rangle: \exists y \in \mathbb{N}^{\omega}\left(\left\langle\vec{x}^{\wedge} y, \vec{k}\right\rangle \in P\right)\right\} ;$

$\boldsymbol{\Delta}_{n}^{1}$ состоит из всех множеств, принадлежаших одновременно $\boldsymbol{\Sigma}_{n}^{1}$ и $\boldsymbol{\Pi}_{n}^{1}$;

$\boldsymbol{\Sigma}_{\infty}^{1}$ обозначает класс $\bigcup_{n} \boldsymbol{\Sigma}_{n}^{1}=\bigcup_{n} \boldsymbol{\Pi}_{n}^{1}$ всех проективных множеств.

Эквивалентным образом, можно определить $\boldsymbol{\Sigma}_{n+1}^{1}$ как совокупность всех непрерывных образов $\Pi_{n}^{1}$-множеств, расположенных в том же самом пространстве $\mathscr{N}^{(k, \ell)}$. В этой форме определение проективных классов распространяется на любое польское пространство $\mathbb{X}$ со следующим уточнением начального шага индукции: класс $\boldsymbol{\Sigma}_{1}^{1}$ по определению состоит из всех непрерьвных образов всех $\mathbf{G}_{\delta}$-множеств этого пространства. (Множеств класса $\Pi_{0}^{1}$, т.е. замкнутых, как в определении для $\mathscr{N}^{(k, \ell)}$, в обшем случае не хватает, поскольку, скажем, для $\sigma$-компактных пространств, как $\mathbb{R}$, проекции и вообще непрерывные образы замкнутых множеств принадлежат $\mathbf{F}_{\sigma}-$ собственному подклассу класса $\boldsymbol{\Sigma}_{1}^{1}$.)

1Б. Основные свойства регулярности в польских пространствах. Сразу видно, что в то время, как проблемы совершенного ядра и свойства Бэра (см. введение) имеют очевидный и точньй смысл для любого топологического пространства, проблема измеримости связана еще и с выбором меры. Чтобы рассмотреть этот вопрос подробнее, уточним некоторые понятия, связанные с мерой.

Под борелевской мерой на пространстве $\mathbb{X}$ мы будем понимать любую $\sigma$-аддитивную и $\sigma$-конечную (т.е. $\mathbb{X}$ есть счетное объединение борелевских множеств конечной меры) меру $\mu$, заданную на $\sigma$-алгебре всех борелевских подмножеств $\mathbb{X}$ и удовлетворяюшую $\mu(\mathbb{X})>0$ и $\mu(\{x\})=0$ для каждой точки $x \in \mathbb{X} .{ }^{13}$

Мера $\mu$ назьвается конечной, если значение $\mu(\mathbb{X})$ конечно, и $\sigma$-конечной, если $\mathbb{X}-$ счетное объединение множеств конечной меры.

Лебеговское продолжение меры $\mu$ на $\mathbb{X}$, которое мы будем обозначать тем же символом $\mu$, определено на $\sigma$-алгебре всех множеств $X \subseteq \mathbb{X}$ таких, что найдутся борелевские множества $U, V$ с $U \subseteq X \subseteq V$ и $\mu(V \backslash U)=0$; такие множества $X$ называют $\mu$-измеримыми и полагают $\mu(X)=\mu(U)=\mu(V)$.

Поскольку проективные классы замкнуты относительно пересечений с борелевскими множествами, измеримость проективных множеств относительно $\sigma$-конечных борелевских мер следует из измеримости относительно конечных мер. С учетом этого замечания, наши гипотезы PK, LM, BP (см. введение) можно сфформулировать для данного польского пространства $\mathbb{X}$ и данного проективного класса $\mathbf{K}$ так:

$\mathrm{PK}_{\mathbb{X}}(\mathbf{K})$ : всякое $\mathbf{K}$-множество $X \subseteq \mathbb{X}$ имеет свойство совершенного ядра;

\footnotetext{
${ }^{13}$ Последнее требование означает, что $\mu$ исчезает в точках - и это свойство молчаливо предполагается для борелевских мер в настоящей статье. Нетрудно проверить, что это требование не умаляет общности рассмотрения тех вопросов, о которых идет здесь речь, по крайней мере в категории $\sigma$-конечных мер.
} 
$\mathrm{LM}_{\mathbb{X}}^{\mu}(\mathbf{K}):$ всякое $\mathbf{K}$-множество $X \subseteq \mathbb{X}$ измеримо в смысле данной борелевской конечной меры $\mu$ на $\mathbb{X}$;

$\mathrm{BP}_{\mathbb{X}}(\mathbf{K})$ : всякое $\mathbf{K}$-множество $X \subseteq \mathbb{X}$ имеет свойство Бэра.

ЗАмЕчАниЕ 1.2. Если фиксирован проективный класс $\mathbf{K}$, то каждое из этих трех утверждений не зависит от выбора несчетного польского пространства $\mathbb{X}$ и, в отношении LM, от выбора меры $\mu$ в категории борелевских конечных мер. Для измеримости это прямо вытекает из теоремы 1.1 и нижеследующей леммы 1.3 , поскольку любой проективньй класс инвариантен относительно борелевских изоморфизмов. Для РК используем то, что всякое борелевское несчетное множество в польском пространстве содержит совершенное подмножество. Что же касается свойства Бэра, искомьй результат очевиден из другой теоремы: если $\mathbb{X}$ - совершенное польское пространство, то найдется множество $Y \subseteq \mathbb{X}$, гомеоморфное $\mathbb{N}^{\omega}$ и такое, что $\mathbb{X} \backslash Y$ - тошее множество в $\mathbb{X} .{ }^{14}$

Лемма 1.3. Пусть $\mu, \nu$ - борелевские конечные меры на польских пространствах $\mathbb{X}, \mathbb{Y}$, а $\mathbf{K}$ - проективньй класс. Тогда существуют борелевские множества $X \subseteq \mathbb{X}$ полной $\mu$-меры и $Y \subseteq \mathbb{Y}$ полной $\nu$-меры и борелевская биекиия $h: X \stackrel{\text { на }}{\longrightarrow} Y$, переводящая $\mu$ в $\nu$. Следовательно, $\operatorname{LM}_{\mathbb{X}}^{\mu}(\mathbf{K}) \Longleftrightarrow \operatorname{LM}_{\mathbb{Y}}^{\nu}(\mathbf{K})$.

ДокАЗАТЕльство. Мы можем предполагать, что $\mu(\mathbb{X})=\nu(\mathbb{Y})=1$, а также, в силу замечания 1.2, что $\mathbb{X}=\mathbb{Y}=[0,1]$ (отрезок вещественной прямой). Удалив из $[0,1]$ все интервалы $(a, b) \quad \mu$-меры 0 , мы имеем совершенное множество $X \subseteq[0,1]$ с $\mu(X)=1$ и $\mu(I \cap X)>0$ всякий раз, когда интервал $I=(a, b)$ непусто пересекается с $X$. Аналогично, найдется совершенное множество $Y \subseteq[0,1]$ с $\nu(Y)=1$ и $\nu(I \cap Y)>0$ всякий раз, когда интервал $I$ непусто пересекается с $Y$.

Положим $f(x)=\mu\left(X^{\prime} \cap[0, x)\right)$ для $x \in X$; легко видеть, что $f$-сохраняющая порядок непрерьвная функция из $X$ на $[0,1]$. Более того, $f$ переводит меру $\mu$ на $X$ в обычную лебегову меру на $[0,1]$. Наконец, $f$ "почти” взаимно однозначна в том смысле, что $f(x)=f(y)$ вьполнено при $x \neq y$ только в том случае, когда $x, y$-концы одного и того же смежного интервала к $X$, так что если мы удалим из $X$ все, например, левые концы таких интервалов, то $f$ будет биекщией на полученном множестве $X^{\prime}$, и все еше $\mu\left(X^{\prime}\right)=1$.

Построив таким же образом, но исходя из $\nu$, функцию $g: Y \stackrel{\text { на }}{\longrightarrow}[0,1]$ и множество $Y^{\prime} \subseteq Y$ с $\nu\left(Y^{\prime}\right)=1$, мы получаем борелевский изоморфизм $h(x)=g^{-1}(f(x)): X^{\prime} \stackrel{\text { на }}{\longrightarrow}$ $Y^{\prime}$, переводящий $\mu$ в $\nu$, откуда следует лемма, поскольку борелевские биекции сохраняют проективньй класс.

Таким образом, наши гипотезы можно, не умаляя общности, рассматривать только для множеств какого-нибудь одного несчетного польского пространства $\mathbb{X}$ и, в отношении измеримости, только для одной борелевской конечной меры на $\mathbb{X}$. В качестве $\mathbb{X}$ удобно взять пространство Бэра $\mathbb{N}^{\omega}$ для $\mathrm{PK}$ и ВР и канторов дисконтинуум $2^{\omega}$ для LM

\footnotetext{
${ }^{14}$ Для доказательства заметим, что если $\varnothing \neq U \subseteq \mathbb{X}$ открыто, то ко всякому $\varepsilon>0$ найдется счетное объединение попарно дизъюнктных непустых окрестностей $U^{\prime} \subseteq U$ диаметра $<\varepsilon$, плотное в $U$ и такое, что замькание каждой $U^{\prime}$ включено в $U$. Это позволяет построить систему окрестностей $\left\{U_{s}\right\}_{s \in \mathbb{N}<\omega}$ такую, что $U_{\Lambda}=\mathbb{X}$, диаметр $U_{s}$ не превосходит $(\operatorname{lh} s)^{-1}$ при $\operatorname{lh} s \geqslant 1$, замькание $U_{s} \wedge n$ включено в $U_{s}$ и $\bigcup_{n} U_{s} \wedge n$ плотное подмножество $U_{s}$ при любом $s$. Множество $Y=\bigcap_{n} \bigcup_{n=\operatorname{lh} s} U_{s}=\bigcup_{a \in \mathbb{N}^{\omega}} \bigcap_{n} U_{a \nmid n}$ гомеоморфно $\mathbb{N}^{\omega}$ через отображение, отсылающее каждую точку $a \in \mathbb{N}^{\omega}$ в единственную точку пересечения $\bigcap_{n} U_{a} \uparrow n$.
} 
с вероятностной мерой $\lambda$, сообщающей каждому канторову интервалу $\mathscr{C}_{s}, s \in 2^{<\omega}$, меру $\boldsymbol{\lambda}\left(\mathscr{C}_{s}\right)=2^{-\operatorname{lh} s} \cdot{ }^{15}$ Впрочем, меру $\boldsymbol{\lambda}$ можно формально продолжить на $\mathbb{N} \omega$ условием $\boldsymbol{\lambda}\left(\mathbb{N}^{\omega} \backslash 2^{\omega}\right)=0$, т.е. $\boldsymbol{\lambda}(X)=\boldsymbol{\lambda}\left(X \cap 2^{\omega}\right)$ для $X \subseteq \mathbb{N}^{\omega}$; тогда $\mathrm{LM}_{\mathbb{N} \omega}^{\boldsymbol{\lambda}}(\mathbf{K})$ равносильно $\mathrm{LM}_{2}^{\boldsymbol{\lambda}}(\mathbf{K})$ для любого проективного класса $\mathbf{K}$.

Эти рассуждения приводят наши гипотезы о совершенном ядре, измеримости и свойстве Бэра к следуюшей окончательной формулировке, которая равносильна первоначальной формулировке (для вешественной прямой) во введении.

$\mathrm{PK}(\mathbf{K})$ : всякое $\mathbf{K}$-множество $X \subseteq \mathbb{N}^{\omega}$ имеет свойство совершенного ядра;

$\operatorname{LM}(\mathbf{K}):$ всякое $\mathbf{K}$-множество $X \subseteq 2^{\omega}$ (или, что равносильно, всякое $\mathbf{K}$-множество $\left.X \subseteq \mathbb{N}^{\omega}\right) \boldsymbol{\lambda}$-измеримо;

$\mathrm{BP}(\mathbf{K})$ : всякое $\mathbf{K}$-множество $X \subseteq \mathbb{N}^{\omega}$ имеет свойство Бэра.

Следующая теорема суммирует некоторые классические результаты.

Tеорема 1.4. Виполнены $\operatorname{PK}\left(\Sigma_{1}^{1}\right), \operatorname{LM}\left(\Sigma_{1}^{1}\right), \operatorname{BP}\left(\Sigma_{1}^{1}\right)$, т.е. каждое $\boldsymbol{\Sigma}_{1}^{1}$-мно-

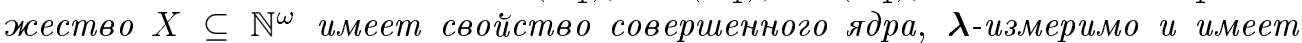
свойство Бэра. Результат для LM и $\mathrm{BP}$ в равной степени справедлив и для класса $\boldsymbol{\Pi}_{1}^{1}$.

ДоКАЗАТЕльство. Утверждения для измеримости и свойства Бэра будут ниже доказаны в более общем виде (теорема 3.7 ), поэтому сосредоточимся на РК. Известно, что $\boldsymbol{\Sigma}_{1}^{1}$-множества пространства $\mathbb{N}^{\omega}$ - это непрерьвные образы замкнутых подмножеств $\mathbb{N}^{\omega}$. Пусть $X=F^{\prime \prime} P$, где $P \subseteq \mathbb{N}^{\omega}$ замкнуто, а $F: P \rightarrow \mathbb{N}^{\omega}$-непрерьвная функщия. Назовем "хорошим" всякий бэровский интервал $U \subseteq \mathbb{N}^{\omega}$ такой, что $F$-образ $F "(U \cap P)$ несчетен. Так, само $\mathbb{N} \omega$ "хорошее", ибо $X$ несчетно, и если $U$ - "хорошее" множество, то найдутся "хорошие" множества $U_{1}, U_{2} \subseteq U$ диаметра менее половины диаметра $U$ и такие, что $F$ " $\left(U_{1} \cap P\right)$ не пересекается с $F$ " $\left(U_{2} \cap P\right)$, в частности, сами $U_{1}, U_{2}$ дизъюнктны. Это позволяет построить расшепляющуюся систему $\left\{U_{s}\right\}_{s \in 2^{<\omega}}$ "хороших" множеств $U_{s} \subseteq U$ такую, что:

(1) $U_{s} \wedge i \subseteq U_{s}$ и диаметр $U_{s} \wedge i$ не больше половины диаметра $U_{s}$;

(2) $F "\left(U_{s} \wedge_{0} \cap P\right)$ не пересекается с $F "\left(U_{s} \wedge_{1} \cap P\right)$.

Множество $C=\bigcap_{m} \bigcup_{m=1 h s} U_{s} \subseteq P$ совершенно и компактно, а $F$ взаимно однозначна на $C$, поэтому $Y=F " C$ - совершенное подмножество $X$.

1В. Проективная иерархия и язык аналитических формул. Структура пространства Бэра $\mathscr{N}=\mathbb{N}^{\omega}$ позволяет использовать простой язьк для описания множеств в пространствах $\mathscr{N}^{(k, \ell)}$. Этот язык использует два типа переменных, mun 0 с областью пробегания $\mathbb{N}$ (используются буквы $k, l, m, n$ и т.п.) и $m u n 1$ с областью пробегания $\mathbb{N}^{\omega}$ (используются буквы $x, y, z, a, b, c$ и т. п.). Разрешается образовывать тер.мы с применением подстановки терма или переменной типа 0 в переменную типа 1 и рекурсивных (т.е. вычислимых) функций из $\mathbb{N}^{\ell}$ в $\mathbb{N}$; например, $x\left(2^{k}+y(3 n)\right)$ является термом. Понятно, что термы суть объекты типа 0. Среди формул этого языка выделяются:

- әлементарные - формулы вида $t=t^{\prime}, t<t^{\prime}, t \leqslant t^{\prime}$, где $t, t^{\prime}$ - термы (например, переменные типа 0);

\footnotetext{
${ }^{15}$ Предпочтение, отданное $2^{\omega}$ перед $\mathbb{N}^{\omega}$, объясняется прежде всего компактностью $2^{\omega}$, играющей роль в некоторых выкладках, а также несуществованием такой борелевской меры на $\mathbb{N} \omega$, которая дает равную меру бэровским интервалам равного ранга. Отметим также, что отображение, сопоставляющее каждой точке $a \in 2^{\omega}$ вещественное число $r_{a}=\sum_{n=0}^{\infty} a(n) 2^{-n-1}$, переводит меру $\boldsymbol{\lambda}$ в обычную лебеговскую меру на $[0,1]$.
} 
- аналитические - все формулы, которые получаютс из элементарных при помоши логических связок и кванторов;

- арифметические - те аналитические формулы, которые не включают кванторов типа 1 (т.е. по $\mathbb{N}^{\omega}$ );

- ограниченные - те аналитические формулы, которые включают кванторы только вида $\exists k<t$ и $\forall k<t$, где $k$ - переменная типа 0 , а $t$ - терм; в частности, все бескванторные формулы - ограниченные;

- $\Sigma_{n}^{0}$ и $\Pi_{n}^{0}$ - арифметические формулы, соответственно, вида

$$
\exists k_{1} \forall k_{2} \exists k_{3} \ldots \exists(\forall) k_{n} \varphi \text { и } \forall k_{1} \exists k_{2} \forall k_{3} \ldots \forall(\exists) k_{n} \varphi
$$

где $\varphi$ - ограниченная формула;

- $\Sigma_{n}^{1}$ и $\Pi_{n}^{1}$ - аналитические формулы, соответственно, вида

$$
\exists x_{1} \forall x_{2} \exists x_{3} \ldots \exists(\forall) x_{n} \varphi \text { и } \forall x_{1} \exists x_{2} \forall x_{3} \ldots \forall(\exists) x_{n} \varphi,
$$

где $\varphi$ - арифметическая формула.

Эффективная иерархия. Свободные переменные аналитических формул можно заменять конкретными элементами $\mathbb{N}$ (тип 0) или $\mathbb{N}^{\omega}$ (тип 1), которые в этом случае называются параметрами. (Это существенно только для типа 1, так как любое натуральное число определимо бескванторной формулой.) Отсюда производится классификация множеств с точки зрения параметров, участвуюших в определении, а не только с точки зрения типа определяющей формулы.

Если задан “вектор" $\vec{a}=\left\langle a_{1}, \ldots, a_{j}\right\rangle \in\left(\mathbb{N}^{\omega}\right)^{j}$, то через $\Sigma_{n}^{i}(\vec{a})$ обозначается класс всех множеств в пространствах $\mathcal{N}^{(k, \ell)}$, которые определимы $\Sigma_{n}^{i}$-формулами с параметрами из списка $a_{1}, \ldots, a_{j}$. Класс $\Pi_{n}^{i}(\vec{a})$ определяется аналогично, а $\Delta_{n}^{i}(\vec{a})=\Sigma_{n}^{i}(\vec{a}) \cap \Pi_{n}^{i}(\vec{a})$. В частных случаях $j=1, j=0$ пишут $\Sigma_{n}^{i}(a)$ и $\Sigma_{n}^{i}$, и то же для $\Pi$ и $\Delta$. Дополнительно, определяют $\Sigma_{n}^{i}(P)=\bigcup_{a_{1}, \ldots, a_{j} \in P \cap \mathbb{N} \omega} \Sigma_{n}^{i}\left(a_{1}, \ldots, a_{j}\right)$ для каждого множества $P$, и аналогично $\Pi_{n}^{i}(P), \Delta_{n}^{i}(P)$. Если множество $P \cap \mathbb{N}^{\omega}$ рекурсивно замкнуто, то $\Sigma_{n}^{i}(P)$ совпадает с $\bigcup_{a \in P \cap \mathbb{N} \omega} \Sigma_{n}^{i}(a)$, и то же для $\Pi, \Delta$.

Классы, обозначаемые с использованием букв $\Sigma, \Pi, \Delta$, будут назьваться әффективнылми, в отличие от проективных классов $\Sigma_{n}^{1}, \boldsymbol{\Pi}_{n}^{1}, \boldsymbol{\Delta}_{n}^{1}$. Однако проективная иерархия является лишш частным случаем эффективной:

ПРЕДЛОЖЕНИЕ 1.5. $\boldsymbol{\Sigma}_{n}^{i}=\Sigma_{n}^{i}\left(\mathbb{N}^{\omega}\right)$, m.e. $X \in \boldsymbol{\Sigma}_{n}^{i}$ тогда и только тогда, когда $X \in \Sigma_{n}^{i}(a)$ для какого-нибудь $a \in \mathbb{N}^{\omega}$, и то жее для классов $\boldsymbol{\Pi}, \boldsymbol{\Delta}$.

ДокАЗАТЕльство. Множества, определимые $\Sigma_{1}^{0}$-формулами в пространствах $\mathscr{N}^{(k, \ell)}$, очевидно, открыты, так что $\Sigma_{1}^{0}\left(\mathbb{N}^{\omega}\right) \subseteq \Sigma_{1}^{0}$. Обратно, если $X$ открыто, скажем, в $\mathbb{N}^{\omega}$, то $X=\bigcup_{n} \mathscr{N}_{s_{n}}$, где $s_{n} \in \mathbb{N}^{<\omega}$, т.е. $X$ определяется $\Sigma_{1}^{0}$-формулой $\exists n \forall m<a(n) \quad\left(x(m)=b\left(2^{n}(2 m+1)-1\right)\right)($ с параметрами $a, b)$, где $a(n)=$ lh $s_{n}$ (длина конечной последовательности $\left.s_{n}\right)$, и $b\left(2^{n}(2 m+1)-1\right)=s_{n}(m)$ при $m<\operatorname{lh} s_{n}$ и равно 0 при $m \geqslant \operatorname{lh} s_{n}$. Итак, $\Sigma_{1}^{0}\left(\mathbb{N}^{\omega}\right)=\Sigma_{1}^{0}$. Отсюда следует искомьй результат для замкнутых множеств, $\mathbf{F}_{\sigma}$ и $\mathbf{G}_{\delta}$, а также тот факт, что арифметические формулы (с параметрами) определяют борелевские множества конечного ранга. Что до проективных классов, квантор $\exists x$ (т.е., в наших соглашениях, $\exists x \in \mathbb{N}^{\omega}$ ) соответствует проекции, а квантор $\forall x$ - комбинации “дополнение - проекция дополнение". 
16

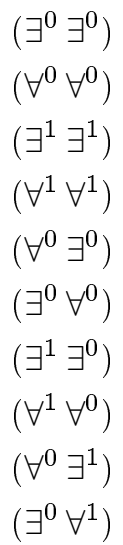

В.Г. КАНОВЕЙ, В.А. ЛЮБЕЦКИЙ

$$
\begin{aligned}
\exists i \exists j \varphi(i, j) & \Longleftrightarrow \exists n \varphi\left((n)_{1},(n)_{2}\right) \\
\forall i \forall j \varphi(i, j) & \Longleftrightarrow \forall n \varphi\left((n)_{1},(n)_{2}\right) \\
\exists x \exists y \varphi(x, y) & \Longleftrightarrow \exists z \varphi\left((z)_{1},(z)_{2}\right) \\
\forall x \forall y \varphi(x, y) & \Longleftrightarrow \forall z \varphi\left((z)_{1},(z)_{2}\right) \\
\forall i \exists j \varphi(i, j) & \Longleftrightarrow \exists x \forall i \varphi(i, x(i)) \\
\exists i \forall j \varphi(i, j) & \Longleftrightarrow \forall x \exists i \varphi(i, x(i)) \\
\exists x \exists j \varphi(x, j) & \Longleftrightarrow \exists y \varphi\left((y)_{0},(y)_{1}(0)\right) \\
\forall x \forall j \varphi(x, j) & \Longleftrightarrow \forall y \varphi\left((y)_{0},(y)_{1}(0)\right) \\
\forall i \exists x \varphi(i, x) & \Longleftrightarrow \exists x \forall i \varphi\left(i,(x)_{i}\right) \\
\exists i \forall x \varphi(i, x) & \Longleftrightarrow \forall x \exists i \varphi\left(i,(x)_{i}\right)
\end{aligned}
$$

ТАБлицА. Отображение $\langle i, j\rangle \mapsto 2^{i}(2 j+1)-1$ является биекцией $\mathbb{N}^{2}$ на $\mathbb{N}$. Через $(n)_{1}$ и $(n)_{2}$ обозначаются обратные функции, т.е. $(n)_{1}=i$ и $(n)_{2}=j$ при $2^{i}(2 j+1)-1=n$. Для $z \in \mathbb{N}^{\omega}$ и $n \in \mathbb{N}(z)_{n} \in \mathbb{N}^{\omega}$ определено в п. $1 \mathrm{~A}$

Преобразование аналитических формул. Эквивалентности в таблице позволяют, за счет упрощения кванторной приставки, приводить сложные аналитические формулы к такому виду, который сразу дает возможность судить о типе определяемого множества. Заметим, что предпоследняя эквивалентность $\left(\forall^{0} \exists^{1}\right)$ выражает счетную аксиому выбора, а $\left(\exists^{0} \forall^{1}\right)$ - двойственное утверждение.

В качестве элементарного примера заметим следуюшее: ecлu $\varphi(x, y, i, j)$ ecmь $\Sigma_{n}^{1}$-формула, $n \geqslant 1$, то әтому же типу принадлежсат и, скажем, формуль $\exists x \varphi(x, y, i, j), \exists i \varphi(x, y, i, j), \forall i \varphi(x, y, i, j)$ : в том смысле, что они приводятся к $\Sigma_{n}^{1}$-виду (с теми же параметрами) при помоши правил $\left(\exists^{1} \exists^{1}\right),\left(\exists^{1} \exists^{0}\right),\left(\forall^{0} \exists^{1}\right)$.

1Г. Элементы теории $\Pi_{1}^{1}$-множеств. Напомним, что решетом над пространством $\mathbb{X}$ назьвается всякое множество $R \subseteq \mathbb{X} \times \mathbb{Q}$ (где $\mathbb{Q}$ - рациональные числа) или, что равносильно, система $\left\{R_{q}\right\}_{q \in \mathbb{Q}}$ множеств $R_{q}=\{x \in \mathbb{X}:\langle x, q\rangle \in R\} \subseteq \mathbb{X}$, называемых әлементами $R$. При этом каждой точке $x \in \mathbb{X}$ сопоставляется множество $R^{(x)}=\left\{q: x \in R_{q}\right\} \subseteq \mathbb{Q}$, что разбивает пространство $\mathbb{X}$ на два множества:

$$
\begin{aligned}
& E(R)=\left\{x: R^{(x)} \text { вполне упорядочено }\right\} \quad \text { - внешнее множество, } \\
& \mathscr{E}(R)=\left\{x: R^{(x)} \text { не вполне упорядочено }\right\}-\text { внутреннее множество }
\end{aligned}
$$

(вполне упорядоченность понимается в смысле естественного порядка рациональных чисел), которые разбиваются на конституанты

$$
E_{\xi}(R)=\left\{x \in E: \operatorname{otp} R^{(x)}=\xi\right\} \quad \text { и } \quad \mathscr{E}_{\xi}(R)=\left\{x \in \mathscr{E}: \operatorname{otp} \operatorname{MIS}\left(R^{(x)}\right)=\xi\right\}
$$

$\left(\xi<\omega_{1}\right)$, где otp $S$ - порядковый тип $S \subseteq \mathbb{Q}$ (если $S$ вполне упорядочено), a $\operatorname{MIS}(S)$ наибольший вполне упорядоченньй начальньй сегмент $S$.

Зафиксируем раз навсег да рекурсивную биекцию $n \mapsto q_{n}: \mathbb{N} \stackrel{\text { на }}{\longrightarrow} \mathbb{Q}$, и пусть $q \mapsto n_{q}$ - обратна биекция. Этим индуцируется гомеоморфизм $\mathbb{N}^{\omega} \times \mathbb{Q}$ на $\mathbb{N}^{\omega} \times \mathbb{N}$, что позволяет классифицировать решета в терминах проективной иерархии, так что решето класса $K$ над $\mathbb{X}=\mathscr{N}^{(k, \ell)}$ - это множество $R \subseteq \mathbb{X} \times \mathbb{Q}$ класса $K$. Для борелевских или проективных классов $\mathbf{K}=\boldsymbol{\Sigma}_{n}^{i}, \boldsymbol{\Pi}_{n}^{i}, \boldsymbol{\Delta}_{n}^{i}$ это равносильно тому, что каждьй элемент $R_{q}$ решета $R$ принадлежит $\mathbf{K}$. 
Теорема 1.6 (теорема просеивания). Пусть $\mathbb{X}=\mathscr{N}^{(k, \ell)}$.

(а) Если $R \subseteq \mathbb{X} \times \mathbb{Q}$ - борелевское решето, то множсество $E(R)$ принадлежит $\Pi_{1}^{1}$, а все конституанты $E_{\xi}(R)$ и $\mathscr{E}_{\xi}(R)$ - борелевские множества. Обратно, для любого $\Pi_{1}^{1}$-множества $X \subseteq \mathbb{X}$ найдется решето $R \subseteq \mathbb{X} \times \mathbb{Q}$ класса $\Delta_{1}^{0}$, т.е. с открыто-замкнутыми әлементами, такое, что $X=E(R)$.

(б) (эффективный вариант теоремы) Ecли $a \in \mathbb{N}^{\omega} u R \subseteq \mathbb{X} \times \mathbb{Q}$ - решето класса $\Delta_{1}^{1}(a)$, то $E(R)$ есть $\Pi_{1}^{1}(a)$-множество. Обратно, для любого $\Pi_{1}^{1}(a)$-множества $X \subseteq \mathbb{X}$ найдется $\Delta_{1}^{0}($ a)-решето $R \subseteq \mathbb{X} \times \mathbb{Q}$ такое, ито $X=E(R)$.

ДокАЗАТЕЛЬСтво. Для произвольного решета $R \subseteq \mathbb{N}^{\omega} \times \mathbb{Q}$ мы имеем

$$
x \in E(R) \Longleftrightarrow \forall f: \mathbb{N} \rightarrow \mathbb{Q}(f \text { монотонно убывает } \Longrightarrow \exists n(\langle x, f(n)\rangle \notin R)),
$$

поэтому если $R$ - решето класса хотя бы $\Sigma_{1}^{1}(a)$, то правая часть легко приводится к $\Pi_{1}^{1}$-виду с параметром $а$ с помощью преобразований из таблицы п. $1 \mathrm{~B}$ и фиксированной вьше рекурсивной биекции $n \mapsto q_{n}$ для замены $\mathbb{Q}$ на $\mathbb{N}$.

Обратно, рассмотрим $\Pi_{1}^{1}(a)$-множество $X=\left\{x \in \mathbb{N}^{\omega}: \forall y \varphi(x, y, a)\right\}$, где $\varphi$ - арифметическая формула вида П $\psi(x, y, a, \ldots)$, где П - блок кванторов вида $\exists n, \forall n$ (типа 0), а $\psi$ - ограниченная формула. Пусть, к примеру, $\varphi$ есть $\exists i \forall j \exists k \psi(x, y, a, i, j, k)$. Правило $\left(\exists^{0} \forall^{1}\right)$ таблицы п. 1В преобразует это к виду $\forall z \exists i \exists k \psi(x, y, a, i, z(i), k)$, а другие правила этой таблицы приводят определение $X$ к виду $X=\left\{x \in \mathbb{N}^{\omega}\right.$ : $\forall y \exists m \Phi(x, y, a, m)\}$, где $\Phi-$ ограниченная формула.

В силу ограниченности $\Phi$, определение истинности $\Phi(x, y, a, m)$ можно представить как работу компьютерной программы (зависящей лишш от структуры $\Phi$, но не от аргументов $x, y, a, m)$, куда $x, y, a$ вводятся как бесконечные ленты значений $x(n), y(n)$, $a(n), n \in \mathbb{N}$, а результат получается после конечного числа шагов. Если $s \in \mathbb{N}<\omega$, то пусть $s^{\wedge} \mathbf{0} \in \mathbb{N}^{\omega}-$ продолжение $s$ нулями. Положим

$S^{x a}=$ множество всех $s \in \mathbb{N}<\omega$ таких, что для любого $m<\operatorname{lh} s$ если вычисление $\Phi\left(x, s^{\wedge} \mathbf{0}, a, m\right)$ проходит без обрашения к значениям $\left(s^{\wedge} \mathbf{0}\right)(k), k \geqslant \operatorname{lh} s$, то результат вьгисления - “ложь” (т.е. $\left.\neg \Phi\left(x, s^{\wedge} \mathbf{0}, a, m\right)\right)$.

Для $s, t \in \mathbb{N}<\omega$ положим $s<_{\mathrm{LS}} t$, когда либо $t \subset s$, либо $s<t$ лексикографически (порядок Лузина-Серпинского). Нетрудно проверить, что

$$
\begin{aligned}
x \in X & \Longleftrightarrow S^{x a} \text { не имеет бесконечных путей } \\
& \Longleftrightarrow S^{x a} \text { вполне упорядочено в смысле }<_{\mathrm{LS}} .
\end{aligned}
$$

Однако $<_{\text {LS }}$ - счетный плотный в себе линейньй порядок с наибольшим элементом, но без наименьшего. Зафиксируем рекурсивную биекцию $f: \mathbb{N}<\omega \stackrel{\text { на }}{\longrightarrow} \mathbb{Q} \leqslant 1=\{q \in \mathbb{Q}$ : $q \leqslant 1\}$, сохраняюшую порядок, т.е. $s<_{\mathrm{LS}} t \Longleftrightarrow f(s)<f(t)$. Согласно вьшедоказанному, решето $R=\left\{\langle x, f(s)\rangle: s \in S^{x a}\right\}$ удовлетворяет $X=E(R)$. Остается заметить, что $R$ - решето класса $\Delta_{1}^{0}(a)$ : правая часть определения $S^{x a}$, как формула с переменными $x$ и $s$, приводится как к $\Sigma_{1}^{0}(a)$-виду (с внешним квантором "сушествует вычисление”), так и к $\Pi_{1}^{0}(a)$-виду (“для каждого вычисления").

Борелевость конституант есть классический результат.

СлЕДСТВИЕ 1.7. Каждое $\boldsymbol{\Sigma}_{2}^{1}$-множество является облединением борелевских множеств в числе $\aleph_{1}$. 
ДокАЗАТЕльство. По теореме просеивания, каждое $\boldsymbol{\Pi}_{1}^{1}$-множество есть объединение $\aleph_{1}$ борелевских множеств, следовательно, каждое $\boldsymbol{\Sigma}_{2}^{1}$-множество есть объединение $\aleph_{1}$ проекиий борелевских множеств, т.е. $\aleph_{1} \boldsymbol{\Sigma}_{1}^{1}$-множеств. Но каждое из последних - снова объединение $\aleph_{1}$ борелевских множеств по теореме 1.6.

Теорема 1.8. Пусть $R$ - борелевское решето над пространством $\mathscr{N}^{(k, \ell)}$.

(i) Если $\Sigma_{1}^{1}$-множество $А$ включено в $E(R)$, то найдется ординал $\vartheta<\omega_{1}$ такой, что $A \subseteq \bigcup_{\xi<\vartheta} E_{\xi}(R)$.

(ii) Множество $E(R)$ является борелевским в том и только том случае, когда найдется ординал $\vartheta<\omega_{1}$ такой, что $E_{\xi}(R)=\varnothing$ для всех $\xi>\vartheta$.

(iii) Класс $\Delta_{1}^{1}$ тождествен классу всех борелевских множеств.

Униформизашия. Пусть $\mathbb{X}, \mathbb{Y}$ - произвольные пространства. Если $P \subseteq \mathbb{X} \times \mathbb{Y}$, то часто пишут $P(x, y)$ вместо $\langle x, y\rangle \in P$. Множество $\operatorname{dom} P=\{x: \exists y \quad P(x, y)\} \subseteq \mathbb{X}$ называется проекиией $P$ (на $\mathbb{X})$.

Множество $P \subseteq \mathbb{X} \times \mathbb{Y}$ называется униформным, если для любого $x \in \mathbb{X}$ сушествует не более одного $y \in Y$ такого, что $P(x, y)$; это означает, собственно говоря, что $P$ график частичной функции $\mathbb{X} \rightarrow \mathbb{Y}$. Если $P \subseteq Q \subseteq \mathbb{X} \times \mathbb{Y}, P$ униформно и $\operatorname{dom} P=$ $\operatorname{dom} Q$, то пишут, что $P$ униформизует $Q$.

Теорема 1.9 (теорема Новикова-Кондо-Аддисона, [62], [43] или [86; §7.11]). Каждое множество $P \subseteq \mathbb{N}^{\omega} \times \mathbb{N}^{\omega}$ класса $\Pi_{1}^{1}$ можно униформизовать множеством того же класса. Каждое множество $P \subseteq \mathbb{N}^{\omega} \times \mathbb{N}^{\omega}$ класса $\Pi_{1}^{1}(a)$ $\left(a \in \mathbb{N}^{\omega}\right)$ можсно униформизовать множсеством того же класса.

1Д. Отступление: кодировка точками бэровского пространства. Элементы пространства $\mathbb{N}^{\omega}$ могут быть использованы для кодировки математических объектов самой различной природы, характеризуемых тем свойством, что они, неформально говоря, содержат лишь счетное число битов информации.

ПримеР 1.10 .1 (кодировка счетных ординалов). Напомним, что $q \mapsto n_{q}$-биекция $\mathbb{Q}$ на $\mathbb{N}$. Обозначим через $\mathbf{W O}$ множество всех $w \in \mathbb{N}^{\omega}$ таких, что $Q_{w}=\{q \in \mathbb{Q}$ : $\left.w\left(n_{q}\right)=0\right\}$ вполне упорядочено порядком $\mathbb{Q}$, и пусть в этом случае $|w|=\operatorname{otp} Q_{w}$ есть порядковый тип $Q_{w}$, т.е. $|w|<\omega_{1}$.

Положим $\mathbf{W} \mathbf{O}_{\gamma}=\{w \in \mathbf{W O}:|w|=\gamma\}$.

Эта полезная кодировка счетных ординалов допускает простое истолкование в терминах решет. Положим $\mathscr{L}_{q}=\left\{x \in \mathbb{N}^{\omega}: x\left(n_{q}\right)=0\right\}$. Этим задано бинарное решето Лебега $\mathscr{L}=\left\{\mathscr{L}_{q}\right\}_{q \in \mathbb{Q}}$. В этом случае $\Pi_{1}^{1}$-множество $E(\mathscr{L})$, очевидно, совпадает с $\mathbf{W O}$, a $\mathbf{W} \mathbf{O}_{\gamma}=E_{\gamma}(\mathscr{L})$ для всех $\gamma<\omega_{1}$.

Предположим, что $w \in \mathbf{W O}$ и $k \in \mathbb{N}, w(k)=0$. Определим $w \uparrow_{k} \in \mathbf{W O}$ следуюшим образом: $w \uparrow_{k}(n)=0$ при $w(n)=0$ и $q_{n}<q_{k}$ и $w \uparrow_{k}(n)=1$ во всех остальных случаях. Ясно, что $\{\xi: \xi<|w|\}=\left\{\left|w \uparrow_{k}\right|: w(k)=0\right\}$.

ПримеР 1.10 .2 (кодировка борелевских множеств). Фиксируем раз навсегда рекурсивное перечисление без повторений $\mathbb{N}<\omega=\left\{s_{k}: k \in \mathbb{N}\right\}$.

Положим $\mathbf{B C}_{0}=\{\boldsymbol{k}: k \in \mathbb{N}\}$, где $\boldsymbol{k} \in \mathbb{N}^{\omega}$ есть функция-константа (т.е. $\boldsymbol{k}(i)=k$ для всех $i$ ). Для $\boldsymbol{k} \in \mathbf{B C}_{0}$ определим $\boldsymbol{B}_{\boldsymbol{k}}=\mathscr{N}_{s_{k-1}}$ (бэровский интервал в $\mathbb{N} \omega$, см. п. $\left.1 \mathrm{~A}\right)$ при $k \geqslant 1$ и $\boldsymbol{B}_{\boldsymbol{k}}=\varnothing$ при $k=0$. Если $\xi>0$, то определим $\mathbf{B C}_{\xi}$ как совокупность всех $c \in \mathbb{N}^{\omega} \backslash \bigcup_{\eta<\xi} \mathbf{B C}_{\eta}$ таких, что $\left\{(c)_{n}: n \in \mathbb{N}\right\} \subseteq \bigcup_{\eta<\xi} \mathbf{B C}_{\eta}$; полагаем $\boldsymbol{B}_{c}=$ $\mathbb{N}^{\omega} \backslash \bigcup_{n} \boldsymbol{B}_{(c)_{n}}$ для всякого такого $c$. (Определение $(c)_{n}$ см. в п. 1А.) Этим определено 
множество $\mathbf{B C}=\bigcup_{\xi<\omega_{1}} \mathbf{B C}_{\xi} \subseteq \mathbb{N}^{\omega}$ борелевских кодов и борелевское множество $\boldsymbol{B}_{c} \subseteq \mathbb{N}^{\omega}$ для каждого $c \in \mathbf{B C}^{16}$

ЗАмЕЧАНИЕ 1.10.3. На построение $\mathbf{B C}$ и $\boldsymbol{B}_{c}$ можно взглянуть с другой стороны. Положим $(c)_{s}=\left(\ldots\left((c)_{s(0)}\right)_{s(1)} \ldots\right)_{s(n-1)}$ для всех $c \in \mathbb{N}^{\omega}, s \in \mathbb{N}<\omega, n=\operatorname{lh} s$ (отдельно, $(c)_{\Lambda}=x$ для пустой последовательности $\left.\Lambda\right)$. Нетрудно проверить, что

$$
T_{c}=\left\{s \in \mathbb{N}^{<\omega}: \forall n<\operatorname{lh} s\left((c)_{s \uparrow n} \text { не является константой }\right)\right\}
$$

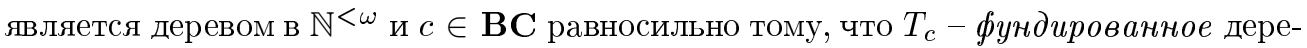
во, т.е. оно не имеет бесконечных путей, кроме того, $s \in T_{c}$ - максимальньй элемент $T_{c}$ в том и только том случае, когда $(c)_{s}-$ константа. Далее, если $c \in \mathbf{B C}$, то мы можем определить борелевское множество $B_{c}(s) \subseteq \mathbb{N}^{\omega}$ для каждой последовательности $s \in T_{c}$ так, что $B_{c}(s)=\mathscr{N}_{s_{k-1}}$ при $(c)_{s}=k$ (константа $k$ ) и $k \geqslant 1, B_{c}(s)=\varnothing$ при $(c)_{s}=k$ и $k=0$, а если $(c)_{s}$ не константа, т.е. $s-$ не максимальньй элемент $T_{c}$, то $B_{c}(s)=\mathbb{N}^{\omega} \backslash \bigcup_{n} B_{c}\left(s^{\wedge} n\right)$ - и при этом, как нетрудно проверить, получается $B_{c}(\Lambda)=\boldsymbol{B}_{c}$, вообще, $B_{c}(s)=\boldsymbol{B}_{(c)}$ для $s \in T_{c}$.

При задании кодировки каких-либо математических структур объектами более простого типа наиболее интересный вопрос состоит в следуюшем: каковы свойства тех отношений на кодирующих объектах, которые соответствуют главньм отношениям между самими кодируемыми структурами. В нашем случае наиболее важньм является вопрос об определимости так индуцированных отношений. Следуюшее предложение содержит наиболее важные результаты в этом плане.

ПРЕДЛОЖЕНИЕ 1.11.

(i) Множества WO, BC принадлежат $\Pi_{1}^{1}$, точнее, каждое из них определимо, в ZFC, некоторой явно заданной $\Pi_{1}^{1}$-формулой.

(ii) Найдутся $\Sigma_{1}^{1}$-формула $\sigma(\cdot, \cdot)$ и $\Pi_{1}^{1}$-формула $\pi(\cdot, \cdot)$ такие, что при любьх $w, z \in \mathbf{W O}$ вьполнено $|w| \leqslant|z| \Longleftrightarrow \sigma(w, z) \Longleftrightarrow \pi(w, z)$. То же для $<,=$

(iii) Если $R$ - решето класса $\Delta_{1}^{1}(a), a \in \mathbb{N}^{\omega}$, то найдутся $\Sigma_{1}^{1}(a)$-форлульи (т.е. $\Sigma_{1}^{1}$-формулы с единственным параметром $\left.a\right) \sigma(\cdot, \cdot), \sigma^{\prime}(\cdot, \cdot)$ u $\Pi_{1}^{1}(a)$-формуль $\pi(\cdot, \cdot), \pi^{\prime}(\cdot, \cdot)$ такие, что при любьх $w \in \mathbf{W O}$ и $x \in \mathbb{N}^{\omega}$ выполнено

$$
\begin{aligned}
& x \in E_{|w|}(R) \Longleftrightarrow \sigma(w, x) \Longleftrightarrow \pi(w, x), \\
& x \in \mathscr{E}_{|w|}(R) \Longleftrightarrow \sigma^{\prime}(w, x) \Longleftrightarrow \pi^{\prime}(w, x) .
\end{aligned}
$$

(iv) Найдутся $\Sigma_{1}^{1}$-формула $\sigma(\cdot, \cdot)$ и $\Pi_{1}^{1}$-формула $\pi(\cdot, \cdot)$ такие, что при любьх $c \in \mathbf{B C} u x \in \mathbb{N}^{\omega}$ выполнено $x \in \boldsymbol{B}_{c} \Longleftrightarrow \sigma(c, x) \Longleftrightarrow \pi(c, x)$.

(v) Найдутся $\Sigma_{1}^{1}$-формула $\varphi_{\boldsymbol{\lambda}}(\cdot, \cdot, \cdot)$ и $\Pi_{1}^{1}$-формула $\psi_{\boldsymbol{\lambda}}(\cdot, \cdot, \cdot)$ такие, что при любих с $\in \mathbf{B C}$ u $m, n \in \mathbb{N}$ выполнено $\boldsymbol{\lambda}\left(\boldsymbol{B}_{c}\right)=m / n \Longleftrightarrow \varphi_{\boldsymbol{\lambda}}(c, m, n) \Longleftrightarrow$ $\psi_{\boldsymbol{\lambda}}(c, m, n)$. (Мера $\boldsymbol{\lambda}$ на $2^{\omega}$ введена в п. 1Б.)

(vi) Найдутся $\Sigma_{1}^{1}$-формула $\varphi_{\text {саt }}(\cdot, \cdot)$ и $\Pi_{1}^{1}$-формула $\psi_{\text {cat }}(\cdot, \cdot)$ такие, что при любых $с \in \mathbf{B C} u k \in \mathbb{N}$ выполнено: $\boldsymbol{B}_{c} \cap \mathcal{N}_{s_{k}}$ тощее $\Longleftrightarrow \varphi_{\text {cat }}(c, k) \Longleftrightarrow$ $\psi_{\text {cat }}(c, k) .\left(\left\{s_{k}\right\}_{k \in \mathbb{N}}-\right.$ фиксированное рекурсивное перечисление $\left.\mathbb{N}<\omega.\right)$

\footnotetext{
${ }^{16} \mathrm{~B}$ основе этой кодировки лежит операция дополнения к счетному объединению. Через нее легко выражается само дополнение (кодом дополнения $\boldsymbol{B}_{c}$ может служить $c^{\prime} \in \mathbb{N}^{\omega}$, определенное так, что $\left.\left(c^{\prime}\right)_{n}=c, \forall n\right)$, следовательно, также операции счетного объединения и пересечения. Тем самьм, каждое борелевское множество $X \subseteq \mathbb{N}^{\omega}$ имеет вид $\boldsymbol{B}_{c}$ для подходящего $c \in \mathbf{B C}$.
} 
ДоказАТЕЛЬство. (i) $w \in \mathbf{W O}$ равносильно несуществованию бесконечно убываюшей цепочки элементов $Q_{w}$ (в терминологии примера 1.10.1), а это выразимо $\Pi_{1}^{1}$ формулой. Далее, $c \in \mathbf{B C}$ равносильно тому, что для любого $a \in \mathbb{N}^{\omega}$ имеется $n$ такое, что $(s)_{a \nmid n} \notin T_{c}$ (в терминах замечания 1.10.3).

(ii) При $w, z \in \mathbf{W O}|w| \leqslant|z|$ равносильно сушествованию порядкового изоморфизма множества $Q_{w}$ на начальньй отрезок $Q_{z}$ и несушествованию порядкового изоморфизма $Q_{z}$ на собственный начальный отрезок $Q_{w}$. Такие порядковые изоморфизмыне принадлежат $\mathbb{N}^{\omega}$, но легко кодируются точками $\mathbb{N}^{\omega}$.

(iii) $x \in E_{|w|}(R)$ равносильно существованию порядкового изоморфизма между множествами $Q_{w}$ и $R^{(x)}$ и несуществованию такового между $Q_{w}$ и собственным начальным сегментом $R^{(x)}$ или наоборот - это приносит искомые формулы $\sigma, \pi$. Формулы для $\mathscr{E}_{|w|}(R)$ получаются на основе аналогичных простых соображений.

(iv) При $c, x \in \mathbb{N}^{\omega}$ назовем $c, x$-функиией любую функцию $f: \mathbb{N}<\omega \rightarrow\{0,1\}$ такую, что для всякого $s \in \mathbb{N}<\omega$ если $(c)_{s}$ есть функция-константа $\boldsymbol{k}$ для какого-либо $k$, то $f(s)=1 \Longleftrightarrow x \in \mathscr{N}_{s_{k-1}}$ при $k \geqslant 1, f(s)=0$ при $k=0$, а если $(c)_{s}$ не константа, то $f(s)=1 \Longleftrightarrow \forall m\left(f\left(s^{\wedge} m\right)=0\right)$. Понятно, что $c, x$-функция $f=f_{c x}$ сушествует и единственна при $c \in \mathbf{B C}$, причем $x \in \boldsymbol{B}_{c}$ равносильно $f_{c x}(\Lambda)=1$. Тем самьм, $x \in \boldsymbol{B}_{c}$ равносильно (при $c \in \mathbf{B C}$ ) существованию $c, x$-функции $f$ с $f(\Lambda)=1$ и несуществованию $c, x$-функции $f$ с $f(\Lambda)=0$.

(v) Назовем $\boldsymbol{\lambda}$-аппрожсимацией множества $X \subseteq \mathbb{N}^{\omega}$ пару $\langle U, V\rangle \mathbf{F}_{\sigma}$-множеств такую, что $U \subseteq X, V \cap X=\varnothing$ и $\boldsymbol{\lambda}(U \cup V)=1$. Понятно, что в этом случае $\boldsymbol{\lambda}(U)=\boldsymbol{\lambda}(X)$. Отметим, что если $\left\langle U_{n}, V_{n}\right\rangle$ являются $\lambda$-аппроксимациями множеств $X_{n}$, то естественная идея взять $\left\langle V=\bigcap_{n} V_{n}, U=\bigcup_{n} U_{n}\right\rangle$ в качестве $\boldsymbol{\lambda}$-аппроксимации множества $X=\mathbb{N}^{\omega} \backslash \bigcup_{n} X_{n}$ не проходит: $V$ не обязательно будет $\mathbf{F}_{\sigma}$-множеством. Можно, однако, поступить иначе. Пусть $V_{n}=\bigcup_{k} V_{n k} \forall n$, где все $V_{n k}$ замкнуты. Для любого заданного $j$ выберем в каждом множестве $V_{n}$ замкнутое $V_{n j}^{\prime}=\bigcup_{k<k(n, j)} V_{n k} \subseteq V_{n}$ так, что $V_{n j}^{\prime} \subseteq V_{n, j+1}^{\prime}$ и $\boldsymbol{\lambda}\left(V_{n} \backslash V_{n j}^{\prime}\right) \leqslant 2^{-(n+j)}$. Тогда $V_{j}^{\prime}=\bigcap_{n} V_{n j}^{\prime}$ - замкнутое подмножество $V$ с $\boldsymbol{\lambda}\left(V \backslash V_{j}^{\prime}\right) \leqslant \sum_{n} 2^{-(n+j)}=2^{-j+1}$. Следовательно, $V^{\prime}=\bigcup_{j} V_{j}^{\prime}$

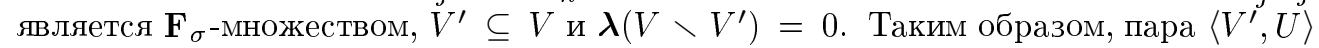
является $\lambda$-аппроксимацией множества $X$. Пусть $\Phi\left(V^{\prime}, U,\left\{U_{n k}\right\}_{n, k \in \mathbb{N}},\left\{V_{n k}\right\}_{n, k \in \mathbb{N}}\right)$ выражает тот факт, что $\mathbf{F}_{\sigma}$-множество $V^{\prime}$ получилось из замкнутых множеств $V_{n k}$ указанным способом, а $U=\bigcup_{n, k \in \mathbb{N}} U_{n k}$.

Теперь используем эту конструкцию для построения формул $\varphi_{\boldsymbol{\lambda}}, \psi_{\boldsymbol{\lambda}}$. Здесь нам по-

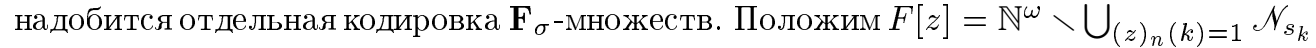
и $F_{\sigma}[z]=\bigcup_{n} F\left[(z)_{n}\right]$ для любого $z \in \mathbb{N}^{\omega}$. Назовем кодом аппроксимации для $c \in \mathbb{N}^{\omega}$ любую пару отображений $\gamma, \delta: \mathbb{N}<\omega \rightarrow \mathbb{N} \omega$, удовлетворяюшую таким требованиям:

(1) если $s \in \mathbb{N}^{\omega}$ таково, что $(c)_{s}=k$ и $k \geqslant 1$, то, во-первых, $(\gamma(s))_{n}(j)=1$ для всех $n$ и всех $j$ таких, что $\mathscr{N}_{j} \cap \mathscr{N}_{k-1}=\varnothing$, но $(\delta(s))_{n}(j)=0$ при $\mathscr{N}_{j} \cap \mathscr{N}_{k-1} \neq \varnothing$ - тогда, очевидно, $F_{\sigma}[\delta(s)]=\mathscr{N}_{s_{k-1}}=B_{c}(s)$, и, во-вторых, $(\delta(s))_{n}(k-1)=1$ и $(\delta(s))_{n}\left(k^{\prime}\right)=0$ при $k^{\prime} \neq k-1$ - тогда $F_{\sigma}[\delta(s)]=\mathbb{N}^{\omega} \backslash \mathscr{N}_{s_{k-1}}$;

(2) если $s \in \mathbb{N}^{\omega},(c)_{s}=k$ и $k=0$, то $(\gamma(s))_{n}(j)=1, \forall j-$ тогда $F_{\sigma}[\gamma(s)]=\varnothing=$ $B_{c}(s)$, и $(\delta(s))_{n}(j)=0, \forall j-$ тогда $F_{\sigma}[\delta(s)]=\mathbb{N}^{\omega}$

(3) если же $(c)_{s}$ не является константой, то $\Phi\left(V^{\prime}, U,\left\{U_{n k}\right\}_{n, k \in \mathbb{N}},\left\{V_{n k}\right\}_{n, k \in \mathbb{N}}\right)$, где $V^{\prime}=F_{\sigma}[\gamma(s)], U=F_{\sigma}[\delta(s)], U_{n k}=F\left[\left(\gamma\left(s^{\wedge} n\right)\right)_{k}\right], V_{n k}=F\left[\left(\delta\left(s^{\wedge} n\right)\right)_{k}\right]$.

Легко видеть, что для любого борелевского кода $c \in \mathbf{B C}$ существуют коды аппроксимации $\langle\gamma, \delta\rangle$ и для любого из них, при любом $s \in T_{c}$, пара $\left\langle F_{\sigma}[\gamma(s)], F_{\sigma}[\delta(s)]\right\rangle$ 
являетс $\boldsymbol{\lambda}$-аппроксимацией множества $B_{c}(s)$, в частности, $\left\langle F_{\sigma}[\gamma(\Lambda)], F_{\sigma}[\delta(\Lambda)]\right\rangle$ есть $\boldsymbol{\lambda}$-аппроксимация множества $B_{c}(\Lambda)=\boldsymbol{B}_{c}$. Тем самым, формулы

$$
\begin{aligned}
& \varphi_{\boldsymbol{\lambda}}(c, m, n):=\exists\langle\gamma, \delta\rangle\left(\langle\gamma, \delta\rangle-\text { код аппроксимации для } c \wedge \boldsymbol{\lambda}\left(F_{\sigma}[\gamma(\Lambda)]\right)=m / n\right), \\
& \psi_{\boldsymbol{\lambda}}(c, m, n):=\forall\langle\gamma, \delta\rangle\left(\langle\gamma, \delta\rangle-\text { код апшроксимации для } c \Longrightarrow \boldsymbol{\lambda}\left(F_{\sigma}[\gamma(\Lambda)]\right)=m / n\right)
\end{aligned}
$$

удовлетворяют эквивалентностям (v). С другой стороны, нетрудно проверить (хотя здесь это довольно длительная процедура, которую мы опускаем), что подформулы во внешних круглых скобках обеих формул являются арифметическими, так что эти формулы имеют нужный тип.

(vi) Здесь можно применить ту же самую конструкцию, только под аппроксимацией данного множества $X \subseteq \mathbb{N}^{\omega}$ следует теперь понимать пару $\langle U, F\rangle$, где $U \subseteq \mathbb{N}^{\omega}$ открыто, $F$ - тощее $\mathbf{F}_{\sigma}$-множество и $X \triangle U \subseteq F$. ( $\triangle$ обозначает симметрическую разность.) Главная техническая тонкость состоит в том, чтобы, имея апшроксимащии борелевских множеств $X_{n}$, определить аппроксимацию множества $X=\mathbb{N}^{\omega} \backslash \bigcup_{n} X_{n}$ - но она без труда решается.

Исторические и библиографические замечания. Множества (вещественной прямой) класса $\Sigma_{1}^{1}$ были введены Суслиньм [91] под названием: ensembles $(A)$. Они также известны как аналитические множества, суслинские множества и А-множества. Их предыстория прослеживается до заметки Александрова [3] и даже до мемуара Лебега [47], где дана конструкция (названная позже бинарнылм решетом Лебега, см. пример 1.10.1), из которой извлекается пример неборелевского П 1 -множества (об этом в [48]). История открытия А-операции и А-множеств стала предметом ряда специальных исследований; относяшиеся к этой теме факты, комментарии и дальнейшие ссылки читатель найдет, например, в [95], [50].

Свойство совершенного ядра для $\boldsymbol{\Sigma}_{1}^{1}$-множеств (теорема 1.4 ) связывается в литературе с именами Александрова, Хаусдорфа, Суслина (однако в соответствуюших работах [3], [23], [91] этой теоремы нет). Впервые результат появился со ссылкой на Суслина (но без указания конкретной работы) в [51]. (Более подробно в [97; § 3.3].) Теоремы об измеримости и свойстве Бэра для $\boldsymbol{\Sigma}_{1}^{1}$-множеств впервые появились также в [51].

Понятие решета было введено Лузиным в [55], но впервые появилось в контексте доказательства в [63] (а в несколько иной форме, в связи с А-операцией в [61]). Его систематическое применение в теории $\boldsymbol{\Sigma}_{1}^{1}$ - и $\boldsymbol{\Pi}_{1}^{1}$-множеств, включая теоремы 1.6 и 1.8 , дано в “Лекциях" [57]. Теорема 1.8 доказана в классических работах [5], [46], [57] (более современное изложение дано в [35]). Пункт (iii) (теорема Суслина) следует из (ii). Теорема униформизации 1.9 в ее первоначальной форме [62] утверждала, фактически, что $\Pi_{1}^{1}$-множества униформизуемы $\boldsymbol{\Sigma}_{2}^{1}$-множествами. Усиление до $\boldsymbol{\Pi}_{1}^{1}$ принадлежит Кондо [43], а результат для классов $\Pi_{1}^{1}(a)-$ Аддисону [1], [2] .

Проективные множества введены Лузиным [52] как “проективные множества Лебега", однако ссылка на Лебега здесь несостоятельна, об этом сказано в [97; 3.1$]$.

Систематическое использование аналитических формул для исследования проективных множеств (вместо геометрических построений, характерных для стиля Лузина и Новикова) было начато Аддисоном [1], [2]. Кодировка борелевских множеств была использована Соловеем [89] для решения проблемы измеримости проективных множеств, но ее корни (как и кодировки счетных ординалов) можно проследить до более ранних работ по теории рекурсии (например, в [86; 7.9]). Результаты (v) и (vi) предложения 1.11 восходят к работам конща 60-х годов, по проективньм множествам "с большими сечениями" см. ссылки в [41]. 
Главной современной ссылкой по классической (т.е. без форсинга, конструктивности и “эффективной" иерархии) дескриптивной теории множеств служит книга А.С. Кекриса [42]. Из более ранних работ, доступных на русском языке, можно отметить обзор [5], соответствующие главы из “Топологии” Куратовского [46], а также главу 8 в [11] и [35]. О проблемах классической дескриптивной теории говорится в обзорах [38], [40], [97].

\section{§. Конструктивность по Гёделю}

Классическая дескриптивная теория множеств остановилась перед вопросами о том, обладают ли все $\boldsymbol{\Pi}_{1}^{1}$-множества свойством совершенного ядра, измеримы ли все множества второго проективного уровня, имеют ли все они свойство Бэра. Дальнейшее изучение этих проблем стало возможным только после развития соответствуюших методов математической логики таких, как конструктивность и форсинг. С другой стороны, эти методы создавались, в значительной мере, как средства, направленные на решение классических проблем о точечных множествах.

Этот параграф̆ излагает исследования по тем аспектам конструктивности, которые существенны для исследования свойств регулярности проективных множеств.

2А. Теоретико-множественный универсум. Особенностью теории конструктивности Гёделя является то, что построения и доказательства в ней, в их наиболее общей и естественной форме, не замыкаются на чисто топологическую структуру точечных множеств, а напротив, аппеллируют к структуре теоретико-множественного универсума в целом и даже к взаимоотношениям разных универсумов, например, порождаемых форсингом.

Ординалы и ранги. Особое место в структре теоретико-множественного универсума занимают ординаль, или (конечные и трансфинитные) порядковые числа. Предполагая некоторое знакомство читателя с этими понятиями, напомним лишш, что в современной теории множеств каждый ординал $\xi$ отождествляется с множеством всех меньших ординалов, т.е. $\xi=\{\alpha: \alpha<\xi\}$. В частности, $0=\varnothing, 1=\{0\}, 2=\{0,1\}, \ldots$, $\omega=\mathbb{N}=\{0,1,2, \ldots\}, \omega+1=\omega \cup\{\omega\}$. Класс всех ординалов обозначается через Ord. Кардиналами же назьваются начальные ординалы, т.е. те, которые не равномощны меньшим ординалам. Например, $\omega=\mathbb{N}=\aleph_{0}-$ наименьший бесконечный ординал, $\omega_{1}=\aleph_{1}-$ наименьший несчетньй ординал и первьй несчетный кардинал ${ }^{17}$.

Напомним, что $\operatorname{rk} x \in \operatorname{Ord}$ обозначает теоретико-множсественный ранг $x$, определяемьй через $\operatorname{rk} x=\sup _{y \in x} \operatorname{rk} y$, где $\sup X$ есть наименшший ординал, строго больший всех ординалов из $X$. (Например, $\mathrm{rk} \varnothing=0$.) Отсюда производится иерархия фон Неймана, которая состоит из множеств $\mathbf{V}_{\xi}=\{x: \operatorname{rk} x<\xi\}, \xi \in$ Ord; универсум $\mathbf{V}$ всех множеств тождествен $\bigcup_{\xi \in \text { Ord }} \mathbf{V}_{\xi}$.

Модели аксиом ZFC. Мы предполагаем некоторое знакомство читателя с теорией множеств Цермело-Френкеля $\mathbf{Z F C}$ и не будем останавливаться на изложении ее аксиом (см. [11; гл. 1]). Как обычно, моделью ZFC назьвается любая структура вида $\langle\mathfrak{M} ; \varepsilon\rangle$, где $\mathfrak{M}$ - любое множество с бинарным отношением $\varepsilon$, в которой истинны все аксиомы ZFC (если знак принадлежности интерпретировать как $\varepsilon$ ). Выделяются

\footnotetext{
${ }^{17}$ Обозначения $\aleph_{0}, \aleph_{1}$, вообще $\aleph_{\xi}, \xi \in$ Ord, принято использовать в случае, когда важны лишь мощностные характеристики, обозначения $\omega, \omega_{1}$, вообще $\omega_{\xi}$ - когда важны порядковые характеристики кардинала и его связь с другими ординалами.
} 
стандартные транзитивные модели - это означает, что $\mathfrak{M}$ - транзитивное множество (т.е. $x \in y \in \mathfrak{M}$ влечет $x \in \mathfrak{M}$ ), а $\varepsilon$ - просто ограничение "настоящей" принадлежности $\in$ на $\mathfrak{M}$.

По теореме Гёделя о неполноте, существование хотя бы одной модели ZFC любого вида недоказуемо в ZFC. В то же время, существуют структуры вида $\langle\mathfrak{M} ; \varepsilon\rangle$, удовлетворяюшие всем аксиомам ZFC, но такие, что $\mathfrak{M}$ - не множество, а собственнвый класс. Простейший пример: $\langle\mathbf{V} ; \in\rangle$, где $\mathbf{V}$ - универсум всех множеств. Такие структуры - это не модели в строгом смысле ${ }^{18}$, поскольку их области не являются множествами. Поэтому к ним неприменима теорема Гёделя. Однако они чрезвьчайно полезны во многих приложениях: в частности, существование модели ZFC, даже в этом несобственном смысле, в которой истинно некоторое предложение $\Phi$, означает, что $\Phi$ не противоречит аксиомам ZFC.

K этой категории структур относится класс $\mathbf{L}$ всех конструктивных множеств, a также его расширения, получаемые при помоши метода вынуждения.

Наследственно счетные множества. Иногда приходится рассматривать модели более слабых теорий, в частности, теории $\mathbf{Z F C}^{-}$, т.е. $\mathbf{Z F C}$ без аксиомы степени. Естественной моделью $\mathbf{Z F C}-$ служит множество $\mathbf{H C}$ всех наследственно счетных множеств, т.е. таких $x$, что транзитивное замыкание ТС $(x)$ не более чем счетно. (ТС $(x)$ есть наименьшее транзитивное множество, содержашее $x$.) В частности, все натуральные числа и их множества, все элементы и счетные подмножества $\mathbb{N} \omega$, все счетные ординалы принадлежат $\mathbf{H C}$, однако, конечно, $\mathscr{P}(\mathbb{N}) \notin \mathbf{H C}$, и поэтому аксиома степени ложна в $\mathbf{H C}$. То, что все остальные аксиомы ZFC выполнены в $\mathbf{H C}$ (точнее говоря, в структуре $\langle\mathbf{H C} ; \in\rangle),-$ легкое упражнение. Теорема Скулема-Лёвенгейма приносит многообразие счетных моделей теории $\mathbf{Z F C}^{-}$:

ПРЕДЛОЖЕНИЕ 2.1. Если $X \subseteq \mathbf{H C ~ с ч е т н о , ~ т о ~ н а и ̆ д е т с я ~ с ч е т н о е ~ т р а н з и т и в - ~}$ ное множество $M \in \mathbf{H C}$, для которого $X \subseteq M u M-$ модель $\mathbf{Z F C}^{-}$.

2Б. Общее понятие конструктивности по Гёделю. Среди всех мыслимых множеств с очевидностью выделяются такие, которые допускают простое определение или явное построение с помошью простых операщий; назовем их "конструктивными". Это интуитивное понятие: оно зависит и от набора разрешенных средств определения или построения, и от выбора исходных множеств для этих определений, построений. К счастью, оказьвается, что все разумные варианты определения конструктивности приводят к очень небольшому числу действительно разных результатов. Наиболее известным и полезным является определение конструктивности по Гёделю.

Определение конструктивных множеств известно в нескольких вариантах, даюших, однако, один и тот же результат. Для нас более удобен вариант [20], прямо основанньй на восьми гёделевых операциях (даны в варианте [29]):

$$
\begin{gathered}
\mathfrak{F}_{1}(X, Y)=\{X, Y\}, \mathfrak{F}_{2}(X, Y)=X \backslash Y, \quad \mathfrak{F}_{3}(X, Y)=X \times Y, \\
\mathfrak{F}_{4}(X, Y)=\{x: \exists y(\langle x, y\rangle \in X)\}, \quad \mathfrak{F}_{5}(X, Y)=\{\langle x, y\rangle \in X: x \in y\}, \\
\mathfrak{F}_{6}(X, Y)=\{\langle x, y, z\rangle:\langle y, z, x\rangle \in X\}, \quad \mathfrak{F}_{7}(X, Y)=\{\langle x, y, z\rangle:\langle z, y, x\rangle \in X\}, \\
\mathfrak{F}_{8}(X, Y)=\{\langle x, y, z\rangle:\langle x, z, y\rangle \in X\} .
\end{gathered}
$$

\footnotetext{
${ }^{18}$ Хотя иногда, неформально, они все же называются моделями, скажем, $\mathbf{L}$ - конструктивная модель. Более точное название для структур, чьи области являютс собственными классами, - интерпретация, см. [86; 4.7 и 9.5], в данном случае интерпретация теории $\mathbf{Z F C}$ в ней самой.
} 
ОПРЕДЕЛЕНИЕ 2.2 (конструктивные множества). Определим полное упорядочение $\lessdot$ класса $T=\{0,1, \ldots, 8\} \times$ Ord $\times$ Ord так: $\langle i, \xi, \eta\rangle \lessdot\left\langle i^{\prime}, \xi^{\prime}, \eta^{\prime}\right\rangle$, когда либо $\max \{\xi, \eta\}<\max \left\{\xi^{\prime}, \eta^{\prime}\right\}$, либо $\max \{\xi, \eta\}=\max \left\{\xi^{\prime}, \eta^{\prime}\right\}$, но $\langle\xi, \eta\rangle<\left\langle\xi^{\prime}, \eta^{\prime}\right\rangle$ лексикографически, либо $\xi=\xi^{\prime}, \eta=\eta^{\prime}$ и $i<i^{\prime}$. Сушествует единственньй порядковый изоморфизм $K:\langle T ; \lessdot\rangle \stackrel{\text { на }}{\longrightarrow} \operatorname{Ord} \backslash\{0\}$. Через $K_{0}, K_{1}, K_{2}$ обозначим обратные функции, т.е. если $K(i, \xi, \eta)=\gamma$, то $K_{0}(\gamma)=i, K_{1}(\alpha)=\xi, K_{2}(\alpha)=\eta$.

Легко видеть, что при $K_{0}(\gamma)>0$ будет $K_{1}(\gamma)<\gamma$ и $K_{2}(\gamma)<\gamma$. Это позволяет определить последовательность множеств $\boldsymbol{F}_{\gamma}$ индукцией по $\gamma \in \operatorname{Ord}$, при помощи следующей схемы:

$$
\boldsymbol{F}_{\gamma}= \begin{cases}\varnothing & \text { при } \gamma=0 ; \\ \mathfrak{F}_{K_{0}(\gamma)}\left(\boldsymbol{F}_{K_{1}(\gamma)}, \boldsymbol{F}_{K_{2}(\gamma)}\right) & \text { при } \gamma>0, K_{0}(\gamma)>0 ; \\ \left\{\boldsymbol{F}_{\nu}: \nu<\gamma\right\} & \text { при } \gamma>0, K_{0}(\gamma)=0 .\end{cases}
$$

Наконец, $\mathbf{L}=\left\{\boldsymbol{F}_{\gamma}: \gamma \in \mathrm{Ord}\right\}$ есть класс всех конструктивных множеств.

ОПРЕДЕЛЕНИЕ 2.3 (относительная конструктивность). Для любого множества $X \subseteq \mathbf{L}$ пусть $\vartheta=\vartheta(X)$ - наименьший ординал такой, что $X \subseteq\left\{\boldsymbol{F}_{\xi}: \xi<\vartheta\right\}$. (Например, в случае, когда $X=a \in \mathbb{N}^{\omega}$, мы всегда имеем $\Lambda(a)=\omega$.) Переопределим $K$ в 2.2 как единственный порядковый изоморфизм $K:\langle T ; \lessdot\rangle \stackrel{\text { на }}{\longrightarrow}$ Ord $\backslash\{0, \vartheta\}$; таким образом, обратные функции $K_{0}, K_{1}, K_{2}$ определены на множестве Ord $\backslash\{0, \vartheta\}$. Определим $\boldsymbol{F}_{\xi}[X]$ индукцией по $\xi \in$ Ord в соответствии со схемой определения 2.2 , но с дополнительным специальным пунктом: $\boldsymbol{F}_{\vartheta}[X]=X$. (Заметим, что $\boldsymbol{F}_{\xi}[X]=\boldsymbol{F}_{\xi}$ при $\xi<\vartheta$, так что $X=\boldsymbol{F}_{\vartheta}[X] \subseteq\left\{\boldsymbol{F}_{\xi}[X]: \xi<\vartheta\right\}$.)

Теперь $\mathbf{L}[X]=\left\{\boldsymbol{F}_{\gamma}[X]: \gamma \in\right.$ Ord $\}$ есть класс всех множеств, конструктивных относительно $X$. Если $x, y \in \mathbf{L}[X]$, то $x<[X] y$ означает, что $x$ встречается в последовательности множеств $\boldsymbol{F}_{\gamma}[X]$ раньше $y$.

Обобшение. Пусть $s=\left\{X_{\xi}\right\}_{\xi<\zeta}$ конечная или трансфинитна последовательность множеств $X_{\xi} \subseteq \mathbf{L}(\zeta \in$ Ord). Вообше говоря, $s \nsubseteq \mathbf{L}$, но можно определить $\mathbf{L}[s]=$ $\mathbf{L}[X]$, где $X=\left\{\langle\xi, x\rangle: x \in X_{\xi}\right\} \subseteq \mathbf{L}$. Это определение работает, в частности, для классов вида $\mathbf{L}\left[a_{1}, \ldots, a_{n}\right]$, где $a_{1}, \ldots, a_{n} \subseteq \mathbf{L}$, например, $a_{1}, \ldots, a_{n} \in \mathbb{N} \omega$.

Нетрудно проверить, что класс $\mathbf{L}$ совпадает с $\mathbf{L}[X]$ для любого $X \in \mathbf{L}$. Следующая теорема получена в [20] именно в этом частном случае, но общий результат доказывается аналогично.

ТеОрема 2.4. Пусть $X \subseteq \mathbf{L}$. В классе $\mathbf{L}[X]$ выполнены все аксиомы ZFC. Класс $\mathbf{L}[X]$ содержит элемент $X$ и все ординаль и является наименьшим классом с этим свойством, в котором выполнены все аксиомы ZFC.

$<[X]$ есть полное упорядочение класса $\mathbf{L}[X]$, порядково подобное Ord.

Гёдель установил также, что аксиома выбора $\mathbf{A C}$ и обобщенная континуум-гипотеза истинны в $\mathbf{L}$ (на самом деле, и в любом классе вида $\mathbf{L}[a], a \in \mathbb{N}^{\omega}$, но континуум-гипотеза уже не обязательно верна в $\mathbf{L}[X]$ для произвольного $X)$.

Следующий простой факт будет использован ниже.

ПРЕДЛОЖЕНИЕ 2.5. Пусть М - транзитивное множество, замкнутое относительно гёделевьх операций, и $а \in \mathfrak{M} \cap \mathbb{N}^{\omega}$. Предположим также, что для любого ординала $\gamma \in \mathfrak{M}$ ограниченная последовательность $\left\{\boldsymbol{F}_{\xi}[a]\right\}_{\xi<\gamma}$ принадле-

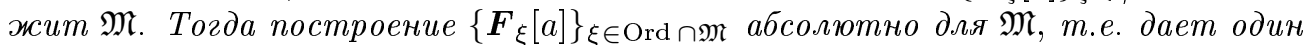
и тот же результат в $\mathfrak{M}$ и в универсуме всех множеств. 
2В. Конструктивность в области бэровского пространства. Пусть $a \in \mathbb{N} \omega$. Положим, для $\xi \in \operatorname{Ord}, \boldsymbol{f}_{\xi}[a]=\boldsymbol{F}_{\xi}[a]$, если $\boldsymbol{F}_{\xi}[a] \in \mathbb{N}^{\omega}$, а иначе определим $\boldsymbol{f}_{\xi}[a]=\mathbf{0}$, т.е. функция $\mathbf{0}(n)=0, \forall n$, так что $\boldsymbol{f}_{\xi}[a]$ всегда принадлежит $\mathbb{N}^{\omega}$.

Через $\omega_{1}^{\mathbf{L}[a]}$ обозначим первьй несчетный кардинал в классе $\mathbf{L}[a]$.

Через $\prec_{a}$ обозначим порядок $<[a]$, ограниченньй на $\mathbf{L}[a] \cap \mathbb{N}^{\omega}$.

ТЕОРема 2.6. Виполняются следующие утверждения.

(i) Ecлu $a \in \mathbb{N}^{\omega}$, mo $\mathbf{L}[a] \cap \mathbb{N}^{\omega}=\left\{f_{\xi}[a]: \xi<\omega_{1}\right\}=\left\{f_{\xi}[a]: \xi<\omega_{1}^{\mathrm{L}[a]}\right\} u$ отношение $\prec_{а}$ вполне упорядочивает $\mathbf{L}[a] \cap \mathbb{N}^{\omega}$ nо типу $\omega_{1}^{\mathrm{L}[a]}$.

(ii) Найдутся $\Sigma_{1}^{1}$-формула $\varphi$ и $\Pi_{1}^{1}$-формула $\boldsymbol{\psi}$ такие, что для любьх $w \in \mathbf{W O}$ $u a, x \in \mathbb{N}^{\omega}$ выполнено $x=\boldsymbol{f}_{|w|}[a] \Longleftrightarrow \varphi(w, a, x) \Longleftrightarrow \psi(w, a, x)$.

(iii) Множество $\mathbf{L}[a] \cap \mathbb{N}^{\omega}$ и отношение $\prec_{a}$ принадлежат классу $\Sigma_{2}^{1}(a)$.

(iv) Eсли $a, p \in \mathbb{N}^{\omega}, P \subseteq \mathbb{N}^{\omega} \times \mathbb{N}^{\omega}$ - множество класса $\Delta_{2}^{1}(p)$,

$X=\left\{x \in \mathbb{N}^{\omega}:\right.$ множество $P_{x}(a)=\{y \in \mathbf{L}[a]:\langle x, y\rangle \in P\}$ непусто $\}$,

$a$ для $x \in X$ через $y_{x}$ обозначен $\prec_{а}$-наименьший әлемент $P_{x}$, то $X u$ $P^{\prime}=\left\{\left\langle x, y_{x}\right\rangle: x \in X\right\}$ принадлежст $\Sigma_{2}^{1}(a, p)$. Если известно, что $X$ есть $\Delta_{2}^{1}(a, p)$, то $P^{\prime}$ также является $\Delta_{2}^{1}(a, p)$-множеством.

ДокАЗАТЕльство (набросок). (i) Любое $x \in \mathbb{N}^{\omega}$ из $\mathbf{L}[a]$ появляется в построении $\mathbf{L}[a]$ до шага $\omega_{1}$. Это важнейший шаг в гёделевом доказательстве континуум-гипотезы в конструктивном универсуме $\mathbf{L}$ в работе [20], основанный на методе Скулема-Лёвенгейма. (Обобщение на $\mathbf{L}[a], a \in \mathbb{N} \omega$, не вносит никаких новых моментов.) Отсюда следует равенство $\mathbf{L}[a] \cap \mathbb{N}^{\omega}=\left\{\boldsymbol{f}_{\xi}[a]: \xi<\omega_{1}\right\}$, а также то, что длина $\prec_{a}$ не превосходит $\omega_{1}$. Почему $\omega_{1}$ можно заменить на $\omega_{1}^{\mathrm{L}[a]}$ ? Дело в том, что индуктивная конструкция множеств $\boldsymbol{F}_{\xi}[a]$ абсолютна для $\mathbf{L}[a]$, т.е. может быть проведена в $\mathbf{L}[a]$ с тем же конечным результатом. Однако в $\mathbf{L}[a]$ все точки $\mathbb{N}^{\omega}$ появляются до шага $\omega_{1}^{\mathbf{L}[a]}$ !

(ii) Идея доказательства состоит в том, чтобы кодировать всю гёделеву конструкцию до шага $\omega_{1}$ (где мы имеем, очевидно, только не более чем счетные множества) посредством точек $\mathbb{N} \omega$. Это, в принципе, аналогично кодировке счетных ординалов или борелевских множеств в п. 1Д, но технические детали много более сложны; в деталях все рассуждение проведено П. С. Новиковым [78] и Аддисоном [1], [2] (в несколько отличающихся вариантах и в предположении $\mathbf{V}=\mathbf{L}$ ). Мы дадим набросок еще одного варианта доказательства, чтобы дать возможность читателю составить представление о механизмах таких рассуждений без обращения к трудночитаемым первоисточникам.

Мы будем рассматривать структуры вида $\langle\mathbb{N} ; \varepsilon\rangle$, где бинарное отношение $\varepsilon \subseteq \mathbb{N}^{2}$ понимается как "принадлежность" в $\langle\mathbb{N} ; \varepsilon\rangle$. Обозначим через $E$ множество всех $\varepsilon \subseteq \mathbb{N}^{2}$, удовлетворяюших следуюшим трем условиям:

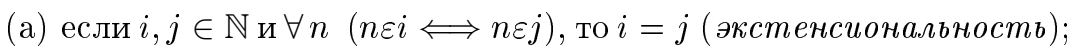

(b) все аксиомы $\mathbf{Z F C}{ }^{-}$(т.е. ZFC без аксиомы степени, п. $2 \mathrm{~A}$ ) истинны в $\langle\mathbb{N} ; \varepsilon\rangle$;

(c) ординальньй ряд $\operatorname{Ord}^{\varepsilon}=\{n \in \mathbb{N}$ : в $\langle\mathbb{N} ; \varepsilon\rangle$ истинно, что $n$-ординал $\}$ модели $\langle\mathbb{N} ; \varepsilon\rangle$ имеет начальный сегмент, подобный (порядково изоморфный) ординалу $\omega+1$ (иными словами, $\langle\mathbb{N} ; \varepsilon\rangle$ есть $\omega$-модель). 
В этом случае для каждого $n \in \mathbb{N}$ существует единственньй элемент $n^{\varepsilon} \in \mathbb{N}$, соответствующий $n$ в $\langle\mathbb{N} ; \varepsilon\rangle$, а также существует единственный элемент $\omega^{\varepsilon} \in \mathbb{N}$, соответствующий $\omega$ в $\langle\mathbb{N} ; \varepsilon\rangle$, и $\left\{n^{\varepsilon}: n \in \mathbb{N}\right\}$ - множество всех $\varepsilon$-элементов $\omega^{\varepsilon}$.

Предположим, что $x \in \mathbb{N}^{\omega}$. Может найтись (единственньй, если существует) элемент $k \in \mathbb{N}$ такой, что в $\langle\mathbb{N} ; \varepsilon\rangle$ истинно: " $k \in \mathbb{N}^{\omega}$ и $k\left(n^{\varepsilon}\right)=m^{\varepsilon}$ " всякий раз, когда $x(n)=m$. Такой элемент $k$ будет обозначаться через $x^{\varepsilon}$. Понятно, что $x^{\varepsilon}$ сушествует лишь для не более чем счетного множества точек $x \in \mathbb{N}^{\omega}$.

Возьмем в качестве формулы $\varphi(w, a, x)$ для доказательства (ii) формулу:

$\exists \varepsilon \in E\left(a^{\varepsilon}, w^{\varepsilon}, x^{\varepsilon}\right.$ существуют в $\langle\mathbb{N} ; \varepsilon\rangle \wedge$ в $\langle\mathbb{N} ; \varepsilon\rangle$ истинно $\left.x^{\varepsilon}=\boldsymbol{f}_{\left|w^{\varepsilon}\right|}\left[a^{\varepsilon}\right]\right)$.

Каждый из двух конъюнктивных членов формулы в скобках выражается арифметической формулой, так что в результате действительно получается $\Sigma_{1}^{1}$-формула. (Мы отвлекаемся от того, что, скажем, $\varepsilon$ нужно еще кодировать точкой $\mathbb{N} \omega$, поскольку здесь нет никаких проблем.)

Остается проверить, что так определенная формула $\varphi$ удовлетворяет (ii).

Предположим, что $w \in \mathbf{W O}, a, x \in \mathbb{N}^{\omega}$ и имеет место $\varphi(w, a, x)$, т.е. имеется $\varepsilon \in E$ такое, что $a^{\varepsilon}, w^{\varepsilon}, x^{\varepsilon}$ сушествуют в $\langle\mathbb{N} ; \varepsilon\rangle$ и $x^{\varepsilon}=\boldsymbol{f}_{\left|w^{\varepsilon}\right|}\left[a^{\varepsilon}\right]$ истинно в $\langle\mathbb{N} ; \varepsilon\rangle$. Тогда ординальньй ряд $\operatorname{Ord}^{\langle\mathbb{N} ; \varepsilon\rangle}$ имеет начальньй сегмент, подобньй $\xi=|w|$, следовательно, имеет начальный сегмент, подобный $\xi+\omega+1$ (а также начальные сегменты, подобные $\xi+\xi, \xi \times \xi$ и т. п.). Покажем, что вследствие этого модель $\langle\mathbb{N} ; \varepsilon\rangle$ содержит в себе хороший кусок $\in$-структуры обычных множеств.

Напомним, что rk $x \in$ Ord - теоретико-множественный ранг, см. п. 2А. Пусть $M$ есть множество всех $m$ таких, что в $\langle\mathbb{N} ; \varepsilon\rangle$ истинно: "rk $x<\left|w^{\varepsilon}\right|+\omega^{\varepsilon}$ ". Легко видеть, что каждое $m \in M$ принадлежит фундированной части $\mathbf{W} \mathbf{F}_{\varepsilon}$ модели $\langle\mathbb{N} ; \varepsilon\rangle$, которая состоит из всех $m$ таких, что $\varepsilon$-транзитивное замыкание $\mathrm{TC}_{\varepsilon}(m)$ не содержит бесконечно $\varepsilon$-убьваюших цепочек. ( $\mathrm{TC}_{\varepsilon}(m)$ есть наименьшее множество $T \subseteq \mathbb{N}$ такое, что $m \in T$ и $i \varepsilon j \in T \Longrightarrow i \in T$.) Следовательно, мы можем определить настояшее множество $S(m)$ для каждого $m \in M$ в соответствии с равенством $S(m)=\{S(n): n \varepsilon m\}$.

УТВЕРЖДЕНИЕ 2.7. Множество $M$ содержит $a^{\varepsilon}, w^{\varepsilon}, x^{\varepsilon}$, множество $\mathbf{S}=$ $\{S(n): n \in M\}$ транзитивно, а отображсение $m \mapsto S(m)$ есть изоморфизм $\langle M ; \varepsilon\rangle$ на $\langle\mathbf{S} ; \in\rangle$, причем $S\left(w^{\varepsilon}\right)=w, S\left(x^{\varepsilon}\right)=x, S\left(a^{\varepsilon}\right)=a$.

Заметим, что структура $\langle M ; \varepsilon\rangle$, с точки зрения модели $\langle\mathbb{N} ; \varepsilon\rangle$, есть множество вида $\{z: \operatorname{rk} z<\xi+\omega\}$, где $\xi=|w|$, а каждое такое множество, очевидно, удовлетворяет требованиям, предъявляемым к $\mathfrak{M}$ предложением 2.5. Поэтому формула $x^{\varepsilon}=f_{\left|w^{\varepsilon}\right|}\left[a^{\varepsilon}\right]$ истинна в $\langle M ; \varepsilon\rangle$, будучи по предположению истинной в $\langle\mathbb{N} ; \varepsilon\rangle$. Следовательно, согласно $2.7, x=\boldsymbol{f}_{|w|}[a]$ истинно в $\langle\mathbf{S} ; \in\rangle$. Опять согласно предложению 2.5 , формула $x=\boldsymbol{f}_{|w|}[a]$ выполнена и в универсуме всех множеств. Итак, $\varphi(w, a, x)$ влечет $x=f_{|w|}[a]$.

Обратно, предположим, что $x=\boldsymbol{f}_{|w|}[a]$. Согласно предложению 2.1 , найдется счетное транзитивное множество $\mathfrak{M} \subseteq \mathbf{H C}$, содержашее $w, a, x$ и являюшееся моделью $\mathbf{Z F C}{ }^{-}$. Тогда формула $x=\boldsymbol{f}_{|w|}[a]$ истинна в $\langle\mathfrak{M} ; \in\rangle$. Рассмотрим такое $\varepsilon \subseteq \mathbb{N}^{2}$, что модель $\langle\mathbb{N} ; \varepsilon\rangle$ изоморфна $\langle\mathfrak{M} ; \in\rangle$. Понятно, что $\varepsilon \in E$ и (единственншй) изоморфизм переводит $w, a, x$ в, соответственно, $w^{\varepsilon}, a^{\varepsilon}, x^{\varepsilon}$, которые существуют в $\langle\mathbb{N} ; \varepsilon\rangle$, так что формула $x^{\varepsilon}=\boldsymbol{f}_{\left|w^{\varepsilon}\right|}\left[a^{\varepsilon}\right]$ истинна в $\langle\mathbb{N} ; \varepsilon\rangle$. Это означает, что выполнено $\varphi(w, a, x)$.

А в качестве $\Pi_{1}^{1}$-формулы $\psi$ можно взять следуюшую:

$$
\forall \varepsilon \in E\left(a^{\varepsilon}, w^{\varepsilon}, x^{\varepsilon} \text { сушествуют в }\langle\mathbb{N} ; \varepsilon\rangle \Longrightarrow \text { в }\langle\mathbb{N} ; \varepsilon\rangle \text { истинно } x^{\varepsilon}=\boldsymbol{f}_{\left|w^{\varepsilon}\right|}\left[a^{\varepsilon}\right]\right) \text {. }
$$


(iii) Из (i) следует, что $x \in \mathbf{L}[a] \cap \mathbb{N}^{\omega} \Longleftrightarrow \exists w\left(w \in \mathbf{W O} \wedge x=f_{|w|}[a]\right)$. Заменим равенство $x=f_{|w|}[a] \quad \Sigma_{1}^{1}$-формулой $\varphi(w, a, x)$ из (ii) и воспользуемся тем, что $\mathbf{W O} \in \Pi_{1}^{1}$ (предложение 1.11). Что же касается порядка $\prec_{a}$, то по определению $x \prec_{a} y$ равносильно тому, что

$$
\exists \eta<\omega_{1}(y=\boldsymbol{f}_{\eta}[a] \wedge \underbrace{\forall \xi<\eta\left(y \neq \boldsymbol{f}_{\xi}[a]\right)}_{\vartheta(a, y, \eta)} \wedge \exists \xi<\eta\left(x=\boldsymbol{f}_{\xi}[a]\right)) .
$$

Теперь заметим, что, для любого $w \in \mathbf{W O}$,

$$
\vartheta(a, y,|w|) \Longleftrightarrow \forall k\left(y \neq \boldsymbol{f}_{w \uparrow_{k}}[a]\right)
$$

(см. пример 1.10.1). Это позволяет привести формулу $(*)$ к искомому $\Sigma_{2}^{1}$-виду (от аргументов $x, y, a)$ при помоши (ii) и предложения 1.11. Как видно, ключевым моментом является преврашение квантора $\forall \xi<\eta$ подформулы $\vartheta$ в квантор над $\mathbb{N}$, которьй не влияет на класс формулы.

(iv) Прежде всего, $x \in X \Longleftrightarrow \exists y \quad\left(y \in \mathbf{L}[a] \cap \mathbb{N}^{\omega} \wedge\langle x, y\rangle \in P\right)$, откуда в силу первого утверждения (iii) следует $X \in \Sigma_{2}^{1}(a, p)$. Далее, $\langle x, y\rangle \in P^{\prime}$ равносильно

$$
\langle x, y\rangle \in P \wedge \exists \eta<\omega_{1}(y=\boldsymbol{f}_{\eta}[a] \wedge \underbrace{\forall \xi<\eta \exists z\left(z=\boldsymbol{f}_{\xi}[a] \wedge\langle x, z\rangle \notin P\right)}_{\tau(a, x, \eta)}),
$$

где формула $\tau(a, x, \eta)$ выражает тот факт, что $\left\langle x, f_{\xi}[a]\right\rangle \notin P$ для любого $\xi<\eta$. Приведение всей выделенной формулы к $\Sigma_{2}^{1}$-виду осушествляется теми же рассуждениями, что и выше. Наконец, $\langle x, y\rangle \in P^{\prime} \Longleftrightarrow x \in X \wedge \forall z \neq y\left(\langle x, z\rangle \notin P^{\prime}\right)$, откуда следует последнее утверждение (iv).

2Г. Абсолютность. Это понятие прямо не связано с конструктивностью, однако необходимость в нем возникает сразу, как только мы узнаем, что универсум $\mathbf{V}$ всех множеств имеет подклассы, например, классы вида $\mathbf{L}[X]$, которые сами удовлетворяют аксиомам $\mathbf{Z F C}$ и поэтому могут рассматриваться как полноценные теоретико-множественные универсумы.

Предположим, что $\mathfrak{M}$ - транзитивное множество или класс (например, класс вида $\mathbf{L}[X]$ ), который удовлетворяет аксиомам теории $\mathbf{Z F C}$ (или хотя бы некоторой значительной части $\mathbf{Z F C}$, как, скажем, $\mathbf{Z F C} \mathbf{C}^{-}$). Пусть $\Phi$ - замкнутая формула с параметрами из $\mathfrak{M}$. Тогда можно ставить вопрос об истинности или ложности $\Phi$ не только в универсуме $\mathbf{V}$, но и в $\mathfrak{M}$, понимая под этим истинность или ложность (в $\mathbf{V}$ ) той формулы, которая получается из $\Phi$ релятивизацией $\kappa \mathfrak{M}$, т.е. заменой всех кванторов $\exists x$ и $\forall x$ на $\exists x \in \mathfrak{M}$ и $\forall x \in \mathfrak{M}$. Если $\Phi$ одновременно истинна или одновременно ложна в $\mathfrak{M}$ и в универсуме $\mathbf{V}$ всех множеств, то говорят, что $\Phi$ абсолютна для $\mathfrak{M}$.

В сушности, мы уже встречались с понятием абсолютности в простой ситуации, когда абсолютность рассматриваемых формул или определяемых ими конструкций достаточно очевидна (предложение 2.5 и его использование в доказательстве теоремы 2.6(ii)). Следующая теорема ценна тем, что позволяет установить абсолютность формулы на основании анализа только ее синтаксического строения, без учета ее математического содержания. 
ТЕОРема 2.8 (теорема абсолютности). Предположим, что $\mathfrak{M}$ - транзитивное множество или класс, в котором истинны все аксиомы $\mathbf{Z F C}^{-}$. Тогда

(i) каждая $\Sigma_{1}^{1}$-формула с параметрами из $\mathfrak{M} \cap \mathbb{N}^{\omega}$ абсолютна для $\mathfrak{M}$;

(ii) если $\omega_{1} \subseteq \mathfrak{M}$, то то же верно для $\Sigma_{2}^{1}$-формул с параметрами из $\mathfrak{M} \cap \mathbb{N}^{\omega}$.

ДоказАТельство. Справедливость (i) (теорема Мостовского) основана на том, что, по теореме просеивания $1.6, \neg \Phi$ равносильно полной упорядоченности некоторого множества $Q \in \mathfrak{M}, Q \subseteq \mathbb{Q}$, которое зависит от $\Phi$, но не от $\mathfrak{M}$. Доказательство (iі) (теорема абсолютности Шёнфилда), впервые данное в [85], несколько более сложно, на русском языке см. [35; с. 305]. В форме упражнений с набросками доказательств оба утверждени даны в [86; упр. 12 к гл. 9].

ЗАмечАнИЕ 2.9. Утверждение (i) теоремы 2.8 переносится также на $\Pi_{1}^{1}$-формулы, а (iі) на $\Pi_{2}^{1}$-формулы. Более того, односторонняя абсолютность распространяется на следующий уровень, например, если $\omega_{1} \subseteq \mathfrak{M}$, то каждая $\Sigma_{3}^{1}$-формула с параметрами из $\mathfrak{M} \cap \mathbb{N}^{\omega}$, истинная в $\mathfrak{M}$, истинна и в универсуме $\mathbf{V}$ всех множеств, а для $\Pi_{3}^{1}$-формул верно обратное.

Предложение 1.11 (как и другие подобные результаты об определимости) ведет к многочисленным приложениям теоремы абсолютности. Например, из 1.11(i) следует, что $(\mathbf{B C})^{\mathfrak{M}}=\mathbf{B C} \cap \mathfrak{M}$, т.е. любое $c \in \mathbb{N}^{\omega} \cap \mathfrak{M}$ принадлежит $\mathbf{B C}$ тогда и только тогда, ког да в $\mathfrak{M}$ истинно, что $c \in \mathbf{B C}$. Из 1.11(iv) следует, что, при $c \in \mathbf{B C} \cap \mathfrak{M}$, множество $\left(\boldsymbol{B}_{c}\right)^{\mathfrak{M}}$, т.е. $\boldsymbol{B}_{c}$, определенное в $\mathfrak{M}$, совпадает с $\boldsymbol{B}_{c} \cap \mathfrak{M}$. А если $\omega_{1} \subseteq \mathfrak{M}$ и $p, q \in \mathbf{B C} \cap$ $\mathfrak{M}$, то опять из 1.11(iv) следует, что $\boldsymbol{B}_{p} \subseteq \boldsymbol{B}_{q}$ равносильно $\boldsymbol{B}_{p} \cap \mathfrak{M} \subseteq \boldsymbol{B}_{q} \cap \mathfrak{M}$, а $\boldsymbol{B}_{p}=\boldsymbol{B}_{q}$ равносильно $\boldsymbol{B}_{p} \cap \mathfrak{M}=\boldsymbol{B}_{q} \cap \mathfrak{M}$.

Теорема 2.8 также позволяет получать такие эффективные формы классических теорем дескриптивной теории множеств, прямое доказательство которых потребовало бы нудного копания в деталях. Примером является

Tеорема 2.10 (эффективная теорема Суслина). Если $a \in \mathbb{N}^{\omega} u X \subseteq \mathbb{N}^{\omega}-$ множество класса $\Delta_{1}^{1}(a)$, то найдется код $c \in \mathbf{B C} \cap \mathbf{L}[a]$, для которого $X=\boldsymbol{B}_{c}$.

ДокАЗАТЕльство. Рассмотрим $\Sigma_{1}^{1}$-формулу $\varphi$ и $\Pi_{1}^{1}$-формулу $\psi$ такие, что $X=$ $\{x: \varphi(a, x)\}=\{x: \psi(a, x)\}$. Эквивалентность $\forall x \quad(\varphi(a, x) \Longleftrightarrow \psi(a, x))$ выражается $\Pi_{2}^{1}$-формулой с параметром $a$, поэтому она верна и в $\mathbf{L}[a]$, так что эквивалентные формулы $\varphi$ и $\psi$ определяют $\boldsymbol{\Delta}_{1}^{1}$-множество в $\mathbf{L}[a]$. Значит, по классической теореме Суслина (см. теорему 1.8), найдется $c \in \mathbf{L}[a] \cap \mathbb{N}^{\omega}$ такое, что в $\mathbf{L}[a]$ истинно: $c \in \mathbf{B C}$ и $\forall x \quad\left(x \in \boldsymbol{B}_{c} \Longleftrightarrow \varphi(a, x) \Longleftrightarrow \psi(a, x)\right)$. Однако эта формула - также $\Pi_{2}^{1}$ с параметрами $a, c$ (предложение 1.11(iv)), поэтому снова по теореме 2.8 мы имеем $c \in \mathbf{B C}$ и $X=\boldsymbol{B}_{c}$ в универсуме $\mathbf{V}$ всех множеств.

Исторические и библиографические замечания. Об исследованиях, приведших к возникновению той картины теоретико-множественного универсума, которая вкратце обозначена в п. 2А, и к теории ZFC, см., например, [19].

Теорема абсолютности 2.8(ii) была доказана Шёнфилдом [85].

Kласс L всех конструктивных множеств построен Гёделем [20]. Специфичиеские вопросы конструктивного построения в области бэровского пространства, включая дескриптивную определимость гёделевой последовательности, ограниченной счетными ординалами, и соответствуюшее полное упорядочение континуума, были рассмотрены П. С. Новиковым [78] и вслед за ним Аддисоном [1], [2]. Относительная конструктивность (т.е. классы вида $\mathbf{L}[x]$ ) стала рассматриваться уже в $60-е$ годы, в частности 
в связи с развитием форсинга. Ссылки по конструктивности на русском языке, включая ее приложения в дескриптивной теории множеств, даны в [29], [35] и [11; гл. 5].

Систематическое изложение свойств важного множества всех конструктивных точек (т.е. множества $\mathbf{L}[a] \cap \mathbb{N}^{\omega}$ ) приводится в работе авторов, которая сейчас готовится к печати в томе “Трудов Математического института им. В. А. Стеклова", посвященном Л. В. Келдыш.

\section{$\S$ 3. Резольвенты классических проблем: первая часть}

Проблемы о свойстве совершенного ядра для $\Pi_{1}^{1}$-множеств и об измеримости и свойстве Бэра для множеств второго проективного уровня оказываются тесно связанными с особыми свойствами некоторых специальных множеств в смысле, близком к тому, что Лузин называл резольвентами.

Если $\mathrm{P}(\mathbf{K})$ - проблема существования в данном проективном классе $\mathbf{K}$ множества с определенньм свойством (например, неизмеримого или не обладающего свойством Бэра), то обычно не составляет труда определить, при помоши язька аналитических формул, такое проективное множество $X$, что проблема $\mathrm{P}(\mathbf{K})$ имеет положительное решение, если и только если $X$ непусто. Такие множества $X$ Лузин [54] назьвал резольвентами (résolvantes) исходных проблем. В более широком смысле, резольвентой проблемы $\mathrm{P}(\mathbf{K})$ мы будем называть определенное свойство $\boldsymbol{p}$ определенного множества $X$, равносильное положительному решению этой проблемы. (По Лузину, $\boldsymbol{p}$ есть непустота $X$.)

В этом параграфе мы начинаем изложение исследований по резольвентам классических проблем о свойствах регулярности, изображенных на приведенной во введении диаграмме, которые раскрыли сушество этих проблем и позволили установить связи между ними.

3А. Коэновские и случайные точки. Резольвенты, о которых идет речь в главных результатах (теорема 3.3 и следствие 3.4 ниже), формулируются в терминах свойств множеств вида $\mathbf{L}[a] \cap \mathbb{N}^{\omega}, a \in \mathbb{N}^{\omega}$, а также множеств, которые вводятся следуюшим определением.

ОПРЕДЕЛЕНИЕ 3.1. Пусть $\mathfrak{M}$ - транзитивная модель ZFC, например, класс вида $\mathbf{L}[X]$. Назовем точку $x \in \mathbb{N}^{\omega}$ коэновской над $\mathfrak{M}$, символически $x \in \mathbf{C o h} \mathfrak{M}$, если $x \notin \boldsymbol{B}_{c}$ всякий раз, когда $c \in \mathfrak{M} \cap \mathbf{B C}$ и $\boldsymbol{B}_{c}-$ тощее множество в $\mathbb{N}^{\omega}$.

Пусть, дополнительно, $\mu$ есть борелевская мера на $\mathbb{N}^{\omega}$. Точка $x \in \mathbb{N}^{\omega}$ назьвается $\mu$-случайной над $\mathfrak{M}$, символически $x \in \boldsymbol{R a n d}_{\mu} \mathfrak{M}$, если $x \notin \boldsymbol{B}_{c}$ всякий раз, когда $c \in \mathfrak{M} \cap \mathbf{B C}$ и $\mu\left(\boldsymbol{B}_{c}\right)=0$. Положим $\mathbf{R a n d} \mathfrak{M}=\operatorname{Rand}_{\boldsymbol{\lambda}} \mathfrak{M}^{19}$

Таким образом, коэновские точки (над $\mathfrak{M})$ - это те, которые избегают любое тощее борелевское множество с кодом из $\mathfrak{M}$. Соответственно, случайные точки - это те, которые избегают множества нулевой меры. Коэновские и случайные точки допускают удобную характеризацию в терминах форсинга; об этом см. в п. 4В.

Заметим, что Rand $\mathfrak{M} \subseteq 2^{\omega}$, поскольку $\boldsymbol{\lambda}\left(\mathbb{N}^{\omega} \backslash 2^{\omega}\right)=0$.

Следуюшая лемма понадобится нам ниже. Ее утверждения (1), (2) показьвают, что, из-за определенной однородности меры и категории, непустота множеств Coh $\mathfrak{M}$ и Rand $\mathfrak{M}$ влечет их плотность в достаточно сильном смысле.

\footnotetext{
${ }^{19}$ Напомним, что мера $\boldsymbol{\lambda}$ на $2^{\omega}$ введена в п. 1Б, а кодировка борелевских множеств, вместе с определениями $\mathbf{B C}$ и $\boldsymbol{B}_{c},-$ в п. 1Д. Тощие множества - это множества 1-й категории, а котощие - дополнительные к ним множества.
} 
Лемма 3.2. Пусть М - транзитивная модель ZFC. Тогда

(1) если Rand $\mathfrak{M} \neq \varnothing$, то любое борелевское множество ненулевой $\boldsymbol{\lambda}$-мерь с кодом из $\mathfrak{M}$ непусто пересекает Rand $\mathfrak{M}$;

(2) если $\operatorname{Coh} \mathfrak{M} \neq \varnothing$, то любое не тощее борелевское мнохсество с кодом из $\mathfrak{M}$ непусто пересекает $\operatorname{Coh} \mathfrak{M}$;

(3) условия Rand $\mathfrak{M} \neq \varnothing u \boldsymbol{\lambda}($ Rand $\mathfrak{M})=1$ не зависят от выббора мерь в следующем смысле: если $\mu$-борелевская конечная мера на $\mathbb{N}^{\omega}$ и ее код $\operatorname{Cod}(\mu)$ (т.е. функция $\left.\operatorname{Cod}(\mu)(n)=\mu\left(\mathscr{N}_{s_{n}}\right)\right)$ принадлежстт $\mathfrak{M}$, то

$$
\begin{gathered}
\operatorname{Rand} \mathfrak{M} \neq \varnothing \Longleftrightarrow \operatorname{Rand}_{\mu} \mathfrak{M} \neq \varnothing \quad u \\
\lambda(\operatorname{Rand} \mathfrak{M})=1 \Longleftrightarrow \mu\left(\operatorname{Rand}_{\mu} \mathfrak{M}\right)=\mu\left(\mathbb{N}^{\omega}\right) .
\end{gathered}
$$

ДокаЗАТельство. (1) Для случая меры пусть $c \in \mathbf{B C} \cap \mathfrak{M}$ и $m=\boldsymbol{\lambda}\left(\boldsymbol{B}_{c}\right)>0$. Рассуждение из доказательства леммы 1.3 приносит нам борелевские множества $X \subseteq 2^{\omega}$ и $Y \subseteq \boldsymbol{B}_{c}$ с $\boldsymbol{\lambda}(X)=1$ и $\boldsymbol{\lambda}(Y)=m$ и борелевский изоморфизм $F: X \stackrel{\text { на }}{\longrightarrow} Y$, сохраняющий $\boldsymbol{\lambda}$ с коэффициентом $\varkappa=\frac{m}{M}$ в том смысле, что $\boldsymbol{\lambda}\left(F^{\prime \prime} B\right)=\varkappa \boldsymbol{\lambda}(B)$ для любого борелевского $B \subseteq X$. Более того, раз $c \in \mathfrak{M}$, то, по теореме абсолютности 2.8 , множества $X, Y, F$ могут быть подобраны с кодами в $\mathfrak{M}$, в частности, тогда $F$ переводит борелевские $\lambda$-нулевые множества $B \subseteq X$ с кодами из $\mathfrak{M}$ снова в борелевские $\lambda$-нулевые множества $B \subseteq Y$ с кодами из $\mathfrak{M}$, и обратно. Поэтому если $x \in \mathbf{R a n d} \mathfrak{M}$, то $F(x) \in \mathbf{R a n d} \mathfrak{M} \cap \boldsymbol{B}_{c}$.

(2) Рассуждаем аналогично, используя то, что если $A \subseteq \mathbb{N}^{\omega}$ - не тошее борелевское множество, то найдутся бэровский интервал $\mathscr{N}_{s}$ и $\mathbf{G}_{\delta}$-множество $Y \subseteq \mathscr{N}_{s} \cap A$, плотное в $\mathscr{N}_{s}$. Как легко видеть, множество $Y$ гомеоморфно $\mathbb{N}^{\omega}$ и т. д.

(3) следует из леммы 1.3 подобно (1).

\section{3Б. Главные результаты о резольвентах свойств регулярности.}

ТеОрема 3.3. Для любой точки $a \in \mathbb{N}^{\omega}$ справедливы әквивалентности:

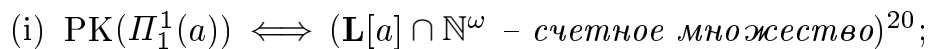

(ii) $\operatorname{LM}\left(\Sigma_{2}^{1}(a)\right) \Longleftrightarrow(\mathbf{R a n d} \mathrm{L}[a]-$ множество полной $\boldsymbol{\lambda}$-мерьи);

(iii) $\operatorname{BP}\left(\Sigma_{2}^{1}(a)\right) \Longleftrightarrow(\mathbf{C o h} \mathbf{L}[a]-$ котошее множество);

(iv) $\operatorname{LM}\left(\Delta_{2}^{1}(a)\right) \Longleftrightarrow(\operatorname{Rand} \mathbf{L}[a] \neq \varnothing)$;

(v) $\mathrm{BP}\left(\Delta_{2}^{1}(a)\right) \Longleftrightarrow(\operatorname{Coh} \mathbf{L}[a] \neq \varnothing)$.

СлЕДСТВИЕ 3.4 .

(I) $\operatorname{PK}\left(\mathbf{\Pi}_{1}^{1}\right) \Longleftrightarrow \forall a \in \mathbb{N}^{\omega} \quad\left(\mathbf{L}[a] \cap \mathbb{N}^{\omega}\right.$ счетно);

(II) $\operatorname{LM}\left(\boldsymbol{\Sigma}_{2}^{1}\right) \Longleftrightarrow \forall a \in \mathbb{N}^{\omega}$ (Rand $\mathbf{L}[a]-$ множество полной $\boldsymbol{\lambda}$-мерь);

(III) $\operatorname{BP}\left(\Sigma_{2}^{1}\right) \Longleftrightarrow \forall a \in \mathbb{N}^{\omega}$ (Coh L $[a]-$ котощее множество);

(IV) $\operatorname{LM}\left(\boldsymbol{\Delta}_{2}^{1}\right) \Longleftrightarrow \forall a \in \mathbb{N}^{\omega} \quad($ Rand $\mathrm{L}[a] \neq \varnothing)$

(V) $\operatorname{BP}\left(\boldsymbol{\Delta}_{2}^{1}\right) \Longleftrightarrow \forall a \in \mathbb{N}^{\omega} \quad(\operatorname{Coh} \mathbf{L}[a] \neq \varnothing)$.

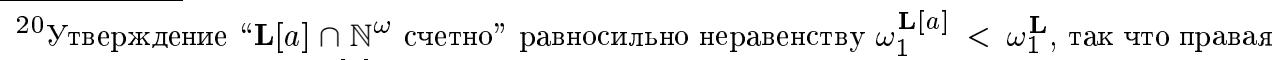
часть в (i) равносильна $\omega_{1}^{\mathbf{L}[a]}<\omega_{1}^{\mathbf{L}}-$ и в такой форме чаще встречается в литературе.
} 
ДокАЗАТЕльСТво. $\boldsymbol{\Pi}_{1}^{1}=\bigcup_{a \in \mathbb{N} \omega} \Pi_{1}^{1}(a)$, и аналогично для классов $\boldsymbol{\Sigma}_{2}^{1}, \boldsymbol{\Delta}_{2}^{1}$.

Указанные в правых частях этих эквивалентностей свойства множеств $\mathbf{L}[a] \cap \mathbb{N} \omega$, $\operatorname{Rand} \mathrm{L}[a]$, CohL $[a]$ могут рассматриваться как резольвенты проблем в левых частях эквивалентностей, в том смысле, как мы говорили вьше. Теорема 3.3 и следствие 3.4 сами по себе не решают ни одну из этих проблем (в левых частях эквивалентностей), например, они ничего не говорят о том, верна ли на самом деле гипотеза $\mathrm{PK}\left(\boldsymbol{\Pi}_{1}^{1}\right)$. Однако эти проблемы приводятся к единообразной и интуитивно более ясной форме. Тем самьм, соотношения между проблемами становятся в целом значительно более прозрачными. В частности, мы немедленно получаем импликации на диаграмме из введения, исходящие из бокса $\operatorname{PK}\left(\boldsymbol{\Pi}_{1}^{1}\right)$ :

С ЛЕДСТВИЕ 3.5 (Любецкий [65], [66], [68]). $\operatorname{PK}\left(\boldsymbol{\Pi}_{1}^{1}\right)$ влечет $\operatorname{LM}\left(\boldsymbol{\Sigma}_{2}^{1}\right)$ u $\operatorname{BP}\left(\boldsymbol{\Sigma}_{2}^{1}\right)$.

ДокАЗАТЕльство. Из счетности $\mathbf{L}[a] \cap \mathbb{N}^{\omega}$ следует, что $\operatorname{Rand} \mathbf{L}[a]-$ дополнение счетного объединения множеств меры 0 , поэтому $\boldsymbol{\lambda}(\operatorname{Rand} \mathbf{L}[a])=1$, a Coh L $[a]-$ котощее множество по аналогичным соображениям.

В этом параграфе доказьваются импликации $\Longrightarrow$ теоремы 3.3 , а обратньм импликациям посвяшен следуюший параграфф.

3В. Несчетные $\Pi_{1}^{1}$-множества без совершенного ядра. Здесь доказывается импликация $\Longrightarrow$ в эквивалентности (i) теоремы 3.3. (Обратная импликащия будет доказана ниже в п. 4Б.) Мы доказьваем ее в “контрапозиционной” форме, т.е. для любого $a \in \mathbb{N}^{\omega}$

$$
\mathbf{L}[a] \cap \mathbb{N}^{\omega}-\text { несчетное множество } \Longrightarrow \neg \operatorname{PK}\left(\Pi_{1}^{1}(a)\right) .
$$

ДокАЗАТЕЛьство. Итак, пусть $a \in \mathbb{N}^{\omega}$ и множество $\mathbf{L}[a] \cap \mathbb{N}^{\omega}$ несчетно. Множество $\Xi=\left\{\langle x, w\rangle: w \in \mathbf{W O} \wedge x=\boldsymbol{f}_{|w|}[a]\right\}$ принадлежит $\Pi_{1}^{1}(a)$ : это следует из предложения 1.11(i) и теоремы 2.6(ii). По теореме униформизации 1.9 найдется униформное $\Pi_{1}^{1}(a)$-множество $C \subseteq \Xi$ такое, что $\operatorname{dom} C=\operatorname{dom} \Xi$, другими словами, $C$ - график функции $\eta$ с областью определения $\operatorname{dom} \eta=\mathbf{L}[a] \cap \mathbb{N} \omega$, такой, что $x=\boldsymbol{f}_{|\eta(x)|}[a]$ выполнено для каждого $x \in \mathbf{L}[a] \cap \mathbb{N}^{\omega}$. Таким образом, $\Sigma_{2}^{1}(a)$-множество $R=\operatorname{ran} \eta=\left\{\eta(x): x \in \mathbf{L}[a] \cap \mathbb{N}^{\omega}\right\} \subseteq \mathbf{W O}$ несчетно вместе с $\mathbf{L}[a] \cap \mathbb{N}^{\omega} .^{21}$

Очевидно, что множество $R$ содержит не более одной точки в каждой конституанте $\mathbf{W O}_{\xi}=E_{\xi}(\mathscr{L})$ решета Лебега $\mathscr{L}$ (см. пример 1.10 .1$)$, так что $R$ не содержит совершенных подмножеств. (Если $X \subseteq R$ - совершенное множество, то, по теореме 1.8 , найдется ординал $\vartheta<\omega_{1}$ такой, что $X \subseteq \bigcup_{\xi<\vartheta} E_{\xi}(\mathscr{L})$, противоречие.) Итак, $R$ есть несчетное $\Sigma_{2}^{1}(a)$-множество без совершенного ядра.

Чтобы извлечь отсюда $\Pi_{1}^{1}(a)$-множество с тем же свойством, заметим, что, по теореме 1.9 , найдется униформное $\Pi_{1}^{1}(a)$-множество $C \subseteq \mathbb{N}^{\omega} \times \mathbb{N}^{\omega}$ такое, что $R=$ $\operatorname{dom} C=\{x: \exists y(\langle x, y\rangle \in C)\}$. (Униформность означает, что для каждого $x^{\prime}$ имеется не более одного $y$ с $\left\langle x^{\prime}, y\right\rangle \in C$.) Если $P \subseteq C$ - совершенное множество, то его проекция $A=\operatorname{dom} P \subseteq R$ несчетна, ибо $C$ и $P$ униформны, и является $\boldsymbol{\Sigma}_{1}^{1}$-множеством. По теореме 1.4, найдется совершенное $Y \subseteq A \subseteq R$. Но $R$ не имеет совершенных подмножеств, противоречие.

\footnotetext{
${ }^{21}$ Мы придем к тому же результату, если определим $R$ как множество, состоящее из $\prec a$-наименьших элементов $w_{\xi}$ в каждом $\mathbf{W} \mathbf{O}_{\xi}$. Заметим, что доказательство леммы не обращается прямо к гёделеву полному упорядочению $\prec a$ множества $\mathbf{L}[a] \cap \mathbb{N}^{\omega}$ (хотя, конечно, использует последовательность $\left.\left\{\boldsymbol{f}_{\xi}[a]\right\}_{\xi<\omega_{1}}\right)$.
} 
В [37] дано доказательство сушествовани $\Pi_{1}^{1}$-множества без свойства совершенного ядра (в предположении несчетности $\mathbf{L}[a] \cap \mathbb{N} \omega$ для некоторого $a \in \mathbb{N} \omega$ ), более элементарное в том смысле, что оно не использует решета и конституанты.

3Г. Неизмеримые множества второго проективного уровня. В этом пункте доказываются импликашии $\Longrightarrow$ в (ii)-(v) теоремы 3.3. Мы доказьваем их в "контрапозищионной” форме, т.е.

(ii') Rand L $[a]$ - не множество полной $\boldsymbol{\lambda}$-меры $\Longrightarrow \neg \operatorname{LM}\left(\Sigma_{2}^{1}(a)\right)$;

(iii') $\mathbf{C o h} \mathrm{L}[a]-$ не котошее множество $\Longrightarrow \neg \mathrm{BP}\left(\Sigma_{2}^{1}(a)\right)$;

$\left(\mathrm{iv}^{\prime}\right)$ Rand $\mathbf{L}[a]=\varnothing \Longrightarrow \neg \operatorname{LM}\left(\Delta_{2}^{1}(a)\right)$;

$\left(\mathrm{v}^{\prime}\right) \operatorname{Coh} \mathbf{L}[a]=\varnothing \Longrightarrow \neg \operatorname{BP}\left(\Delta_{2}^{1}(a)\right)$.

ДоказАтельство. (iii') Предположим, что $a \in \mathbb{N}^{\omega}$ и $\mathbf{C o h} \mathbf{L}[a]-$ не котошее множество. Множество $C=\operatorname{cod} \mathscr{I}_{\text {cat }}=\left\{c \in \mathbf{B C}: \boldsymbol{B}_{c} \in \mathscr{I}_{\text {cat }}\right\}$ принадлежит $\Pi_{1}^{1}$ согласно 1.11(vi). Однако, с целью дать такое изложение доказательства, которое будет ниже "работать" и в более общем случае, мы будем исходить в последуюших выкладках только из того, что $C \in \Sigma_{2}^{1}$. Тогда, по теореме 1.9 , найдется $\Pi_{1}^{1}$-множество $P \subseteq \mathbb{N}^{\omega} \times \mathbb{N}^{\omega}$ такое, что $c \in C \Longleftrightarrow \exists y P(c, y)$.

Напомним, что $\boldsymbol{f}_{\xi}[a]$ есть $\xi$-й элемент $\mathbf{L}[a] \cap \mathbb{N}^{\omega}$ в смысле полного упорядочения $\prec_{a}$ множества $\mathbf{L}[a] \cap \mathbb{N}^{\omega}$. Для $\xi<\omega_{1}$ если $\boldsymbol{f}_{\xi}[a]=h$ таково, что $\left\langle(h)_{0},(h)_{1}\right\rangle \in P$ (тогда $\left.(h)_{0} \in C\right),-$ то определим $S_{\xi}=\boldsymbol{B}_{(h)_{0}}$ (борелевское множество с кодом $\left.(h)_{0}\right)$, а иначе пусть $S_{\xi}=\varnothing$. В обоих случаях $S_{\xi} \in \mathscr{I}_{\text {саt. }}$. Положим $X_{\xi}=S_{\xi} \backslash \bigcup_{\eta<\xi} S_{\eta}$. Мы утверждаем, что множества

$$
S=\left\{\langle w, x\rangle: w \in \operatorname{Ord} \wedge x \in S_{|w|}\right\} \quad \text { и } W=\left\{\langle w, x\rangle: w \in \operatorname{Ord} \wedge x \in X_{|w|}\right\}
$$

принадлежат $\Sigma_{2}^{1}(a)$. Действительно, для $S$ это следует из того, что

$$
\langle w, x\rangle \in S \Longleftrightarrow w \in \mathbf{W O} \wedge \exists h\left(h=\boldsymbol{f}_{|w|}[a] \wedge\left\langle(h)_{0},(h)_{1}\right\rangle \in P \wedge x \in \boldsymbol{B}_{(h)_{0}}\right),
$$

где $\mathbf{W O} \in \Pi_{1}^{1}$ и множество $\left\{\left\langle w, \boldsymbol{f}_{|w|}[a]\right\rangle: w \in \mathbf{W O} \wedge a \in \mathbb{N}^{\omega}\right\}$ принадлежит $\Pi_{1}^{1}$ по теореме 2.6(ii). Что же касается $W$, то, в обозначениях примера 1.10.1, соотношение $x \in X_{|w|}$ равносильно тому, что $x \in S_{|w|} \wedge \forall k\left(w(k)=0 \Longrightarrow x \notin S_{|w|_{k} \mid}\right)$. Но тогда

$$
Z=\bigcup_{\eta \leqslant \xi<\omega_{1}}\left(X_{\eta} \times X_{\xi}\right)=\bigcup_{\eta \leqslant \xi<\omega_{1}}\left\{\langle x, y\rangle: x \in X_{\eta} \wedge y \in X_{\xi}\right\}
$$

также является $\Sigma_{2}^{1}(a)$-множеством, так как

$$
\langle x, y\rangle \in Z \Longleftrightarrow \exists w, w^{\prime} \in \mathbf{W O}\left(|w| \leqslant\left|w^{\prime}\right| \wedge\langle w, x\rangle \in W \wedge\left\langle w^{\prime}, y\right\rangle \in W\right),
$$

где $\mathbf{W O}$ и отношение $|w|<\left|w^{\prime}\right|$ принадлежат $\Pi_{1}^{1}$, см. предложение 1.11 .

Мы утверждаем, что множество $Z$ не имеет свойства Бәра в $\mathbb{N}^{\omega} \times \mathbb{N}^{\omega}$. B самом деле, в противном случае $Z$ - тошее по теореме Улама - Куратовского, поскольку каждое сечение $Z^{y}=\{x:\langle x, y\rangle \in Z\}$ есть объединение счетного числа множеств вида $X_{\xi}$, следовательно, тощее множество. С другой стороны, проекция $X=\{x$ : $\exists y(\langle x, y\rangle \in Z)\}=\bigcup_{\xi<\omega_{1}} X_{\xi}$ множества $Z$, очевидно, совпадает с дополнением $D$ множества $\operatorname{Coh} \mathrm{L}[a]$, т.е., в нашей ситуации, $D$ - не тошее множество. А каждое сечение $Z_{x}=\{y:\langle x, y\rangle \in Z\}, x \in X$, отличается от $D$ опять-таки на счетное объединение множеств вида $X_{\xi}$, т.е. тоже не является тошим множеством. Значит, по теореме 
Улама-Куратовского, само $Z$ не может быть тощим множеством, что противоречит сказанному вьше. Итак, $\Sigma_{2}^{1}(a)$-множество $Z \subseteq(\mathbb{N} \omega)^{2}$ не имеет свойства Бэра. Используя рекурсивный гомеоморфизм $\left(\mathbb{N}^{\omega}\right)^{2} \stackrel{\text { на }}{\longrightarrow} \mathbb{N}^{\omega}$, мы переводим этот контрпример в $\mathbb{N}^{\omega}$.

$\left(\mathrm{v}^{\prime}\right)$ Здесь $\mathbf{C o h} \mathbf{L}[a]=\varnothing$, так что множество $Z$ (из предыдущего рассуждения) принадлежит $\Delta_{2}^{1}(a)$ : в самом деле, $Z$ является дополнением множества $Z^{\prime}=$ $\bigcup_{\xi<\eta<\omega_{1}}\left(X_{\eta} \times X_{\xi}\right)$, которое принадлежит $\Sigma_{2}^{1}(a)$ по тем же соображениям, что и $Z$.

$\left(\mathrm{ii}^{\prime}\right)$ и $\left(\mathrm{iv}^{\prime}\right)$. Здесь рассуждения аналогичны предыдущим, только нужно рассматривать идеал $\mathscr{I}_{\boldsymbol{\lambda}}$ вместо $\mathscr{I}_{\text {cat }}$, для переноса контрпримера в $\mathbb{N}^{\omega}$ использовать лемму 1.3 для эффективного класса $\Delta_{2}^{1}(a)$. Воообше, здесь следует рассматривать множества пространства $2^{\omega}$ (поскольку мера $\boldsymbol{\lambda}$ определена на $2^{\omega}$ ), например, определить $S_{\xi}=\boldsymbol{B}_{\boldsymbol{f}_{\xi}[a]} \cap 2^{\omega}$ при $\boldsymbol{f}_{\xi}[a] \in \mathbf{B C}\left(\right.$ см. начало доказательства $\left.\left(\mathrm{v}^{\prime}\right)\right)$. Конечно, теорема Улама-Куратовского заменяется теоремой Фубини. Это, однако, не вносит существенных изменений в выкладки.

$$
\text { (импликации } \Longrightarrow \text { в (ii)-(v) теоремь 3.3) }
$$

3Д. Обобщение: измеримость относительно идеала. Очевидное сходство между утверждениями (ii), (iv) теоремы 3.4, с одной стороны, и (iii), (v), с другой, привело к следуюшему обшему подходу к такого рода эквивалентностям. Пусть $\mathscr{I}-$ идеал в семействе всех борелевских подмножеств $\mathbb{N} \omega$. Назовем множество $X \subseteq \mathbb{N}^{\omega}$ :

$\mathscr{I}$-нулевы.м, если оно накрьвается множеством из $\mathscr{I}$,

$\mathscr{I}$-измеримым, если найдется борелевское множество $U$ такое, что симметрическая разность $X \triangle U$ есть $\mathscr{I}$-нулевое множество, и

$\mathscr{I}$-полныц, если $\mathbb{N} \omega \backslash X$ есть $\mathscr{I}$-нулевое множество.

ОПРЕДЕЛЕНИЕ 3.6. Пусть $\mathfrak{M}$ - транзитивная модель ZFC. Точка $x \in \mathbb{N}^{\omega}$ называется $\mathscr{I}$-случайной над $\mathfrak{M}$, символически $x \in \boldsymbol{R a n d}_{\mathscr{I}} \mathfrak{M}$, если $x \notin \boldsymbol{B}_{c}$ всякий раз, когда $c \in \mathbf{B C} \cap \mathfrak{M}$ и $\boldsymbol{B}_{c} \in \mathscr{I}$.

Назовем $\sigma$-УСА-идеалом любой идеал $\mathscr{I}$ борелевских подмножеств $\mathbb{N} \omega$, удовлетворяюший таким двум условиям:

$1^{\circ}$. Идеал $\mathscr{I}$ замкнут относительно счетных объединений $(\sigma$-аддитивность).

$2^{\circ}$. Каждое семейство попарно $\mathscr{I}$-дизъюнктных (т.е. попарные пересечения принадлежат $\mathscr{I})$ борелевских множеств, еㅡ принадлежаших $\mathscr{I}$, не более чем счетно (б-насыщенность, или условие счетности антицепей, кратко УСА).

Следуюшая теорема обобщает классические результаты об измеримости и свойстве Бэра для классов $\boldsymbol{\Sigma}_{1}^{1}$ и $\Pi_{1}^{1}$ (теорема 1.4$)$.

ТЕОРема 3.7. Если $\mathscr{I}$ есть б-УСА-идеал борелевских подмножеств $\mathbb{N} \omega$, то

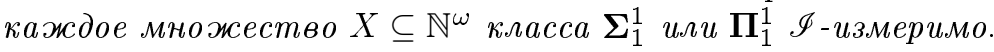

ДокАЗАТЕльство. Для каждого множества $Z \subseteq \mathbb{Q}$ положим $Z^{\prime}=Z \backslash\{q\}$, если $q$ - наименьший элемент $Q$, а если $Z$ не имеет наименьшего элемента, то просто $Z^{\prime}=Z$. Если $R \subseteq \mathbb{N}^{\omega} \times \mathbb{Q}$, то определим $R^{\prime} \subseteq R$ так, что $R^{\prime(x)}=\left(R^{(x)}\right)^{\prime}$ для каждого $x \in \mathbb{N}^{\omega}$. (Напомним, что $R^{(x)}=\{q:\langle x, q\rangle \in R\}$.)

Рассмотрим $\Pi_{1}^{1}$-множество $X=E(R)$, где $R \subseteq \mathbb{N}^{\omega} \times \mathbb{Q}$ - борелевское решето. Индукцией по $\xi<\omega_{1}$ определим $R(\xi) \subseteq R$ так, что $R(\xi+1)=R(\xi)^{\prime}$ и $R(\vartheta)=\bigcap_{\xi<\vartheta} R(\xi)$ для предельных $\vartheta($ а $R(0)=R)$. Легко видеть, что множества $R(\xi)$ и $R_{q}(\xi)=\{x$ : 
$\langle x, q\rangle \in R(\xi)\}(q \in \mathbb{Q})$ - борелевские. Кроме того, мы имеем $R_{q}(\xi) \subseteq R_{q}(\eta)$ при $\eta<\xi$ и любом $q$. Поэтому по выбору $\mathscr{I}$ сушествует ординал $\xi<\omega_{1}$ такой, что $R_{q}(\xi) \backslash R_{q}(\eta) \in \mathscr{I}$ всякий раз, когда $\xi \leqslant \eta$ и $q \in \mathbb{Q}$.

С другой стороны, если $x$ принадлежит множеству $D=X \backslash E_{\leqslant \xi}(R)$, то $\langle x, q\rangle \in$ $R(\xi) \backslash R(\xi+1)$ хотя бы для одного $q$, так что $D \subseteq \bigcup_{q \in \mathbb{Q}} R_{q}(\xi) \backslash R_{q}(\xi+1)$. Значит, множество $D$ является $\mathscr{I}$-нулевым (благодаря $\sigma$-аддитивности идеала $\mathscr{I}$ ). Остается заметить, что $E_{\leqslant \xi}(R)=\bigcup_{\zeta \leqslant \xi} E_{\zeta}(R)$ - борелевское множество.

Следуюшее определение выражает абсолютность свойств $1^{\circ}, 2^{\circ}$ данного идеала $\mathscr{I}$ относительно некоторой транзитивной модели. По понятным причинам абсолютность легче выразить в терминах кодов борелевских множеств, а не самих множеств. В связи с этим, положим $\operatorname{cod} \mathscr{X}=\left\{c \in \mathbf{B C}: \boldsymbol{B}_{c} \in \mathscr{X}\right\}$ для любой совокупности $\mathscr{X}$ борелевских множеств в $\mathbb{N}^{\omega}$. Скажем, что $\mathscr{X}$ является $\mathbf{K}$-в-кодах (где $\mathbf{K}$ - какой-то класс точечных множеств), если $\operatorname{cod} \mathscr{X}$ принадлежит $\mathbf{K}$.

Пусть $\mathfrak{M}$ - транзитивная модель ZFC (например, класс вида $\mathbf{L}[a], a \in \mathbb{N}^{\omega}$ ). Назовем $\sigma$-УСА-идеал $\mathscr{I} \mathfrak{M}$-абсолютныц, если выполнено

$3^{\circ}$ Множество $C^{\prime}=\operatorname{cod} \mathscr{I} \cap \mathfrak{M}$ принадлежит $\mathfrak{M}$ и свойства $1^{\circ}, 2^{\circ}$ абсолютны для $\mathfrak{M}$, т.е. в $\mathfrak{M}$ истинно, что $\mathscr{I}^{\prime}=\left\{\boldsymbol{B}_{c}: c \in C\right\}$ является $\sigma$-УСА-идеалом.

ЛЕмма 3.8. Пусть $\mathfrak{M}$ - любая транзитивная модель ZFC. $K \mathfrak{M}$-абсолютныц б-УСА-идеалам относятся:

- идеал $\mathscr{I}_{\text {сат }}$ всех тощих борелевских множеств $X \subseteq \mathbb{N}^{\omega}$;

- идеал $\mathscr{I}_{\mu}$ всех борелевских $X \subseteq \mathbb{N}^{\omega} \mu$-меры 0, если $\mu$ - борелевска мера на $\mathbb{N}^{\omega}$, код которой, т.е. функиия $\operatorname{cod}(\mu)(n)=\mu\left(\mathscr{N}_{s_{n}}\right)$, принадлежит $\mathfrak{M}$;

- в частности, идеал $\mathscr{I}_{\boldsymbol{\lambda}}$ всех борелевских множеств $X \subseteq \mathbb{N}^{\omega} c \boldsymbol{\lambda}(X)=0$.

Идеаль $\mathscr{I}_{\text {сат }}$ и $\mathscr{I}_{\boldsymbol{\lambda}}$ являются $\Pi_{1}^{1}$-в-кодах, а идеал $\mathscr{I}_{\mu}-\Pi_{1}^{1}(\operatorname{cod}(\mu))$-в-кодах.

ДокАЗАТЕльство. Для идеала $\mathscr{I}_{\text {cat }}$ множество $C=\operatorname{cod} \mathscr{I}_{\text {саt }}$ принадлежит $\Pi_{1}^{1}$ согласно $1.11(\mathrm{vi})$, так что мы имеем свойство $\Pi_{1}^{1}$-в-кодах для $\mathscr{I}_{\text {cat }}$. Поэтому, по теореме абсолютности 2.8 , множество $C^{\prime}=C \cap \mathfrak{M}$ принадлежит $\mathfrak{M}$ и в $\mathfrak{M}$ истинно " $C^{\prime}=\left\{c \in \mathbf{B C}: \boldsymbol{B}_{c}\right.$ - тошее $\}=\mathscr{I}_{\text {cat" }}$. Отсюда мы имеем $3^{\circ}$, поскольку свойства $1^{\circ}, 2^{\circ}$ для $\mathscr{I}_{\text {саt }}$ являются теоремами $\mathbf{Z F C}$, а потому вьполнены в $\mathfrak{M}^{\prime}$.

Идеалы $\mathscr{I}_{\boldsymbol{\lambda}}, \mathscr{I}_{\mu}$ рассматриваются аналогично, только $1.11(\mathrm{v})$ (и его аналог для произвольной меры $\mu$ ) используется вместо 1.11(vi).

Рассматривается следуюшая гипотеза $\mathscr{I}$-измеримости для данного (например, проективного) класса $\mathbf{K}$ и данного $\sigma$-УСА-идеала $\mathscr{I}$ :

$\mathrm{M}_{\mathscr{I}}(\mathbf{K})$ : все множества $X \subseteq \mathbb{N}^{\omega}$ из $\mathbf{K} \mathscr{I}$-измеримы.

Например, мы имеем $\mathrm{M}_{\mathscr{I}}\left(\boldsymbol{\Sigma}_{1}^{1}\right), \forall \mathscr{I}$, по теореме 3.7 .

3Е. Множества, неизмеримые относительно идеала. Ниже будет доказана

ТеОРема 3.9. Пусть $a \in \mathbb{N}^{\omega} \quad u \quad \mathscr{I}$ есть $\mathbf{L}[a]$-абсолютный $\sigma$-УСА-идеал,

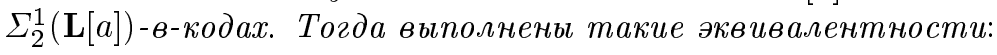

(a) $\mathrm{M}_{\mathscr{I}}\left(\Sigma_{2}^{1}(\mathbf{L}[a])\right) \Longleftrightarrow \operatorname{Rand}_{\mathscr{I}} \mathbf{L}[a]$ является $\mathscr{I}$-полныцм множеством;

(б) $\mathrm{M}_{\mathscr{I}}\left(\Delta_{2}^{1}(\mathbf{L}[a])\right) \Longleftrightarrow \mathbf{R a n d}_{\mathscr{I}} \mathbf{L}[a] \cap \boldsymbol{B}_{c} \neq \varnothing$, каково бь ни бьло борелевское множество $\boldsymbol{B}_{c} \notin \mathscr{I}$ с кодом $c \in \mathbf{L}[a] \cap \mathbf{B C}$. 
ЗАмЕЧАНИЕ 3.10. О взаимоотношениях этой теоремы с теоремой 3.3 и следствием 3.4. Понятно, что правая часть (а) тождественна правым частям (ii), (iii) теоремы 3.3 при, соответственно, $\mathscr{I}=\mathscr{I}_{\boldsymbol{\lambda}}$ и $\mathscr{I}=\mathscr{I}_{\text {cat }}$. (Заметим, что оба идеала находятся в области действия теоремы 3.9 согласно лемме 3.8.) Правая часть (б) вьглядит сильнее правых частей (iv), (v) теоремы 3.3 (для идеалов $\mathscr{I}_{\boldsymbol{\lambda}}, \mathscr{I}_{\text {cat }}$ ), но на самом деле равносильна последним согласно лемме $3.2(1),(2)$. Левые же части (а) и (б) несомненно включают левые части, соответственно, (ii), (iii) и (iv), (v).

Таким образом, поскольку импликации $\Longrightarrow$ в эквивалентностях (ii)-(v) теоремы 3.3 уже доказаны в п. 3Г, теорема 3.9 не дает ничего нового в направлении $\Longrightarrow$ для идеалов $\mathscr{I}_{\boldsymbol{\lambda}}, \mathscr{I}_{\text {cat }}$. Ее область действия, однако, много шире, поскольку $\sigma$-УСА-идеалы, обладающие свойствами, о которых идет речь в теореме, отнюдь не исчерпьваются двумя указанными идеалами. Доказательство импликаций $\Longrightarrow$ теоремы 3.9 дается ниже в этом пункте.

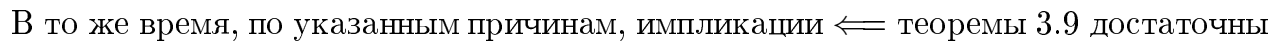
для вьвода импликаций є в эквивалентностях (ii)-(v) теоремы 3.3. Импликации этого направления доказываются в 4 с помошюю метода вынуждения.

Отметим наконец, что теорема 3.9 (для идеалов $\mathscr{I}_{\boldsymbol{\lambda}}, \mathscr{I}_{\text {cat }}$ ) влечет следствие $3.4(\mathrm{II})-$ (V) по очевидныт соображениям.

Для доказательства импликаций $\Longrightarrow$ в теореме 3.9 предполагаем, что $\mathscr{I}$ является $\sigma$-УСА-идеалом, а множество $C=\operatorname{cod} \mathscr{I}$ принадлежит $\Sigma_{2}^{1}(\mathbf{L}[a])$. Искомьй результат удобно представить в контрапозиционной форме:

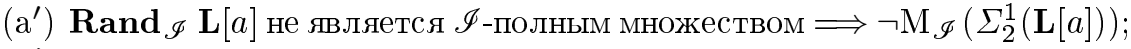

$\left(\sigma^{\prime}\right) \boldsymbol{R a n d}_{\mathscr{I}} \mathbf{L}[a] \cap \boldsymbol{B}_{c}=\varnothing$ для некоторого борелевского множества $\boldsymbol{B}_{c} \notin \mathscr{I}$ с кодом $c \in \mathbf{L}[a] \cap \mathbf{B C} \Longrightarrow \neg \mathbf{M}_{\mathscr{I}}\left(\Delta_{2}^{1}(\mathbf{L}[a])\right) ;$

Прежде всего отметим, что рассуждение в п. ЗГ здесь не проходит в самой последней части, где $\mathscr{I}$-неизмеримое множество в $\mathbb{N}^{\omega}$ извлекается из $\mathscr{I}^{2}$-неизмеримого подмножества $\mathbb{N}^{\omega} \times \mathbb{N} \omega$. (Под $\mathscr{I}^{2}$ имеется в виду произведение Фубини.) Для идеалов $\mathscr{I}_{\boldsymbol{\lambda}}$ и $\mathscr{I}_{\text {саt }}$ возможность такого перехода от $\mathbb{N}^{\omega} \times \mathbb{N}^{\omega}$ к $\mathbb{N}^{\omega}$ достаточно очевидна, но для произвольного $\sigma$-УСА-идеала $\mathscr{I}$ вряд ли возможна. К счастью, имеется другое рассуждение, производяшее контрпримеры непосредственно в $\mathbb{N}^{\omega}$. Результат, правда, не столь точен, как конструкция п. $3 \Gamma$ : даже если $C$ есть $\Sigma_{2}^{1}(a)$-множество, то контрпримеры получаются все равно в классе $\Sigma_{2}^{1}(\mathbf{L}[a])$.

$\left(\mathrm{a}^{\prime}\right)$ Раз $C \in \Sigma_{2}^{1}(\mathbf{L}[a])$, найдется $\Pi_{1}^{1}(\mathbf{L}[a])$-множество $P \subseteq \mathbb{N}^{\omega} \times \mathbb{N}^{\omega}$ такое, что $c \in C \Longleftrightarrow \exists y P(c, y)$. Исходя из этого множества $C$, определим $S_{\xi} \in \mathscr{I}, X_{\xi}, S, W$ как в п. $3 \Gamma$ - теперь, конечно, $S$ и $W$ суть $\Sigma_{2}^{1}(\mathbf{L}[a])$-множества по выбору $C$.

Далее, мы имеем $\omega_{1}^{\mathbf{L}[a]}=\omega_{1}$. (В противном случае множество $\mathbf{L}[a] \cap \mathbb{N}^{\omega}$ счетно, a

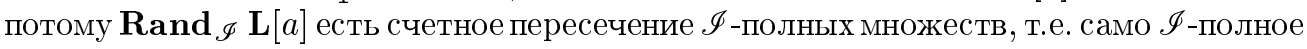
множество, что противоречит нашим предположениям.) Значит, для любого $\xi<\omega_{1}=$ $\omega_{1}^{\mathbf{L}[a]}$ найдется $b \in \mathbf{L}[a] \cap \mathbb{N}^{\omega}$ такое, что

$$
\left\{(b)_{k}: k \in \mathbb{N}\right\} \subseteq \mathbf{W O} \text { и }\left\{\left|(b)_{k}\right|: k \in \mathbb{N}\right\}=\xi \cup\{\xi\}
$$

причем если $\xi \geqslant \omega$, то $\left|(b)_{k}\right| \neq\left|(b)_{k^{\prime}}\right|$ при $k \neq k^{\prime}$. (Такое $b$ кодирует пересчет всех ординалов $\leqslant \xi$, причем без повторений при $\xi \geqslant \omega$.) Через $b_{\xi}$ обозначим $\prec{ }_{a}$-наименьшее 
из таких $b$. Наконец, определим

$$
\Xi_{\eta k}=\left\{\xi<\omega_{1}:\left|\left(b_{\xi}\right)_{k}\right|=\eta\right\}, \quad Y_{\eta k}=\bigcup_{\xi \in \Xi_{\eta k}} X_{\xi}, \quad Y_{\eta}=\bigcup_{k} Y_{\eta k}
$$

Понятно, что $Y_{\eta}=\bigcup_{\xi \geqslant \eta} X_{\xi}$, в частности, $Y_{0}=\bigcup_{\xi<\omega_{1}} X_{\xi}$ есть не что иное, как дополнение $\mathbb{N}^{\omega} \backslash \boldsymbol{R a n d}_{\mathscr{I}} \mathbf{L}[a]$ множества $\operatorname{Rand}_{\mathscr{I}} \mathbf{L}[a]$. Значит, в наших допушениях, $Y_{0}$ не есть $\mathscr{I}$-нулевое множество. Предположим (с целью вьвести противоречие), что все множсества $Y_{\eta k}$ (тогда и все $Y_{\eta}$ ) $\mathscr{I}$-измеримы. Ни одно из $Y_{\eta}$ не может быть $\mathscr{I}$-нулевым множеством, поскольку $Y_{0} \backslash Y_{\eta}=\bigcup_{\zeta \leqslant \eta} X_{\zeta}$ есть счетное объединение множеств $\mathscr{I}$-нулевых множеств (используется свойство $1^{\circ}$ идеала $\mathscr{I}$ ). Поэтому (снова $1^{\circ}$ !) для каждого $\eta<\omega_{1}$ найдется число $k_{\eta}$ такое, что $Y_{\eta k_{\eta}}$ - не $\mathscr{I}$-нулевое множество, а тогда найдется $k$ такое, что множество $H_{k}=\left\{\eta: k_{\eta}=k\right\}$ несчетно. Тогда $Y_{\eta k}$ - не $\mathscr{I}$-нулевое множество для всех $\eta \in H_{k}$. Для получения противоречия со свойством $2^{\circ}$ идеала $\mathscr{I}$ остается заметить, что $Y_{\eta k} \cap Y_{\eta^{\prime} k}=\varnothing$ при $\eta^{\prime} \neq \eta$. (Если $x \in Y_{\eta k} \cap Y_{\eta^{\prime} k}$, то найдутся ординалы $\xi \neq \xi^{\prime}$ такие, что $\eta=\left(b_{\xi}\right)_{k}, \eta^{\prime}=\left(b_{\xi^{\prime}}\right)_{k}$, $x \in X_{\xi} \cap X_{\xi^{\prime}}$, что невозможно, так как множества $X_{\xi}$ попарно дизъюнктны.)

Итак, не все множества $Y_{\eta k}$ могут быть $\mathscr{I}$-измеримыми. Остается проверить, что каждое $Y_{\eta k}$ принадлежит $\Sigma_{2}^{1}(\mathbf{L}[a])$. Множества

$$
B=\left\{\left\langle w, b_{|w|}\right\rangle: w \in \operatorname{Ord}\right\} \quad \text { и } \Omega=\left\{\langle t, w, k\rangle: t, w \in \mathbf{W O} \wedge|t| \in \Xi_{|w| k}\right\}
$$

принадлежат $\Sigma_{2}^{1}(a)$ по теореме 2.6(iv). Возьмем произвольные $\eta<\omega_{1}$ и $k$ и выберем $w \in \mathbf{W O}_{\eta} \cap \mathbf{L}[a]$. Тогда $T=\{t \in \mathbf{W O}:\langle t, w, k\rangle \in \Omega\}$ есть $\Sigma_{2}^{1}(a, w)$-множество. Поэтому $Y_{\eta k}=\{x: \exists t \in T(\langle t, x\rangle \in W\})$ принадлежит $\Sigma_{2}^{1}(\mathbf{L}[a])$ вместе с $W$.

$\left(\sigma^{\prime}\right)$ Здесь мы предполагаем, что $c \in \mathbf{B C} \cap \mathbf{L}[a], \boldsymbol{B}_{c} \notin \mathscr{I}_{\text {и }} \mathbf{R a n d}_{\mathscr{I}} \mathbf{L}[a] \cap \boldsymbol{B}_{c}=\varnothing$. Повторим конструкцию из доказательства (a), начав ее, однако, с определени $S_{\xi}=$ $\boldsymbol{B}_{(h)_{0}} \cap \boldsymbol{B}_{c}$ в случае, когда $\left\langle(h)_{0},(h)_{1}\right\rangle \in P$, а иначе $S_{\xi}=\varnothing$. В результате получим: $Y_{\eta k} \subseteq \boldsymbol{B}_{c}$, и не все множества $Y_{\eta k}$ являются $\mathscr{I}$-измеримыми. При этом каждое $Y_{\eta k}$ принадлежит $\Sigma_{2}^{1}(\mathbf{L}[a])$. Остается показать, что каждое $Y_{\eta k}$ является также множеством класса $\Pi_{2}^{1}(\mathbf{L}[a])$. В рассматриваемом случае $Y_{0}=\boldsymbol{B}_{c}$, а потому каждое $Y_{\eta}=Y_{0} \backslash \bigcup_{\xi<\eta} X_{\xi}$ есть борелевское множество. Кроме того, при $\eta \geqslant \omega$ мы имеем $Y_{\eta k} \cap Y_{\eta k^{\prime}}=\varnothing$ при $k \neq k^{\prime}$, поскольку, по построению, $\left|\left(b_{\xi}\right)_{k}\right| \neq\left|\left(b_{\xi}\right)_{k^{\prime}}\right|$ при $k \neq k^{\prime}$, а множества $X_{\xi}$ попарно дизъюнктны. Значит, $Y_{\eta k}=Y_{\eta} \backslash \bigcup_{k^{\prime} \neq k} Y_{\eta k^{\prime}}$ есть $\Pi_{2}^{1}$-множество. Более точньй анализ, основанный на результатах для множеств $\Omega$ и $W$, позволяет найти, что $Y_{\eta k} \in \Pi_{2}^{1}(\mathbf{L}[a])$, тем же способом, что и в последней части доказательства (a). А значениями $\eta<\omega$ здесь можно пренебречь.

$\square$ (импликации $\Longrightarrow$ в теореме 3.9$)$

\section{3Ж. Непротиворечивость существования таких множеств.}

Теорема 3.11. Если $\mathbb{N}^{\omega} \subseteq \mathbf{L}[a]$ для некоторого а $\in \in \mathbb{N}^{\omega}$, то не выполняются $\operatorname{PK}\left(\Pi_{1}^{1}(a)\right), \operatorname{BP}\left(\Delta_{2}^{1}(a)\right), \operatorname{LM}\left(\Delta_{2}^{1}(a)\right)$. Следовательно, конбюнкиия отричаний гипотез $\mathrm{PK}\left(\Pi_{1}^{1}\right), \operatorname{BP}\left(\Delta_{2}^{1}\right), \operatorname{LM}\left(\Delta_{2}^{1}\right)$ (другими словами, утверждение о существовании контрпримеров в соответствующих классах) не противоречит аксиомам ZFC. 
ДокАЗАТЕльство. В наших предположениях множество $\mathbf{L}[a] \cap \mathbb{N}^{\omega}=\mathbb{N}^{\omega}$ несчетно, более того, каждое одноэлементное множество $\{x\}, x \in \mathbb{N}^{\omega},-$ борелевское множество с кодом из $\mathbf{L}[a]$, тощее и меры 0 , так что мы имеем $\operatorname{Coh} \mathbf{L}[a]=\operatorname{Rand} \mathbf{L}[a]=\varnothing$. Teперь нарушение $\operatorname{LM}\left(\Delta_{2}^{1}(a)\right)$ и $\mathrm{BP}\left(\Delta_{2}^{1}(a)\right)$ следует из доказанного в п. $3 \Gamma$, а нарушение $\operatorname{PK}\left(\Pi_{1}^{1}(a)\right)$ - из доказанного в п. 3В.

Для доказательства второй части теоремы отметим, что аксиома конструктивности $\mathbf{V}=\mathbf{L}$ влечет $\mathbb{N}^{\omega} \subseteq \mathbf{L}[a]$ для любого $a$, а с другой стороны, аксиома конструктивности не противоречит $\mathbf{Z F C}$ по Гёделю.

Доказанная теорема может быть уточнена в отношении свойства РК. Рассмотрим следуюший вариант гипотезы $\mathrm{PK}(\mathbf{K})$ :

$\mathrm{PK}^{-}(\mathbf{K})$ : всякое $\mathbf{K}$-множество $P \subseteq \mathbb{N}^{\omega} \times \mathbb{N}^{\omega}$, являющееся графиком всюду определенной функции из $\mathbb{N}^{\omega}$ в $\mathbb{N} \omega$ (тогда по необходимости несчетное), содержит совершенное подмножество.

ЛЕМма 3.12. Если $a \in \mathbb{N}^{\omega} u \mathbb{N}^{\omega} \subseteq \mathbf{L}[a]$, то не выполняется $\mathrm{PK}^{-}\left(\Pi_{1}^{1}(a)\right)$.

ДокАЗАТЕЛЬСтво. Возврашаясь к доказательству в п. 3В, заметим, что в предположении $\mathbb{N}^{\omega} \subseteq \mathbf{L}[a]$ построенная там функция $\eta$ удовлетворяет $\mathbb{N}^{\omega}=\operatorname{dom} \eta$, так что мы имеем $\neg \mathrm{PK}^{-}\left(\Pi_{1}^{1}(a)\right)$.

Исторические и библиографические замечания. Понятие коэновских и случайных точек, как в определении 3.1, принадлежит Соловею [89]; впрочем, коэновские точки с другим, но эквивалентным определением введены Коэном [15] - это первьй и наиболее простой пример объектов, даваемых форсингом. О связи коэновских и случайных точек с форсингом см. п. 4В.

Доказательство теоремы 3.7 использует метод Селивановского [83]; более ранние отдельные доказательства измеримости по Лебегу и свойства Бэра для $\boldsymbol{\Sigma}_{1}^{1}$-множеств (например, в [57]) отличаются друг от друга и не обобшаются до результата 3.7.

О теореме 3.3 и следствии 3.4. (Напомним, что доказательства импликаций них, а также в теореме 3.9, отложены до следуюшего параграфа.) Эквивалентность (I) приведена в обзоре [74] со ссылкой на Мэнсфилда и Соловея; их доказательства опубликованы позже в [72], [88]. Результат был независимо получен Любецким [17], [65], [67]. Еще раньше Любецкий [64] доказал импликацию $\Longrightarrow$.

Эквивалентностей (II), (III) в [74] нет. Насколько нам известно, (II) впервые была приведена Любецким в [65] (полное доказательство (II) и (III) содержится в [68]). Обе эти эквивалентности нередко связьваются с неопубликованными работами Соловея 60-х годов (иногда, как например в [9; с. 457], дается ссылка на статью [88], где, однако, этих результатов просто нет, а измеримость и свойство Бэра вообще не рассматриваются).

Эквивалентности (IV), (V) следствия 3.4 доказаны Любецким [68], [69] (и включены в обзор [35]). Эти работы, однако, никогда не переводились с русского, а потому результаты остались неизвестными для западных специалистов по теории множеств. Джюдах и Шелах передоказали их в [28].

Теперь о более ранних результатах о существовании контрпримеров. Гёдель объявил в [20], что из аксиомы конструктивности $\mathbf{V}=\mathbf{L}$ (т.е. предположения о том, что все множества принадлежат $\mathbf{L}$ ) вытекает существование неизмеримого $\boldsymbol{\Delta}_{2}^{1}$-множества и несчетного $\Pi_{1}^{1}$-множества без совершенного ядра. Доказательство этой теоремы (т.е. теоремы 3.11 для $\mathbf{L}$ вместо $\mathbf{L}[a]$ ) впервые появилось в статье П. С. Новикова [78]. 
Новиковский контрпример для совершенного ядра воспроизведен в п. 3В и лемме 3.12 с несушественными изменениями. (Следуя [78], мы должны были бы определить, в п. 3В, $\Xi$ как множество всех пар $\{x, w\}$ таких что $w \in \mathbf{W O}$ и $x \in\left\{\boldsymbol{f}_{\xi}[a]\right.$ : $\xi<|w|\}$. Кроме того, Новиков рассматривает "абсолютную” конструктивность, т.е. $\mathbf{L}$ вместо $\mathbf{L}[a]$. Но это не меняет существа рассуждений.) Данньй в [78] контрпример для $\operatorname{LM}\left(\boldsymbol{\Delta}_{2}^{1}\right)$ (опять в предположении $\mathbf{V}=\mathbf{L}$ ) можно представить в следующем виде: если функция $\eta: \mathbb{N}^{\omega} \rightarrow \mathbb{N}^{\omega}$ индуцирует контрпример к $\mathrm{PK}^{-}\left(\boldsymbol{\Pi}_{1}^{1}\right)$, то множество $\left\{\langle x, y\rangle: y<_{\text {lex }} \eta(x)\right\}$, где $<_{\text {lex }}-$ лексикографический порядок на $\mathbb{N} \omega$, является неизмеримым $\boldsymbol{\Delta}_{2}^{1}$-множеством (при условии, что мера обладает тем свойством, что любой бэровский интервал имеет ненулевую меру).

Конструкция контрпримеров для меры и категории в классах $\boldsymbol{\Delta}_{2}^{1}, \boldsymbol{\Sigma}_{2}^{1}$, как в п. $3 \Gamma$, известна из многих источников (например, [10; с. 453] или [92]) и обычно дается со ссылкой на неопубликованные работы Соловея 60-х годов (в [88], [89] этой конструкции и этих результатов нет). Идея обобщенного подхода из п. 3Д и, в частности, метод, использованный в п. 3Е, взяты из [69]. Мы увидим ниже в п. 9В, что имеются другие идеалы, отличные от идеала тощих множеств и идеалов нулевой меры, а также их пересечений, к которьм может быть применена теорема 3.9.

\section{§. Резольвенты классических проблем: вторая часть}

В $\S 3$ мы доказали импликации $\Longrightarrow$ в теоремах 3.3 и 3.9 и в следствии 3.4. Этим устанавливается (теорема 3.11 , со ссылкой на гёделевский результат о непротиворечивости аксиомы конструктивности $\mathbf{V}=\mathbf{L}$ ), что отрицательные решения гипотез, рассматриваемых в следствии 3.4 (т.е. утверждения о сушествовании контрпримеров к левьг частям всех пяти эквивалентностей), не противоречат аксиомам ZFC. Этот

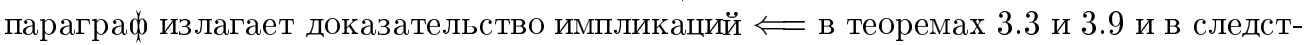
вии 3.4 .

Доказательство использует форсинг (метод вьнуждения). Имеюшийся опыт элиминации форсинга из доказательств утверждений вроде теорем 3.3 и 3.9, т.е. 르 peзультатов о независимости, вряд ли позволяет ожидать получения здесь простых и свободных от форсинга доказательств. Однако, если говорить только об импликациях из диаграммы во введении, исходяших из блока $\mathrm{PK}\left(\boldsymbol{\Pi}_{1}^{1}\right)$, то роль форсинга может быть сведена к единственному утверждению: $\omega_{1}^{\mathbf{L}[a, x]}=\omega_{1}^{\mathbf{L}[a]}$ всякий раз, когда $x \in \mathbf{R a n d} \mathrm{L}[a]$ или $x \in \mathbf{C o h} \mathbf{L}[a]$ (лемма 4.12), а само доказательство этих импликаций завершается в п. 4 Д.

Что же касается непротиворечивости положительных решений гипотез, то она будет установлена в $\S 7$, причем в более сильной форме (свойства регулярности для всех ROD-множеств и, в частности, для всех проективных множеств).

4A. Основы форсинга. Предполагается некоторое знакомство читателя с форсингом, так что нижеследующее можно назвать скорее напоминанием, чем самодостаточным изложением основ этого метода.

На протяжение п. 4 А фиксируем транзитивное множество или класс $\mathfrak{M}$, в котором вьполнены все аксиомы ZFC, - такое $\mathfrak{M}$ называется исходной моделью. Например, $\mathfrak{M}$ может быть счетной моделью, классом вида $\mathbf{L}[x]$ или даже универсумом всех множеств V . Допустим, что $\mathbb{P} \in \mathfrak{M}$ - частично упорядоченное (ЧУ) множество, собственно и называемое форсингом. Элементы $\mathbb{P}$ назьваются (вынужсдающими) условиями. 


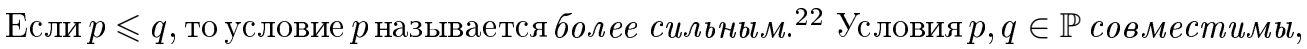
если найдется $r \in \mathbb{P}$ такое, что $r \leqslant p$ и $r \leqslant q$; иначе $p, q$ несовместимы. Множество $A \subseteq \mathbb{P}$ назьвается:

плотнылм (в $\mathbb{P})$, когда $\forall p \in A \exists q \in A(q \leqslant p) ;$

антицепью $($ в $\mathbb{P})$, когда любые $p \neq q \in A$ несовместимы.

Наконец, множество $G \subseteq \mathbb{P}$ назьвается $\mathbb{P}$-генерическим над $\mathfrak{M}$, если

1) $G \cap D \neq \varnothing$ для любого плотного множества $D \subseteq \mathbb{P}, D \in \mathfrak{M}$;

2) если $p \in G, q \in \mathbb{P}, p \leqslant q$, то $q \in G$.

Определим, при помоши $\in$-индукщии по $t, t[G]=\{s[G]: \exists p \in G(\langle p, s\rangle \in t)\}$. Множества $t$, встречаюшиеся в тексте в составе выражений вида $t[G]$, принято назьвать именами. Например, положим $\breve{x}=\{\langle p, \breve{y}\rangle: p \in \mathbb{P} \wedge y \in x\}$ (опять по $\in$-индукции) понятно, что $\breve{x}[G]=x$ для любого множества $x$. Такое $\breve{x}$ назьвается каноническим $\mathbb{P}$-именем $x$. Обычным соглашением является отождествление $\breve{x}$ и $x$ в тех случаях, когда это не приводит к двусмысленности. Мы будем иногда проводить такое отождествление для случая, когда $x$ принадлежит $\mathbb{N}, \mathbb{N}<\omega$ и Ord.

ТеОрема 4.1. Если $\mathbb{P} \in \mathfrak{M}-$ ЧУ множество и множество $G \subseteq \mathbb{P}$ является $\mathbb{P}$-генерическим над $\mathfrak{M}$, то существует единственное транзитивное множество или класс $\mathfrak{M}[G]$ (назьваемое $\mathbb{P}$-генерическим расширением модели $\mathfrak{M}$ ) такое, чmo

(i) $\mathfrak{M} \subseteq \mathfrak{M}[G], G \in \mathfrak{M}[G]$, ординальи $\mathfrak{M}$ и $\mathfrak{M}[G]$ совпадают и все аксиомы ZFC вьлолнень в $\mathfrak{M}[G]$;

(ii) $\mathfrak{M}[G]$ - минимальньй класс, удовлетворяющий (i). Если $x \in \mathfrak{M}[G]$, то найдется имя $t \in \mathfrak{M}$ такое, что $x=t[G]$.

Теорема 4.2 сообшает несколько наиболее важных свойств генерических расширений, т.е. классов вида $\mathfrak{M}[G]$, где множество $G$ является $\mathbb{P}$-генерическим над $\mathfrak{M}$ для какого-нибудь форсинга $\mathbb{P} \in \mathfrak{M}$. Сначала приведем необходимые определения.

1) ЧУ множество $\mathbb{P}$ удовлетворяет $\varkappa$-условию антицепей ( $\varkappa$ - бесконечньй кардинал), если каждая антицепь $A \subseteq \mathbb{P}$ имеет мошность $<\varkappa$ (строго), а условие счетности антицепей, кратко УСА, - это ऍ-условие антищепей.

2) Скажем, что ЧУ множества $\mathbb{P}, \mathbb{P}^{\prime}$ слабо изоморфны, если найдутся порядково изоморфные друг другу плотные множества $D \subseteq \mathbb{P}, D^{\prime} \subseteq \mathbb{P}^{\prime}$.

Tеорема 4.2. Пусть $\mathbb{P}, \mathbb{P}^{\prime} \in \mathfrak{M}-$ два ЧУ множества. Тогда

(i) если множество $G \subseteq \mathbb{P}$ является $\mathbb{P}$-генерическим над $\mathfrak{M}, \varkappa-$ регулярныи й

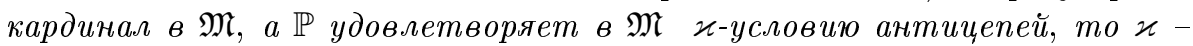
регулярный кардинал и в $\mathfrak{M}[G]$ и, в частности, если $\mathbb{P}$ удовлетворяет УСА в $\mathfrak{M}$, то все кардиналь $\mathfrak{M}$ остаются кардиналами в $\mathfrak{M}[G] ;$

(ii) если $\mathbb{P}, \mathbb{P}^{\prime}$ слабо изоморфнь в $\mathfrak{M}$, то любое $\mathbb{P}$-генерическое расширение $\mathfrak{M}$ является и $\mathbb{P}^{\prime}$-генерическим расширением $\mathfrak{M} ;$

(iii) каждое $\mathbb{P} \times \mathbb{P}^{\prime}$-генерическое над $\mathfrak{M}$ множество имеет вид $G \times G^{\prime}$, где множество $G \subseteq \mathbb{P}$ является $\mathbb{P}$-генерическим над $\mathfrak{M}$, а $G^{\prime} \subseteq \mathbb{P}^{\prime}$ является $\mathbb{P}^{\prime}$-генерическим над $\mathfrak{M}[G]$ ( и над $\left.\mathfrak{M}\right) ;$ при әтом $\mathfrak{M}[G] \cap \mathfrak{M}\left[G^{\prime}\right]=\mathfrak{M}$;

${ }^{22}$ Т.е. вынуждающим больше свойств генерических расширений (см. ниже о последних). Это стандартное (хотя отнюдь не общепринятое - см., например, [10]) соглашение не всегда согласуется с интуицией, посколшку для многих форсингов отношение порядка $\leqslant$ оказьвается обратньм к отношению включения $\subseteq$. Но к этому нужно привыкнуть. 
(iv) обратно, если множество $G \subseteq \mathbb{P}$ является $\mathbb{P}$-генерическим над $\mathfrak{M}$, а мно жество $G^{\prime} \subseteq \mathbb{P}^{\prime}$ является $\mathbb{P}^{\prime}$-генерическим над $\mathfrak{M}[G]$, то множество $G^{\prime} \times G^{\prime \prime}$ является $\mathbb{P}^{\prime} \times \mathbb{P}^{\prime \prime}$-генерическим над $\mathfrak{M .}$

Утверждения (iii), (iv) теоремы 4.2 известны как теорема о произведении.

В теории форсинга определяется отношение вьнуждения, или форсинга, типично обозначаемое $p \Vdash_{\mathbb{P}}^{\mathfrak{M}} \varphi\left(t_{1}, \ldots, t_{n}\right)$ (словами: $p$ вьнуждает $\left.\varphi\left(t_{1}, \ldots, t_{n}\right)\right)$, где $\mathbb{P} \in \mathfrak{M}$ есть ЧУ множество, $p \in \mathbb{P}, t_{1}, \ldots, t_{n} \in \mathfrak{M}$ понимаются как имена, а $\varphi\left(x_{1}, \ldots, x_{n}\right)-$ любая $\in$-формула, т.е. формула языка ZFC. Это отношение удовлетворяет следующей теореме.

ТЕОРема 4.3. Предположим, что, в указанных условиях $\varphi\left(x_{1}, \ldots, x_{n}\right)$ - произвольная $\in$-формула. Тогда

(i) вынуждение формуль $\varphi$ определимо в $\mathfrak{M}$, в том смысле, что следуюшая совокупность определима в $\mathfrak{M} \in$-формулой с параметром $\mathbb{P}$ :

$$
\left\{\left\langle p, t_{1}, \ldots, t_{n}\right\rangle: p \in \mathfrak{M} \wedge t_{1}, \ldots, t_{n} \in \mathfrak{M} \wedge p \Vdash_{\mathbb{P}}^{\mathfrak{M}} \varphi\left(t_{1}, \ldots, t_{n}\right)\right\}
$$

(ii) если $G \subseteq \mathbb{P}-\mathbb{P}$-генерическое над $\mathfrak{M}$, а $t_{1}, \ldots, t_{n} \in \mathfrak{M}-$ имена, то

$$
\varphi\left(t_{1}[G], \ldots, t_{n}[G]\right) \text { истинно в } \mathfrak{M}[G] \Longleftrightarrow \exists p \in G\left(p \Vdash_{\mathbb{P}}^{\mathfrak{M}} \varphi\left(t_{1}, \ldots, t_{n}\right)\right)
$$

(iii) мнохество $\left\{p \in \mathbb{P}: p \Vdash_{\mathbb{P}}^{\mathfrak{M}} \varphi\left(t_{1}, \ldots, t_{n}\right) \vee p \Vdash_{\mathbb{P}}^{\mathfrak{M}} \neg \varphi\left(t_{1}, \ldots, t_{n}\right)\right\}$ плотно в $\mathbb{P}$ и принадлежит $\mathfrak{M}$ каковы бь ни были имена $t_{j} \in \mathfrak{M}$.

Запись $\Vdash_{\mathbb{P}}^{\mathfrak{M}} \varphi$ означает, что $p \Vdash_{\mathbb{P}}^{\mathfrak{M}} \varphi$ для любого $p \in \mathbb{P}$. Отношение $\Vdash_{\mathbb{P}}$ понимается как $\vdash_{\mathbb{P}}^{\mathbf{V}}$ (т.е. исходной моделью является универсум $\mathbf{V}$ всех множеств).

Теперь о сушествовании генерических множеств. Легко доказать, что $\mathbb{P}$-генерические над $\mathfrak{M}$ множества сушествуют, когда $\mathfrak{M}$ - счетная (транзитивная) модель ZFC. Это, однако, не всегда удобно: например, во многих рассуждениях желательно рассматривать генерические расширени классов вида $\mathbf{L}[a]$, или вообше расширения универсума всех множеств V. В этом случае можно использовать булевозначные расширения $\mathbf{V}^{\mathbb{P}}$ универсума $\mathbf{V}$ (см. о булевозначных моделях в [29], [70]) или же следующий, в сушности, равносильньй принцип.

ТеОрема 4.4. Пусть $\mathbb{P}-$ ЧУ множество в универсуме всех множеств $\mathbf{V}$. Предположение о том, что, в некотором "виртуальном" более широком универсуме $\mathbf{V}^{+}$(например, вида $\mathbf{V}^{\mathbb{P}}$ ), для всякого $p \in \mathbb{P}$ имеется $\mathbb{P}$-генерическое над $\mathbf{V}$ множсество $G \subseteq \mathbb{P}$, содержащее $p$, не приводит ни к каким противоречиям или необоснованным выводам.

4Б. Если конструктивных точек мало, то П 1 -множества имеют свойство совершенного ядра. Импликация справа налево в теореме 3.3 есть простое следствие леммы 4.5: в самом деле, если каждое множество вида $\mathbf{L}[a] \cap \mathbb{N} \omega, a \in \mathbb{N}^{\omega}$, счетно, то, по лемме, любое $\boldsymbol{\Pi}_{1}^{1}$-множество без совершенного ядра также счетно.

ЛЕмма 4.5 (Соловей [88], Любецкий [66]). Если $a \in \mathbb{R} \quad u \quad \Sigma_{2}^{1}(a)$-множсество $X \subseteq \mathbb{N}^{\omega}$ не содержит совериенных подмножеств, то $X \subseteq \mathbf{L}[a]$. 
ДокАЗАТЕльство. По теореме униформизации 1.9, лемму достаточно доказать для $\Pi_{1}^{1}(a)$-множеств (см. конец доказательства в п. 3В). Теперь рассмотрим $\Pi_{1}^{1}(a)$ множество $X \subseteq \mathbb{N}^{\omega}$. Согласно теореме 1.6, мы имеем $X=E(R)=\bigcup_{\xi<\omega_{1}} E_{\xi}(R)$ для подходяшего решета $R=\left\{R_{q}\right\}_{q \in \mathbb{Q}}$ над $\mathbb{N}^{\omega}$ класса $\Delta_{1}^{1}(a)$ (даже $\Delta_{1}^{0}(a)$, но это нам не нужно). Таким образом, нужно проверить, что $E_{\xi}(R) \subseteq \mathbf{L}[a], \forall \xi$. Отметим, что множество $E_{\xi}(R)$ не более чем счетно: в самом деле, оно борелевское и, по предположению, не имеет совершенного ядра.

Случай 1: $\xi<\omega_{1}^{\mathbf{L}[a]}$. Тогда найдется $w \in \mathbf{W O}_{\xi} \cap \mathbf{L}[a]$. Утверждение " $E_{\xi}(R)$ не более чем счетно" может быть выражено следуюшей формулой:

$$
\exists z \forall x \exists n\left(\sigma(w, x) \Longrightarrow x=(z)_{n}\right),
$$

где $\sigma$ есть $\Sigma_{1}^{1}(a)$-формула, даваемая предложением 1.11(iii). Но выделенная формула является, очевидно, $\Sigma_{2}^{1}$-формулой с параметрами $a, w \in \mathbf{L}[a]$, так что по теореме абсолютности она верна и в $\mathbf{L}[a]$, т.е. найдется $z \in \mathbf{L}[a] \cap \mathbb{N} \omega$ такое, что в $\mathbf{L}[a]$ истинно $\forall x \exists n\left(\sigma(w, x) \Longrightarrow x=(z)_{n}\right)$. Снова по абсолютности, последняя формула истинна и в универсуме всех множеств, что означает $E_{\xi}(R) \subseteq\left\{(z)_{n}: n \in \mathbb{N}\right\} \subseteq \mathbf{L}[a]$.

Случай 2: $\omega_{1}^{\mathbf{L}[a]} \leqslant \xi<\omega_{1}$. Здесь мы собираемся доказать, что $E_{\xi}(R)=\varnothing$. Предположим противное: конституанта $E_{\xi}(R)$ непуста. Как и вьше, если $w \in \mathbf{W O}_{\xi}$, то множество $E_{\xi}(R)$ принадлежит $\mathbf{L}[a, w]$ и счетно в $\mathbf{L}[a, w]$.

Рассмотрим форсинг $\mathbb{C}(\xi)=\operatorname{Coll}(\mathbb{N}, \xi)$, предназначенный для "склейки” $\xi$. Таким образом, $\mathbb{C}(\xi)$ состоит из всех конечных последовательностей ординалов $<\xi$, иными словами, всех функций $p: m \rightarrow \xi$, где $m=\{0,1, \ldots, m-1\}$, и $p \leqslant q$ (т.е. $p$ "сильнее"), когда $q \subseteq p$, т.е. $p$ продолжает $q$ как функция. Всякое $\mathbb{C}(\xi)$-генерическое множество $G \subseteq \mathbb{C}(\xi)$ производит функцию $f[G]=\bigcup G: \mathbb{N} \stackrel{\text { на }}{\longrightarrow} \xi-$ генерическую склейку $\xi$. Обратно, мы имеем $G=\{f[G]\lceil n: n \in \mathbb{N}\}$.

Согласно теореме 4.4 , можно рассмотреть $\mathbb{C}(\xi) \times \mathbb{C}(\xi)$-генерическое расширение $\mathbf{V}\left[G, G^{\prime}\right]$ универсума $\mathbf{V}$, порожденное генерической над $\mathbf{V}$ (следовательно, и над $\mathbf{L}[a]$ ) парой множеств $G_{1}, G_{2} \subseteq \mathbb{C}(\xi)$, в котором, по предыдущему, сушествуют две склеиваюшие функции, $f_{1}=\bigcup G_{1}$ и $f_{2}=\bigcup G_{2}$, из $\mathbb{N}$ на $\xi$. Соответственно, имеются и коды $w_{1} \in \mathbf{W O}_{\xi} \cap \mathbf{L}\left[f_{1}\right]$ и $w_{2} \in \mathbf{W O}_{\xi} \cap \mathbf{L}\left[f_{2}\right]$ ординала $\xi$. Согласно доказанному выше, имеем $E_{\xi}(R) \subseteq \mathbf{L}\left[a, f_{1}\right] \cap \mathbf{L}\left[a, f_{2}\right]=\mathbf{L}\left[a, G_{1}\right] \cap \mathbf{L}\left[a, G_{2}\right]$.

Поскольку множества $G_{1}, G_{2}$ образуют генерическую над $\mathbf{L}[a]$ пару, $E_{\xi}(R) \subseteq \mathbf{L}[a]$ по теореме 4.2(iii). Другими словами, раз конституанта $E_{\xi}(R)$ непуста, то найдется $x \in E_{\xi}(R) \cap \mathbf{L}[a]$. Но в этом случае, очевидно, $\xi<\omega_{1}^{\mathbf{L}[x]} \leqslant \omega_{1}^{\mathbf{L}[a]}$, противоречие.

$\square($ лемма и теорема $3.3(\mathrm{i}))$

ЗАМЕЧАНИЕ 4.6 (Мэнсфйлд [72]). Обрашая лемму 4.5, мы видим, что $\Sigma_{2}^{1}(a)$-множество $X \subseteq \mathbb{N}^{\omega}$ с $X \nsubseteq \mathbf{L}[a]$ обязательно содержит совершенное подмножество $P \subseteq X$. Оказывается, последнее можно выбрать с кодом из $\mathbf{L}[a]$, в том смысле, что найдется совершенное дерево $T \in \mathbf{L}[a], T \subseteq \mathbb{N}<\omega$ (см. обозначения в п. 1 A) такое, что $[T] \subseteq X$. Действительно, из соображений, связанных с теоремой униформизации 1.9 , можно считать, что $X$ есть $\Pi_{1}^{1}(a)$-множество. В этой ситуации формула " $X$ содержит совершенное подмножество вида $[T]$ ” приводится к $\Sigma_{2}^{1}(a)$-виду, т.е. она абсолютна по теореме 2.8, так что найдется совершенное дерево $T \in \mathbf{L}[a], T \subseteq \mathbb{N}<\omega$ такое, что $[T] \subseteq X$ истинно в $\mathbf{L}[a]$. Но формула $[T] \subseteq X$ также абсолютна.

Лемма 4.5 позволяет доказать еще один результат, раскрывающий природу варианта $\mathrm{PK}^{-}\left(\Pi_{1}^{1}(a)\right)$ гипотезы совершенного ядра (п. $\left.3 Ж\right)$. 
СлеДСтвиЕ 4.7. Если $a \in \mathbb{N}^{\omega}$, mo $\mathrm{PK}^{-}\left(\Pi_{1}^{1}(a)\right) \Longleftrightarrow\left(\mathbb{N}^{\omega} \nsubseteq \mathbf{L}[a]\right)$.

ДокАЗАТЕльСтво. Импликация слева направо установлена в лемме 3.12. Для доказательства обратной импликации допустим, что $\mathrm{PK}^{-}\left(\Pi_{1}^{1}(a)\right)$ ложно, т.е. найдется функция $\eta: \mathbb{N}^{\omega} \rightarrow \mathbb{N}^{\omega}$, графиик $P$ которой есть $\Pi_{1}^{1}(a)$-множество без совершенного ядра. Тогда $P \subseteq \mathbf{L}[a]$ по лемме 4.5 , откуда $\mathbb{N}^{\omega}=\operatorname{dom} P \subseteq \mathbf{L}[a]$.

Лемма 4.5 допускает другие интересные варианты и обобщения. Мы видели вьше (случай 2 в доказательстве леммы 4.5), что если $R$ - решето класса $\Delta_{1}^{1}(a), a \in \omega_{1}$, то все непустые конституанты $E_{\xi}(R)$ с $\omega_{1}^{\mathbf{L}[a]} \leqslant \xi<\omega_{1}$ несчетны. Известны и другие похожие результаты о “дальних" конституантах. Например, в указанной ситуации $E_{\xi}(R)$ даже не может быть множеством класса $\mathbf{F}_{\sigma}$, вообще, если $\omega_{\alpha}^{\mathbf{L}[a]} \leqslant \xi<\omega_{1}$ и $E_{\xi}(R) \neq \varnothing$, то $E_{\xi}(R)$ не может быть борелевским множеством уровня $\alpha$ борелевской иерархии, см. [93], [36], [40]. Доказательстваэтих результатов достаточно трудоемки, чтобы приводить их в данной статье, а главное, прямо не связаны с обсуждаемьм здесь кругом вопросов.

4В. Форсинг, порожденный идеалом борелевских множеств. С этого пункта начинается доказательство импликаций $\Longleftarrow в$ теореме 3.9 . Наш план состоит в том, чтобы найти характеризацию случайных точек в терминах форсинга. Чтобы не повторяться, пусть, в этом пункте, $\mathfrak{M}$ - это транзитивная модель ZFC (например, универсум $\mathbf{V}$ всех множеств или класс вида $\mathbf{L}[X]$ ). Среди всего многообразия форсингов $\mathbb{P} \in \mathfrak{M}$ можно выделить те, которые некоторым естественньм образом возникают из $\sigma$-УСА-идеалов.

ОПРЕДЕЛЕНИЕ 4.8. Предположим, что $\mathscr{I}$ является $\mathfrak{M}$-абсолютньм $\sigma$-УСА-идеалом. Борелевский форсинг по модулю $\mathscr{I}$ - это множество

$$
\mathbb{P}_{\mathscr{I}}=\left\{p \in \mathbf{B C} \cap \mathfrak{M}: \boldsymbol{B}_{p} \notin \mathscr{I}\right\}, \text { с порядком: } p \leqslant q, \text { когда } \boldsymbol{B}_{p} \subseteq \boldsymbol{B}_{q} .
$$

Полагаем $\mathbb{P}_{\mathscr{I}}^{\mathfrak{M}}=\mathbb{P}_{\mathscr{I}} \cap \mathfrak{M}^{23}$

Заметим, что “условия" $p, q \in \mathbb{P}_{\mathscr{I}}$ совместны в $\mathbb{P}_{\mathscr{I}}$ (и в $\mathbb{P}_{\mathscr{I}}^{\mathfrak{M}}$ ) тогда и только тогда, когда $\boldsymbol{B}_{p} \cap \boldsymbol{B}_{q} \notin \mathscr{I}$. В самом деле, найдется код $c \in \mathbf{B C} \cap \mathfrak{M}$ такой, что $\boldsymbol{B}_{p} \cap \boldsymbol{B}_{q}=\boldsymbol{B}_{c}$. (Определим сначала коды $p^{\prime}, q^{\prime}$ через $\left(p^{\prime}\right)_{n}=p,\left(q^{\prime}\right)_{n}=q, \forall n$, таким образом, $\boldsymbol{B}_{p^{\prime}}$ и $\boldsymbol{B}_{q^{\prime}}$ суть дополнения, соответственно, $\boldsymbol{B}_{p}$ и $\boldsymbol{B}_{q}$. Теперь определим $c$ так, что $(c)_{0}=p^{\prime}$ и $(c)_{n}=q^{\prime}$ при $n \geqslant 1$.) Теперь, в нетривиальном направлении: если $\boldsymbol{B}_{p} \cap \boldsymbol{B}_{q} \notin \mathscr{I}$, то $c \in \mathbb{P}_{\mathscr{I}}$, и, конечно, $c \leqslant p$ и $c \leqslant q$.

Следуюшая лемма показьвает, что $\mathbb{P}_{\mathscr{I}}^{\mathfrak{M}}$ есть УСА-форсинг в $\mathfrak{M}$.

Лемма 4.9. В условиях определения 4.8, множество $\mathbb{P}_{\mathscr{M}}^{\mathfrak{M}}$ и порядок $\leqslant$ принадлежат $\mathfrak{M}$. Форсинг $\mathbb{P}_{\mathfrak{I}}^{\mathfrak{I}}$ удовлетворяет УСА в $\mathfrak{M}:$ любая антицепь $A \in \mathfrak{M}$, $A \subseteq \mathbb{P}_{\mathscr{I}}^{\mathfrak{M}}$, не более чем счетна в $\mathfrak{M}$.

\footnotetext{
${ }^{23}$ Таким образом, с данным определением форсинг состоит из кодов борелевских множеств, а не самих этих множеств. Если взять сами борелевские множества в качестве “условий”, как это часто делается, то форсинг будет обладать большей наглядностью. Однако при этом приходится иметь дело с тем обстоятельством, что борелевское множество $\boldsymbol{B}_{p}$ зависит от того, в каком универсуме производится операция $\boldsymbol{B}_{p}$ : например, если $p \in \mathfrak{M} \cap \mathbf{B C}$, то $\boldsymbol{B}_{p}$, вообще говоря, отлично от $\left(\boldsymbol{B}_{p}\right)^{\mathfrak{M}}$ (т.е. множества $\boldsymbol{B}_{p}$, определенного в $\mathfrak{M}$ ); на самом деле, $\left(\boldsymbol{B}_{p}\right)^{\mathfrak{M}}=\boldsymbol{B}_{p} \cap \mathfrak{M}$. В общем, работать с кодами нам показалось все же проще.
} 
ДокАЗАТЕльство. $\mathbb{P}_{\mathscr{I}}^{\mathfrak{M}} \in \mathfrak{M}$ элементарно следует из $\mathfrak{M}$-абсолютности $\mathscr{I}$. Далее, соотношение $\boldsymbol{B}_{p} \subseteq \boldsymbol{B}_{q}$ выражается $\Pi_{1}^{1}$-форорулой согласно предложению 1.11(iv), поэтому по теореме абсолютности 2.8 порядок $\leqslant$ на $\mathbb{P}_{\mathscr{I}}^{\mathfrak{M}}$ принадлежит $\mathfrak{M}$. А УСА в $\mathfrak{M}$ следует из свойства $3^{\circ}$ (по отношению к $2^{\circ}$ ) идеала $\mathscr{I}$.

ЛЕмма 4.10. В условиях определения 4.8, пусть множсество $D \in \mathfrak{M}, D \subseteq \mathbb{P}_{\mathscr{I}}^{\mathfrak{M}}$, плотно в $\mathbb{P}_{\mathscr{I}}^{\mathfrak{M}}$. Тогда найдется счетное в $\mathfrak{M}$ множество $A \in \mathfrak{M}, A \subseteq D$, такое,

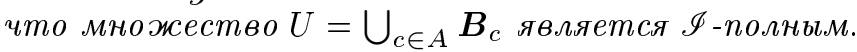

Следовательно, в әтом случае, если $x \in \operatorname{Rand}_{\mathscr{I}} \mathfrak{M}$, то $x \in \bigcup_{c \in D} \boldsymbol{B}_{c}$.

ДокАЗАТЕльство. Выберем, рассуждая в $\mathfrak{M}$, максимальную антицепш $A \subseteq D$, $A \in \mathfrak{M}$. Из леммы 4.9 следует, что $A$ счетна в $\mathfrak{M}$. Для доказательства $\mathscr{I}$-полноты множества $U=\bigcup_{c \in A} \boldsymbol{B}_{c}$, пусть, напротив, $V=\mathbb{N}^{\omega} \backslash U \notin \mathscr{I}$. Из счетности $A$ следует, что $V=\boldsymbol{B}_{p}$ - борелевское множество с кодом $p \in \mathfrak{M}$, значит, $p \in \mathbb{P}_{\mathscr{I}}^{\mathfrak{M}}$. Так как $D$ плотно, найдется "условие" $q \in D, q \leqslant p$. Из-за максимальности $A$, "условие" $q$, следовательно, $p$, совместимо с некоторым $r \in A$, в частности, $\boldsymbol{B}_{p} \cap \boldsymbol{B}_{r} \notin \mathscr{I}$ и $\neq \varnothing$. Но $\boldsymbol{B}_{r} \subseteq U$, противоречие.

Для доказательства последней части леммы остается заметить, что, по выбору $A$, $U$ - борелевское множество с кодом из $\mathbf{B C} \cap \mathfrak{M}$.

Форсинг $\mathbb{P}_{\mathscr{I}}$ делает возможным иной подход к $\mathscr{I}$-случайньм точкам, основанньй на следуюшей лемме.

Лемма 4.11. Если множество $G \subseteq \mathbb{P}_{\mathscr{I}}^{\mathfrak{I}}$ является генерическим над $\mathfrak{M}$, то существует единственная точка $\pi_{G} \in \mathbb{N}^{\omega}$, удовлетворяющая условию с $\in G \Longleftrightarrow$ $\pi_{G} \in \boldsymbol{B}_{c}$ для любого $c \in \mathbf{B C} \cap \mathfrak{M}$.

ДоКАЗАТЕЛЬСТВо. Положим $\boldsymbol{B}_{G}=\left\{\boldsymbol{B}_{p}: p \in G\right\}$; таким образом, $\boldsymbol{B}_{G}$ состоит из самих борелевских множеств, а не их кодов. Тогда любые $X, Y \in \boldsymbol{B}_{G}$ совместны, т.е. найдется $Z \in \boldsymbol{B}_{G}, Z \subseteq X \cap Y$. Множество $D_{n}=\left\{p \in \mathbb{P}: \exists s \in{ }^{n} 2\left(\boldsymbol{B}_{p} \subseteq \mathcal{N}_{s}\right)\right\}$ плотно в $\mathbb{P}$ при любом $n$ благодаря регулярности. Отсюда следует, что для каждого $n$ найдется (единственная благодаря совместности) последовательность $s_{n} \in{ }^{n} 2$ такая, что $\exists p \in G\left(\boldsymbol{B}_{p} \subseteq \mathcal{N}_{s_{n}}\right)$. При этом $s_{n} \subset s_{n+1}, \forall n$, так что сушествует единственная точка $\pi_{G} \in \mathbb{N} \omega$, удовлетворяюшая $\pi_{G}\left\lceil n=s_{n}, \forall n\right.$.

Покажем, что $c \in G \Longleftrightarrow \pi_{G} \in \boldsymbol{B}_{c}, \forall c \in \mathbf{B C} \cap \mathfrak{M}$.

Эта эквивалентность доказьвается индукцией по $\xi$, где $c \in \mathbf{B C}_{\xi}$.

База индукиии: $\xi=0$. Тогда $c=\boldsymbol{k}, k \in \mathbb{N}$, и $\boldsymbol{B}_{c}=\mathscr{N}_{s_{k}}$. Пусть $n=\operatorname{lh} s_{k}$. Вследствие попарной совместности, $c \in G \Longleftrightarrow s_{k}=t_{n}=\pi_{G}\left\lceil n \Longleftrightarrow \pi_{G} \in \boldsymbol{B}_{c}\right.$.

Индуктивный шаг: $0<\xi<\omega_{1}$ и результат получен для всех $c^{\prime} \in \bigcup_{\eta<\xi} \mathbf{B C}_{\eta}$. По определению, $\boldsymbol{B}_{c}=\mathbb{N}^{\omega} \backslash \bigcup_{n} \boldsymbol{B}_{(c)_{n}}$, откуда легко следует, что множество

$$
D_{c}=\left\{p \in \mathbb{P}_{\mathscr{I}}^{\mathfrak{M}}: \boldsymbol{B}_{p} \subseteq \boldsymbol{B}_{c} \vee \exists n\left(\boldsymbol{B}_{p} \subseteq \boldsymbol{B}_{(c)_{n}}\right)\right\}
$$

плотно в $\mathbb{P}_{\mathscr{I}}^{\mathfrak{M}}$. Значит, по генеричности, найдется $p \in G \cap D_{c}$. Таким образом, выполнено $c \in G \Longleftrightarrow \forall n\left((c)_{n} \notin G\right)$, или, по индуктивному предположению, $c \in G \Longleftrightarrow$ $\forall n\left(\pi_{G} \notin \boldsymbol{B}_{(c)_{n}}\right)$. Но правая часть равносильна $\pi_{G} \in \boldsymbol{B}_{c}$.

Точки вида $\pi_{G}$, полученные указанньм способом из $\mathbb{P}_{\mathscr{I}}^{\mathfrak{M}}$-генерических множеств $G$, называются $\mathbb{P}_{\mathscr{I}}^{\mathfrak{M}}$-генерическими (над $\left.\mathfrak{M}\right)$. 
Через $`\langle x, y\rangle$ условимся обозначать каноническое $\mathbb{P}_{\mathscr{I}}^{\mathfrak{M}}$-имя $\breve{z}$ пары $z=\langle x, y\rangle$ (к сожалению, оно отлично от $\langle\breve{x}, \breve{y}\rangle)$. Каноническим именем для $\mathbb{P}_{\mathscr{I}}^{\mathfrak{M}}$-генерической точки называется $\mathbb{P}_{\mathscr{I}}^{\mathfrak{M}}$-имя

$$
\underline{\pi}=\left\{\left\langle p,{ }^{\cup}\langle n, j\rangle\right\rangle: p \in \mathbb{P}_{\mathscr{I}}^{\mathfrak{M}} \wedge n, j \in \mathbb{N} \wedge \forall y \in \boldsymbol{B}_{p}(y(n)=j)\right\} \in \mathfrak{M}
$$

(которое, конечно, зависит от $\mathscr{I}$ и $\mathfrak{M}$, но эту зависимость мы не будем явно указывать, поскольку из контекста всегда будет ясно, какой идеал и какая исходная модель рассматриваются). Тогда $\pi_{G}=\underline{\pi}[G]$ для любого генерического над $\mathfrak{M}$ множества $G \subseteq \mathbb{P}_{\mathscr{I}}^{\mathfrak{M}}$. Таким образом, согласно лемме 4.11 , в условиях определения $4.8, \pi_{G}$ - единственная точка пересечения $\bigcap_{p \in G} \boldsymbol{B}_{p}=\bigcap \boldsymbol{B}_{G}$ и $\boldsymbol{B}_{G}=\left\{c \in \mathbf{B C} \cap \mathbf{L}[a]: \pi_{G} \in \boldsymbol{B}_{c}\right\}$. Отсюда вытекает, что $\mathfrak{M}[G]=\mathfrak{M}\left[\pi_{G}\right]$.

Лемма 4.12. Точки, $\mathbb{P}_{\mathscr{I}}^{\mathfrak{M}}$-генерические над $\mathfrak{M}$, - это в точности әлементы $\operatorname{Rand}_{\mathscr{I}} \mathfrak{M}$. Следовательно, если $x \in \operatorname{Rand}_{\mathscr{I}} \mathfrak{M}$, mо $\omega_{1}^{\mathfrak{M}[x]}=\omega_{1}^{\mathfrak{M}}$.

ДокаЗАтельство. Рассмотрим генерическое над $\mathfrak{M}$ множество $G \subseteq \mathbb{P}_{\mathscr{I}}^{\mathfrak{M}}$. Если $c \in \mathbf{B C}$ и $B_{c} \in \mathscr{I}$, то $c \notin G$, следовательно, $\pi_{G} \notin \boldsymbol{B}_{c}$ по лемме 4.11. Поэтому $\pi_{G} \in$ $\operatorname{Rand}_{\mathscr{I}} \mathfrak{M}$. Обратно, если $x \in \boldsymbol{R a n d}_{\mathscr{I}} \mathfrak{M}$, то множество $G_{x}=\{c \in \mathbf{B C} \cap \mathbf{L}[a]$ : $\left.x \in \boldsymbol{B}_{c}\right\}$ является $\mathbb{P}_{\mathscr{I}}^{\mathfrak{M}}$-генерическим над $\mathfrak{M}$ согласно лемме 4.10. ( $G_{x} \subseteq \mathbb{P}_{\mathscr{I}}^{\mathfrak{M}}$ следует прямо из выбора $x$.) Последняя часть леммы следует из теоремы $4.2(\mathrm{i})$ и леммы 4.9.

Напомним, что, по теореме 4.3(ii), форсинг связан с истинностью в генерических расширениях. В нашем случае оказьвается, что форсинг достаточно простых формул связан с истинностью также и в исходной модели:

ЛЕмма 4.13. Предположим, что $\varphi(x)-\Sigma_{1}^{1}-$ или $\Pi_{1}^{1}$-формула с параметрами из $\mathfrak{M} \cap \mathbb{N}^{\omega}$, а ч̆ получается из $\varphi$ заменой кажсдого параметра $z \in \mathbb{N}^{\omega}$ на $\mathbb{P}_{\mathscr{I}}^{\mathfrak{M}}-$ имя $\breve{z}$. Пусть также $p \in \mathbb{P}_{\mathscr{I}}^{\mathfrak{M}}$. Тогда $p \Vdash_{\mathbb{P}_{\mathscr{I}}^{\mathfrak{M}}}^{\mathfrak{M}} \breve{\varphi}(\underline{\pi})$ равносильно тому, что, в $\mathfrak{M}$, множество $X=\left\{x \in \boldsymbol{B}_{p}: \neg \varphi(x)\right\}$ является $\mathscr{I}$-нулевим.

ДокАЗАТЕЛЬСТво. Если $X$ является $\mathscr{I}$-нулевым, то пусть $c \in \mathbf{B C} \cap \mathfrak{M}$ таково, что $\boldsymbol{B}_{c} \in \mathscr{I}_{\text {и }} X \subseteq \boldsymbol{B}_{c}$ в $\mathfrak{M}$. По выбору $\varphi$ (также используется предложение 1.11(iv)), соотношение $X \subseteq \boldsymbol{B}_{c}$ выражается формулой типа не вьше $\Pi_{2}^{1}$, поэтому по теореме абсолютности 2.8 мы имеем, в любом расширении $\mathfrak{M}, x \notin \boldsymbol{B}_{c} \Longrightarrow \varphi(x)$ для всех $x \in \boldsymbol{B}_{p}$. Но $\pi_{G} \in \boldsymbol{B}_{p}$ для любого генерического $G \subseteq \mathbb{P}_{\mathscr{I}}^{\mathfrak{M}}$, содержащего $p$.

Обратно, пусть $X$ не является $\mathscr{I}$-нулевым. Тогда, по теореме 3.7 , найдется $q \in$ $\mathbf{B C} \cap \mathfrak{M}$ такое, что $\boldsymbol{B}_{q} \notin \mathscr{I}$ и $\boldsymbol{B}_{q} \subseteq X$ в $\mathfrak{M}$. Тогда $q \in \mathbb{P}_{\mathscr{I}}^{\mathfrak{M}}$ и $q \leqslant p$. Более того, вследствие тех же рассуждений, что и вьше, $q$ вынуждает $\neg \breve{\varphi}(\underline{\pi})$, так что $p$ не может вьнуждать $\breve{\varphi}(\underline{\pi})$.

4Г. Два примера. Взяв в качестве $\mathscr{I}$ идеал $\mathscr{I}_{\text {cat }}$ тощих борелевских множеств или идеал $\mathscr{I}_{\boldsymbol{\lambda}}$ борелевских множеств $\boldsymbol{\lambda}$-меры 0 (о других примерах будет сказано в п. 9В), мы получаем два специальных форсинга, связанных с проблемами свойства Бэра и измеримости:

форсинг Коэна: $\mathbb{P}_{\text {соһ }}=\mathbb{P}_{\mathscr{I}_{\text {cat }}}=\left\{p \in \mathbf{B C} \cap \mathfrak{M}: \boldsymbol{B}_{p}-\right.$ не тошее множество $\}$, случайный форсинг ${ }^{24}: \mathbb{P}_{\boldsymbol{\lambda}}=\mathbb{P}_{\mathscr{I}_{\boldsymbol{\lambda}}}=\left\{p \in \mathbf{B C}: \boldsymbol{\lambda}\left(\boldsymbol{B}_{p}\right)>0\right\}$.

Соответственно, $\mathbb{P}_{\text {coh }}^{\mathfrak{M}}=\mathbb{P}_{\text {coh }} \cap \mathfrak{M}=\mathbb{P}_{\mathscr{I}_{\text {cat }}}^{\mathfrak{M}} \mathbb{P}_{\boldsymbol{\lambda}}^{\mathfrak{M}}=\mathbb{P}_{\boldsymbol{\lambda}} \cap \mathfrak{M}=\mathbb{P}_{\mathscr{I}_{\boldsymbol{\lambda}}}^{\mathfrak{M}}$. Напомним, что $\boldsymbol{\lambda}$ - мера на $\mathbb{N}^{\omega}$ (фактически на $2^{\omega}$ ), введенная в п. 1Б.

\footnotetext{
${ }^{24}$ Также называемьй форсингом Соловея.
} 
СлЕДСТВИЕ 4.14. Coh $\mathfrak{M}=\left\{\right.$ все $\mathbb{P}_{\operatorname{Mh}}^{\mathfrak{M}}$-генерические над $\mathfrak{M}$ точки $\}$. Rand $\mathfrak{M}=$ $\left\{\right.$ все $\mathbb{P}_{\boldsymbol{\lambda}}^{\mathfrak{M}}$-генерические над $\mathfrak{M}$ точки\}.

Уместно сказать, что форсинг Коэна обычно ассоциируется с другим ЧУ множеством, а именно, множеством $\mathbf{C}=\mathbb{N}^{<\omega}$, с порядком $s \leqslant t(s$ сильнее) когда $t \subseteq s$.

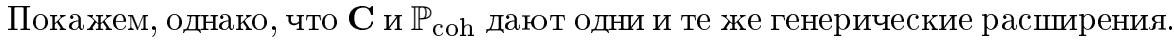

Нетрудно определить рекурсивную функцию $h:\left(\mathbb{N}^{\omega}\right)^{2} \rightarrow \mathbb{N}^{\omega}$ такую, что $h(p, q) \in$ $\mathbf{B C}_{\text {и }} \boldsymbol{B}_{p} \cap \boldsymbol{B}_{q}=\boldsymbol{B}_{h(p, q)}$ всякий раз, когда $p, q \in \mathbf{B C}$. Отсюда следует, согласно предложению 1.11 (vi), что бинарное отношение $p \approx q$, когда симметрическая разность $\boldsymbol{B}_{p} \triangle \boldsymbol{B}_{q}$ есть тощее множество $(p, q \in \mathbf{B C})$, есть $\Sigma_{2}^{1}$, так что его ограничение на $\mathbb{P}_{\text {coh }}^{\mathfrak{M}}$ принадлежит $\mathfrak{M}$. С другой стороны, если $p \approx q$ принадлежат $\mathbb{P}_{\text {coh }}^{\mathfrak{M}}$, то множество

$$
D=\left\{r \in \mathbb{P}_{\mathrm{coh}}^{\mathfrak{M}}: \boldsymbol{B}_{r} \subseteq \boldsymbol{B}_{p} \cap \boldsymbol{B}_{q} \vee \boldsymbol{B}_{r} \cap\left(\boldsymbol{B}_{p} \cup \boldsymbol{B}_{q}\right)=\varnothing\right\}
$$

плотно в $\mathbb{P}_{\text {соһ }}^{\mathfrak{M}}$ (и принадлежит $\left.\mathfrak{M}\right)$, откуда нетрудно вывести, что $p \in G \Longleftrightarrow q \in G$, каково бы ни было $\mathbb{P}_{\text {coh }}^{\mathfrak{M}}$-генерическое над $\mathfrak{M}$ множество $G$. Тем самьм, $\mathbb{P}_{\text {coh }}^{\mathfrak{M}}$-генерические расширения $\mathfrak{M}$ совпадают с $\mathbb{P}$-генерическими, где $\mathbb{P}=\mathbb{P}_{\text {coh }}^{\mathfrak{M}} / \approx$, с порядком $[p]_{\approx} \leqslant[q]_{\approx}$, ког да $\boldsymbol{B}_{p} \backslash \boldsymbol{B}_{q}$ - тощее множество.

Для каждого $s \in 2^{<\omega}=\mathbf{C}$ выберем в $\mathfrak{M}$ код $p_{s} \in \mathbf{B C} \cap \mathfrak{M}$ такой, что $\boldsymbol{B}_{p_{s}}=\mathscr{N}_{s}$. Тогда отображение $s \mapsto\left[p_{s}\right] \approx$ является порядковым изоморфизмом между $\mathbf{C}$ и плотным подмножеством $\mathbb{P}$. (Используется тот факт, что каждое не тошее борелевское $X \subseteq \mathbb{N}^{\omega}$ - котощее хотя бы на одном $\mathscr{N}_{s}$.) Теперь применяем теорему 4.2(ii).

Случайный форсинг также обычно отождествляется с замкнутьми, а не борелевскими множествами положительной меры (см. ниже п. 8Г), но здесь все сразу понятно: любое борелевское множество положительной меры содержит замкнутое подмножество положительной меры.

Отметим, что известный форсинг Сакса [82] также допускает представление в виде $\mathbb{P}_{\mathscr{I}}$, именно, для идеала $\mathscr{I}$ всех не более чем счетных множеств, однако этот идеал не относится, конечно, к $\sigma$-УСА-идеалам.

4Д. Если неслучайных точек мало, то все множества второго проектив-

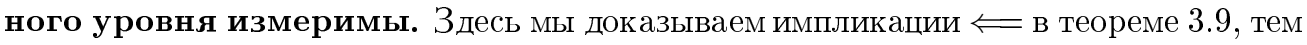
самым, и в эквивалентностях (ii)-(v) теоремы 3.3 и (II)-(V) следствия 3.4 (см. замечание 3.10). Результаты основаны на следующем утверждении.

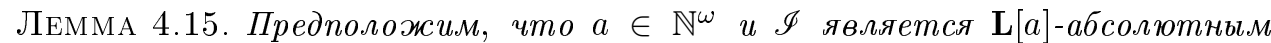
б-УСА-идеалом. Для всякого $\Sigma_{2}^{1}(\mathbf{L}[a])$-множества $X \subseteq \mathbb{N}^{\omega}$ найдется борелевское множсество $U \subseteq \mathbb{N}^{\omega}$ с кодом из $\mathbf{L}[a]$ такое, что $X \cap \operatorname{Rand}_{\mathscr{I}} \mathbf{L}[a]=U \cap$

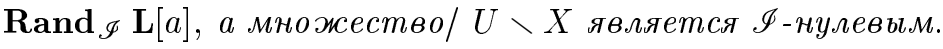

ДоказАТельство. Пусть $X=\left\{x \in \mathbb{N}^{\omega}: \Gamma(x, z)\right\}$, где $z \in \mathbf{L}[a] \cap \mathbb{N}^{\omega}, \Gamma$ есть $\Sigma_{2}^{1}$-формула $\exists y \vartheta(x, z, y)$, а $\vartheta$ - формула типа $\Pi_{1}^{1}$. Мы имеем

$$
x \in X \Longleftrightarrow \text { в } \mathbf{L}[x, a] \text { истинно } \Gamma(x, z) \Longleftrightarrow \exists \xi<\omega_{1}^{\mathbf{L}[a, x]}\left(x \in X_{\xi}\right)
$$

согласно теоремам 2.8 и 2.6(i); здесь $X_{\xi}=\left\{x: \vartheta\left(x, z, f_{\xi}[\tilde{x} a]\right)\right\}$, а $\tilde{x} a$ обозначает “сшивку" $x$ и $a$, т.е. $(\tilde{x} a)(2 k)=x(k)$ и $(\tilde{x} a)(2 k+1)=a(k)$. Равенство $\omega_{1}^{\mathbf{L}[a, x]}=\omega_{1}^{\mathbf{L}[a]}$ леммы 4.12 позволяет улучшить этот результат:

$$
\text { для всех } x \in \mathbf{R a n d}_{\mathscr{I}} \mathbf{L}[a]: \quad x \in X \Longleftrightarrow \exists \xi<\omega_{1}^{\mathbf{L}[a]}\left(x \in X_{\xi}\right) .
$$


Обозначим через $\gamma(w, x, a, z)$ формулу $\forall y(\varphi(w, x \sim a, y) \Longrightarrow \vartheta(x, z, y))$, где $\varphi$ есть $\Sigma_{1}^{1}$-формула, даваемая теоремой 2.6(ii); таким образом, мы имеем $X_{\xi}=\{x$ : $\gamma(w, x, a, z)\}$ всякий раз, когда $w \in \mathbf{W O}_{\xi}$. Положим $X_{\xi}^{\prime}=X_{\xi} \cap \mathbf{L}[a]$.

Рассуждаем в $\mathbf{L}[a]$. Определим $C^{\prime}$ и $\mathscr{I}^{\prime}$, как в $3^{\circ}$ п. 3 Д при $\mathfrak{M}=\mathbf{L}[a]$. По теореме абсолютности 2.8, имеем $X_{\xi}^{\prime}=\{x: \gamma(w, x, a, z)\}$ (в $\left.\mathbf{L}[a]\right)$ всякий раз, когда $\xi<\omega_{1}$ и $w \in \mathbf{W O}_{\xi}$, следовательно, все $X_{\xi}^{\prime}-$ множества класса $\boldsymbol{\Pi}_{1}^{1}$. Значит, по теореме 3.7 , каждое $X_{\xi}^{\prime} \mathscr{I}^{\prime}$-измеримо, т.е. существуют коды $c_{\xi} \in \mathbf{B C}$ и $d_{\xi} \in C^{\prime}$ такие, что $X_{\xi}^{\prime} \triangle \boldsymbol{B}_{c_{\xi}} \subseteq \boldsymbol{B}_{d_{\xi}}$, другими словами, $\boldsymbol{B}_{c_{\xi}} \backslash \boldsymbol{B}_{d_{\xi}} \subseteq X_{\xi}^{\prime} \subseteq \boldsymbol{B}_{c_{\xi}} \cup \boldsymbol{B}_{d_{\xi}}$.

Однако $\mathscr{I}^{\prime}$ удовлетворяет $2^{\circ}$ в $\mathbf{L}[a]$ (свойство $3^{\circ}$ идеала $\mathscr{I}$ ), откуда следует, что найдется такой ординал $\vartheta<\omega_{1}^{\mathrm{L}[a]}$, что $\boldsymbol{B}_{c_{\xi}} \backslash\left(\bigcup_{\eta<\vartheta} \boldsymbol{B}_{c_{\eta}}\right) \in \mathscr{I}^{\prime}$ для всех $\xi \geqslant \vartheta$.

Рассуждаем в универсуме всех множеств. Положим $U_{\xi}=\boldsymbol{B}_{c_{\xi}}$ и $D_{\xi}=\boldsymbol{B}_{d_{\xi}}$. Тогда $D_{\xi} \in \mathscr{I}$, кроме того, применяя теорему абсолютности 2.8 к соответствующим формулам, мы имеем $X_{\xi} \triangle U_{\xi} \subseteq D_{\xi}$ для всех $\xi<\omega_{1}^{\mathbf{L}[a]}$, а также $U_{\xi} \backslash U \in \mathscr{I}$ для всех $\xi \geqslant \vartheta$, где $U=\bigcup_{\eta<\vartheta} U_{\eta}$ - очевидно, борелевское множество с кодом из L $[a]$. По

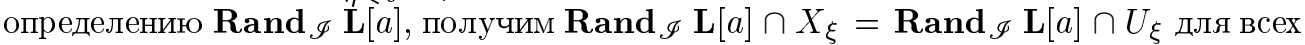
$\xi<\omega_{1}^{\mathbf{L}[a]}$ и $\boldsymbol{R a n d}_{\mathscr{I}} \mathbf{L}[a] \cap U_{\xi} \subseteq U$ при $\vartheta \leqslant \xi<\omega_{1}^{\mathbf{L}[a]}$. Теперь равенство $(X \triangle U) \cap$ $\operatorname{Rand}_{\mathscr{I}} \mathbf{L}[a]=\varnothing$ следует из (2), а $U \backslash X \subseteq \bigcup_{\eta<\vartheta} U_{\eta} \backslash X_{\eta} \subseteq \bigcup_{\eta<\vartheta} D_{\eta}$, так что $U-$ искомое множество.

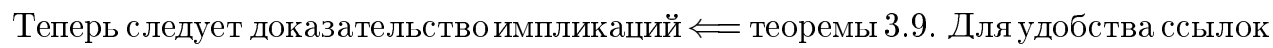
результат формулируется в виде отдельного следствия:

СлЕДСТВИЕ 4.16. (а) В условиях леммы 4.15 если множество $\operatorname{Rand}_{\mathscr{I}} \mathbf{L}[a]$ является $\mathscr{I}$-полным, то все $\Sigma_{2}^{1}(\mathbf{L}[a])$-множества $X \subseteq \mathbb{N} \omega$ I-измеримы.

(б) В условиях леммы 4.15 если $\mathbf{R a n d}_{\mathscr{I}} \mathbf{L}[a] \cap \boldsymbol{B}_{c} \neq \varnothing$ для каждого борелевского $\boldsymbol{B}_{c} \notin \mathscr{I}$ с кодом из $\mathbf{L}[a]$, то все $\Delta_{2}^{1}(\mathbf{L}[a])$-множсества $X \subseteq \mathbb{N}^{\omega} \mathscr{I}$-измеримы.

ДокАЗАТЕЛЬСТвО. (а) Согласно лемме 4.15 , найдется борелевское множество $U$ такое, что $X \cap \boldsymbol{R a n d}_{\mathscr{I}} \mathbf{L}[a]=U \cap \mathbf{R a n d}_{\mathscr{I}} \mathbf{L}[a]$. Eсли $\mathbf{R a n d}_{\mathscr{I}} \mathbf{L}[a]$ является $\mathscr{I}$-полньм, то $X \mathscr{I}$-измеримо, ибо таковым является борелевское $U$.

(б) Рассмотрим пару взаимно дополнительных $\Sigma_{2}^{1}(a)$-множеств $X, Y \subseteq \mathbb{N}^{\omega}$. Лемма 4.15 дает борелевские множества $U, V \subseteq \mathbb{N}^{\omega}$ с кодами из $\mathbf{L}[a]$ такие, что

$X \cap \operatorname{Rand}_{\mathscr{I}} \mathbf{L}[a]=U \cap \operatorname{Rand}_{\mathscr{I}} \mathbf{L}[a] \quad$ и $Y \cap \operatorname{Rand}_{\mathscr{I}} \mathbf{L}[a]=V \cap \operatorname{Rand}_{\mathscr{I}} \mathbf{L}[a]$,

а множества $U \backslash X$ и $V \backslash Y$ являются $\mathscr{I}$-нулевыми. Остается показать, что дополнение $C=\mathbb{N}^{\omega} \backslash(U \cup V)$ множества $U \cup V$ есть $\mathscr{I}$-нулевое множество. Пусть напротив, $C \notin \mathscr{I}$. Понятно, что $C$ - также борелевское множество с кодом из $\mathbf{L}[a]$, следовательно, в наших предположениях, найдется $x \in \mathbf{R a n d}_{\mathscr{I}} \mathbf{L}[a] \cap C$. Коль скоро множества $X, Y$ взаимно дополнительны, $x$ принадлежит одному из них, скажем, $X$. Тогда $x \in U$ по выбору $U$, противоречие.

(теоремы 3.9 и 3.3, следствие 3.4 )

Лемма 4.15, если отвлечься от заключенного в ней требования, чтобы $U \backslash X$ было $\mathscr{I}$-нулевым множеством, допускает далеко идушее обобшение.

ТеОрема 4.17. В условиях леммы 4.15, для всякой $\in$-формульи $\varphi(x)$ с параметрами из $\mathbf{L}[a]$ найдется такой борелевский код $c \in \mathbf{B C} \cap \mathbf{L}[a]$, что әквивалентность “ $\varphi(x)$ истинно в $\mathbf{L}[a, x] \Longleftrightarrow x \in \boldsymbol{B}_{c}$ " выполняется для всех $x \in$ $\operatorname{Rand}_{\mathscr{I}} \mathbf{L}[a]$. 
Другими словами, любое множество вида $X=\left\{x \in \mathbb{N}^{\omega}: \varphi(x)\right.$ истинно в $\left.\mathbf{L}[a, x]\right\}$ (где $\varphi$ имеет параметры только из $\mathbf{L}[a]$ ) совпадает, с точностью до точек, не принадлежаших $\operatorname{Rand}_{\mathscr{I}} \mathbf{L}[a]$, с каким-то борелевским множеством с кодом из $\mathbf{L}[a]$. Это применимо, например, для множеств $X$ класса $\Sigma_{2}^{1}(a)$ или $\Pi_{2}^{1}(a)$, так как (по теореме абсолютности 2.8) соответствуюшие формулы абсолютны для $\mathbf{L}[a]$. О других приложениях этой теоремы будет сказано ниже.

ДокАЗАТЕльство. Считаем, что $\varphi$ есть $\varphi(z, x)$ с единственным параметром $z \in$ $\mathbf{L}[a]$. (Сюда легко сводится случай многих параметров.) Напомним, что $\mathbb{P}_{\mathscr{I}}^{\mathbf{L}[a]}$-имя $\underline{\pi} \in \mathbf{L}[a]$ такое, что $\pi_{G}=\underline{\pi}[G]$, каково бы ни было генерическое множество $G \subseteq \mathbb{P}_{\mathscr{I}}^{\mathbf{L}[a]}$, введено в п. 4В. Отношение $\mathbb{P}_{\mathscr{I}}^{\mathbf{L}[a]}$-вынуждения над $\mathbf{L}[a]$ обозначим через $\Vdash$. Согласно теореме 4.3(iii), множество

$$
D=\left\{p \in \mathbb{P}_{\mathscr{I}}^{\mathbf{L}[a]}: p \Vdash \varphi(\breve{z}, \underline{\pi}) \text { или } p \Vdash \neg \varphi(\breve{z}, \underline{\pi})\right\}
$$

плотно в $\mathbb{P}_{\mathscr{I}}^{\mathbf{L}[a]}$ и принадлежит $\mathbf{L}[a]$. Согласно лемме 4.10 , найдется счетное множество $\left\{p_{n}: n \in \mathbb{N}\right\} \in \mathbf{L}[a]$ условий $p_{n} \in D$ такое, что $\bigcup_{n} \boldsymbol{B}_{p_{n}}-\mathscr{I}$-полное множество. Положим

$$
u=\left\{n: p_{n} \Vdash \varphi(\breve{z}, \underline{\pi})\right\} \quad \text { и } v=\mathbb{N} \backslash u=\left\{n: p_{n} \Vdash \neg \varphi(\breve{z}, \underline{\pi})\right\} .
$$

Ясно, что найдутся борелевские коды $c, c^{\prime} \in \mathbf{B C} \cap \mathbf{L}[a]$ такие, что $\boldsymbol{B}_{c}=\bigcup_{n \in u} \boldsymbol{B}_{p_{n}}$ и $\boldsymbol{B}_{c^{\prime}}=\mathbb{N}^{\omega} \backslash \boldsymbol{B}_{c}=\bigcup_{n \in v} \boldsymbol{B}_{p_{n}}$. Покажем, что $c$ - искомый код. Пусть $x \in \mathbf{R a n d}_{\mathscr{I}} \mathbf{L}[a]$. Тогда $x \in \boldsymbol{B}_{c} \cup \boldsymbol{B}_{c^{\prime}}$. Если $x \in \boldsymbol{B}_{c}$, то $x \in \boldsymbol{B}_{p_{n}}$ для некоторого $n \in u$. С другой стороны, согласно лемме $4.12, x=\pi_{G}$ для некоторого генерического над $\mathbf{L}[a]$ множества $G \subseteq \mathbb{P}_{\mathscr{I}}^{\mathbf{L}[a]} ;$ фактически, $G=G_{x}=\left\{p \in \mathbb{P}_{\mathscr{I}}^{\mathbf{L}[a]}: x \in \boldsymbol{B}_{p}\right\}$. Значит, $p_{n} \in G=G_{x}$, откуда по определению форсинга следует, что формула $\varphi(\breve{z}[G], \underline{\pi}[G])$, т.е. $\varphi(z, x)$, истинна в $\mathbf{L}[a, G]=\mathbf{L}[a, x]$. Если $x \notin \boldsymbol{B}_{c}$, то точно так же $x \in \boldsymbol{B}_{c^{\prime}}$ и формула $\varphi(z, x)$ ложна в $\mathbf{L}[a, x]$.

Исторические и библиографические замечания. Метод вьнуждения (форсинг) Коэна [15] является, пожалуй, наиболее важным методом в теории множеств. Мы рекомендуем [87], [29], [11; гл. 5], а также [10], [44], [30] на английском языке в качестве базовых ссылок, касаюшихся форсинга и теорем 4.1-4.3.

Ссылки по теоремам 3.3 и 3.9 (и следствию 3.4 ), доказательство которых начато в $\S 3$ и закончено в пп. 4Б, 4 Д, приведены в исторических и библиографичиеских замечаниях к $\S 3$.

Для идеала меры 0 и идеала тощих множеств форсинг $\mathbb{P} \mathscr{I}$ был введен Соловеем [89], вместе с конщепщией случайных и коэновских точек и основными конструкциями пा. 4В-4Д, в частности, теоремой 4.17. О равносильности двух определений форсинга Коэна (п. 4Г) см. в [89], однако там использованы другие рассуждения.

\section{$\S$ 5. Комбинаторика финального доминирования}

Содержанием этого короткого параграфа являются результаты, связьваюшие свойства регулярности множеств второго проективного уровня со свойствами одного отношения частичного порядка на $\mathbb{N}^{\omega}$ из группы финальных отношений. Сюда относятся отношения, характеризуемые тем, что некоторое свойство вьполняется для почти всех ${ }^{25}$ значений натурального аргумента.

\footnotetext{
${ }^{25}$ Ниже "почти все" означает: все кроме конечного числа.
} 
Пусть $f, g$ - функщии с $\operatorname{dom} f=\operatorname{dom} g=\omega$. Напомним следуюшие определения.

$f \leqslant{ }^{*} g$ означает, что $f(n) \leqslant g(n)$ для почти всех $n \in \omega$. (Здесь предполагается, что $f, g$ принимают значения в каком-то фиксированном упорядоченном множестве, например, $\mathbb{N}$ или $\mathbb{R}$.)

$f \subseteq^{*} g$ означает, что $f(n) \subseteq g(n)$ для почти всех $n \in \omega$.

$f \in{ }^{*} g$ означает, что $f(n) \in g(n)$ для почти всех $n \in \omega$.

Особый интерес представляет отношение финального доминирования ${ }^{26} \leqslant^{*}$ на множествах вроде $\mathbb{N}^{\omega}$ или $\mathbb{R}^{\omega}$. В частности, любая $\leqslant *$-возрастающая $\omega$-последовательность $\left\{f_{n}\right\}_{n \in \omega}$ элементов $f_{n} \in \mathbb{N}^{\omega} \leqslant{ }^{*}$-ограничена: в самом деле, положим $f(k)=$ $\sup _{n \leqslant k} f_{n}(k)$, тогда $f_{n} \leqslant^{*} f, \forall n$. (Для обычного доминирования, т.е. отношения $f \leqslant g$, когда $f(n) \leqslant g(n)$ для всех $n$, это, конечно, не имеет места.)

В качестве приложения, доказьвается теорема 5.4 о том, что $\operatorname{LM}\left(\Sigma_{2}^{1}\right)$ влечет $\mathrm{BP}\left(\boldsymbol{\Sigma}_{2}^{1}\right)$; впрочем, ее доказательство включает и идеи, связанные с теоремой 3.3. Результаты этого параграфа найдут применение к исследованию свойств регулярности в некоторых сложных генерических моделях в $\S 8$.

5А. Финальное доминирование и измеримость. Здесь мы покажем, как гипотеза $\operatorname{LM}\left(\Sigma_{2}^{1}\right)$ связана со свойствами порядка $\leqslant{ }^{*}$ на множестве

$$
\ell_{1}=\left\{x \in \mathbb{Q}_{+}^{\omega}: \sum_{n \in \omega} x(n)<\infty\right\}
$$

(где $\mathbb{Q}_{+}=\{q \in \mathbb{Q}: q \geqslant 0\}-$ положительные рациональные числа). Мы также рассматриваем совокупность $\mathscr{S}$ всех функщий $\varphi, \omega=\operatorname{dom} \varphi$, принимающих конечные множества в качестве своих значений и удовлетворяющих условию $\sum_{n} \frac{\# \varphi(n)}{(n+1)^{2}}<\infty$, - такие функции будем назьвать медленнымми (т.е. медленно растущими); \#s обозначает число элементов конечного множества $s$.

ТЕОрема 5.1 ([9] или [10; раздел 2.3]). $\operatorname{LM}\left(\Sigma_{2}^{1}\right) \Longleftrightarrow(\mathrm{i}) \Longleftrightarrow$ (ii), где

(i) $\forall a \in \mathbb{N}^{\omega} \quad \exists \varphi \in \mathscr{S} \quad\left(x \in \epsilon^{*} f\right.$ для всех $\left.x \in \mathbf{L}[a] \cap \mathbb{N}^{\omega}\right)$;

(ii) $\forall a \in \mathbb{N}^{\omega} \quad \exists f \in \ell_{1} \quad\left(x \leqslant * f\right.$ для всех $\left.x \in \boldsymbol{\ell}_{1} \cap \mathbf{L}[a]\right)$.

ДокаЗАТЕльство. $\operatorname{LM}\left(\boldsymbol{\Sigma}_{2}^{1}\right) \Longrightarrow(\mathrm{i})$. Фиксируем $a \in \mathbb{N}^{\omega}$. Пусть $\langle i, j\rangle \mapsto n_{i j}-$ peкурсивная биекция $\mathbb{N}^{2}$ на $\mathbb{N}$. Рассмотрим вероятностную меру $\mu$ на $2^{\omega}$, заданную тем, что каждое множество $A_{i j}=\left\{u \in 2^{\omega}: u\left(n_{i j}\right)=1\right\}$ удовлетворяет $\mu\left(A_{i j}\right)=(i+1)^{-2}$ и эти множества в совокупности независимы с точки зрения $\mu$ (т.е., например, $\mu\left(A_{i j} \cap\right.$ $\left.\left.A_{i^{\prime} j^{\prime}} \backslash A_{i^{\prime \prime} j^{\prime \prime}}\right)=\mu\left(A_{i j}\right) \mu\left(A_{i^{\prime} j^{\prime}}\right)\left(1-\mu\left(A_{i^{\prime \prime} j^{\prime \prime}}\right)\right)\right)$. Положим $G_{x}=\lim \sup _{i \rightarrow \infty} A_{i, x(i)}{ }^{27}$ для каждого $x \in \mathbb{N}^{\omega}$. Коль скоро $\sum_{i}(i+1)^{-2}<\infty$, мы имеем $\mu\left(G_{x}\right)=0$ (лемма Бореля-Кантелли).

Из предположения $\operatorname{LM}\left(\boldsymbol{\Sigma}_{2}^{1}\right)$ следует, по теореме 3.3 , что $\boldsymbol{\lambda}(\boldsymbol{R} \operatorname{and} \mathbf{L}[a])=1$. Отсюда $\mu\left(\mathbf{R a n d}_{\mu} \mathbf{L}[a]\right)=1$ (лемма $\left.3.2(3)\right)$. Значит, найдется замкнутое множество $B=$ $[T] \subseteq 2^{\omega}$ положительной $\mu$-меры такое, что $B \cap G_{x}=\varnothing$ для всех $x \in \mathbf{L}[a] \cap \mathbb{N}^{\omega}$, где $T \subseteq 2^{<\omega}-$ совершенное дерево. Можно предполагать, что $\mu\left(\mathscr{C}_{s} \cap B\right)>0$ для всех $s \in T$ (напомним, $\mathscr{C}_{s}=\left\{u \in 2^{\omega}: s \subset u\right\}$ - "канторов интервал" в $2^{\omega}$ ).

\footnotetext{
${ }^{26}$ В англоязычных работах, "eventual domination".

${ }^{27}$ Напомним, что $\lim \sup _{n \in \mathbb{N}} X_{n}=\bigcap_{n} \bigcup_{m \geqslant n} X_{m}$.
} 
Утверждается, что множество $\varphi_{s}(i)=\left\{j: B \cap \mathscr{C}_{s} \cap A_{i j}=\varnothing\right\}$ конечно при $s \in T$ и $i \in \mathbb{N}$. В самом деле, если $j \in f_{s}(i)$, то $B \cap \mathscr{C}_{s} \subseteq 2^{\omega} \backslash A_{i j}$, поэтому раз множества $A_{i j}$, $i \in \varphi_{s}(i)$, независимы в смысле меры и имеют каждое одну и ту же меру $(i+1)^{-2}$, мы имели бы $\mu\left(B \cap \mathscr{C}_{s}\right)=0$ при бесконечном $\varphi_{s}(i)$.

Теперь утверждается, что каждое $\varphi_{s}-$ медленное отображение. Для доказательства заметим, что множество $B \cap \mathscr{C}_{s}$ положительной меры не пересекается с $A_{i j}$ при $i \in \mathbb{N}, j \in \varphi_{s}(i)$. Поэтому (опять лемма Бореля-Кантелли!)

$$
\sum_{i \in \mathbb{N}, j \in \varphi_{s}(i)}(i+1)^{-2}=\sum_{i} \frac{\# \varphi_{s}(i)}{(i+1)^{2}}<\infty .
$$

Далее, утверждается, что существует одна функция $\varphi \in \mathscr{S}$ такая, что при любом $s \in T$ мы имеем $\varphi_{s} \subseteq^{*} \varphi$. Действительно, при любом $k$ функция

$$
\varphi_{k}(i)=\bigcup_{s \in T, \operatorname{lh} s \leqslant k} \varphi_{s}(i)
$$

очевидно, медленная вместе со всеми $\varphi_{s}$. Поэтому существует возрастающая последовательность чисел $m_{0}<m_{1}<m_{2}<\cdots$ такая, что выполнено

$$
\sum_{m_{k} \leqslant i<m_{k+1}} \frac{1}{\# \varphi_{k}(i)} \geqslant 1
$$

Остается определить $\varphi(i)=\varphi_{k}(i)$ при $m_{k} \leqslant i<m_{k+1}$.

Покажем, что $\varphi$ - искомая функция. Пусть $x \in \mathbf{L}[a] \cap \mathbb{N}^{\omega}$. Поскольку $B \cap G_{x}=\varnothing$, найдутся $s \in T$ и $i_{0}$ такие, что $B \cap \mathscr{C}_{s} \cap\left(\bigcup_{i \geqslant i_{0}} A_{i, x(i)}\right)=\varnothing$. Это означает, что $x(i) \in \varphi_{s}(i)$ при $i \geqslant i_{0}$, так что $x \in^{*} \varphi_{s} \subseteq^{*} \varphi$, что и требовалось.

(i) $\Longrightarrow$ (ii). Фиксируем $a \in \mathbb{N}^{\omega}$. Для каждого $f \in \ell_{1}$ существует функция $y_{f} \in$ $\mathbf{L}[a] \cap \mathbb{N}^{\omega}$ такая, что $\sum_{k \geqslant y_{f}(n)} f(n) \leqslant 2^{-n}$. Выберем $\varphi$ согласно (i), а затем положим $y(n)=\sup \varphi(n)$ - тогда $y \in \mathbb{N}^{\omega}$ и $y_{f} \leqslant *$ для всех $f \in \boldsymbol{\ell}_{1} \cap \mathbf{L}[a]$.

Теперь, для $f \in \ell_{1} \cap \mathbf{L}[a]$, определим $f^{\prime}(n)=f\left\lceil[y(n), y(n+1))\right.$, так что $f^{\prime}(n)$ - конечна последовательность рациональных чисел. Тем самьм, применяя (i) к множеству $\boldsymbol{\ell}_{1} \cap \mathbf{L}[a, y]$, мы находим $\varphi \in \mathscr{S}$ такую, что $f^{\prime} \in^{*} \varphi$ для любой $f \in \boldsymbol{\ell}_{1} \cap \mathbf{L}[a]$. Тогда $\sum_{n} \frac{\# \varphi(n)}{(n+1)^{2}}<\infty$, так что можно считать, что \# $\varphi(n) \leqslant(n+1)^{2}$ для всех $n$. Более того, учитьвая вид функций $f^{\prime}$, можно считать, что $\varphi(n)$ состоит из функций $s:[y(n), y(n+)) \rightarrow \mathbb{Q}_{+}$таких, что $\sum_{y(n) \leqslant i<y(n+1)} s(n) \leqslant 2^{-n}$.

Положим $h(i)=\sup _{s \in \varphi(n)} s(i)$ при $y(n) \leqslant i<y(n+1)$. В наших предположениях $\sum_{i} h(i) \leqslant \sum_{n} \sum_{s \in \varphi(n)} \sum_{y(n) \leqslant i<y(n+1)} s(n) \leqslant \sum_{n} n^{2} 2^{-n}<\infty$, так что $h \in \boldsymbol{\ell}_{1}$. $\mathrm{C}$ другой стороны, $f \leqslant^{*} h$ для любой функции $f \in \boldsymbol{\ell}_{1} \cap \mathbf{L}[a]$.

(ii) $\Longrightarrow \operatorname{LM}\left(\Sigma_{2}^{1}\right)$. Здесь сначала доказывается лемма.

Лемма 5.2. Если $X \subseteq 2^{\omega}, \lambda(X)=0 u \varepsilon_{n} \in \mathbb{R}, \varepsilon_{n}>0$ для всех $n \in \mathbb{N}$, mo найдетс последовательность открыто-замкнутых множеств $C_{n} \subseteq 2^{\omega}$ такая, что $\boldsymbol{\lambda}\left(C_{n}\right)<\varepsilon_{n}$ u $X \subseteq \lim \sup _{n} C_{n}$. 
ДокАЗАТЕльство. Прежде всего, найдется система канторовых интервалов $I_{k l} \subseteq 2^{\omega}$ с $X \subseteq \bigcap_{k} \bigcup_{l} I_{k l}$ и $\sum_{l} \lambda\left(I_{k l}\right)<\varepsilon_{0} 2^{-k-1}, \forall k$. Перенумеруем интервалы: пусть $\left\{J_{n}: n \in \mathbb{N}\right\}=\left\{I_{k l}: k, l \in \mathbb{N}\right\}$. Тогда $\sum_{n} \lambda\left(J_{n}\right)<\varepsilon_{0}$ и $X \subseteq \lim \sup _{n} J_{n}$.

Теперь положим $k_{n}=\min k: \sum_{m \geqslant k} \lambda\left(J_{m}\right) \leqslant \varepsilon_{n}$ и $C_{n}=\bigcap_{k_{n} \leqslant k<k_{n+1}} J_{k}$ для всех $n$ - это доказывает лемму.

$($ лемма 5.2)

Продолжая доказательство теоремы (импликация (ii) $\Longrightarrow \operatorname{LM}\left(\boldsymbol{\Sigma}_{2}^{1}\right)$ ), мы возьмем произвольное $a \in \mathbb{N}^{\omega}$ и докажем, что $\boldsymbol{\lambda}(\boldsymbol{R} \operatorname{and} \mathbf{L}[a])=1,-$ этого достаточно по теореме 3.3. Пусть $\left\{X_{\xi}: \xi<\omega_{1}\right\}$ - все борелевские множества $\boldsymbol{\lambda}$-меры 0, имеюшие коды в $\mathbf{L}[a]$; требуется доказать, что $\boldsymbol{\lambda}\left(\bigcup_{\xi} X_{\xi}\right)=0$.

Фиксируем рекурсивное перечисление $\left\{C_{n}: n \in \mathbb{N}\right\}$ всех открыто-замкнутых подмножеств $2^{\omega}$. Используя лемму 5.2 в $\mathrm{L}[a]$, мы находим, для всякого $\xi<\omega_{1}$, функцию $x_{\xi} \in \mathbf{L}[a] \cap \mathbb{N}^{\omega}$ такую, что $X_{\xi} \subseteq \lim \sup _{n} C_{x_{\xi}(n)}$ и $\lambda\left(C_{x_{\xi}(n)}\right)<2^{-n}$ для всех $\xi<\omega_{1}$ и $n$. Положим $f_{\xi}(k)=\boldsymbol{\lambda}\left(C_{k}\right)$ в случае, когда $k \in \operatorname{ran} x_{\xi}=\left\{x_{\xi}(n): n \in \mathbb{N}\right\}$, а иначе $f_{\xi}(k)=0$. Тогда все $f_{\xi}$ принадлежат $\boldsymbol{\ell}_{1} \cap \mathbf{L}[a]$, так что, по предположению (iі), найдется функци $f \in \ell_{1}$ такая, что $f_{\xi} \leqslant * f, \forall \xi$.

Положим $K=\left\{k: f(k) \geqslant \boldsymbol{\lambda}\left(C_{k}\right)\right\}$, и пусть $x \in \mathbb{N}^{\omega}$ осуществляет перечисление всех элементов $K$ в порядке возрастания. Мы утверждаем, что

$$
X_{\xi} \subseteq X=\underset{n}{\limsup } C_{x(n)}, \quad \forall \xi, \quad \text { и } \lambda(X)=0 .
$$

Во-первых, $\sum_{n} \boldsymbol{\lambda}\left(C_{x(n)}\right)=\sum_{k \in K} \boldsymbol{\lambda}\left(C_{k}\right) \leqslant \sum_{k} f(k)<\infty$ (поскольку $f \in \boldsymbol{\ell}_{1}$ ), откуда, по лемме Бореля-Кантелли, $\boldsymbol{\lambda}(X)=0$. Далее, если $z \in X_{\xi}$, то $K_{\xi}=\left\{k \in \operatorname{ran} x_{\xi}\right.$ : $\left.z \in C_{k}\right\}$ бесконечно, причем $\boldsymbol{\lambda}\left(C_{k}\right)=f_{\xi}(k) \leqslant f(k)$ для почти всех $k \in K_{\xi}$, так что, по определению $K$, множество $\left\{k \in K: z \in C_{k}\right\}$ бесконечно. Отсюда следует $z \in X$, что и требовалось.

(теорема 5.1)

5Б. Финальное доминирование и свойство Бэра. Из нескольких известных результатов для $\mathrm{BP}\left(\Sigma_{2}^{1}\right)$, аналогичных теореме 5.1 (см. обзор в [10; 2.2 и 2.4]), для нас наиболее полезна следующая теорема $[10 ; 9.3 .3]$, соединяющая в себе несколько отдельных более ранних результатов, в частности, из [96]. Множество $X \subseteq \mathbb{N}^{\omega}$ называется $\leqslant^{*}$-ограниченным, когда найдется $h \in \mathbb{N}^{\omega}$ такая, что $\forall x \in X\left(x \leqslant{ }^{*} h\right)$. (Ограниченность, очевидно, равносильна возможности накрыти $\sigma$-компактным множеством.)

ТеОРема 5.3. Предположим $\mathrm{BP}\left(\boldsymbol{\Delta}_{2}^{1}\right)$. Тогда $\mathrm{BP}\left(\boldsymbol{\Sigma}_{2}^{1}\right)$ равносильно тому, что каждое множество вида $\mathbf{L}[a] \cap \mathbb{N}^{\omega}, a \in \mathbb{N}^{\omega}, \leqslant^{*}$-ограничено в $\mathbb{N}^{\omega}$.

ДокАЗАТЕЛЬСтво. Допустим, что вьполнено $\mathrm{BP}\left(\boldsymbol{\Sigma}_{2}^{1}\right)$, и рассмотрим произвольное $a \in \mathbb{N}^{\omega}$. По теореме 3.3 , Coh $\mathbf{L}[a]$ - котощее множество. Если $f \in \mathbb{N}^{\omega}$, то положим $f^{\prime}(k)=1+\max _{i \leqslant k} f(i), \forall k$, и $\Phi(f)=\left\{x \in \mathbb{N}^{\omega}: x \leqslant f^{*}\right\}$. Последнее множество - тощее при любом $f$ и является борелевским множеством с кодом из $\mathbf{L}[a]$ при $f \in \mathbf{L}[a] \cap \mathbb{N}^{\omega}$, т.е. в этом случае $\Phi(f) \cap \mathbf{C o h} \mathbf{L}[a]=\varnothing$. Значит, найдется $\mathbf{F}_{\sigma}$-множество $Z=\bigcup_{n} Z_{n} \subseteq \mathbb{N}^{\omega}$ такое, что каждое $Z_{n}$ - замкнутое и нигде не плотное множество и $\Phi(f) \subseteq Z$ для всех $f \in \mathbf{L}[a] \cap \mathbb{N} \omega$. Используя это множество, мы определим, индукцией по $n$, число $k_{n} \in \mathbb{N}$ и $s_{n} \in \mathbb{N}<\omega$. 
Положим $k_{0}=0$. Если $k_{n}$ уже построено, то найдется $s \in \mathbb{N}<\omega$ такое, что мы имеем $\mathscr{N}_{t^{\wedge} s} \cap Z_{j}=\varnothing$ всякий раз, когда $j \leqslant n$, а $t \in \mathbb{N}<\omega$ таково, что lh $t=k_{n}$ и $\operatorname{ran} t \subseteq\left[0, k_{n}\right)$. Возьмем в качестве $s_{n}$ одно из таких $s$ и определим

$$
k_{n+1}=k_{n}+1+\operatorname{lh} s_{n}+\max _{i<\operatorname{lh} s_{n}} s_{n}(i)
$$

что заканчивает индуктивньй шаг построения $s_{n}, k_{n}$. Теперь положим $h(n)=$ $\max _{i<\operatorname{lh} s_{n}} s_{n}(i), \forall n$, и докажем, что $x \leqslant{ }^{*} h$ для любого $x \in \mathbf{L}[a] \cap \mathbb{N}^{\omega}$.

Пусть, напротив, множество $A=\{n: x(n)>h(n)\}$ бесконечно.

Пусть $A=\left\{a_{n}: n \in \mathbb{N}\right\}-$ перечисление в порядке возрастания. Положим

$$
z=u_{0} \wedge s_{a_{0}} \wedge u_{1} \wedge s_{a_{1}} \wedge \ldots \wedge u_{n} \wedge s_{a_{n}} \ldots
$$

где $u_{n}$ - последовательность из $k_{a_{n}}-\sum_{j<n} k_{a_{j}}-\sum_{j<n} \operatorname{lh} s_{a_{j}}$ нулей, так что перед каждым блоком $s_{a_{n}}$ всегда стоит ровно $k_{a_{n}}$ чисел. Отсюда следует, по определению $s_{n}$, что $z \notin Z=\bigcup_{n} Z_{n}$.

Теперь, для получения противоречия, остается доказать, что $z \in \Phi(x)$, другими словами, $z \leqslant^{*} x^{\prime}$. По построению, каждое $z(i)$ есть либо 0 , либо число вида $s_{a_{j}}(l)$, где $k_{a_{j}} \leqslant i$. Во втором случае мы имеем $z(i) \leqslant h\left(a_{j}\right) \leqslant x\left(a_{j}\right) \leqslant x^{\prime}(i)$, что и требовалось.

Обратно, допустим, что $\operatorname{BP}\left(\boldsymbol{\Delta}_{2}^{1}\right)$ и каждое множество вида $\mathbf{L}[a] \cap \mathbb{N}^{\omega}, a \in \mathbb{N}^{\omega}$, $\leqslant^{*}$-ограничено в $\mathbb{N}^{\omega}$. Фиксируем $a \in \mathbb{N}^{\omega}$ и докажем, что множество $\operatorname{Coh} \mathbf{L}[a]$ - котощее; этого достаточно для $\operatorname{LM}\left(\boldsymbol{\Sigma}_{2}^{1}\right)$ согласно следствию 3.4 .

Для начала заметим, что, опять согласно следствию $3.4, \operatorname{Coh} \mathrm{L}[a] \neq \varnothing$; фиксируем произвольное $b \in \mathbf{C o h} \mathbf{L}[a]$. Пусть $\mathscr{T}$ - множество всех деревьев $T \in \mathfrak{M}, T \subseteq \mathbb{N}<\omega$, таких, что $[T]$ - нигде не плотное (замкнутое) подмножество $\mathbb{N}^{\omega}$ и $T$ не имеет $\subseteq$-максимальных элементов. Тогда $b \notin \bigcup_{T \in \mathscr{T}}[T]$, поскольку это объединение совпадает с дополнением Coh L $[a]$. (В самом деле, каждое тошее множество накрьвается счетным объединением нигде не плотных замкнутых множеств, а все фигурируюшие здесь понятия абсолютны по теореме 2.8 и предложению $1.11(\mathrm{vi})$.)

Далее, множество $X$ всех $x \in \mathbb{N}^{\omega}$ таких, что $x(n)=b(n)$ для почти всех $n$, счетно; пусть $X=\left\{x_{n}: n \in \mathbb{N}\right\}$ - перечисление его элементов в $\mathbf{L}[a, b]$. Из сказанного вьше следует, что $X \cap[T]=\varnothing$ для любого $T \in \mathscr{T}$. Через $h_{T}(n)$ обозначим наименьшее $k$ такое, что бэровский интервал $\mathscr{N}_{x_{n} \uparrow k}$ не пересекает $[T]$. Таким образом, $h_{T} \in \mathbf{L}[a, b] \cap \mathbb{N}^{\omega}$. Однако в наших предположениях множество $\mathbf{L}[a, b] \cap \mathbb{N}^{\omega} \leqslant^{*}$-ограничено, т.е. найдется $z \in \mathbb{N} \omega$ такое, что $h_{T} \leqslant{ }^{*} z$ для каждого $T \in \mathscr{T}$. Отсюда следует,

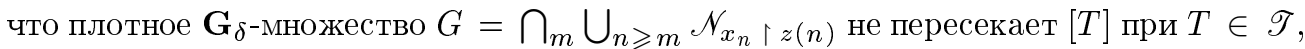
следовательно, включено в $\mathbf{C o h} \mathbf{L}[a]$.

5В. В классе $\Sigma_{2}^{1}$ измеримость влечет свойство Бэра. Здесь доказывается следуюшая теорема.

Теорема 5.4 (Рэзонье и Стерн [80]). $\operatorname{LM}\left(\boldsymbol{\Sigma}_{2}^{1}\right)$ влечет $\operatorname{BP}\left(\boldsymbol{\Sigma}_{2}^{1}\right)$.

Как будет показано ниже, обратная импликация не имеет места, что является наглядным нарушением той дуальности свойств меры и категории, которая прослеживается во многих других случаях. В то же время теорему нельзя рассматривать как связь между мерой вообще и категорией вообще - в самом деле, она не верна, например, для классов $\boldsymbol{\Delta}_{2}^{1}$ и $\boldsymbol{\Delta}_{3}^{1}$ (см. ниже), так что на самом деле речь идет о свойстве, весьма специфическом для класса $\boldsymbol{\Sigma}_{2}^{1}$. 
ДокАЗАТЕльство. Предполагая $\operatorname{LM}\left(\boldsymbol{\Sigma}_{2}^{1}\right)$, фиксируем произвольное $a \in \mathbb{N}^{\omega}$. Тогда, по теореме 3.3 , мы имеем $\boldsymbol{\lambda}(\boldsymbol{R}$ and $\mathbf{L}[a])=1$, а требуется доказать, что $\mathbf{C o h} \mathbf{L}[a]-$ котощее множество, другими словами, что объединение всех нигде не плотных замкнутых подмножеств $\mathbb{N}^{\omega}$ с кодом из $\mathbf{L}[a]$ - тощее множество. В силу того, что $2^{\omega}$ содержит плотное $\mathbf{G}_{\delta}$-множество (с рекурсивным кодом), гомеоморфное $\mathbb{N} \omega$, это утверждение достаточно доказать для пространства $2^{\omega}$ вместо $\mathbb{N}^{\omega}$. Обозначим через $M$ совокупность всех замкнутых нигде не плотных множеств $C \subseteq 2^{\omega}$ с кодом из $\mathbf{L}[a]$. (Под кодом замкнутого $X \subseteq 2^{\omega}$ мы понимаем дерево $T_{X}=\{u \uparrow n: u \in X \wedge n \in \mathbb{N}\} \subseteq 2^{<\omega}$.) Итак, доказывается, что объединение всех множеств $C \in M$ - тощее множество в $2^{\omega}$.

Согласно теореме 5.1 , найдется функция $\varphi \in \mathscr{S}$ такая, что $x \in \in^{*} \varphi$ (т.е. $x(n) \in \varphi(n)$ для почти всех $n$ ) для любого $x \in \mathbf{L}[a] \cap \mathbb{N}^{\omega}$. Мы утверждаем, что $\sum_{n} \frac{1}{\# \varphi(n)}=\infty$. В самом деле, положим $U=\{n: \# \varphi(n) \geqslant n+1\}$, тогда вьполнено неравенство $\sum_{n \in U} \frac{1}{n+1} \leqslant \sum_{n \in U} \frac{\# \varphi(n)}{(n+1)^{2}}<\infty$, следовательно, $\sum_{n \notin U} \frac{1}{n+1}=\infty$, и мы имеем $\sum_{n} \frac{1}{\# \varphi(n)}=\infty$, что и требовалось.

Нашей конечной задачей будет извлечь из $\varphi$ такое плотное $\mathbf{G}_{\delta}$-множество, которое дизъюнктно с каждым $C \in M,-$ что завершает доказательство теоремы. Для этого еще придется немного поработать. Прежде всего, если $C \in M$ и $n \in \mathbb{N}$, то найдутся $k>n$ и функция $\sigma \in{ }^{[n, k)} 2$ такие, что множество $\mathscr{C}_{s} \wedge_{\sigma}=\left\{u \in 2^{\omega}: s^{\wedge} \sigma \subset u\right\}$ не пересекает $C$ при любом $s \in{ }^{n} 2$. (Для пространства $\mathbb{N}^{\omega}$ такое рассуждение не прошло бы!) Обозначим через $k_{C}(n)$ и $\sigma_{C}(n)$ наименьшее $k>n$ и лексикографичиески самую “левую" $\sigma \in{ }^{[n, k)} 2$ указанного вида. Множество $\mathscr{N}_{\sigma_{C}(n)}=\left\{u \in 2^{\omega}: \sigma_{C}(n) \subset u\right\}$ все еще не пересекает $C$.

Ясно, что если $C$ имеет код в $\mathbf{L}[a]$, то $k_{C} \in \mathbf{L}[a]$. Поэтому, по теореме 5.1, найдется функция $\psi \in \mathscr{S}$ такая, что $k_{C} \in^{*} \psi$ для любого $C \in M$. Тогда функция $g(i)=1+\max _{i^{\prime} \leqslant i} \max \psi\left(i^{\prime}\right), \forall i$, удовлетворяет $k_{C} \leqslant{ }^{*} g$ для всех $C \in M$. Заметим, что $g$ - возрастающая положительная функция. Положим $e_{0}=0$ и далее, индукцией по $l, e_{l+1}=g\left(e_{l}\right)$. Пусть теперь $C \in M$. Для всякого $l$, если $e_{l+1} \geqslant k_{C}\left(e_{l}\right)$ (а это будет для почти всех $l$, поскольку $g$ финально доминирует $\left.k_{C}\right)$, то найдется $\tau \in\left[e_{l}, e_{l+1}\right)_{2}$ такая, что $\mathscr{C}_{\tau} \cap C=\varnothing$; обозначим через $\tau_{C}(l)$ самую “левую" $\tau \in\left[e_{l}, e_{l+1}\right) 2$ с таким свойством. Если же $e_{l+1}<k_{C}\left(e_{l}\right)$, то пусть, для определенности, $\tau_{C}(l)$ состоит из одних нулей. Таким образом, $\tau_{C}$ отображает $\mathbb{N}$ в (счетное) множество $\Sigma=\bigcup_{n<k \in \mathbb{N}}{ }^{[n, k)} 2$.

Лемма 5.5. Найдется функиия $h: \omega \rightarrow \Sigma$ такая, что для любого $C \in M$ множество $\left\{l: \tau_{C}(l)=h(l)\right\}$ бесконечно.

ДокАЗАТЕЛЬСтво ЛЕммЫ. Опять по теореме 5.1 , найдется функция $\eta \in \mathscr{S}$ такая, что $\tau_{C} \in^{*} \eta$ всякий раз, когда $C \in M$. Можно считать, что $\eta(l) \subseteq\left[e_{l}, e_{l+1}\right) 2, \forall l$. Снабдим пространство $\Omega=\prod_{l \in \omega} \eta(l)$ мерой $\nu$, которая является произведением однородных вероятностных мер на (конечных) множествах $\eta(l)$. Положим $A_{l}^{\sigma}=\{w \in \Omega$ : $w(l)=\sigma\}$ для $l \in \mathbb{N}$ и $\sigma \in \varphi(l)$. Эти множества, очевидно, независимы в смысле $\nu$, кроме того, если $v \in \Omega$, то $\sum_{l} \nu\left(A_{l}^{v(l)}\right)=\sum_{l} \eta(l)^{-1}=\infty$ (поскольку $\eta \in \mathscr{S}$ ), откуда, опять по лемме Бореля-Кантелли, следует, что множество $X_{v}=\lim \sup _{l \in \mathbb{N}} A_{l}^{v(l)}$ имеет $\nu$-меру 1.

Теперь возьмем такое $a^{\prime} \in \mathbb{N}^{\omega}$, что $a$ (см. начало доказательства теоремы) и $\eta$ принадлежат $\mathbf{L}[a]$. Тогда $\tau_{C} \in \mathbf{L}\left[a^{\prime}\right]$ для любого $C \in M$. В наших предположениях $\operatorname{Rand} \mathbf{L}\left[a^{\prime}\right] \neq \varnothing$, тем самым, и $\boldsymbol{R a n d}_{\nu} \mathbf{L}\left[a^{\prime}\right] \neq \varnothing($ см. лемму 3.2(3)), так что найдется точка $h \in \Omega$, принадлежашая каждому из множеств $X_{v}, v \in \Omega \cap \mathbf{L}$. Это означает, что 
множество $\{l: h(l)=v(l)\}$ бесконечно для каждого такого $v$, т.е., по выбору $\eta, h-$ искомая функция.

Заканчивая доказательство теоремы 5.4, рассмотрим функцию $h$, даваемую леммой. Учитьвая определение $\tau_{C}$, можно считать, что $h(l) \subseteq\left[e_{l}, e_{l+1}\right) 2, \forall l$. Рассмотрим точку $z \in 2^{\omega}$, определенную условием $z\left\lceil\left[e_{l}, e_{l+1}\right)=h(l), \forall l\right.$. Обозначим через $Z=\left\{z_{n}: n \in \mathbb{N}\right\}$ (счетное) множество всех $z^{\prime} \in 2^{\omega} \mathrm{c} z^{\prime}(k)=z(k)$ для почти всех $k$. Тогда, по определению $\tau_{C}$ и $z$ и выбору $h$, мы имеем $Z \cap C=\varnothing$ для любого $C \in M$. Тем самым, для любой пары $C \in M$ и $n$ найдется $j$ такое, что $\mathscr{C}_{z_{n}} \uparrow \cap C=\varnothing$; пусть $j_{C}(n)$ - наименьшее из таких $j$. Тогда каждая из функций $j_{C}, C \in M$, принадлежит $\mathbf{L}[a, z]$, поэтому, как и выше, найдется точка $r \in \mathbb{N} \omega$, финально доминируюшая каждую $j_{C}$, $C \in M$, т.е. $j_{C} \leqslant^{*} r$. Положим $Y_{n}=\mathscr{C}_{z_{n} \uparrow r(n)}$. Тогда $\mathbf{G}_{\delta}$-множество $U=\lim \sup _{n} Y_{n}$ плотно в $2^{\omega}$ (здесь важна плотность $Z$ ) и не пересекает никакое из $C \in M$.

$\square($ теорема 5.4)

Исторические и библиографические замечания. Отношение финального доминирования $\leqslant *$ восходит к старьм работам Дюбуа-Раймона 70-х годов XIX века (см., например, [18]), где оно использовалось как инструмент изучения сравнительной скорости возрастания последовательностей. Хаусдорф доказал для него свою знаменитую теорему о $\left(\omega_{1}, \omega_{1}\right)$-щели в [22] (передоказанную в [24], откуда она, собственно, и стала известна, поскольку работа [22] с очевидностью опередила свое время, и кроме того, появилась в малоизвестном провинциальном журнале).

Начиная с 60-х годов, отношение $\leqslant *$ (и близкие отношения вроде почти дизъюнктности множеств натуральных чисел) стали предметом многочисленных исследований, см., например, обзоры [16], [71].

\section{§ 6. “Элементарное” доказательство одной из теорем}

Вернемся к результатам, показанным нетривиальньми импликациями на диаграмме из введения. Они, несомненно, выражают утверждения чисто дескриптивно-теоретической природы, говоряшие о свойствах точечных множеств (в отличие, скажем, от эквивалентностей теорем 3.3 и 3.9 , где фигурируют понятия, связанные с гёделевой конструктивностью). В то же время рассуждения, которые составляют их доказательство (т.е. изложенные выше доказательства теорем 3.3 и 3.9), апеллируют к объектам (вроде произвольных ординалов и конструктивных множеств) и методам (вроде форсинга), выходящим за рамки круга объектов, естественно рассматриваемых в дескриптивной теории множеств; к последним можно отнести

( ) натуральные и вешественные числа, счетные ординалы, точки из $\mathbb{N} \omega$, вообще польские пространства и их точки, проективные и в особенности борелевские множества в польских пространствах, счетные (и трансфинитные длины $\left.\leqslant \omega_{1}\right)$ “эффективные” последовательности точек и проективных (борелевских) множеств (например, возникаюшие в построении конструктивных множеств до шага $\left.\omega_{1}\right)$, а также простые комбинации этих понятий.

Рассмотрим, например, следующую теорему - одну из двух импликаций диаграммы из введения, выходящую из блока $\operatorname{PK}\left(\mathbf{\Pi}_{1}^{1}\right)^{28}$ :

\footnotetext{
${ }^{28} \mathrm{C}$ небольшими изменениями, которые мы, для экономии места, опускаем, все сказанное ниже относится и ко второй из этих импликаций, т.е. к $\operatorname{PK}\left(\Pi_{1}^{1}\right) \Longrightarrow \operatorname{BP}\left(\Sigma_{2}^{1}\right)$.
} 
Теорема 6.1 (теорема Любецкого). $\operatorname{PK}\left(\boldsymbol{\Pi}_{1}^{1}\right)$ влечет $\operatorname{LM}\left(\boldsymbol{\Sigma}_{2}^{1}\right)$.

Вопрос о том, можно ли доказать эту теорему с использованием только понятий, перечисленных в списке $(\star)$, интересен и важен с позиций философии и методологии математики; в этом смысле его можно сравнить, например, с вопросом о возможности передоказать великую теорему Ферма методами элементарной теории чисел.

Если искать доказательство теоремы 6.1 в еще более ограниченных рамках классической дескриптивной теории множеств, как она сложилась во времена Лузина, то вопрос остается открытым: задача полной элиминашии конструктивности и форсинга из доказательства теоремы 6.1 не решена. Однако менее претенциозная задача модифицировать рассуждения $\S 3$ и $\S 4$, включая используемый там аппарат форсинга, так, чтобы они сушественно не выходили за рамки $(\star)$, решается положительно. Этот параграф̆ посвящен такой модификации. Предлагаемая конструкция представляет собой развитие некоторых идей из [69].

Изложенное ниже доказательство мы называем "элементарньм", беря это слово в кавычки, поскольку элементарность означает здесь ограничение используемых средств доказательства, но не сложности рассуждений. Последняя, на самом деле, даже несколько возрастает, поскольку нам приходится проверять, в новой ситуации, те базовые свойства форсинга, которые в стандартном изложении давно установлены.

6А. Анализ ее доказательства. Мы можем собрать доказательство теоремы 6.1 из следуюших частей, представляющих собой фрагменты доказательства теорем 3.3 и 3.9 в $§ \S 3,4$.

Часть 1: Импликация $\operatorname{PK}\left(\mathbf{\Pi}_{1}^{1}\right) \Longrightarrow \forall a \in \mathbb{N}^{\omega}\left(\mathbf{L}[a] \cap \mathbb{N}^{\omega}\right.$ счетно), которая доказана (в более эффективной форме) в п. 3B.

Часть 2: Импликация $\forall a \in \mathbb{N}^{\omega}\left(\mathbf{L}[a] \cap \mathbb{N}^{\omega}\right.$ счетно $) \Longrightarrow \boldsymbol{\lambda}(\mathbf{R a n d} \mathbf{L}[a])=1$.

Часть 3: Импликация: если $a \in \mathbb{N}^{\omega}$ u $\boldsymbol{\lambda}(\mathbf{R} \operatorname{and} \mathbf{L}[a])=1$, то все $\Sigma_{2}^{1}(a)$-множества $\lambda$-измеримы. Это частный случай $\mathscr{I}=\mathscr{I}_{\boldsymbol{\lambda}}$ следствия 4.16.

Часть 1 в принципе удовлетворяет требованию не выходить в рассуждениях за рамки объектов $(\star)$ (конструктивное построение используется только до шага $\left.\omega_{1}\right)$. Часть 2 вообще тривиальна. О днако часть 3 включает форсинг, которьй, в стандартном изложении существенно выходит за рамки ( $)$, например, в силу того, что он включает рассмотрение собственных классов множеств, в частности, классов вида $\mathbf{L}[a]$ и их генерических расширений. Поэтому нашей задачей станет доказательство следствия 4.16 в рамках объектов, упомянутых в списке $(\star)$.

6Б. Дескриптивные континуумы. Для начала, нам нужен тип структур из (*), которыми можно достаточно адекватно заменить транзитивные модели ZFC (например, классы $\mathbf{L}[a]$ ) в качестве исходных моделей для генерических расширений. Назовем дескриптивным континуумом всякое множество $\mathrm{M} \subseteq \mathbb{N}^{\omega}$ такое, что структура $\langle\mathbb{N} ; \mathrm{M}\rangle$ является $\beta$-моделью арифметики второго порядка. Это включает такие три требования:

Свертка: если $\varphi\left(k, l, x_{1}, \ldots, x_{n}\right)$ - аналитическая формула (как в п. 1В) и $a_{1}, \ldots$, $a_{n} \in \mathrm{M}$, причем $\left\{\langle k, l\rangle: \varphi^{\mathrm{M}}\left(k, l, a_{1}, \ldots, a_{n}\right)\right\}$ - график (всюду определенной) функции $b: \mathbb{N} \rightarrow \mathbb{N}$, то $b \in \mathbf{M}$;

Счетный зависимый выбор: если $\varphi\left(y, z, x_{1}, \ldots, x_{n}\right)$ - аналитическая формула, $a_{1}, \ldots, a_{n} \in \mathrm{M}$ и $\forall y \in \mathrm{M} \exists z \in \mathrm{M} \varphi^{\mathrm{M}}\left(y, z, a_{1}, \ldots, a_{n}\right)$, то найдется $b \in \mathrm{M}$ такое, что вьполнено $\forall k \varphi^{\mathrm{M}}\left((b)_{k},(b)_{k+1}, a_{1}, \ldots, a_{n}\right)$; 
Абсолютность полного упорядочения: если $w \in \mathbf{M} \backslash \mathbf{W O}$, т.е. множество $Q_{w}=\left\{q_{n}: w(n)=0\right\}$ не является вполне упорядоченньм в $\mathbb{Q}$ (см. обозначения примера 1.10.1), то найдется $a \in \mathrm{M}$, кодируюшее убывающую последовательность в $Q_{w}$ в том смысле, что $a(n) \in Q_{w}$ и $q_{a(n+1)}<q_{a(n)}$ для всех $n$.

В этих определениях через $\varphi^{\mathrm{M}}$ обозначена релятивизация формулы $\varphi$ к M, которая означает, что каждый квантор вида $\exists x$ или $\forall x$ (подразумевается: $\exists x \in \mathbb{N}^{\omega}, \forall x \in \mathbb{N}^{\omega}$ ) меняется на, соответственно, $\exists x \in \mathrm{M}, \forall x \in \mathrm{M}$.

Например, дескриптивным континуумом будет само $\mathbb{N}^{\omega}$, а также любое множество вида $\mathfrak{M} \cap \mathbb{N}^{\omega}$, где $\mathfrak{M}$ - любая транзитивная модель теории $\mathbf{Z F C}$, или хотя бы $\mathbf{Z F C}^{-}$(без аксиомы степени). Обратно, если $\mathbf{M} \subseteq \mathbb{N}^{\omega}$ - дескриптивный континуум, то найдется транзитивная модель $\mathfrak{M}$ теории $\mathbf{Z F C}-$ (не обязательно $\mathbf{Z F C}$ ) такая, что $M=\mathfrak{M} \cap \mathbb{N}^{\omega}$. (Этим результатом мы не будем пользоваться.)

Положим hgt $\mathrm{M}=\sup _{w \in \mathrm{M} \cap \mathrm{wo}}|w|($ выссота $\mathrm{M})$ для каждого дескриптивного континуума М, где, напомним, $|w|<\omega_{1}-$ ординал, соответствуюший $w$.

Следуюшая теорема выражает, в сушности, то же самое, что и теорема абсолютности 2.8 , и ее доказательство ничем не отличается. Как обычно, замкнутая аналитическая формула $\Phi$ абсолютна для $\mathrm{M}$, когда $\Phi \Longleftrightarrow \Phi^{\mathrm{M}}$.

ТЕОРема 6.2. Предположим, что $\mathrm{M}$ - дескриптивный континуум. Тогда

(i) всякая $\Sigma_{1}^{1}$-формула $\Phi$ с параметрами из $\mathrm{M}$ абсолютна для $\mathrm{M}$;

(ii) ecлu hgt $\mathrm{M}=\omega_{1}$ (это будет ниже типичньм случаем), то всякая $\Sigma_{1}^{1}$-формула $\Phi$ с параметрами из $\mathrm{M}$ абсолютна для М.

6В. Генерические расширения дескриптивных континуумов. Зафиксируем для дальнейшего $a \in \mathbb{N}^{\omega}$ и $\mathbf{L}[a]$-абсолютный $\sigma$-УСА-идеал $\mathscr{I}$ (см. п. 3Д). Дескриптивный континуум $\mathbf{M}=\mathbf{L}[a] \cap \mathbb{N}^{\omega}$ будет исходной моделью, а в качестве форсинга возьмем $\mathbb{P}_{\mathscr{I}}^{\mathbf{M}}=\mathbb{P}_{\mathscr{I}}^{\mathbf{L}[a]}=\left\{p \in \mathbf{M} \cap \mathbf{B C}: \boldsymbol{B}_{p} \notin \mathscr{I}\right\}$. Желая строить рассуждения как можно ближе к $\mathrm{M}$ и, соответственно, как можно меньше обрашаться к $\mathbf{L}[a]$, мы начнем с "перевода в М-терминологию" того, что определение $\mathbf{L}[a]$-абсолютного $\sigma$-УСА-идеала означает для $\mathbf{L}[a]$.

ПреДЛОЖенИЕ 6.3. Множество $\operatorname{cod} \mathscr{I} \cap \mathrm{M}=\left\{p \in \mathbf{B C} \cap \mathrm{M}: \boldsymbol{B}_{p} \in \mathscr{I}\right\}$ определимо в $\mathrm{M} \Pi_{1}^{1}$-формулой с параметром $а$, а (дополнительное в $\left.\mathrm{BC} \cap \mathrm{M}\right)$ множество $\mathbb{P}_{\mathscr{I}}^{\mathrm{M}}$ определимо в $\mathrm{M} \Sigma_{1}^{1}$-формулой с параметром а.

Если множество $A \subseteq \mathbf{B C} \cap \mathrm{M}$ определимо в $\mathrm{M}$ аналитической формулой $c$ параметрами из $\mathrm{M}$ и $\boldsymbol{B}_{c} \cap \boldsymbol{B}_{c^{\prime}} \in \mathscr{I}$ для $c \neq c^{\prime} \in A$, то $A$ счетно и найдется $z \in \mathrm{M}$ такое, чтол $A=\left\{(z)_{k}: k \in \mathbb{N}\right\}$.

Наконеи (= лемма 4.10), если множество $D \subseteq \mathbb{P}_{\mathscr{I}}^{\mathrm{M}}$ определимо в $\mathrm{M}$ аналитической формулой с параметрами из $\mathrm{M}$, то найдется $c \in \mathrm{M}$ такое, что $A=$ $\left\{(c)_{n}: n \in \mathbb{N}\right\} \subseteq D u \bigcup_{p \in A} \boldsymbol{B}_{p}-\mathscr{I}$-полное множество.

Расширение. Как показано в п. 4B, форсинг $\mathbb{P}_{\mathscr{I}}^{\mathrm{M}}$ естественным образом производит $\mathscr{I}$-случайные точки $x \in \mathbb{N}^{\omega}$ (элементы множества $\operatorname{Rand}_{\mathscr{I}} \mathrm{M}$ ). Как, однако, определить расширение $\mathrm{M}$ посредством $x$ в рамках структуры $\mathbb{N}^{\omega}$ ? Нашей идеей будет определить расширение как совокупность всех точек вида $F(x)$, где $F: \mathbb{N}^{\omega} \rightarrow \mathbb{N}^{\omega}-$ борелевская функция с кодом из М.

Зафиксируем рекурсивньй гомеоморфизм $H:\left(\mathbb{N}^{\omega}\right)^{2} \stackrel{\text { на }}{\longrightarrow} \mathbb{N}^{\omega}$ с парой обратных функций $H_{1}, H_{2}: \mathbb{N}^{\omega} \stackrel{\text { на }}{\longrightarrow} \mathbb{N}^{\omega}$. Положим $\mathrm{F}_{c}=\left\{\left\langle H_{1}(z), H_{2}(z)\right\rangle: z \in \boldsymbol{B}_{c}\right\}$ для каждого 
$c \in \mathbf{B C}$ - это борелевское подмножество $\mathbb{N}^{\omega} \times \mathbb{N}^{\omega}$. Через $\mathbf{B F}$ обозначим множество всех $\tau \in \mathbf{B C}$ таких, что $\mathrm{F}_{\tau}-$ график (борелевской) функции из $\mathbb{N}^{\omega}$ в $\mathbb{N} \omega$. Если $\tau \in \mathbf{B F}$, то определим $\tau^{\prime} x=\mathrm{F}_{\tau}(x)$ для всех $x \in \mathbb{N}^{\omega}$.

Положим $\mathrm{M}\lceil x\rceil=\left\{\tau^{\prime} x: \tau \in \mathbf{M} \cap \mathbf{B F}\right\}^{29}$.

Вряд ли можно утверждать, что М $\lceil x\rceil$ снова будет дескриптивньм континуумом в самом обшем случае, однако это вьполнено в некоторых важных случаях, см., например, теорему 6.6 ниже.

Форсинг. Назовем $\underline{\pi}$-формулой всякую аналитическую формулу (см. п. 1В) $\Phi$ без параметров из $\mathbb{N} \omega$, некоторые или все свободные переменные типа 1 которой заменены выражениями вида $\tau^{\prime} \underline{\pi}$, где $\tau \in \mathbf{B F}$, а $\underline{\pi}-$ просто некоторый символ. В этом случае, если $x \in \mathbb{N}^{\omega}$, то через $\Phi\lceil x\rceil$ будет обозначаться результат замены в $\Phi$ каждого включения вида $\tau^{\prime} \underline{\pi}$ на $\tau^{\prime} x=\mathrm{F}_{\tau}(x)$ - это, очевидно, аналитическая формула с параметрами из $\mathbb{N}^{\omega}$.

Положим $\operatorname{Par} \Phi=\left\{\tau \in \mathbf{B F}: \tau^{\prime} \underline{\pi}\right.$ встречается в $\left.\Phi\right\}$.

Определим бинарное отношение $p \Vdash_{\mathscr{I}}^{\mathrm{M}} \Phi$, где предполагается, что $p \in \mathbb{P}_{\mathscr{I}}^{\mathrm{M}}$, а $\Phi-$

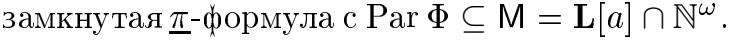

Определение проходит индукцией по сложности $\Phi$ :

(1) если $\Phi-$ ограниченная формула, то $p \Vdash_{\mathscr{I}}^{\mathrm{M}} \Phi \Longleftrightarrow\left\{x \in \boldsymbol{B}_{p}: \neg \Phi\lceil x\rceil\right\} \in \mathscr{I}$;

(2) $p \Vdash_{\mathscr{I}}^{\mathrm{M}} \forall k \Phi(k) \Longleftrightarrow \forall k \in \mathbb{N}\left(p \Vdash_{\mathscr{I}}^{\mathrm{M}} \Phi(k)\right)$;

(3) $p \Vdash \mathbb{I} \exists k \Phi(k) \Longleftrightarrow \forall q \leqslant p \exists r \leqslant q \exists k \in \mathbb{N}(r \Vdash \mathbb{I} \Phi(k))$;

(4) $p \Vdash_{\mathscr{I}}^{\mathrm{M}} \forall x \Phi(x) \Longleftrightarrow \forall \tau \in \mathbf{B F} \cap \mathbf{M}\left(p \vdash_{\mathscr{I}}^{\mathrm{M}} \Phi\left(\tau^{\prime} \underline{\pi}\right)\right)$;

(5) $p \Vdash_{\mathscr{I}}^{\mathrm{M}} \exists x \Phi(x) \Longleftrightarrow \forall q \leqslant p \exists r \leqslant q \exists \tau \in \mathbf{B F} \cap \mathrm{M}\left(r \Vdash_{\mathscr{I}}^{\mathrm{M}} \Phi\left(\tau^{\prime} \underline{\pi}\right)\right)$;

(6) $p \Vdash_{\mathscr{I}}^{\mathrm{M}} \neg \Phi(x) \Longleftrightarrow \forall q \leqslant p \neg\left(q \Vdash_{\mathscr{I}}^{\mathrm{M}} \Phi\right)$.

Переменные $q, r$ имеют область пробегания $\mathbb{P}_{\mathscr{I}}^{\mathrm{M}}$ в $(3),(5),(6)$.

Легко видеть, что определения (3) и (5) могут быть сведены к определениям (2), (4), (6) через замену квантора $\exists$ на $\neg \forall \neg$.

Если $\Phi$ - ограниченная формула, то вынуждение $\Vdash_{\mathscr{I}}^{\mathrm{M}}$ формулы $\neg \Phi$ может быть определено через (1), а может быть сведено к вынуждению $\Phi$ через (6). Однако, как нетрудно проверить, это приносит один и тот же результат.

ТеОрема 6.4. Пусть $\varphi\left(k_{1}, \ldots, k_{j}, x_{1}, \ldots, x_{n}\right)$ - беспараметрическая аналитическая формула с переменными $k_{1}, \ldots, k_{j}, x_{1}, \ldots, x_{n}$. Тогда множество

$$
\begin{gathered}
F_{\varphi}=\left\{\left\langle p, k_{1}, \ldots, k_{j}, \tau_{1}, \ldots, \tau_{n}\right\rangle: k_{1}, \ldots, k_{j} \in \mathbb{N} \wedge \tau_{1}, \ldots, \tau_{n} \in \mathbf{B F} \cap \mathbf{M}\right. \\
\left.\wedge p \in \mathbb{P}_{\mathscr{I}}^{\mathrm{M}} \wedge p \Vdash_{\mathscr{I}}^{\mathrm{M}} \varphi\left(k_{1}, \ldots, k_{j}, \tau_{1}{ }^{\prime} \underline{\pi}, \ldots, \tau_{n}{ }^{\prime} \underline{\pi}\right)\right\}
\end{gathered}
$$

определимо аналитической формулой с параметром а, релятивизованной $к \mathrm{M}$.

ДоКАЗАТЕЛЬСТВО. Используется индукция по сложности $\varphi$. В случае ограниченных формул пусть, для простоты, $\varphi$ есть $\varphi(k, x)$. Мы имеем

$$
F_{\varphi}=\left\{\langle p, k, \tau\rangle: k \in \mathbb{N} \wedge p \in \mathbb{P}_{\mathscr{I}}^{\mathrm{M}} \wedge \tau \in \mathbf{B F} \cap \mathrm{M} \wedge \forall x \in \boldsymbol{B}_{p} \varphi\left(k, \tau^{\prime} x\right)\right\}
$$

согласно определению (1). Однако формула, определяющая это множество, является $\Pi_{2}^{1}$ (это определяется членом $\tau \in \mathbf{B F}$; все остальное еше проше), которая, по теореме 2.8 , абсолютна для $\mathbf{M}=\mathbf{L}[a] \cap \mathbb{N}^{\omega}$.

\footnotetext{
${ }^{29}$ Мыпишем $\lceil x\rceil$ вместо более привьчного $[x]$, чтобы устранить пу таницу с обычной конструкцией генерического расширения.
} 
Индуктивный шаг, разбиваемьй на случаи, соответствующие определениям (2)-(6), является достаточно рутинным рассуждением, основанным на том, что множества $\mathbb{P}_{\mathscr{I}}^{\mathrm{M}}$ и $\mathbf{B F} \cap \mathrm{M}$ определимы в $\mathrm{M}$.

Напомним, что $\mathbf{M}=\mathbf{L}[a] \cap \mathbb{N}^{\omega}$. Положим $\operatorname{Rand}_{\mathscr{I}} \mathbf{M}=\operatorname{Rand}_{\mathscr{I}} \mathbf{L}[a]$.

Теорема 6.5. Предположим, что $x \in \operatorname{Rand}_{\mathscr{I}} \mathrm{M}$, a $\Phi$ - замкнутая $\underline{\pi}$-формула $c \Phi \subseteq$ M. Тогда

$$
\Phi\lceil x\rceil \text { истинно в } \mathrm{M}\lceil x\rceil \Longleftrightarrow \exists p \in \mathbb{P}_{\mathscr{I}}^{\mathrm{M}}\left(x \in \boldsymbol{B}_{p} \wedge p \Vdash_{\mathscr{I}}^{\mathrm{M}} \Phi\right) .
$$

ДоКАЗАТЕЛЬСтво. Рассуждаем индукцией по сложности $\Phi$. Пусть $\Phi$ - ограниченная формула. Если $p \in \mathbb{P}_{\mathscr{I}}^{\mathrm{M}}, x \in \boldsymbol{B}_{p}$ и $p \Vdash_{\mathscr{I}}^{\mathrm{M}} \boldsymbol{\Phi}$, то множество $X=\left\{x^{\prime} \in \boldsymbol{B}_{p}\right.$ : $\left.\neg \Phi\left\lceil x^{\prime}\right\rceil\right\}$ принадлежит $\mathscr{I}$ и является борелевским множеством с кодом из М, так что $x \notin X$ и мы имеем $\Phi\lceil x\rceil$. Но формула $\Phi\lceil x\rceil$ абсолютна. Обратно, если правая часть эквивалентности теоремы не верна, то стандартные рассуждения приносят условие $q \in \mathbb{P}_{\mathscr{I}}^{\mathrm{M}}$ такое, что $x \in \boldsymbol{B}_{q}$ и $q \Vdash_{\mathscr{I}}^{\mathrm{M}} \neg \Phi$. Однако $\neg \Phi-$ опять ограниченная формула, так что по уже доказанному $\Phi\lceil x\rceil$ ложно.

Рассмотрим индуктивный шаг (4). Пусть $\Phi$ есть $\forall y \Psi(y)$. Из определения М $\lceil x\rceil$ следует, что $\Phi\lceil x\rceil$ истинно в $\mathrm{M}\lceil x\rceil$, если и только если $\Psi\left(\tau^{\prime} \underline{\pi}\right)\lceil x\rceil$ истинно в $\mathrm{M}\lceil x\rceil$ при любом $\tau \in \mathbf{B F} \cap \mathbf{M}$. Правая часть (4) имеет аналогичную структуру. Это позволяет свести эквивалентность теоремы для формулы $\Phi$ к индуктивному предположению о ее справедливости для формул вида $\Psi\left(\tau^{\prime} \underline{\pi}\right)$.

Индуктивный шаг (2) аналогичен шагу (4): вместо кодов $\tau$ рассматриваются числа $k \in \mathbb{N}$. Для шага (6) результат вытекает из эквивалентности

$$
\neg \exists p \in \mathbb{P}_{\mathscr{I}}^{\mathrm{M}}\left(x \in \boldsymbol{B}_{p} \wedge p \Vdash_{\mathscr{I}}^{\mathrm{M}} \Phi\right) \Longleftrightarrow \exists p \in \mathbb{P}_{\mathscr{I}}^{\mathrm{M}}\left(x \in \boldsymbol{B}_{p} \wedge p \Vdash_{\mathscr{I}}^{\mathrm{M}} \neg \Phi\right),
$$

которая доказьвается простьми стандартными рассуждениями.

Теорема 6.6. Если $x \in \operatorname{Rand}_{\mathscr{I}} \mathrm{M}$, то множество $\mathrm{M}\lceil x\rceil$ является дескриптивным континуумом.

ДокаЗАТЕльство. Результат, в принщипе, следует из леммы 4.12: равенство $\omega_{1}^{\mathbf{L}[a]}=\omega_{1}^{\mathbf{L}[a, x]}$ позволяет без труда доказать, что $\mathbf{M}\lceil x\rceil=\mathbf{L}[a, x] \cap \mathbb{N}^{\omega}$. Таким образом, здесь важно не что, а как доказывать: именно, в соответствии с нашей главной целью здесь, мы хотим дать “М-доказательство", не апеллирующее к свойствам форсинга для моделей ZFC.

Мы ограничимся проверкой Свертки для $\mathrm{M}\lceil x\rceil$ - остальные два требования проверяются при помощи похожих рассуждений. Пусть, для простоты, $n=1$, т.е. рассматривается формула $\varphi(k, l, x)$. Предположим, что $a_{1} \in \mathbb{N}^{\omega} \cap \mathrm{M}\lceil x\rceil$ и $b=\{\langle k, l\rangle$ : $\left.\varphi^{\mathrm{M}}\left(k, l, a_{1}\right)\right\} \in \mathbb{N}^{\omega}$; требуется доказать, что $b \in \mathrm{M}\lceil x\rceil$. По определению, $a_{1}=\tau_{1}{ }^{\prime} x$, где $\tau_{1} \in \mathbf{M} \cap \mathbf{B F}$. Согласно теореме 6.5, найдется "условие" $r \in \mathbb{P}_{\mathscr{I}}^{\mathrm{M}}$ такое, что $x \in \boldsymbol{B}_{r}$ и $r \Vdash_{\mathscr{I}}^{\mathrm{M}} \forall k \exists ! l \varphi\left(k, l, \tau_{1}\right)$. Положим

$$
D_{k l}=\left\{p \in \mathbb{P}_{\mathscr{I}}^{\mathrm{M}}: \boldsymbol{B}_{p} \cap \boldsymbol{B}_{r}=\varnothing \vee\left(\boldsymbol{B}_{p} \subseteq \boldsymbol{B}_{r} \wedge p \Vdash_{\mathscr{I}}^{\mathrm{M}} \forall k \exists ! l \varphi\left(k, l, \tau_{1}\right)\right)\right\} .
$$

По выбору $r$, множество $D_{k}=\bigcup_{l} D_{k l}$ плотно в $\mathbb{P}_{\mathscr{I}}^{\mathrm{M}}$ при любом $k$. Кроме того, $D_{k l}$ и $D_{k}$ все вместе определимы в М по теореме 6.4. Применяя предложение 6.3 (последнюю часть), мы находим $u \in \mathbb{N}^{\omega} \cap \mathrm{M}$ такое, что при любом $k$ множество $U_{k}=\left\{\left((u)_{k}\right)_{l}\right.$ : 
$l \in \mathbb{N}\}$ включено в $D_{k}$, а объединение $\bigcup_{c \in U_{k}} \boldsymbol{B}_{c}-\mathscr{I}$-полное множество. Разделяя эту структуру в М в соответствии с отношением к условию $r$, мы имеем $v \in \mathbb{N} \omega \cap \mathrm{M}$ такое, что каждое $V_{k}=\left\{\left((v)_{k}\right)_{l}: l \in \mathbb{N}\right\}$ удовлетворяет равенству $V_{k}=\left\{\left((v)_{k}\right)_{l}\right.$ :

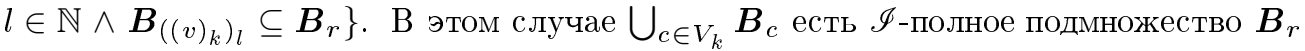
(в том смысле, что соответствующая разность принадлежит $\mathscr{I}$ ).

Положим $V_{k l}=V_{k} \cap D_{k l}$. Мы можем считать, что $\boldsymbol{B}_{c} \cap \boldsymbol{B}_{c^{\prime}}=\varnothing$ при $c \in V_{k l}$, $c^{\prime} \in V_{k l^{\prime}}, l \neq l^{\prime}$. (Конечно, вообше говоря, в этом случае $\boldsymbol{B}_{c} \cap \boldsymbol{B}_{c^{\prime}} \in \mathscr{I}$. Но мы можем выбросить все эти нежелательные пересечения - всего счетно много, так что в совокупности удаляется множество из $\mathscr{I},-$ и все опять перекодировать внутри М.) В этом предположении, найдется борелевская функция $\mathrm{F}_{\tau}: \mathbb{N}^{\omega} \rightarrow \mathbb{N} \omega$, причем $\tau \in$ $\mathbf{B F} \cap \mathbf{M}$, такая, что, для всех $k, l$ и $c \in V_{k l}$, мы имеем $\forall x^{\prime} \in \boldsymbol{B}_{c} \quad\left(\tau^{\prime} x^{\prime}(k)=l\right)$ (где $\tau^{\prime} y=$ $\left.\mathrm{F}_{\tau}(y)\right)$. В частности, это верно и для данной точки $x \in \operatorname{Rand}_{\mathscr{I}}$ М. Отсюда, обычными для форсинга рассуждениями, вьводится, что точка $y=\tau^{\prime} x \in \mathrm{M}\lceil x\rceil$ удовлетворяет $\forall k \quad \varphi^{\mathrm{M}}\left(k, y(k), a_{1}\right)$, что и требовалось.

6Г. Окончание “элементарного" доказательства теоремы 6.1. Пусть снова $a \in \mathbb{N}^{\omega}, \mathbf{M}=\mathbf{L}[a] \cap \mathbb{N}^{\omega}, \mathscr{I}$ является $\mathbf{L}[a]$-абсолютным $\sigma$-УСА-идеалом, $\mathbb{P}_{\mathscr{I}}^{\mathbf{M}}=\mathbb{P}_{\mathscr{I}}^{\mathbf{L}[a]}$ и $\operatorname{Rand}_{\mathscr{I}} \mathrm{M}=\boldsymbol{R}_{\operatorname{land}} \mathbf{L}[a]$. Следуюшая теорема аналогична теореме 4.17.

ТеОРема 6.7. Предположим, что $\varphi(x)$ - аналитическая формула с параметрами из М. Тогда найдется такой борелевский код $c \in \mathbf{B C} \cap \mathrm{M}$, что

$$
\left\{x \in \operatorname{Rand}_{\mathscr{I}} \mathrm{M}: \varphi(x) \text { истинно в } \mathrm{M}\lceil x\rceil\right\}=\operatorname{Rand}_{\mathscr{I}} \mathrm{M} \cap \boldsymbol{B}_{c} .
$$

ДокАЗАТЕЛЬство. Предположим, что $\varphi$ есть $\varphi(z, x)$, с единственньм параметром $z \in \mathbf{M}$. Пусть $c_{z} \in \mathbf{B F} \cap \mathbf{M}$ - какой-нибудь код функции-константы $F(x)=z, \forall x$; так что $c_{z}$ ' $\underline{\pi}$ всегда интерпретируется как $z$ (аналог $\breve{z}$ в изложении форсинга из п. $4 \mathrm{~A}$ ). Пусть $\varepsilon \in \mathbf{B F} \cap \mathrm{M}-$ код функции $F(x)=x, \forall x$. Тогда $\varphi(z, x)$ тождественно $\varphi\left(c_{z}^{\prime} \underline{\pi}, \varepsilon^{\prime} \underline{\pi}\right)\lceil x\rceil$ при любом $x \in \mathbb{N}^{\omega}$. Согласно теореме 6.4 , множество

$$
D=\left\{p \in \mathbb{P}_{\mathscr{I}}^{\mathrm{M}}: p \Vdash_{\mathscr{I}}^{\mathrm{M}} \varphi\left(c_{z}^{\prime} \underline{\pi}, \varepsilon^{\prime} \underline{\pi}\right) \text { или } p \Vdash_{\mathscr{I}}^{\mathrm{M}} \neg \varphi\left(c_{z}^{\prime} \underline{\pi}, \varepsilon^{\prime} \underline{\pi}\right)\right\}
$$

определимо в М, а из п. (6) определения $\Vdash_{\mathscr{I}}^{\mathrm{M}}$ следует, что оно плотно в $\mathbb{P}_{\mathscr{I}}^{\mathrm{M}}$. Отсюда, как и в доказательстве леммы 4.12 (которое применимо в данном контексте, поскольку, напомним, $\left.\mathbf{M}=\mathbf{L}[a] \cap \mathbb{N}^{\omega}\right)$, следует сушествование $p \in \mathbf{M}$ такого, что $(p)_{n} \in D$ для всех $n$ и множество $\left\{(p)_{n}: n \in \mathbb{N}\right\}$ плотно в $\mathbb{P}_{\mathscr{I}}^{\mathrm{M}}$. Последнее означает, что $\bigcup_{n} \boldsymbol{B}_{(p)_{n}}$ является $\mathscr{I}$-полньм множеством. Положим

$$
u=\left\{n:(p)_{n} \Vdash_{\mathscr{I}}^{\mathrm{M}} \varphi\left(c_{z}^{\prime} \underline{\pi}, \varepsilon^{\prime} \underline{\pi}\right)\right\} \quad \text { и } v=\mathbb{N} \backslash u=\left\{n: p_{n} \Vdash_{\mathscr{I}}^{\mathrm{M}} \neg \varphi\left(c_{z}^{\prime} \underline{\pi}, \varepsilon^{\prime} \underline{\pi}\right)\right\} .
$$

Найдутся борелевские коды $c, c^{\prime} \in \mathbf{B C} \cap$ М такие, что $\boldsymbol{B}_{c}=\bigcup_{n \in u} \boldsymbol{B}_{(p)_{n}}$ и $\boldsymbol{B}_{c^{\prime}}=\mathbb{N}^{\omega} \backslash$ $\boldsymbol{B}_{c}=\bigcup_{n \in v} \boldsymbol{B}_{(p)_{n}}$. Покажем, что $c-$ искомый код. Пусть $x \in \boldsymbol{R a n d}_{\mathscr{I}}$ М. Тогда $x \in \boldsymbol{B}_{c} \cup \boldsymbol{B}_{c^{\prime}}$. Если $x \in \boldsymbol{B}_{c}$, то $x \in \boldsymbol{B}_{(p)_{n}}$ для некоторого $n \in u$. Отсюда, согласно теореме 6.5 , следует, что формула $\varphi\left(c_{z}^{\prime} \underline{\pi}, \varepsilon^{\prime} \underline{\pi}\right)\lceil x\rceil$, т.е. $\varphi(z, x)$, истинна в $\mathrm{M}\lceil x\rceil$. Если $x \notin \boldsymbol{B}_{c}$, то $x \in \boldsymbol{B}_{c^{\prime}}$, и в силу аналогичных рассуждений формула $\varphi(z, x)$ ложна в $\mathrm{M}\lceil x\rceil$.

Теперь, чтобы завершить доказательство теоремы 6.1, остается повторить простой вьвод следствия 4.16, в котором ссылка на теорему 2.8 заменяется ссылкой на теоремы 6.6 и 6.2 . 


\section{$\S$ 7. Неразрешимость проблем}

В этом параграфе мы излагаем доказательство следуюших двух результатов.

Теорема 7.1. Ни одна из следующих импликаций не выводима в ZFC:

(I) $\neg \mathrm{PK}\left(\boldsymbol{\Pi}_{1}^{1}\right) \Longrightarrow \neg \mathrm{PK}(\mathrm{OD})$;

(II) $\neg \mathrm{BP}\left(\boldsymbol{\Delta}_{2}^{1}\right) \Longrightarrow \neg \mathrm{BP}(\mathrm{OD})$;

(III) $\neg \operatorname{LM}\left(\boldsymbol{\Delta}_{2}^{1}\right) \Longrightarrow \neg \operatorname{LM}(\mathrm{OD})$.

Теорема 7.2. Если теория ZFCI непротиворечива, то теории ZFC не противоречит предположение $\mathrm{PK}(\mathbf{R O D}) \wedge \mathrm{BP}(\mathbf{R O D}) \wedge \mathrm{LM}(\mathbf{R O D}) .{ }^{30}$

Напомним, что через OD обозначается класс всех ординально определимых множеств, а через ROD - класс всех вешественно-ординально определимых множеств (точные определения этих понятий даны ниже). Таким образом, теорема 7.1 утверждает, что из сушествования контрпримеров для свойств регулярности (причем в максимально сильной форме, так как PK вьполнено для $\Sigma_{1}^{1}$, а LM и ВР вьполнены для классов $\boldsymbol{\Sigma}_{1}^{1}$ и $\left.\boldsymbol{\Pi}_{1}^{1}\right)$ не следует сушествование әффективны $x$ контрпримеров, даже при том, очевидно, наиболее слабом понимании эффективности, которое означает $\in$-определимость с ординалами в качестве параметров.

После необходимых определений и комментариев в п. 7А мы сосредоточимся на доказательстве теоремы 7.1 а затем, на этой основе, дадим и доказательство теоремы 7.2.

7А. Определения и комментарии к теоремам. Множество $x$ можно назвать определимым, если найдется такая $\in$-формула $\varphi(v)$, без параметров и с одной свободной переменной $v$, что $x$ - единственное множество, удовлетворяющее $\varphi(x)$, т.е. $\forall v(\varphi(v) \Longleftrightarrow x=v)$. Это понятие, однако, не может быть формально определено в ZFC, другими словами, нет такой одной $\in$-формулы $\delta(x)$, которая выделяла бы сразу все определимые множества. (Мы не будем отвлекаться на объяснения, почему это так.) В то же время, более широкое понятие ординально определимого множества (т.е. определимого $\in$-формулой с ординалами в качестве параметров) допускает такое определение. Именно, рассмотрим формулу od $x$, говорящую следующее (см. [75]):

сушествуют ординал $\alpha$ и $\in$-формула $\varphi(v)$, с ординалами, меньшими $\alpha$, в роли параметров и с одной свободной переменной $v$, такие, что $x \in \mathbf{V}_{\alpha}$ и $x$ - единственное множество, удовлетворяюшее $\mathbf{V}_{\alpha} \models \varphi(x)$.

Напомним, что $\mathbf{V}_{\alpha}$ - это $\alpha$-й уровень фон Неймана (см. п. $\left.2 \mathrm{~A}\right)$, а $\models$ обозначает теоретико-модельное отношение истинности формулы в структуре.

Положим $\mathrm{OD}=\{x: \operatorname{od} x\}-$ класс всех ординально определимы $x$ множеств.

Таким образом, каждое $x \in \mathrm{OD}$ определимо $\in$-формулой вида $\mathbf{V}_{\alpha} \models \varphi(x)$, параметрами которой являются ординалы $\leqslant \alpha$. Обратно, если $x$ ординально определимо в неформальном смысле, то, используя принцип отражения (см., например, теорему 16 в [29]), мы находим ординал $\alpha$ такой, что определение релятивизуется $\mathbf{K} \mathbf{V}_{\alpha}$, и тогда $x \in \mathrm{OD}$.

\footnotetext{
${ }^{30}$ По умолчанию, в метаматематических резултатах, к которым относятся и теоремы непротиворечивости, мы предполагаем непротиворечивость ZFC, однако теорема 7.2 использует более силную гипотезу о непротиворечивости теории ZFCI, которая состоит из аксиом ZFC и аксиомы "существует строго недостижимьй кардинал".
} 
Аналогично определяется класс $\mathbf{R O D}=\{x: \operatorname{rod} x\}$ всех вещественно-ординально определимы $x^{31}$ множеств, т.е. тех, которые определимы формулами, содержашими ординалы и элементы $\mathbb{N}^{\omega}$ в качестве параметров. Здесь $\operatorname{rod} x$ представляет собой модификацию формулы od $x$, состоящую в том, что $\varphi$, кроме ординалов, может содержать элементы $\mathbb{N}^{\omega} \cap \mathbf{V}_{\alpha}$ в качестве параметров. (См. [75] или [29; разд. 14] о деталях, связанных с ординальной определимостью.)

Понятно, что Ord $\subseteq$ OD, более того, $\mathbf{L} \subseteq \mathrm{OD}$, ибо для любого $x \in \mathbf{L}$ имеется ординал $\xi$ такой, что $x=\bar{F}_{\xi}$. Далее, $\Sigma_{\infty}^{1} \subseteq$ OD, а класс $\boldsymbol{\Sigma}_{\infty}^{1}$ всех проективных множеств пространства $\mathbb{N}^{\omega}$ включен в ROD, так что теорема 7.2 влечет

СлЕДСТВИЕ 7.3. Предположим, что теория ZFCI непротиворечива. Тогда

(i) предложсение о том, что все проективные множества имеют свойство совершенного ядра, свойство Бэра и $\lambda$-измеримы, не противоречит ZFC, а потому, вследствие теоремь 3.11 , это предложение неразрешимо в $\mathrm{ZFC}$

(ii) следовательно, в силу теоремь 3.11, предложения $\operatorname{PK}\left(\boldsymbol{\Pi}_{1}^{1}\right), \operatorname{LM}\left(\boldsymbol{\Delta}_{2}^{1}\right)$, $\operatorname{BP}\left(\boldsymbol{\Delta}_{2}^{1}\right), \operatorname{LM}\left(\boldsymbol{\Sigma}_{2}^{1}\right), \operatorname{BP}\left(\boldsymbol{\Sigma}_{2}^{1}\right)$ (см. диаграмму из введения) такжее непротиворечивы и неразрешимы в ZFC.

Теорема 7.1 связана с такой формой проблемы свойств регулярности точечных множеств: можно ли назвать контрпримеры ${ }^{32}$ к свойствами регулярности, при условии, что такие контрпримеры существуют. Теорема дает отрицательный ответ.

Самому результату можно придать форму, в которой ординалы отсутствуют, т.е. речь идет о "чистой” определимости, однако, по указанным вьше причинам, следствие вьглядит более "метаматематическим", чем сама теорема.

СлЕДСТВИЕ 7.4. Нет такой $\in$ формулы с одной свободной переменной, которая удовледворяет хотя бь одному из трех следующих условий:

(i) она, доказуемо в $\mathbf{Z F C}+\neg \mathrm{PK}\left(\boldsymbol{\Pi}_{1}^{1}\right)$, определяет подмножсество $\mathbb{N}^{\omega}$, не имеюшее свойства совершенного ядра;

(ii) она, доказуемо в $\mathbf{Z F C}+\neg \operatorname{BP}\left(\boldsymbol{\Delta}_{2}^{1}\right)$, определяет подмножсество $\mathbb{N} \omega$, не имеющее свойства Бэра;

(iii) она, доказуемо в $\mathbf{Z F C}+\neg \operatorname{LM}\left(\boldsymbol{\Delta}_{2}^{1}\right)$, определяет $\boldsymbol{\lambda}$-неизмеримое подмножество $\mathbb{N}^{\omega}$.

Доказательства теорем 7.1 и 7.2, следующие ниже в этом параграфе, включают анализ трех различных моделей $\mathbf{Z F C}$, которые получаются расширением класса $\mathbf{L}$ посредством:

1) одной генерической склеивающей функции $\omega \stackrel{\text { на }}{\longrightarrow} \omega_{1}^{\mathrm{L}}-$ этим будет доказана теорема 7.1 в частях (II) и (III);

\footnotetext{
${ }^{31}$ Перевод термина "real-ordinal definable", где первое слово отражкает возможность брать точки $\mathbb{N}^{\omega}$ в качестве параметров определения, дополнителшно к ординалам. Употребление слова "real" в роли имени существительного применительно к точкам $\mathbb{N}^{\omega}$ достаточно характерно для англоязычной литературы по дескриптивной теории множеств; оно базируется на том отождествлении точек $\mathbb{N}^{\omega}$ с иррациональными вещественньми числами, о котором шла речь в сноске 12. K сожалению, приемлемого перевода слова "real" в этом его значении на русский язьк, совместимого с традициями русского математического язька, по-видимому, нет.

${ }^{32} \mathrm{O}$ дискуссиях в ранней дескриптивной теории множеств в связи с "называемьпм" примерами, в противовес "чистьм" доказательствам существования, см. литературу, упомянутую в сноске 9 .
} 
2) совокупности из генерических склеивающих функций $\omega \stackrel{\text { на }}{\longrightarrow} \omega_{1}^{\mathrm{L}}$ в числе $\aleph_{2}^{\mathrm{L}}-$ этим будет доказана теорема 7.1 в части (I);

3) склейки до строго недостижимого кардинала - так доказьвается теорема 7.2. Для всех трех случаев выкладки будут, впрочем, иметь много общих моментов.

7Б. Первая модель - одна склеивающая функция. Для доказательства теоремы 7.1 в частях (II) и (III), возьмем класс $\mathbf{L}$ всех конструктивных множеств в качестве исходной модели и форсинг $\mathbb{C}\left(\omega_{1}^{\mathbf{L}}\right)=\operatorname{Coll}\left(\mathbb{N}, \omega_{1}^{\mathbf{L}}\right)$, предназначенный для "склейки" $\omega_{1}^{\mathbf{L}}$. Таким образом, $\mathbb{C}\left(\omega_{1}^{\mathbf{L}}\right)$ состоит из всех функций $p$ таких, что $\operatorname{dom} p \in \mathbb{N}$ и $\operatorname{ran} p \subseteq \omega_{1}^{\mathrm{L}}$. Упорядочим $\mathbb{C}\left(\omega_{1}^{\mathrm{L}}\right)$ обратно включению. Тогда любое $\mathbb{C}\left(\omega_{1}^{\mathrm{L}}\right)$-генерическое множество $G \subseteq \mathbb{C}\left(\omega_{1}^{\mathbf{L}}\right)$ производит генерическую склейку $f[G]=\bigcup G: \mathbb{N} \stackrel{\text { на }}{\longrightarrow} \omega_{1}^{\mathbf{L}}$, и в то же время $G=\{f[G]\lceil n: n \in \mathbb{N}\}$. Согласно теореме 4.4, следуюшая теорема влечет теорему 7.1 (II), (III).

Tеорема 7.5. Пусть $G \subseteq \mathbb{C}\left(\omega_{1}^{\mathbf{L}}\right)$ является $\mathbb{C}\left(\omega_{1}^{\mathrm{L}}\right)$-генерическим над $\mathbf{L}$ множеством. Тогда в $\mathbf{L}[G]$ выполнены $\mathrm{BP}(\mathrm{OD})$ и $\mathrm{LM}(\mathrm{OD})$, но не имеет места ни $\operatorname{LM}\left(\boldsymbol{\Delta}_{2}^{1}\right)$, ни $\operatorname{BP}\left(\boldsymbol{\Delta}_{2}^{1}\right)$.

В ходе доказательства этой теоремы, т.е. до конца п. 7В, мы фиксируем $\mathbb{C}\left(\omega_{1}^{\mathbf{L}}\right)$-генерическое над $\mathbf{L}$ множество $G$. "Отрицательная" часть теоремы не представляет затруднений. В силу того, что функция $f[G] \in \mathbf{L}[G]$ отображает $\omega$ на $\omega_{1}^{\mathrm{L}}$, мы имеем $\omega_{1}^{\mathbf{L}}<\omega_{1}^{\mathbf{L}[G]}$, следовательно, найдется $w \in \mathbf{L}[G] \cap \mathbf{W O}_{\omega_{1}^{\mathbf{L}}}$. Это позволяет получить точку $a \in \mathbb{N}^{\omega}$ такую, что $\mathbf{L}[a]=\mathbf{L}[f[G]]=\mathbf{L}[G]$. Нарушение $\operatorname{BP}\left(\boldsymbol{\Delta}_{2}^{1}\right)$ и $\operatorname{LM}\left(\boldsymbol{\Delta}_{2}^{1}\right)$ в $\mathbf{L}[G]$ следует теперь из теоремы 3.11 .

"Положительная" же часть требует значительно больше работы. Согласно лемме 3.8 , достаточно доказать, что в $\mathbf{L}[G]$ любое OD-множество $\mathscr{I}$-измеримо, каков бы ни был $\mathbf{L}$-абсолютньй $\sigma$-УСА-идеал $\mathscr{I}$. Доказательство базируется на следуюшей лемме, в которой $\Lambda$ ("пустая" функция) - самьй слабый элемент $\mathbb{C}\left(\omega_{1}^{\mathbf{L}}\right)$, а $\breve{z}_{i}-$ канонические $\mathbb{C}\left(\omega_{1}^{\mathbf{L}}\right)$-имена множеств $z_{i}$ (ясно, что $\breve{z}_{i}[G]=z_{i}$ для $\left.i=1, \ldots, n\right)$.

Лемма 7.6. Предположим, что $x \in \mathbf{L}[G] \cap \mathbb{N}^{\omega}, \omega_{1}^{\mathbf{L}[x]}=\omega_{1}^{\mathbf{L}}, \varphi\left(v_{1}, \ldots, v_{n}\right)$ есть $\in$-формула, а $z_{1}, \ldots, z_{n} \in \mathbf{L}[x]-$ произвольние множсества. Тогда

$$
\varphi\left(z_{1}, \ldots, z_{n}\right) \text { истинно в } \mathbf{L}[G] \Longleftrightarrow \Lambda \Vdash_{\mathbb{C}\left(\omega_{1}^{\mathbf{L}}\right)}^{\mathbf{L}[x]} \varphi\left(\breve{z}_{1}, \ldots, \breve{z}_{n}\right) \text {. }
$$

Следовательно, если $X \in \mathbf{L}[G], X \subseteq \mathbf{L}, X \in \mathrm{OD}$ в $\mathbf{L}$, mо $X \in \mathbf{L}$.

Приняв эту лемму, рассмотрим, рассуждая в $\mathbf{L}[G]$, произвольный $\mathbf{L}$-абсолютньй $\sigma$-УСА-идеал $\mathscr{I}$. Заметим, что множество $\mathbf{L} \cap \mathbb{N}^{\omega}$ счетно в $\mathbf{L}[G]$ (поскольку $\omega_{1}^{\mathbf{L}}<$

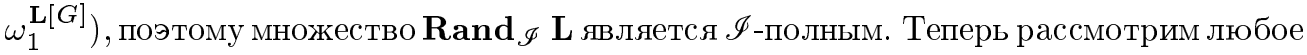
OD-множество $X \subseteq \mathbb{N}^{\omega}$. Таким образом, $X=\left\{x \in \mathbb{N}^{\omega}: \varphi\left(x, \alpha_{1}, \ldots, \alpha_{n}\right)\right\}$, где $\alpha_{1}, \ldots, \alpha_{n}$ - ординалы. Обозначим через $\psi(x)$ формулу:

$$
\text { “в } \mathbf{L}[x] \text { истинно } \Lambda \Vdash_{\mathbb{C}\left(\omega_{1}^{\mathbf{L}}\right)}^{\mathbf{L}[x]} \varphi\left(\breve{x}, \breve{\alpha}_{1}, \ldots, \breve{\alpha}_{n}\right) \text { ”. }
$$

По лемме 7.6, для любого $x \in \mathbf{R a n d}_{\mathscr{I}} \mathbf{L}$, в $\mathbf{L}[G]$ вьполнена следующая эквивалентность: $x \in X \Longleftrightarrow \psi(x)$. (Заметим, что $\omega_{1}^{\mathbf{L}[x]}=\omega_{2}^{\mathbf{L}}$ следует из леммы 4.12.) Теперь $\mathscr{I}$-измеримость $X$ следует из теоремы 4.17 . 
ЗАмечАнИЕ 7.7. Данное доказательство $\mathrm{BP}(\mathrm{OD})$ и $\mathrm{LM}(\mathrm{OD})$ в $\mathbf{L}[G]$ легко преврашается в доказательство $\operatorname{BP}(\mathrm{OD}(a))$ и $\operatorname{LM}(\mathrm{OD}(a))$ для любого $a \in \mathbf{L}[G] \cap \mathbb{N}^{\omega}$ такого, что $\omega_{1}^{\mathbf{L}[a]}=\omega_{1}^{\mathbf{L}}$, где $\mathrm{OD}(a)$ означает, что, кроме ординалов, $a$ также можно использовать в качестве параметра определимости.

7В. Доказательство ключевой леммы. Доказательство леммы 7.6 опирается на следующую лемму.

Лемма 7.8. Если $x \in \mathbf{L}[G] \cap \mathbb{N}^{\omega}$ удовлетворяет $\omega_{1}^{\mathbf{L}[x]}=\omega_{1}^{\mathbf{L}}$, то класс $\mathbf{L}[G]$ является $\mathbb{C}\left(\omega_{1}^{\mathbf{L}}\right)$-генерическим расширением $\mathbf{L}[x]$. (Напомним: $\mathbb{C}\left(\omega_{1}^{\mathbf{L}}\right)=\operatorname{Coll}\left(\mathbb{N}, \omega_{1}^{\mathbf{L}}\right)$.)

ДокАЗАТЕльство. Зафиксируем $\mathbb{C}\left(\omega_{1}^{\mathbf{L}}\right)$-имя $t \in \mathbf{L}$ такое, что $x=t[G]$. Индукцией по $\xi<\operatorname{Ord}$ мы определяем множества $A_{\xi} \subseteq \mathbb{C}\left(\omega_{1}^{\mathbf{L}}\right)$. Положим

$$
\begin{aligned}
A_{0}= & \left\{p \in \mathbb{C}\left(\omega_{1}^{\mathbf{L}}\right): \forall k \forall \gamma<\omega_{1}^{\mathbf{L}}\left(\left(p \Vdash_{\mathbb{C}\left(\omega_{1}^{\mathbf{L}}\right)}^{\mathbf{L}} t(\breve{k})=\breve{\gamma}\right) \Longrightarrow x(k)=\gamma\right)\right\}, \\
A_{\xi+1}= & \left\{p \in A_{\xi}: \text { для каждого плотного в } \mathbb{C}\left(\omega_{1}^{\mathbf{L}}\right) \text { множества } D \in \mathbf{L},\right. \\
\left.D \subseteq \mathbb{C}\left(\omega_{1}^{\mathbf{L}}\right), \text { найдется } q \in A_{\xi} \cap D \text { такое, что } q \leqslant p\right\}, & \\
A_{\vartheta}= & \bigcap_{\xi<\vartheta} A_{\xi} \text { для предельных ординалов } \vartheta
\end{aligned}
$$

(напомним, что $q \leqslant p$ означает $p \subseteq q$ в $\left.\mathbb{C}\left(\omega_{1}^{\mathbf{L}}\right)=\operatorname{Coll}\left(\mathbb{N}, \omega_{1}^{\mathbf{L}}\right)\right)$. Раз речь идет об убываюших подмножествах $\mathbb{C}\left(\omega_{1}^{\mathrm{L}}\right)$, найдется ординал $\zeta$ такой, что $A_{\zeta}=A_{\zeta+1}=A_{\eta}$ для всех $\eta>\zeta$. Можно показать, что это “предельное" множество $\Sigma=A_{\zeta}$ состоит из всех тех условий $p$, которые не вынуждают для $t$ ничего несовместимого с имеюшимися свойствами $x=t[G]$, но мы не будем углубляться в эту тему.

Имеют место следуюшие утверждения о множестве $\Sigma$.

(1) $\Sigma \in \mathbf{L}[x]$. (В самом деле, $\mathbb{C}\left(\omega_{1}^{\mathbf{L}}\right) \in \mathbf{L}$, а отношение $\Vdash_{\mathbb{C}\left(\omega_{1}^{\mathbf{L}}\right)}^{\mathbf{L}}$ определимо в $\mathbf{L}$, поэтому $A_{0} \in \mathbf{L}[x]$ и последовательность множеств $A_{\xi}$ принадлежит $\mathbf{L}[x]$.)

(2) Если множество $D \in \mathbf{L}, D \subseteq \mathbb{C}\left(\omega_{1}^{\mathbf{L}}\right)$ плотно в $\mathbb{C}\left(\omega_{1}^{\mathbf{L}}\right)$, то $D \cap \Sigma$, плотно в $\Sigma$. (Это вытекает из того, что $\Sigma=A_{\zeta}=A_{\zeta+1}$.) Сразу отметим следствие: $\Sigma$ не имеет $\subseteq$-максимальных элементов.

(3) $G \subseteq \Sigma$. $\left(G \subseteq A_{\xi}\right.$ вьводится индукцией по $\xi$ из генеричности $G$.)

(4) Если $G^{\prime} \subseteq \bar{\Sigma}$ является $\mathbb{C}\left(\omega_{1}^{\mathrm{L}}\right)$-генерическим над $\mathbf{L}$, то $t\left[G^{\prime}\right]=t[G]=x$. (Вообще, легко видеть, что это верно даже при $G^{\prime} \subseteq A_{0}$.)

(5) $G$ является $\Sigma$-генерическим над $\mathbf{L}[x]$ множеством.

Для доказательства (5) предположим противное. Согласно теореме 4.3, существует "условие" $p \in G$ такое, что любое $\mathbb{C}\left(\omega_{1}^{\mathbf{L}}\right)$-генерическое над $\mathbf{L}$ множество $G^{\prime} \subseteq$ $\mathbb{C}\left(\omega_{1}^{\mathbf{L}}\right)$, содержащее $p$, не будет $\Sigma$-генерическим над $\mathbf{L}[x]$. (Замечание: из (4) следует, что $t\left[G^{\prime}\right]=x$, а потому множество $\Sigma$, построенное в $\mathbf{L}\left[G^{\prime}\right]$, совпадает с множеством $\Sigma$, построенным в $\mathbf{L}[G]$.) Теперь рассмотрим произвольное $\Sigma$-генерическое над $\mathbf{L}[x]$ множество $G^{\prime} \subseteq \mathbb{C}\left(\omega_{1}^{\mathbf{L}}\right)$, содержашее $p$ (теорема 4.4 ). Из (2) следует, что $G^{\prime}$ также является $\mathbb{C}\left(\omega_{1}^{\mathbf{L}}\right)$-генерическим над $\mathbf{L}$, противоречие.

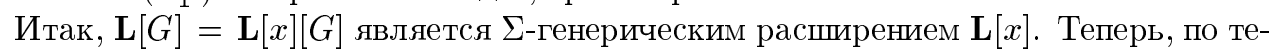
ореме 4.2(ii), для доказательства леммы 7.8 вполне достаточно установить, что множество $\Sigma$ содержит плотное подмножество $\Sigma^{\prime} \in \mathbf{L}[x]$, которое порядково изоморфно $\mathbb{C}\left(\omega_{1}^{\mathbf{L}}\right)$ в $\mathbf{L}[x]$. Напомним, что предполагается $\omega_{1}^{\mathbf{L}[x]}=\omega_{1}^{\mathbf{L}}$, так что $\omega_{1}^{\mathbf{L}}$ все еще является первым несчетным ординалом в $\mathbf{L}[x]$. Следовательно, $\mathbb{C}\left(\omega_{1}^{\mathbf{L}}\right)$ является деревом 
высоты $\omega$ с $\omega_{1}$-ветвлениями в $\mathbf{L}[x]$. Отсюда вытекает, что для построения искомого $\Sigma^{\prime}$ достаточно проверить следуюшее: каково бы ни было “условие" $p \in \Sigma$, множество $\Sigma_{p}=\{q \in \Sigma: q \leqslant p\}$ несчетно в $\mathbf{L}[x]$.

Предположим противное: $\Sigma_{p}$ счетно в $\mathbf{L}[x]$. Тогда, взяв любое $\Sigma$-генерическое над $\mathbf{L}[x]$ множество $G^{\prime} \subseteq \Sigma$, содержашее $p$, мы получим $\omega_{1}^{\mathbf{L}\left[x, G^{\prime}\right]}=\omega_{1}^{\mathbf{L}[x]}=\omega_{1}^{\mathbf{L}}$. Однако, как мы видели вьше, в этом случае $G^{\prime}$ будет также и $\mathbb{C}\left(\omega_{1}^{\mathbf{L}}\right)$-генерическим над $\mathbf{L}$, что влечет счетность $\omega_{1}^{\mathrm{L}}$ в $\mathbf{L}\left[G^{\prime}\right]$, противоречие.

ДоКАЗАТЕЛЬСТВо ЛЕммы 7.6. Предположим противное. Тогда по теореме 4.3 и лемме 7.8 , существуют “условия” $p, q \in \mathbb{C}\left(\omega_{1}^{\mathbf{L}}\right)$ такие, что $p \Vdash_{\mathbb{C}\left(\omega_{1}^{\mathbf{L}}\right)}^{\mathbf{L}[x]} \varphi\left(\breve{z}_{1}, \ldots, \breve{z}_{n}\right)$ и в то же самое время $q \Vdash_{\mathbb{C}\left(\omega_{1}^{\mathbf{L}}\right)}^{\mathbf{L}[x]} \neg \varphi\left(\breve{z}_{1}, \ldots, \breve{z}_{n}\right)$. Можно считать, что $\operatorname{dom} p=\operatorname{dom} q=$ $m \in \mathbb{N}$. Через $\mathbb{C}\left(\omega_{1}^{\mathbf{L}}\right)_{\leqslant p}$ обозначим множество всех $p^{\prime} \in \mathbb{C}\left(\omega_{1}^{\mathbf{L}}\right), p^{\prime} \leqslant p$. Аналогично определим $\mathbb{C}\left(\omega_{1}^{\mathbf{L}}\right)_{\leqslant q}$. Теперь если $p^{\prime} \in \mathbb{C}\left(\omega_{1}^{\mathbf{L}}\right)_{\leqslant p}$, то определим $h\left(p^{\prime}\right) \in \mathbb{C}\left(\omega_{1}^{\mathbf{L}}\right)_{\leqslant q}$ так, что $\operatorname{dom} h\left(p^{\prime}\right)=\operatorname{dom} p^{\prime}, h\left(p^{\prime}\right)(k)=q(k)$ при $k \in \operatorname{dom} p$ и $h\left(p^{\prime}\right)(k)=p^{\prime}(k)$ при $k \in$ $\operatorname{dom} p^{\prime} \backslash \operatorname{dom} p$. Понятно, что $h \in \mathbf{L}-$ порядковьй изоморфизм $\mathbb{C}\left(\omega_{1}^{\mathbf{L}}\right)_{\leqslant p}$ на $\mathbb{C}\left(\omega_{1}^{\mathbf{L}}\right)_{\leqslant q}$.

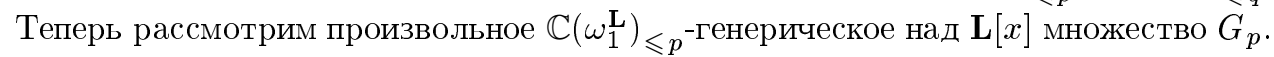
Тогда $p \in G_{p}$ и $G_{q}=\left\{h\left(p^{\prime}\right): p^{\prime} \in G_{p}\right\} \subseteq \mathbb{C}\left(\omega_{1}^{\mathbf{L}}\right)_{\leqslant q}-\mathbb{C}\left(\omega_{1}^{\mathbf{L}}\right)_{\leqslant q}$-генерическое, над $\mathbf{L}[x]$, множество, содержашее $q$. Легко видеть, что множество $G_{p}^{\prime}=G_{p} \cup\left\{p^{\prime} \in \mathbb{C}\left(\omega_{1}^{\mathbf{L}}\right)\right.$ : $\left.p^{\prime} \geqslant p\right\}$ является уже $\mathbb{C}\left(\omega_{1}^{\mathbf{L}}\right)$-генерическим над $\mathbf{L}[x]$, откуда, по выбору $p$, следует, что формула $\varphi\left(z_{1}, \ldots, z_{n}\right)$ истинна в $\mathbf{L}[x]\left[G_{p}\right]$. По тем же соображениям эта формула ложна в $\mathbf{L}[x]\left[G_{q}\right]$. Но $\mathbf{L}[x]\left[G_{p}\right]=\mathbf{L}[x]\left[G_{q}\right]$ (поскольку $h \in \mathbf{L}$ ), противоречие.

Для доказательства последнего утверждения предположим, что, в $\mathbf{L}[G]$, вьполнено $X=\left\{x \in \mathbf{L}: \varphi\left(x, \alpha_{1}, \ldots, \alpha_{n}\right)\right\}$, где $\alpha_{1}, \ldots, \alpha_{n}$ - ординалы. Тогда

$$
x \in X \Longleftrightarrow \Lambda \Vdash_{\mathbb{C}\left(\omega_{1}^{\mathbf{L}}\right)}^{\mathbf{L}} \varphi\left(\breve{x}, \breve{\alpha}_{1}, \ldots, \breve{\alpha}_{n}\right)
$$

по уже доказанному. Однако $\Vdash_{\mathbb{C}\left(\omega_{1}^{\mathbf{L}}\right)}^{\mathbf{L}}$ выразимо в $\mathbf{L}$ по теореме 4.3

$\square$ (теорема 7.5 и теорема 7.1 - части (II), (III))

7Г. Вторая модель - склеивающие функции в числе $\aleph_{2}^{L}$. Для доказательства теоремы 7.1 в ее части (I) снова возьмем класс $\mathbf{L}$ в качестве исходной модели и произведение $\aleph_{2}$ экземпляров форсинга $\mathbb{C}\left(\omega_{1}^{\mathrm{L}}\right)=\operatorname{Coll}\left(\mathbb{N}, \omega_{1}^{\mathrm{L}}\right)$ с конечной базой, которое мы обозначим через $\mathbf{P}$. Другими словами, $\mathbf{P}$ состоит из всех функций $p$ таких, что $\operatorname{dom} p \subseteq \omega_{2}^{\mathbf{L}}$ конечно и $p(\xi) \in \mathbb{C}\left(\omega_{1}^{\mathbf{L}}\right)$ для всех $\xi \in \operatorname{dom} p$. Порядок на $\mathbf{P}$ определяется естественным образом: $p \leqslant q$ (т.е. $p$ сильнее, чем $q)$, когда $\operatorname{dom} q \subseteq \operatorname{dom} p$ и $p(\xi) \leqslant q(\xi)$ в $\mathbb{C}\left(\omega_{1}^{\mathbf{L}}\right)$ для всех $\xi \in \operatorname{dom} q$. Таким образом, пустая функция $\Lambda$ - это наибольший (и самый слабый) элемент $\mathbf{P}$. Положим

$$
\mathbf{P}_{<\vartheta}=\{p \in \mathbf{P}: \operatorname{dom} p \subseteq \vartheta\}, \quad \mathbf{P}_{\geqslant \vartheta}=\left\{p \in \mathbf{P}: \operatorname{dom} p \subseteq \omega_{2}^{\mathrm{L}} \backslash \vartheta\right\},
$$

а также $\mathbf{P}_{\leqslant \vartheta}=\mathbf{P}_{<\vartheta+1}$ и $\mathbf{P}_{>\vartheta}=\mathbf{P}_{\geqslant \vartheta+1}$, для каждого $\vartheta<\omega_{2}^{\mathrm{L}}$.

Следуюшая теорема достаточна для доказательства теоремы 7.1(I).

Теорема 7.9. Пусть $G \subseteq \mathbf{P}$ является $\mathbf{P}$-генерическим над $\mathbf{L}$ множеством. Тогда в $\mathrm{L}[G]$ имеет место $\mathrm{PK}(\mathrm{OD})$, но не вилолняется $\mathrm{PK}\left(\boldsymbol{\Pi}_{1}^{1}\right)$.

В ходе доказательства этой теоремы, т.е. до конца п. 7Д, мы фиксируем Р-генерическое над $\mathbf{L}$ множество $G \subseteq \mathbf{P}$. Оно производит набор $\mathbb{C}\left(\omega_{1}^{\mathbf{L}}\right)$-генерических множеств 
$G_{\xi}=\{p(\xi): p \in G\}, \xi<\omega_{2}^{\mathbf{L}}$, и набор "склеек" $f_{\xi}[G]=\bigcup G_{\xi}: \mathbb{N} \stackrel{\text { на }}{\longrightarrow} \omega_{1}^{\mathbf{L}}$. Положим $G_{<\vartheta}=G \cap \mathbf{P}_{<\vartheta}$ и аналогично $G_{\leqslant \vartheta}, G_{>\vartheta}, G_{\geqslant \vartheta}$.

"Отрицательная" часть теоремы не представляет больших трудностей: она опирается на следуюший простой факт.

Лемма 7.10. Любая антищепь $A \subseteq \mathbf{P}, A \in \mathbf{L}$ имеет мощность $\leqslant \omega_{1}^{\mathrm{L}}$ в $\mathbf{L}$.

ДокАЗАтельство. Рассуждаем в $\mathbf{L}$. Можно считать, что $A$ - максимальная антицепь. Положим $\xi_{0}=0$. Если $\xi_{n}<\omega_{2}^{\mathbf{L}}$ определено, то множество $\mathbf{P}_{<\xi_{n}}$ имеет, очевидно, мошность $\leqslant \omega_{1}^{\mathrm{L}}$, апотому, вследствие максимальности $A$, найдется ординал $\xi, \xi_{n}<\xi<\omega_{2}^{\mathbf{L}}$, такой, что для всякого $p \in \mathbf{P}_{<\xi_{n}}$ существует "условие" $q \in A \cap \mathbf{P}_{<\xi}$, совместимое с $p$ в $\mathbf{P}$. Наименьший из таких ординалов $\xi$ обозначим через $\xi_{n+1}$. Тогда $\vartheta=\sup _{n} \xi_{n}<\omega_{2}^{\mathbf{L}}$. Остается показать, что $A \subseteq \mathbf{P}_{<\vartheta}$. Пусть, напротив, $r \in A \backslash \mathbf{P}_{<\vartheta}$. Тогда $p=r\left\lceil(\vartheta \cap \operatorname{dom} p)\right.$ принадлежит $\mathbf{P}_{<\vartheta}$, так что $p \in \mathbf{P}_{<\xi_{n}}$ для некоторого $n$, и тогда по построению найдется "условие" $q \in A \cap \mathbf{P}_{<\vartheta}$, совместимое с $p$. Тогда $q$ совместимо и с $r$, поскольку по построению $\operatorname{dom} q \cap \operatorname{dom} r=\operatorname{dom} p \cap \operatorname{dom} r$. Однако $q$, $r$ принадлежат $A$ и заведомо $q \neq r$, так как $r \notin \mathbf{P}_{<\vartheta},-$ противоречие.

Из леммы немедленно следует $\omega_{1}^{\mathbf{L}[G]} \leqslant \omega_{2}^{\mathbf{L}}$ по теореме 4.2(i). С другой стороны, функция $f_{0}[G] \in \mathbf{L}[G]$ (вообще, любая из функций $f_{\xi}[G]$ ) отображает $\omega$ на $\omega_{1}^{\mathbf{L}}$, так что $\omega_{1}^{\mathbf{L}}<\omega_{1}^{\mathbf{L}[G]}$. Отсюда следует $\omega_{1}^{\mathbf{L}[G]}=\omega_{2}^{\mathbf{L}}$, более того, найдется $a \in \mathbf{L}[G] \cap \mathbf{W} \mathbf{O}_{\omega_{1}^{\mathbf{L}}}$. Такое $a$ удовлетворяет $\omega_{1}^{\mathbf{L}[a]}=\omega_{1}^{\mathbf{L}[G]}=\omega_{2}^{\mathbf{L}}$, так что $\mathbf{L}[a] \cap \mathbb{N}^{\omega}$ счетно в $\mathbf{L}[G]$, и для вьвода $\neg \mathrm{PK}\left(\boldsymbol{\Pi}_{1}^{1}\right)$ в $\mathbf{L}[G]$ остается применить теорему 3.11 .

"Положительная" же часть теоремы 7.9, т.е. проверка $\mathrm{PK}(\mathrm{OD})$ в $\mathbf{L}[G]$ (см. п. 7 Д), более громоздка и опирается на ряд лемм.

ЛЕмма 7.11. Если $x \in \mathbf{L}[G] \cap \mathbb{N}^{\omega}$, то $x \in \mathbf{L}\left[G_{<\vartheta}\right]$ для некоторого $\vartheta<\omega_{2}^{\mathbf{L}}$.

ДокаЗАтельство. По теореме 4.1, найдется $\mathbf{P}$-имя $t \in \mathbf{L}$ такое, что $x=t[G]$. Рассуждая в $\mathbf{L}$, положим $D_{n k}=\left\{p \in \mathbf{P}: p \Vdash_{\mathbf{L}}^{\mathbf{P}} t(\breve{n})=\breve{k}\right\}$ и $D_{n}=\bigcup_{k \in \mathbb{N}} D_{n k}$, выберем максимальную антицеп $A_{n} \subseteq D_{n}$ в каждом $D_{n}$ и положим $A=\bigcup_{n} A_{n}$. Из леммы 7.10 следует $\operatorname{card} A<\Omega$, поэтому найдется ординал $\vartheta<\omega_{2}^{\mathrm{L}}$ такой, что $A \subseteq \mathbf{P}_{<\vartheta}$. Теперь нетрудно проверить, что

$$
x(n)=k \Longleftrightarrow G \cap D_{n k} \neq \varnothing \Longleftrightarrow G \cap D_{n k} \cap A \neq \varnothing \Longleftrightarrow G_{<\vartheta} \cap D_{n k} \neq \varnothing,
$$

откуда и следует $x \in \mathbf{L}\left[G_{<\vartheta}\right]$.

ЛЕмма 7.12 (ср. с леммой 7.8). Если $x \in \mathbf{L}[G] \cap \mathbb{N}^{\omega}$, то класс $\mathbf{L}[G]$ является $\mathbf{P}$-генерическим расширением $\mathbf{L}[x]$.

ДокАЗАТЕЛЬСтво. Используя лемму 7.11, находим ординал $\vartheta<\omega_{2}^{\mathrm{L}}$ такой, что $x \in \mathbf{L}\left[G_{<\vartheta}\right]$. Можно предполагать, что $\vartheta \geqslant \omega_{1}^{\mathbf{L}}$. Будем считать, для простоты, что $\vartheta=\omega_{1}^{\mathbf{L}}$; обший случай сводится к этому при помоши любой биекции $b: \omega_{1}^{\mathbf{L}} \stackrel{\text { на }}{\longrightarrow} \vartheta$ в $\mathbf{L}$. В этом предположении, рассмотрим множество $\mathbf{P}^{\prime}$ всех "условий” $p \in \mathbf{P}_{<\omega_{1}^{L}}$ таких, что либо $p=\Lambda$, либо $\operatorname{dom} p=\{0\} \cup\{p(0)(k): k \in \operatorname{dom} p(0)\}$ и $\operatorname{dom} p(\xi)=\operatorname{dom} p(0)$ для каждого $\xi \in \operatorname{dom} p$.

Нетрудно проверить, что $\mathbf{P}^{\prime} \in \mathbf{L}, \mathbf{P}^{\prime}$ плотно в $\mathbf{P}_{<\omega_{1}^{\mathbf{L}}}$ и $\mathbf{P}^{\prime}$ порядково изоморфно множеству $\mathbb{C}\left(\omega_{1}^{\mathbf{L}}\right)=\operatorname{Coll}\left(\mathbb{N}, \omega_{1}^{\mathbf{L}}\right)$ в $\mathbf{L}$ (каждое из этих двух множеств - дерево высоты $\omega$ 
с $\omega_{1}^{\mathrm{L}}$-ветвлениями в смысле порядка, совпадающего с $\subseteq$, т.е. обратного $\left.\leqslant\right)$. Отсюда следует, что $\mathbf{L}\left[G_{<\vartheta}\right]=\mathbf{L}[F]$, где множество $F \subseteq \mathbb{C}\left(\omega_{1}^{\mathbf{L}}\right)$ является $\mathbb{C}\left(\omega_{1}^{\mathbf{L}}\right)$-генерическим над $\mathbf{L}$. Совершенно аналогично доказательству леммы 7.8 , найдется дерево $\Sigma \subseteq \mathbb{C}\left(\omega_{1}^{\mathbf{L}}\right), \Sigma \in \mathbf{L}[x]$, без $\subseteq$-максимальных элементов, такое, что $F \subseteq \Sigma$ и $F$ является $\Sigma$-генерическим над $\mathbf{L}[x]$.

Теперь мы рассмотрим класс $\mathbf{L}\left[G_{\leqslant} \mathbf{L}\right]$. Очевидно, что $\mathbf{P}_{\leqslant} \omega_{1}^{\mathbf{L}}$ порядково изоморффно произведению $\mathbf{P}_{<\omega_{1}^{\mathbf{L}}} \times \mathbb{C}\left(\omega_{1}^{\mathbf{L}}\right)$, так что по теореме $4.2(\mathrm{iii})$ класс $\mathbf{L}\left[G_{\leqslant} \omega_{1}^{\mathbf{L}}\right]$ является $\mathbb{C}\left(\omega_{1}^{\mathbf{L}}\right)$-генерическим расширением класса $\mathbf{L}\left[G_{<\omega_{1}^{\mathbf{L}}}\right]=\mathbf{L}[F]$, а тогда, по теореме 4.2(iv), и $\Sigma \times \mathbb{C}\left(\omega_{1}^{\mathbf{L}}\right)$-генерическим расширением $\mathbf{L}[x]$.

Однако, вследствие указанных вьше свойств $\Sigma$, множество $\Sigma \times \mathbb{C}\left(\omega_{1}^{\mathbf{L}}\right)$ порядково изоморфно самому $\mathbb{C}\left(\omega_{1}^{\mathbf{L}}\right)$. Отсюда следует, что $\mathbf{L}\left[G_{\leqslant} \mathbf{L}\right]$ является $\mathbb{C}\left(\omega_{1}^{\mathbf{L}}\right)$-ге-

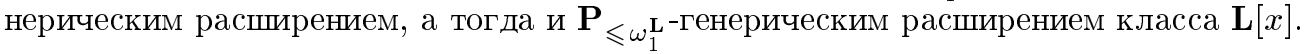
Это дает нам возможность сделать последний шаг. Раз $\mathbf{P}$ изоморфно произведению

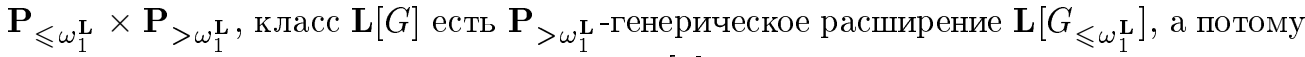

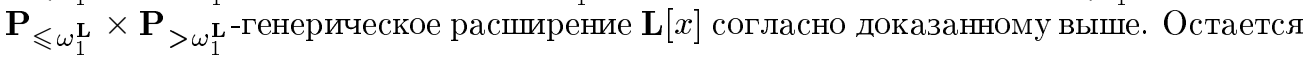
заменить $\mathbf{P}_{\leqslant \omega_{1}^{\mathbf{L}}} \times \mathbf{P}_{>\omega_{1}^{\mathbf{L}}}$ обратно на $\mathbf{P}$.

ЛЕмма 7.13. Предположим, что $x \in \mathbf{L}[G] \cap \mathbb{N}^{\omega}, \varphi\left(v_{1}, \ldots, v_{n}\right)-\in$-формула, $а$ $z_{1}, \ldots, z_{n} \in \mathbf{L}[x]-$ произвольнье множсества. Тогда

$$
\varphi\left(z_{1}, \ldots, z_{n}\right) \text { истинно в } \mathbf{L}[G] \Longleftrightarrow \Lambda \Vdash_{\mathbf{P}}^{\mathbf{L}[x]} \varphi\left(\breve{z}_{1}, \ldots, \breve{z}_{n}\right)
$$

ДокАЗАТЕльство. Рассуждая, как в доказательстве леммы 7.6 (со ссылкой на лемму 7.12 вместо леммы 7.8), вполне достаточно доказать, для любых "условий" $p, q \in \mathbf{P}$, что, в упрощающем предположении $\operatorname{dom} p=\operatorname{dom} q=u \subseteq \omega_{2}^{\mathbf{L}}$ и $\operatorname{dom} p(\xi)=$ $\operatorname{dom} q(\xi)$ для всех $\xi \in u$, множества $\mathbf{P}_{\leqslant p}=\left\{p^{\prime} \in \mathbf{P}: p^{\prime} \leqslant p\right\}$ и $\mathbf{P}_{\leqslant q}$ порядково изоморфны в L. Построение искомого изоморфизма не составляет труда.

7Д. Свойство совершенного ядра во второй модели. Продолжая доказательство теоремы 7.9, мы докажем, что в $\mathbf{P}$-генерической модели $\mathbf{L}[G]$ вьполнено $\mathrm{PK}(\mathrm{OD})$. С этой целью рассмотрим произвольное множество $X \in \mathbf{L}[G], X \subseteq \mathbb{N}^{\omega}$, являюшееся OD множеством в $\mathbf{L}[G]$. Пусть $X=\{x: \varphi(x)\}$ в $\mathbf{L}[G]$, где, для простоты, $\varphi$ - вообще беспараметрическая формула. Обозначим через $\psi(x)$ формулу: “в $\mathbf{L}[x]$ истинно $\Lambda \Vdash_{\mathbf{P}}^{\mathbf{L}[x]} \varphi(\breve{x})$ "; тогда, по лемме 7.13 , мы имеем $\forall x(\varphi(x) \Longleftrightarrow \psi(x))$ в $\mathbf{L}[G]$, так что $X=\{x: \psi(x)\}$.

Если $X \subseteq \mathbf{L}$, то $X$ не более чем счетно в $\mathbf{L}[G]$, поскольку $\omega_{1}^{\mathbf{L}}$ счетно в $\mathbf{L}[G]$. Остается доказать, предполагая $X \nsubseteq \mathbf{L}$, что $X$ имеет совершенное подмножество в $\mathbf{L}[G]$. Пусть $x \in X \backslash \mathbf{L}$. Мы имеем $x \in \mathbf{L}\left[G_{<\vartheta}\right]$ для некоторого $\vartheta<\omega_{2}^{\mathbf{L}}$ по лемме 7.11. Как мы видели в доказательстве леммы $7.13, \mathbf{P}_{<\vartheta}$-генерические и $\mathbb{C}\left(\omega_{1}^{\mathbf{L}}\right)$-генерические (где $\left.\mathbb{C}\left(\omega_{1}^{\mathbf{L}}\right)=\operatorname{Coll}\left(\mathbb{N}, \omega_{1}^{\mathbf{L}}\right)\right)$ расширения - это одно и то же, откуда следует, что найдется $\mathbb{C}\left(\omega_{1}^{\mathbf{L}}\right)$-генерическое над $\mathbf{L}$ множество $F_{0} \subseteq \mathbb{C}\left(\omega_{1}^{\mathbf{L}}\right), F_{0} \in \mathbf{L}[G]$ такое, что $x \in \mathbf{L}\left[F_{0}\right]$. Фиксируем $\mathbb{C}\left(\omega_{1}^{\mathbf{L}}\right)$-имя $t \in \mathbf{L}$ с $x=t\left[F_{0}\right]$. Поскольку формула $\psi$, очевидно, абсолютна, найдется "условие" $p_{0} \in F_{0}$, вьнуждающее $\psi(t) \wedge t \notin \mathbf{L}$. Будем, для краткости, 
считать, что $p_{0}=\Lambda$, т.е. $\Lambda \Vdash_{\mathbb{C}\left(\omega_{1}^{\mathbf{L}}\right)}^{\mathbf{L}} \psi(t) \wedge t \notin \mathbf{L} ;$ обший случай требует лишш простых и очевидных поправок. В этом предположении

$(*) t[F] \in X$ для любого $\mathbb{C}\left(\omega_{1}^{\mathbf{L}}\right)$-генерического над $\mathbf{L}$ множества $F \subseteq \mathbb{C}\left(\omega_{1}^{\mathbf{L}}\right), F \in$ $\mathbf{L}[G]$.

Идея нижеследуюшего построения состоит в том, что мы определим, в $\mathbf{L}[G]$, “совершенное множество" генерических подмножеств $\mathbb{C}\left(\omega_{1}^{\mathbf{L}}\right)$, на котором отображение $F \mapsto$ $t[F]$ будет непрерьвным и взаимно однозначным.

Для $p, q \in \mathbb{C}\left(\omega_{1}^{\mathbf{L}}\right)$ определим $p \perp q$, когда найдутся числа $n$ и $k \neq \ell$ такие, что $p \Vdash_{\mathbb{C}\left(\omega_{1}^{\mathbf{L}}\right)}^{\mathbf{L}} t(\breve{n})=\breve{k}$ и $q \Vdash_{\mathbb{C}\left(\omega_{1}^{\mathbf{L}}\right)}^{\mathbf{L}} t(\breve{n})=\breve{\ell}$. Докажем следуюшее:

$(\dagger)$ если $p, q \in \mathbb{C}\left(\omega_{1}^{\mathbf{L}}\right)$, то найдутся “условия” $p^{\prime} \leqslant p$ и $q^{\prime} \leqslant q$ (т.е. более сильные) такие, что $p^{\prime} \perp q^{\prime}$.

В самом деле, иначе никакие два $p^{\prime}, p^{\prime \prime} \leqslant p$ не могут вынуждать элементарно несовместимых свойств $t$, т.е., например, $p^{\prime} \Vdash_{\mathbb{C}\left(\omega_{1}^{\mathbf{L}}\right)}^{\mathbf{L}} t(\breve{n})=\breve{k}$ и $p^{\prime \prime} \Vdash_{\mathbb{C}\left(\omega_{1}^{\mathbf{L}}\right)}^{\mathbf{L}} t(\breve{n})=\breve{\ell}$ для каких-либо $n$ и $k \neq \ell$. Отсюда следует, что $p$ уже "решает" все значения $t$, другими словами, для любого $n$ найдется $k_{n}$ такое, что $p \Vdash_{\mathbb{C}\left(\omega_{1}^{\mathbf{L}}\right)}^{\mathbf{L}} t(\breve{n})=\breve{k}_{n}$. Но тогда "условие" $p$ вынуждает $t=\breve{a}$, где $a(n)=k_{n}, \forall n$, так что $a \in \mathbf{L}$, поскольку $\Vdash_{\mathbb{C}\left(\omega_{1}^{\mathbf{L}}\right)}^{\mathbf{L}}$ выразимо в исходной модели $\mathbf{L}$. Мы пришли к противоречию с предположением, что $\Lambda \Vdash t \notin \mathbf{L}$, доказывающему $(\dagger)$.

Рассмотрим вспомогательньй форсинг П, состояший из функций $\pi$ таких, что dom $\pi$ - подмножество $2^{<\omega}$ вида $2^{<m}=\left\{s \in 2^{<\omega}: m>\operatorname{lh} s\right\}$, где $m \in \mathbb{N}$ будет обозначаться через $m=\operatorname{hgt} \pi$ (высота $\pi$ ), a $\operatorname{ran} \pi \subseteq \omega_{1}^{\mathrm{L}}$. Упорядочим П обратно включению: $\pi \leqslant \rho(\pi$ сильнее, чем $\rho)$, когда $\operatorname{dom} \rho \subseteq \operatorname{dom} \pi$ (тогда, очевидно, hgt $\rho \leqslant \operatorname{hgt} \pi)$ и $\rho=\pi\lceil\operatorname{dom} \rho$. Ясно, что $\varnothing$ (т.е. пустая функция, то же, что и $\Lambda$ ) является максимальным элементом П.

Форсинг П можно назвать разветвленной степенью $\mathbb{C}\left(\omega_{1}^{\mathbf{L}}\right):$ в самом деле, если $\pi \in \Pi, m=\operatorname{hgt} \pi, s \in 2^{<m}$, то мы можем определить $\pi / s \in \mathbb{C}\left(\omega_{1}^{\mathbf{L}}\right)$ так, что $\operatorname{dom}(\pi / s)=1+\operatorname{lh} s$ и $(\pi / s)(k)=\pi(s\lceil k), \forall k \leqslant \operatorname{lh} s$. Например, если $s=\langle i, j\rangle$, то $\operatorname{dom}(\pi / s)=3,(\pi / s)(0)=\pi(\Lambda),(\pi / s)(1)=\pi(\langle i\rangle),(\pi / s)(2)=\pi(s)$. В то же время $\Pi \in \mathbf{L}$ и $\Pi$ - дерево высоты $\omega$ с $\omega_{1}^{\mathbf{L}}$-ветвлениями (в смысле порядка, совпадающего c $\subseteq$, т.е. обратного $\leqslant)$, так что П и $\mathbb{C}\left(\omega_{1}^{\mathbf{L}}\right)$ порядково изоморфны в $\mathbf{L}$.

Мы утверждаем, что если $D \in \mathbf{L}-$ плотное подмножество $\mathbb{C}\left(\omega_{1}^{\mathbf{L}}\right)$ и $k \in \mathbb{N}$, то следующие множества принадлежат $\mathbf{L}$ и плотны в П:

$$
\begin{aligned}
\Delta_{D} & =\left\{\pi \in \Pi: \forall s \in{ }^{n} 2(\pi / s \in D), \text { где } n=\operatorname{hgt} \pi-1\right\} ; \\
\Delta_{k} & =\left\{\pi \in \Pi: k \leqslant n=\operatorname{hgt} \pi-1 \wedge \forall s, s^{\prime} \in{ }^{n} 2\left(s\left\lceil k \neq s^{\prime}\left\lceil k \Longrightarrow \pi / s \perp \pi / s^{\prime}\right)\right\} .\right.\right.
\end{aligned}
$$

(Здесь ${ }^{n} 2=\left\{s \in 2^{<\omega}: n=\mathrm{lh} s\right\}$.) Для доказательства плотности $\Delta_{D}$, пусть $\rho \in \Pi$, hgt $\rho=m+1 \geqslant 1$. В силу плотности $D$ для всякого $\sigma \in m_{2}$ найдется "условие" $p_{\sigma} \in D$ такое, что $p_{\sigma} \leqslant \rho / \sigma$ в $\mathbb{C}\left(\omega_{1}^{\mathrm{L}}\right)$, т.е. $\rho / \sigma \subseteq p_{\sigma}$. В силу конечности множества $m_{2}$ можно, не ограничива общности, считать, что все $p_{\sigma}$ имеют одно и то же значение $\operatorname{dom} p_{\sigma}=n+1>m$. Теперь не составляет труда определить "условие" $\pi \in \Pi$ так, чтобы hgt $\pi=n+1$ и $\pi / s=p_{s \uparrow m} \in D, \forall s \in{ }^{n} 2$, так что $\pi \in \Delta_{D}$.

Для проверки плотности $\Delta_{k}$, пусть снова $\rho \in \Pi$, hgt $\rho=m+1 \geqslant 1$. Можно считать, что $m \geqslant k$. Используя утверждение $(\dagger)$, мы можем подобрать $p_{\sigma} \in \mathbb{C}\left(\omega_{1}^{\mathbf{L}}\right)$ ко всякому $\sigma \in{ }^{m} 2$ так, что будет $p_{\sigma} \perp p_{\sigma^{\prime}}$ при $\sigma \neq \sigma^{\prime}$. Опять можно считать, что $\operatorname{dom} p_{\sigma}=n+1$, 
где $n>m$, для всех $\sigma \in{ }^{m} 2$. Найдется “условие" $\pi \in \Pi$ такое, что $\operatorname{hgt} \pi=n+1$ и $\pi / s=p_{s \uparrow m}$ для всех $s \in{ }^{n} 2$. Если $s, s^{\prime} \in{ }^{n} 2$ и $s \uparrow k \neq s^{\prime} \uparrow k$, то тем более $s \uparrow m \neq s^{\prime} \uparrow m$, так что $\pi / s=p_{s \uparrow m} \perp p_{s^{\prime} \uparrow m}=\pi / s^{\prime}$. Итак, $\pi \in \Delta_{k}$.

В обоих версиях мы имеем $\pi \leqslant \rho$, что и доказьвает плотность $\Delta_{D}$ и $\Delta_{k}$.

Как было указано вьше, множества $\mathbb{C}\left(\omega_{1}^{\mathbf{L}}\right)$ и П порядково изоморфны в $\mathbf{L}$. А поскольку, как мы видели, $\mathbf{L}[G]$ содержит $\mathbb{C}\left(\omega_{1}^{\mathbf{L}}\right)$-генерическое над $\mathbf{L}$ множество, то в $\mathbf{L}[G]$ сушествует и П-генерическое над $\mathbf{L}$ множество $\Gamma \subseteq \Pi$; тогда $\gamma=\bigcup \Gamma-$ отображение $2^{<\omega} \rightarrow \omega_{1}^{\mathrm{L}}$. Положим $\Gamma / u=\{\pi /(u\lceil n): \pi \in \Gamma \wedge n<\operatorname{hgt} \pi\}$ для каждого $u \in 2^{\omega}$; тогда $\Gamma / u \subseteq \mathbb{C}\left(\omega_{1}^{\mathbf{L}}\right)$. Утверждается, что следуюшее истинно в $\mathbf{L}[G]$ :

(a) при любом $u \in 2^{\omega}$ множество $\Gamma / u$ является $\mathbb{C}\left(\omega_{1}^{\mathbf{L}}\right)$-генерическим над $\mathbf{L}$;

(б) если $u \neq v \in 2^{\omega}$, то $t[\Gamma / u] \neq t[\Gamma / v]$;

(в) отображение $u \mapsto t[\Gamma / u]-$ непрерьвная функци $2^{\omega} \rightarrow \mathbb{N}^{\omega}$ с кодом в $\mathbf{L}$;

(г) $t[\Gamma / u] \in X$ при любом $u \in 2^{\omega}$.

Для доказательства (а) предположим, что множество $D \in \mathbf{L}, D \subseteq \mathbb{C}\left(\omega_{1}^{\mathrm{L}}\right)$ плотно в $\mathbb{C}\left(\omega_{1}^{\mathrm{L}}\right)$. Тогда $\Delta_{D}$ плотно в $\Pi$, следовательно, найдется $n \in \mathbb{N}$ такое, что $\pi=$ $\gamma\left\lceil 2^{<n+1} \in \Delta_{D}\right.$. Положим $s=u\left\lceil n\right.$. Тогда $\pi / s \in D$ по определению $\Delta_{D}$. С другой стороны, легко видеть, что $\pi / s \in \Gamma / u$.

(б) доказьвается аналогично, но с использованием множеств $\Delta_{k}$.

(в) Предположим, что $u \in 2^{\omega}$ и $t[\Gamma / u](m)=k$. Согласно (а), найдется "условие" $p \in \Gamma / u$, вынуждающее $t(\breve{m})=\breve{k}$. По построению $t$ имеет вид $\pi /(u\lceil n)$, где $\pi \in \Gamma$ и $n<\operatorname{hgt} \pi$. Тем самым, $p \in \Gamma / v$ и, следовательно, $t[\Gamma / v](m)=k$, всякий раз, когда $v \in \mathbb{N}^{\omega}$ и $v\lceil n=u\lceil n$.

(г) следует из $(\mathrm{a})$ и $(*)$.

Соединяя вместе (б), (в) и (г), мы немедленно получаем, в $\mathbf{L}[G]$, совершенное подмножество $\left\{t[\Gamma / u]: u \in 2^{\omega}\right\}$ множества $X$, что и требовалось.

$\square$ (теорема 7.9 и теорема 7.1 - часть (I) и теорема в иелом)

ЗАмечАниЕ 7.14. Рассуждая аналогично доказательству теоремы 7.5 вьше,можно доказать, что в Р-генерической модели $\mathbf{L}[G]$ выполнены LM(OD), BP(OD). C другой стороны, методы, о которых пойдет речь в $\S 8$, дают $\neg \operatorname{LM}\left(\boldsymbol{\Delta}_{2}^{1}\right)$ и $\neg \operatorname{BP}\left(\boldsymbol{\Sigma}_{2}^{1}\right)$, но $\operatorname{BP}\left(\boldsymbol{\Delta}_{2}^{1}\right)$, по той причине, что, для любого $a \in \mathbf{L}[G] \cap \mathbb{N}^{\omega} \mathrm{c} \omega_{1}^{\mathbf{L}[a]}=\omega_{2}^{\mathbf{L}}$, множество Coh L $[a]$ непусто, но не является котошим, a Rand L $[a]=\varnothing$.

Отсюда следует, что конъюнкция $\mathrm{PK}(\mathrm{OD}), \mathrm{LM}(\mathrm{OD}), \mathrm{BP}(\mathrm{OD})$ совместима с $\neg \operatorname{PK}\left(\boldsymbol{\Pi}_{1}^{1}\right) \wedge \neg \operatorname{LM}\left(\Delta_{2}^{1}\right) \wedge \neg \operatorname{BP}\left(\Sigma_{2}^{1}\right)$.

Мы предполагаем, что здесь $\neg \mathrm{BP}\left(\boldsymbol{\Sigma}_{2}^{1}\right)$ может быть улучшено до $\neg \mathrm{BP}\left(\boldsymbol{\Delta}_{2}^{1}\right)$. Однако наиболее интересный вопрос, на который мы не можем ответить, состоит в следующем: вьполняется ли $\mathrm{PK}(\mathrm{OD})$ в $\mathbb{C}\left(\omega_{1}^{\mathrm{L}}\right)$-генерической модели, рассмотренной вьше в п. 7Б? Вообще, совместимо ли $\mathrm{PK}(\mathrm{OD})$ с предположением, что $\mathbb{N}^{\omega} \subseteq \mathbf{L}[a]$ для некоторого $a \in \mathbb{N}^{\omega}$ ?

7Е. Модель Соловея. Здесь мы начинаем доказательство теоремы 7.2. Форсинг, который будет использован в доказательстве, являетс произведением "склеивающих" форсингов вида $\operatorname{Coll}(\mathbb{N}, \xi)$, однако, в отличие от форсинга $\mathbf{P}$ из п. 7Г, где все сомножители имели одно и то же значение $\xi=\omega_{2}^{\mathrm{L}}$, в этом новом варианте $\xi$ будет меняться от 0 до фиксированного строго недостижимого кардинала в исходной модели.

Перейдем к деталям. Фиксируем строго недостижимьй в классе $\mathbf{L}$ конструктивных множеств кардинал $\Omega$. Рассмотрим форсинг $\mathscr{P}$, состояший из всех функций $p$ 
таких, что $\operatorname{dom} p \subseteq \Omega$ конечно, а $p(\xi) \in \operatorname{Coll}(\mathbb{N}, \xi)$, т.е. $p$ - функция из некоторого $m=\{0, \ldots, m-1\}$ в $\xi=\{\eta: \eta<\xi\}$ для всех $\xi \in \operatorname{dom} p$. Упорядочим $\mathscr{P}$ аналогично $\mathbf{P}$ выше, а именно, $p \leqslant q$ ( $p$ является более сильным условием), когда $\operatorname{dom} q \subseteq \operatorname{dom} p$ и $p(\xi) \leqslant q(\xi)$ (т.е. $q(\xi)=p(\xi)\lceil\operatorname{dom} q(\xi)$ ) для всех $\xi \in \operatorname{dom} p$. Как само $\mathscr{P}$, так и этот порядок принадлежат $\mathbf{L}$. Положим

$$
\mathscr{P}_{<\vartheta}=\{p \in \mathscr{P}: \operatorname{dom} p \subseteq \vartheta\}, \quad \mathscr{P}_{\geqslant \vartheta}=\{p \in \mathscr{P}: \operatorname{dom} p \subseteq \Omega \backslash \vartheta\}
$$

а также $\mathscr{P}_{\leqslant \vartheta}=\mathscr{P}_{<\vartheta+1}$ и $\mathscr{P}_{>\vartheta}=\mathscr{P}_{\geqslant \vartheta+1}$.

Подобно теореме 7.1, теорема 7.2 вытекает из следующего утверждения.

Teорема 7.15. Если множество $\mathscr{G} \subseteq \mathscr{P}$ является $\mathscr{P}$-генерическим над $\mathbf{L}$, то в L[G] виполнены $\mathrm{PK}(\mathbf{R O D}), \mathrm{LM}(\mathbf{R O D}), \mathrm{BP}(\mathbf{R O D})$.

Доказывая эту теорему, фиксируем $\mathscr{P}$-генерическое над $\mathbf{L}$ множество $\mathscr{G} \subseteq \mathscr{P}$. Положим $\mathscr{G}_{<\vartheta}=\mathscr{G} \cap \mathscr{P}_{<\vartheta}$ и аналогично $\mathscr{G}_{\geqslant \vartheta}, \mathscr{G}_{\leqslant \vartheta}, \mathscr{G}_{>\vartheta}$.

Начнем с ключевых технических фактов о модели Соловея $\mathbf{L}[\mathscr{G}]$.

Лемма 7.16. Любая антицепь $A \subseteq \mathscr{P}, A \in \mathbf{L}$ имеет мощность $<\Omega$ в $\mathbf{L}$.

ДоказАтельство. Рассуждаем в $\mathbf{L}$. Можно считать, что $A$-максимальная антицепь. Положим $\xi_{0}=\omega$. Если $\xi_{n}<\Omega$ определено, то множество $\mathscr{P}_{<\xi_{n}}$ имеет, очевидно, мошность $<\Omega$, а потому, вследствие недостижимости $\Omega$ и максимальности $A$, найдется ординал $\xi, \xi_{n}<\xi<\Omega$, такой, что для всякого $p \in \mathscr{P}_{<\xi_{n}}$ существует "условие" $q \in A \cap \mathscr{P}_{<\xi}$, совместимое с $p$ в $\mathscr{P}$. Наименьший из таких ординалов $\xi$ обозначим через $\xi_{n+1}$. Положим $\vartheta=\sup _{n} \xi_{n} ;$ снова $\vartheta<\Omega$ из-за недостижимости $\Omega$. Мы имеем $A \subseteq \mathscr{P}_{<\vartheta}$ (см. доказательство леммы 7.10), откуда и вытекает искомый результат, поскольку множество $\mathscr{P}<\vartheta$ имеет мошность $\operatorname{card} \vartheta<\Omega$.

СледСТвиЕ 7.17. Выполняется $\omega_{1}^{\mathbf{L}[\mathscr{G}]}=\Omega$.

ДокАЗАТЕЛЬСтво. Неравенство $\omega_{1}^{\mathbf{L}[\mathcal{G}]} \leqslant \Omega$ следует из леммы 7.16 и теоремы 4.2(i). Обратно, каждое множество $\mathscr{G}_{\xi}=\{p(\xi): p \in \mathscr{G}\}$ является $\operatorname{Coll}(\mathbb{N}, \xi)$-генерическим над $\mathbf{L}$ (по теореме $4.2(\mathrm{iii})$, так что $f_{\xi}[\mathscr{G}]=\bigcup \mathscr{G}_{\xi}$ - функция из $\mathbb{N}$ на $\xi$, откуда следует, что $\xi<\omega_{1}^{\mathbf{L}[\mathscr{G}]}$. Раз $\xi<\Omega$ произвольно, мы имеем $\Omega \leqslant \omega_{1}^{\mathbf{L}[\mathscr{G}]}$.

Лемма 7.18. Если $x \in \mathbf{L}[\mathscr{G}] \cap \mathbb{N}^{\omega}$, то найдется ординал $\vartheta<\Omega$ такой, что $x \in \mathbf{L}\left[\mathscr{G}_{<\vartheta]}\right.$, следовательно, $\omega_{1}^{\mathbf{L}[x]}<\Omega=\omega_{1}^{\mathbf{L}[\mathscr{G}]} u \mathbf{L}[a] \cap \mathbb{N}^{\omega}$ счетно в $\mathbf{L}[G]$.

ДокАЗАТЕльство. Сушествование $\vartheta$ доказьвается, как и в лемме 7.11. Далее, отображение $\langle p, q\rangle \mapsto p \cup q-$ порядковый изоморфизм между $\mathscr{P}_{<\vartheta} \times \mathscr{P}_{\geqslant \vartheta}$ и $\mathscr{P}$, откуда нетрудно вывести, используя теорему $4.2(\mathrm{iii})$, что множество $\mathscr{G}_{<\vartheta}$ является $\mathscr{P}<\vartheta$-генерическим над $\mathbf{L}$. Поскольку с $\operatorname{ard} \mathscr{P}_{<\vartheta}<\Omega$ в $\mathbf{L}$, найдется $\varkappa<\Omega$ такое, что в $\mathbf{L}$ истинно: $\varkappa$ - регулярный кардинал и саrd $\mathscr{P}_{<\vartheta}<\varkappa$. Тогда $\omega_{1}^{\mathbf{L}[x]} \leqslant \omega_{1}^{\mathbf{L}\left[\mathscr{G}_{<\vartheta}\right]} \leqslant \varkappa<\Omega$ по теореме 4.2(i), так что $\omega_{1}^{\mathbf{L}[x]}<\Omega=\omega_{1}^{\mathbf{L}[\mathscr{G}]}$.

ЛЕмма 7.19 (ср. с леммами 7.8, 7.12). Если $x \in \mathbf{L}[\mathscr{G}] \cap \mathbb{N}^{\omega}$, mо класс $\mathbf{L}[\mathscr{G}]$ является $\mathscr{P}$-генерическим расширением $\mathbf{L}[x]$. 
ДокАЗАТЕЛЬство (набросок). Лемма 7.18 приносит ординал $\vartheta<\Omega$ такой, что $x \in \mathbf{L}\left[\mathscr{G}_{<\vartheta}\right]$. Можно считать, что $\vartheta=\varkappa+1$, где $\varkappa<\Omega-$ кардинал в L. Множество $\mathscr{P}$ в всех “условий” $p \in \mathscr{P} \leqslant \varkappa$ таких, что $\xi \in \operatorname{dom} p, \operatorname{dom} p \backslash\{\xi\}=\operatorname{ran} p(\xi)$ и $\operatorname{dom} p(\eta)=\operatorname{dom} p(\xi)$ для всех $\eta \in \operatorname{dom} p$, плотно в $\mathscr{P} \leqslant \varkappa$ и представляет собой дерево с $\varkappa$-ветвлениями (так как $\varkappa$ - кардинал). Поэтому $\mathscr{P}^{\prime}$ порядково изоморфно множеству $\mathbb{C}(\varkappa)=\operatorname{Coll}(\mathbb{N}, \varkappa)$ в $\mathbf{L}$, откуда следует, что $\mathbf{L}\left[\mathscr{G}_{\leqslant} \varkappa\right]=\mathbf{L}[F]$, где множество $F \subseteq \mathbb{C}(\varkappa)$ является $\mathbb{C}(\varkappa)$-генерическим над $\mathbf{L}$. Аналогично доказательству леммы 7.8, найдется дерево $\Sigma \subseteq \mathbb{C}(\varkappa), \Sigma \in \mathbf{L}[x]$ без $\subseteq$-максимальных элементов такое, что $F \subseteq \Sigma$ и $F$ является $\Sigma$-генерическим над $\mathbf{L}[x]$. Далее нужно следовать доказательству леммы 7.12 , в частности, использовать тот очевидньй факт, что множество $\mathbb{C}(\vartheta)=\operatorname{Coll}(\mathbb{N}, \vartheta)$ порядково изоморфно $\Sigma \times \mathbb{C}(\vartheta)$, вследствие чего оказывается, что $\left.\mathbf{L}\left[\mathscr{G}_{\leqslant}\right\}\right]$есть $\mathbb{C}(\vartheta)$-генерическое расширение класса $\mathbf{L}[a]$.

Лемма 7.20. Предположим, что $x \in \mathbf{L}[\mathscr{G}] \cap \mathbb{N}^{\omega}, \varphi\left(v_{1}, \ldots, v_{n}\right)-\in$-формула, $а$ $z_{1}, \ldots, z_{n} \in \mathbf{L}[x]-$ произвольные множества. Тогда

$$
\varphi\left(z_{1}, \ldots, z_{n}\right) \text { истинно в } \mathbf{L}[\mathscr{G}] \Longleftrightarrow \Lambda \Vdash_{\mathscr{P}}^{\mathbf{L}[x]} \varphi\left(\breve{z}_{1}, \ldots, \breve{z}_{n}\right)
$$

ДокАЗАТЕЛЬСтво. См. доказательство леммы 7.13.

ДОКАЗАТЕЛЬСТВО ТЕОРЕМЫ 7.15. Начнем с измеримости и свойства Бэра в модели Соловея $\mathbf{L}[\mathscr{G}]$. Пусть $a \in \mathbf{L}[\mathscr{G}] \cap \mathbb{N} \omega$. Рассмотрим, рассуждая в $\mathbf{L}[\mathscr{G}]$, произвольньй $\mathbf{L}$-абсолютньй $\sigma$-УСА-идеал $\mathscr{I}$ борелевских подмножеств $\mathbb{N}^{\omega}$ и $\mathbf{R O D}$-множество $X \subseteq \mathbb{N}^{\omega}$. Требуется доказать, что множество $X \quad \mathscr{I}$-измеримо.

Можно считать, что $X$ принадлежит $\mathrm{OD}(a)$ (в противном случае просто присоединяем к $a$ все параметры из $\mathbb{N}^{\omega}$, участвуюшие в определении $\left.X\right)$. Тогда $X=\{x$ : $\varphi(a, x)\}$, где $\varphi$ содержит лишь $a$ и ординалы в качестве параметров. Считая, для простоты, что на самом деле $a$ - единственный параметр $\varphi$ (общий случай вполне аналогичен), мы имеем, по лемме $7.20, X=\{x: \psi(a, x)\}$, где $\psi(a, x)$ есть формула "в $\mathbf{L}[a, x]$ истинно $\Lambda \Vdash_{\mathscr{P}}^{\mathbf{L}[a, x]} \varphi(\breve{a}, \breve{x}) "$. Из леммы 7.18 следует, что множество $\operatorname{Rand}_{\mathscr{I}} \mathbf{L}[a]$ является $\mathscr{I}$-полным. В этой ситуации свойство $\mathscr{I}$-измеримости $X$ в L $[\mathscr{G}]$ следует из теоремы 4.17 .

Теперь о свойстве совершенного ядра. Рассмотрим, в L[ $\mathscr{G}], \mathbf{R O D}$-множество $X=$ $\{x: \varphi(a, x)\}=\{x: \psi(a, x)\} \subseteq \mathbb{N}^{\omega}$, где $a, \varphi, \psi$ таковы, как указано вьше. Предполагая $X$ несчетным в $\mathbf{L}[\mathscr{G}]$, мы имеем $X \nsubseteq \mathbf{L}[a]$. Фиксируем $x \in X \backslash \mathbf{L}[a]$. Согласно лемме $7.18 a, x \in \mathbf{L}\left[\mathscr{G}_{<\vartheta}\right]$ для подходящего $\vartheta<\Omega$. При этом класс $\left.\mathbf{L}\left[\mathscr{G}_{\leqslant}\right\}\right]$является $\mathbb{C}(\vartheta)$-генерическим расширением $\mathbf{L}[a]$, где $\mathbb{C}(\vartheta)=\operatorname{Coll}(\mathbb{N}, \vartheta)$ (см. доказательство леммы 7.19), т.е. найдется $\mathbb{C}(\vartheta)$-генерическое над $\mathbf{L}[a]$ множество $F_{0} \subseteq \mathbb{C}(\vartheta)$ такое, что $\mathbf{L}\left[\mathscr{G}_{\leqslant \vartheta}\right]=\mathbf{L}[a][F]$. При этом $x \in \mathbf{L}[a][F] \backslash \mathbf{L}[a]$. Дальнейший ход рассуждений повторяет выкладки п. 7 Д, с той разницей, что $\mathbf{L}[a]$ всюду заменяет $\mathbf{L}$.

$\square$ (теоремы 7.15 и 7.2$)$

Исторические и библиографические замечания. Мы ссылаемся на [75] или [29; разд. 14] в отношении понятий, связанных с ординальной определимостью.

Теорема 7.2 и следствие 7.3 из нее принадлежат Соловею [89] на русском языке (несколько от личающиеся в деталях доказательства можно найти в [29], [35]). Модель $\mathbf{L}[\mathscr{G}]$, являюшаяся предметом теоремы 7.15, принято назьвать моделью Соловея, но первая публикащия о ней принадлежит Леви [49]. 
Часть (ii) следствия 7.3 и следствие 7.4 в частях (ii) и (iii), а также (в ослабленной форме, см. ниже о теореме 7.1) и в части (i) - о неразрешимости классических проблем из диаграммы во введении независимо получена Любецким [17], [68]. Теорема 7.1 в части (II) приведена в [65], [68], [69]. Теорема 7.1 в части (III) вполне аналогична. Доказательство в части (I) получено в ходе написания этой статьи, а более слабый результат, о том, что в $\mathbf{Z F C}$ из $\neg \mathrm{PK}\left(\boldsymbol{\Pi}_{1}^{1}\right)$ не следует сушествование OD решета, определяющего несчетное $\boldsymbol{\Pi}_{1}^{1}$-множество без совершенных подмножеств, приведен Любецким в [17], [67] (доказательство дано в [68]). Ключевая лемма 7.19 доказана Соловеем [89]; леммы 7.8, 7.12 представляют собой вариации на ту же тему.

\section{§ 8. Необратимость импликаций}

Вернемся к нашей диаграмме из введения. Является ли полной картина представленных на ней связей между пятью рассмотренными там предложениями? Следующая теорема дает положительный ответ на этот вопрос.

Tеорема 8.1. В ZFC не вьводится ни одна из следующих импликачий:

(1) $\operatorname{LM}\left(\boldsymbol{\Sigma}_{2}^{1}\right) \Longrightarrow \operatorname{PK}\left(\bar{\Pi}_{1}^{1}\right) ; \quad(2) \quad \operatorname{BP}\left(\boldsymbol{\Sigma}_{2}^{1}\right) \wedge \operatorname{LM}\left(\boldsymbol{\Delta}_{2}^{1}\right) \Longrightarrow \operatorname{LM}\left(\boldsymbol{\Sigma}_{2}^{1}\right) ;$

(3) $\mathrm{BP}\left(\boldsymbol{\Sigma}_{2}^{1}\right) \Longrightarrow \operatorname{LM}\left(\boldsymbol{\Delta}_{2}^{1}\right) ; \quad$ (4) $\mathrm{BP}\left(\boldsymbol{\Delta}_{2}^{1}\right) \wedge \operatorname{LM}\left(\boldsymbol{\Delta}_{2}^{1}\right) \Longrightarrow \mathrm{BP}\left(\boldsymbol{\Sigma}_{2}^{1}\right)$;

(5) $\operatorname{LM}\left(\boldsymbol{\Delta}_{2}^{1}\right) \Longrightarrow \operatorname{BP}\left(\boldsymbol{\Delta}_{2}^{1}\right)$.

Доказательства всех пяти частей этой теоремы могут быть проведены по единой схеме, основанной на итерациях форсинга. При этом длина итерации составляет $\omega_{1}, \mathrm{a}$ итерируемые форсинги берутся из списка, включающего четыре элементарных форсинга, рассматриваемых в п. 8А. Наиболее длинные и технически сложные рассуждения используются в доказательствах невьводимости импликаций (2) и (4), в частности, вследствие того, что в этих случаях итерации носят смешанньй характер. Здесь, а также в доказательстве невьводимости импликации (3), мы ссылаемся на результаты $\oint 5$ о связи свойств регулярности со свойствами отношения $\leqslant *$ на $\mathbb{N}^{\omega}$ и $\ell_{1}$. Доказательство невыводимости (5) отличается от других доказательств тем, что требует итерацию со счетной, а не конечной поддержкой.

Изложение в этом параграфе предполагает некоторое знакомство читателя с итерированным форсингом (ссылки о нем см. ниже в исторических и библиографических замечаниях).

8А. Четыре ключевых форсинга. Доказательство теоремы 8.1 использует генерические модели, связанные со следуюшими четырьмя форсингами.

форсинг Коэна: $\mathbf{C}=\mathbb{N}<\omega$, с порядком $s \leqslant t(s$ сильнее), если $t \subseteq s$;

доминирующий форсинг: $\mathbf{D}$ состоит из всех пар вида $\langle s, f\rangle$ таких, что $s \in \mathbb{N}<\omega$, $f \in \mathbb{N}^{\omega}, s \subset f$, с порядком $\langle s, f\rangle \leqslant\langle t, g\rangle(\langle s, f\rangle$ сильнее), если $t \subseteq s$ и $\forall n(g(n) \leqslant f(n))$;

случайный форсинг: В состоит из всех деревьев $T \subseteq 2^{<\omega}$ (см. п. $\left.1 \mathrm{~A}\right)$, без $\subseteq$-максимальных элементов, таких, что $\boldsymbol{\lambda}([T])>0$, с порядком $T \leqslant S$ ( $T$ сильнее), если $T \subseteq S$

$\varepsilon$-случайный форсинг: если $0<\varepsilon<1$, то $\mathbf{A}_{\varepsilon}$ состоит из всех деревьев $T \in \mathbf{B}$ таких, что $\boldsymbol{\lambda}([T])>\varepsilon$, с тем же порядком, что и $\mathbf{B}$.

Понятно, что $\mathbf{C}$ - счетное множество, одно и то же во всех моделях. В то же время, если $\mathfrak{M}$ - транзитивная модель $\mathbf{Z F C}$, то $(\mathbf{D})^{\mathfrak{M}}$ (т.е. $\mathbf{D}$, определенное в $\mathfrak{M}$ ) совпадает с $\mathbf{D} \cap \mathfrak{M}$ по теореме абсолютности 2.8 , и то же для $\mathbf{B}$ и $\mathbf{A}_{\varepsilon}$. Обычно принято говорить: $\mathbf{D}$-генерическое над $\mathfrak{M}$ множество или $\mathbf{D}$-генерическое расширение $\mathfrak{M}$, имея в виду, 
конечно, $(\mathbf{D})^{\mathfrak{M}}$-генерические множества и расширения (вообще, $\mathbf{D} \in \mathfrak{M}$ означает, что $\left.\mathbb{N}^{\omega} \subseteq \mathfrak{M}\right),-$ и мы будем придерживаться этой практики. То же для $\mathbf{B}$ и $\mathbf{A}_{\varepsilon}$.

Всякое $\mathbf{C}$-генерическое множество $G \subseteq \mathbf{C}$ порождает точку $a_{G}=\bigcup G \in \mathbb{N} \omega$, являющуюся коэновской над исходной моделью (п. $4 \Gamma$ ).

D-генерические множества $G$ также порождают точки $\mathbb{N}^{\omega}$ : положим $a_{G}=\bigcup\{s$ : $\exists f(\langle s, f\rangle \in G)\}$. Вторые компоненты $f$ “условий" $\langle s, f\rangle$ играют роль ограничителей снизу для $a_{G}$ : понятно, что $a_{G}(n) \geqslant f(n)$ всякий раз, когда $\langle s, f\rangle \in G$ и $n \geqslant$ lh $s$. Отсюда легко следует, что $f \leqslant{ }^{*} a_{G}$ для любой функции $f \in \mathbb{N}^{\omega}$ из исходной модели, где $\leqslant^{*}$ - отношение финального доминирования, т.е. $f \leqslant{ }^{*} g$, если $f(n) \leqslant g(n)$ для почти всех (кроме конечного числа) $n \in \mathbb{N}$.

Если множество $G \subseteq \mathbf{B}$ является генерическим, то $T_{G}=\bigcap G$ представляет собой ветвь в $2^{<\omega}$, другими словами, сушествует единственная точка $a_{G} \in 2^{\omega}$, принадлежашая пересечению $[G]=\bigcap_{T \in G}[T]$. Точка $a_{G} \quad \lambda$-случайна над исходной моделью согласно следствию 4.14 .

Наконец, $\mathbf{A}_{\varepsilon}$-генерическое множество $G$ порождает дерево $T_{G}=\bigcap G \subseteq 2^{<\omega}$, причем замкнутое множество $\left[T_{G}\right] \subseteq 2^{\omega}$ удовлетворяет $\lambda\left(\left[T_{G}\right]\right)=\varepsilon$, а каждая его точка случайна над исходной моделью (п. 8Б).

\section{Лемма 8.2. Каждый из четьрех форсингов удовлетворяет УСА.}

ДоказАТЕльство. УСА для $\mathbf{C}$ очевидно: множество счетно. Для $\mathbf{D}$ нужно отметить, что любые два “условия" $\langle s, f\rangle,\langle t, g\rangle$ совместимы в $\mathbf{D}$ при $s=t$. Для $\mathbf{B}$, отметим, что если $T, S \in \mathbf{B}$ несовместны в $\mathbf{B}$, то $\boldsymbol{\lambda}([T] \cap[S])=0$. Наконец, рассмотрим $\mathbf{A}_{\varepsilon}$. Пусть, напротив, $A \subseteq \mathbf{A}_{\varepsilon}$ - несчетная антицеп. Для некоторого $\delta>0$ множество $A^{\prime}=\{T \in A: \boldsymbol{\lambda}([T])>\varepsilon+3 \delta\}$ также несчетно. Каждое $T \in A^{\prime}$ можно накрыть множеством $U_{T}$, представляюшим собой дополнение объединения конечно20 числа бэровских интервалов, с $\boldsymbol{\lambda}\left(U_{T}\right)<\boldsymbol{\lambda}(T)+\delta$. Тогда $\boldsymbol{\lambda}\left(U_{T} \cap U_{T^{\prime}}\right) \leqslant \boldsymbol{\lambda}([T] \cap$ $\left.\left[T^{\prime}\right]\right)+2 \delta \leqslant \varepsilon+2 \delta$, так что $U_{T} \neq U_{T^{\prime}}$ при $T \neq T^{\prime} \in A^{\prime}$, откуда следует, что множество $\left\{U_{T}: T \in A^{\prime}\right\}$ несчетно вместе с $A^{\prime}$ - противоречие, так как имеется лишь счетно много конечных объединений интервалов.

Отметим, что каждый из четырех форсингов допускает эквивалентное (в смысле теоремы 4.2(ii)) представление в виде $\mathbb{P}_{\mathscr{I}}$ для подходящего $\sigma$-УСА-идеала $\mathscr{I}, \mathfrak{M}$-абсолютного для любой транзитивной модели $\mathfrak{M} \models \mathbf{Z F C}$. Для $\mathbf{C}$ и $\mathbf{B}$ подойдут идеалы $\mathscr{I}_{\text {cat }}$ и $\mathscr{I}_{\boldsymbol{\lambda}}($ п. $4 \Gamma)$, для $\mathbf{D}$ подходит идеал тощих множеств в смысле определенной не польской топологии (п. 9В), а относительно $\mathbf{A}_{\varepsilon}$ см. [10; 3.4.B].

8Б. $\operatorname{LM}\left(\boldsymbol{\Sigma}_{2}^{1}\right) \not \operatorname{PK}\left(\Pi_{1}^{1}\right)$. В принципе, это - самая простая часть теоремы 8.1. Можно воспользоваться стантартной моделью - обозначим ее через $\mathbf{L}[X]$ - где вьполнена аксиома Мартина $\mathbf{M} \mathbf{\aleph}_{\aleph_{1}}$ для семейств мошности $\aleph_{1}$, и дополнительно $\omega_{1}^{\mathbf{L}[X]}=\omega_{1}^{\mathbf{L}} .($ См. [11; гл. 5, $\S 6],[73]$ или [29; разд. 22] о построении такой модели.) Из $\omega_{1}^{\mathbf{L}[X]}=\omega_{1}^{\mathbf{L}}$ следует $\neg \mathrm{PK}\left(\boldsymbol{\Pi}_{1}^{1}\right)$ в $\mathbf{L}[X]$ согласно следствию 3.4. С другой стороны, известно, что $\mathbf{M} \mathbf{A}_{\aleph_{1}}$ влечет аддитивность идеала множеств меры 0 относительно объединений в числе $\aleph_{1}$ (см. [73] или [29; теорема 50]), откуда следует $\operatorname{LM}\left(\Sigma_{2}^{1}\right)$ согласно следствию 1.7 .

Теперь мы хотим привести более прямое (хотя и более длинное) рассуждение, которое, в отличие от построения на основе $\mathbf{M} \mathbf{A}_{\aleph_{1}}$, дает модель, в которой верна континуум-гипотеза $2^{\aleph_{0}}=\aleph_{1}$. Заметим, что, согласно следствию 3.4 , для доказательства 
$\operatorname{LM}\left(\boldsymbol{\Sigma}_{2}^{1}\right) \not \operatorname{PK}\left(\boldsymbol{\Pi}_{1}^{1}\right)$ достаточно построить модель, в которой вьполнено $\omega_{1}^{\mathbf{L}[a]}=\omega_{1}$ и $\boldsymbol{\lambda}(\boldsymbol{R} \operatorname{and} \mathbf{L}[a])=1$ для каждого $a \in \mathbb{N}^{\omega}$.

Сначала рассмотрим более элементарную задачу: если $\mathfrak{M}$ - транзитивная модель ZFC, то как построить ее генерическое расширение $\mathfrak{M}[G]$, в котором имеет место $\boldsymbol{\lambda}(\boldsymbol{R a n d} \mathfrak{M})=1$ и $\omega_{1}^{\mathfrak{M}}=\omega_{1}^{\mathfrak{M}[G]}$ ? Эта задача решается при помоши $\varepsilon$-случайного форсинга $\mathbf{A}_{\varepsilon}$, где для определенности мы полагаем $\varepsilon=\frac{1}{2}$.

Лемма 8.3. Пусть множество $G$ является $\left(\mathbf{A}_{\varepsilon}\right)^{\mathfrak{M}}$-генерическим над $\mathfrak{M}$. Тог$\partial a \omega_{1}^{\mathfrak{M}}=\omega_{1}^{\mathfrak{M}[G]}$ u $\boldsymbol{\lambda}(\mathbf{R a n d} \mathfrak{M})=1 \quad$ в $\mathfrak{M}[G]$.

ДокаЗАТЕЛЬство. Понятно, что $[G]=\{[T]: T \in G\}$ - центрированная система замкнутых подмножеств $2^{\omega}$ меры $>\varepsilon$, так что $X=\bigcap[G]$ - также замкнутое подмножество $2^{\omega}$ с $\boldsymbol{\lambda}(X) \geqslant \varepsilon$ - на самом деле, $\boldsymbol{\lambda}(X)=\varepsilon$. Mbl утверждаем, что $\bigcap[G] \subseteq \operatorname{Rand} \mathfrak{M}]$. Действительно, пусть $c \in \mathbf{B C} \cap \mathfrak{M}$ и $\boldsymbol{\lambda}\left(\boldsymbol{B}_{c}\right)=0$. Тогда множество $D_{c}=\left\{T \in\left(\mathbf{A}_{\varepsilon}\right)^{\mathfrak{M}}:[T] \cap \boldsymbol{B}_{c}=\varnothing\right\}$ плотно в $\left(\mathbf{A}_{\varepsilon}\right)^{\mathfrak{M}}$ и принадлежит $\mathfrak{M}$, откуда следует, что $D_{c} \cap G \neq \varnothing$, и далее $\boldsymbol{B}_{c} \cap X=\varnothing$, что и требовалось.

Итак, в $\mathfrak{M}[G]$ истинно, что сушествует замкнутое множество $X=\bigcap[G] \subseteq$ Rand $\mathfrak{M}$ $\lambda$-меры $\varepsilon>0$. Более того, для всякого $\varepsilon^{\prime}>\varepsilon$ найдется $T \in G$ (тогда $X \subseteq[T]$ ) с $\varepsilon<\boldsymbol{\lambda}([T])<\varepsilon^{\prime}$. Отсюда нетрудно вьвести, используя лемму 1.3 , что на самом деле $\lambda($ Rand $\mathfrak{M})=1$ в $\mathfrak{M}[G]$.

$($ лемма 8.3)

Доказательство самой теоремы 8.1(1) состоит в итерировании форсинга $\mathbf{A}_{\varepsilon}$. По обшим теоремам об итерированном форсинге с конечной поддержкой, найдется форсинг $\mathbb{P} \in \mathbf{L}$, удовлетворяюший УCA в $\mathbf{L}$ и производящий генерические объекты вида

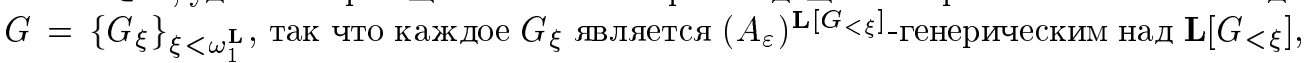
где $G_{<\xi}=\left\{G_{\eta}\right\}_{\eta<\xi}$. Из УСА следует, что $\omega_{1}^{\mathbf{L}[G]}=\omega_{1}^{\mathbf{L}}$, и, кроме того, для каждой точки $a \in \mathbf{L}[G] \cap \mathbb{N}^{\omega}$ найдется ординал $\xi<\omega_{1}^{\mathbf{L}}$ такой, что $a \in \mathbf{L}\left[G_{<\xi}\right] \cap \mathbb{N}^{\omega}$. В этом случае, согласно лемме $8.3, \operatorname{Rand} \mathbf{L}[a]$ - множество полной $\lambda$-меры в $\mathbf{L}[G \leqslant \xi]$, и, следовательно, в $\mathbf{L}[G]$.

$\square($ теорема $8.1(1))$

8B. $\mathrm{BP}\left(\boldsymbol{\Sigma}_{2}^{1}\right) \not \operatorname{LM}\left(\boldsymbol{\Delta}_{2}^{1}\right)$. Здесь используется итерация доминирующего форсинга D. Мы собираемся воспользоваться теоремой 5.3, для чего необходима следующая лемма.

ЛЕМма 8.4. Если $a \in \mathbb{N}^{\omega} u G \subseteq(\mathbf{D})^{\mathbf{L}[a]}$ является $(\mathbf{D})^{\mathbf{L}[a]}$-генерическим над $\mathbf{L}[a], \operatorname{mo}$ в $\mathbf{L}[a, G] \cap \mathbf{C o h} \mathbf{L}[a] \neq \varnothing$.

ДокАЗАТЕЛЬСТво. Положим $y=\bigcup\{s: \exists f(\langle s, f\rangle \in G)\}$. Напомним, что $(m)_{1}=i$ при $m=2^{i}(2 j+1)-1$ для некоторого $j$. Положим $\bar{y}(n)=(y(n))_{1}$, $\forall n$, так что $\bar{y} \in \mathbb{N}^{\omega}$. Мы утверждаем, что $\bar{y} \in \mathbf{C o h} \mathbf{L}[a]$. Достаточно доказать, что $\bar{y} \notin[T]$, каково бы ни было дерево $T \in \mathbf{L}[a], T \subseteq \mathbb{N}<\omega$ такое, что $[T]$ - нигде не плотное подмножество $\mathbb{N}^{\omega}$. (См. доказательство теоремы 5.3.) Если $T$ таково, как указано, то множество $D_{T}$ всех “условий” $\langle s, f\rangle \in(\mathbf{D})^{\mathbf{L}[a]}$, удовлетворяюших $\mathscr{N}_{\bar{s}} \cap[T]=\varnothing$ (где $\bar{s} \in \mathbb{N}^{<\omega}$ получается из $s$ так же, как $\bar{y}$ из $y ; \operatorname{lh} \bar{s}=\operatorname{lh} s$ ), плотно в $(\mathbf{D})^{\mathbf{L}[a]}$ и принадлежит $\mathbf{L}[a]$ вместе с $T$. Поэтому $D_{T} \cap G \neq \varnothing$, откуда и следует искомое.

Доказательство теоремы 8.1(3) состоит в использовании итерации форсинга $\mathbf{D}$ длины $\omega_{1}^{\mathrm{L}}$. Здесь нам, к сожалению, придется войти в технические детали итерированного форсинга с конечной поддержкой. 
Рассуждая в $\mathbf{L}$, мы определяем, индукцией по $\xi, 0<\xi \leqslant \omega_{1}^{\mathbf{L}}$, форсинг $\mathbb{P}_{\xi}$, состоящий из функций $p$, определенных на $\xi$, а также объекты $\overline{\mathbb{Q}}^{\xi}, Q^{\xi}, \mathbf{1}_{\xi}$ :

$1^{\circ} . \mathbb{P}_{0}=\{\Lambda\}$ и $\mathbf{1}_{0}=\Lambda$ (пустая функция).

$2^{\circ}$. Если форсинг $\mathbb{P}_{\xi}$ определен (и состоит из функций $p$ c $\left.\operatorname{dom} p=\xi\right)$, а $\mathbf{1}_{\xi}$ является наибольшим (т.е. самьм слабым) элементом $\mathbb{P}_{\xi}$, то фиксируем $\mathbb{P}_{\xi}$-терм $\overline{\mathbb{Q}}^{\xi}$

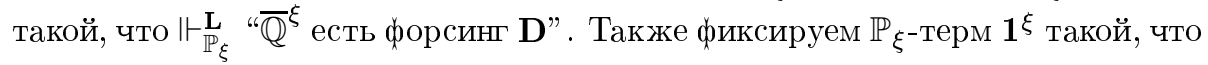
$\vdash_{\mathbb{P}_{\xi}}^{\mathbf{L}}$ “1 $\mathbf{1}^{\xi}$ есть пара $\langle\breve{\Lambda}, \breve{\mathbf{0}}\rangle$ ”. (Заметим, что пара $\langle\Lambda, \mathbf{0}\rangle$ - самьй слабьй элемент форсинга $\mathbf{D} ; \mathbf{0}(k)=0, \forall k$.)

$3^{\circ}$. Положим $Q^{\xi}=\left\{q: \exists p\left(\langle p, q\rangle \in \overline{\mathbb{Q}}^{\xi}\right)\right\}$. Из определений п. 4А следует, что $\overline{\mathbb{Q}}^{\xi}[G] \subseteq\left\{t[G]: t \in Q^{\xi}\right\}$ для любого $G$, так что требование $p(\xi) \in Q^{\xi}$ ниже в п. $4^{\circ}$ (введенное для того, чтобы $\mathbb{P}_{\xi+1}$ было множеством, а не собственным классом в $\mathfrak{M})$ не ограничивает общности.

$4^{\circ} . \mathbb{P}_{\xi+1}$ состоит из всех функций $p, \xi+1=\operatorname{dom} p$, таких, что $p\left\lceil\xi \in \mathbb{P}_{\xi}, p(\xi) \in Q^{\xi}\right.$ и $p \nmid \xi \Vdash_{\mathbb{P}_{\xi}}^{\mathbf{L}}$ “ $p(\xi) \in \overline{\mathbb{Q}}^{\xi " . ~}$

$5^{\circ}$. Порядок на $\mathbb{P}_{\xi+1}$ определяется так: $p \leqslant q$, когда $p\left\lceil\xi \leqslant q\left\lceil\xi\right.\right.$ и $p\left\lceil\xi \Vdash_{\mathbb{P}_{\xi}}^{\mathbf{L}}\right.$ “ $p(\xi) \leqslant$ $q(\xi)$ в смысле $\overline{\mathbb{Q}}^{\xi}$. Элемент $\mathbf{1}_{\xi+1} \in \mathbb{P}_{\xi+1}$ определяется следуюшим образом: $\mathbf{1}_{\xi+1}\left\lceil\xi=\mathbf{1}_{\xi}\right.$ и $\mathbf{1}_{\xi+1}(\xi)=\mathbf{1}^{\xi}$.

$6^{\circ}$. (Требование конечной поддержки форсинга.) Если $\vartheta \leqslant \omega_{2}^{\mathrm{L}}-$ предельньй ординал, то $\mathbb{P}_{\vartheta}$ состоит из всех функций $p, \vartheta=\operatorname{dom} p$, таких, что $p\left\lceil\xi \in \mathbb{P}_{\xi}\right.$ и $\|p\|=\left\{\xi<\vartheta: \neg p\left\lceil\xi \Vdash_{\mathbb{P}_{\xi}}^{\mathbf{L}} " p(\xi)=\mathbf{1}^{\xi "}\right\}-\right.$ конечное множество.

$7^{\circ}$. Порядок на $\mathbb{P}_{\vartheta}$ определяется так: $p \leqslant q$, когда $p\lceil\xi \leqslant q \uparrow \xi$ для всех $\xi<\vartheta$. Элемент $\mathbf{1}_{\vartheta} \in \mathbb{P}_{\vartheta}$ определяется так: $\mathbf{1}_{\vartheta}(\xi)=\mathbf{1}^{\xi}$ для всех $\xi<\vartheta$.

Форсинг $\mathbb{P}=\mathbb{P}_{\omega_{1}^{\mathbf{L}}} \in \mathbf{L}$ удовлетворяет УCA (в $\left.\mathbf{L}\right)$. В самом деле, итерации УСА форсингов с конечной поддержкой удовлетворяют УСA, a то, что $\mathbf{D}$ удовлетворяет УСА, следует из леммы 8.2 .

Фиксируем $\mathbb{P}$-генерическое над $\mathbf{L}$ множество $G \subseteq \mathbb{P}$. Каждое из множеств $G_{\xi}=$ $\left\{p\lceil\xi: p \in G\}\right.$ является $\mathbb{P}_{\xi}$-генерическим над $\mathbf{L}$.

Докажем, что в $\mathbf{L}[G]$ истинно $\mathrm{BP}\left(\boldsymbol{\Sigma}_{2}^{1}\right)$. Действительно, по теореме 5.3, достаточно проверить, что, для любого $\left.a \in \mathbf{L}[G] \cap \mathbb{N}^{\omega}, 1\right) \mathbf{C o h} \mathbf{L}[a] \cap \mathbf{L}[G] \neq \varnothing$ и 2) множество $\mathbf{L}[a] \cap \mathbb{N}^{\omega}$ является $\leqslant{ }^{*}$-ограниченным в $\mathbf{L}[G]$. Из УСА следует, при помощи обычных рассуждений, что $\omega_{1}^{\mathbf{L}[G]}=\omega_{1}^{\mathbf{L}}$, и потому $a \in \mathbf{L}\left[G_{\xi}\right] \cap \mathbb{N} \omega$ для некоторого $0<\xi<\omega_{1}^{\mathbf{L}}$. При этом, из обших свойств итерированного форсинга, $\mathbf{L}\left[G_{\xi+1}\right]$ является $(\mathbf{D})^{\mathbf{L}\left[G_{\xi}\right]}$-генерическим расширением $\mathbf{L}\left[G_{\xi}\right]$, так что 1$)$ следует из леммы 8.4, a 2$)$ простое свойство $\mathbf{D}$-генерических расширений (см. п. $8 \mathrm{~A})$.

Докажем, что в $\mathrm{L}[G]$ ложно $\operatorname{LM}\left(\boldsymbol{\Delta}_{2}^{1}\right)$. Согласно следствию $3.4(\mathrm{IV})$, достаточно доказать, что множество $\operatorname{Rand} \mathbf{L}[a]$ пусто в $\mathbf{L}[G]$ хотя бы для одного $a \in \mathbf{L}[G] \cap \mathbb{N} \omega$. Возьмем $a \in \mathbf{L} \cap \mathbb{N} \omega$, например, $a=\mathbf{0}$. Рассмотрим произвольное $x \in \mathbf{L}[G] \cap \mathbb{N} \omega$. Нужно доказать, что $x \notin \mathbf{R a n d} \mathbf{L}[a]$. Как и выше, $x \in \mathbf{L}\left[G_{\xi}\right]$ для некоторого $0<\xi<$ $\omega_{1}^{\mathbf{L}}$. Имеем $x=\mathbf{x}[G]$ для некоторого $\mathbb{P}_{\xi}$-имени $\mathbf{x} \in \mathbf{L}$.

Рассуждаем в $\mathbf{L}$. Множество $P \subseteq \mathbb{P}_{\xi}$ назовем иентрированным, если для любого конечного $P^{\prime} \subseteq P$ найдется "условие" $r \in \mathbb{P}_{\xi}$, удовлетворяюшее $r \leqslant p$ для каждого $p \in P$. Докажем, что форсинг $\mathbb{P}_{\xi}$ является $\sigma$-иентрированным, т.е. допускает представление в виде $\mathbb{P}_{\xi}=\bigcup_{n \in \mathbb{N}} P_{n}$, где все $P_{n}$ центрированы.

Рассуждаем индукцией по $\xi$. Для $\xi=0$ нечего доказьвать. Пусть ординал $\vartheta<\omega_{2}^{\mathrm{L}}$ 
пределен и для каждого $\xi<\vartheta$ мы имеем $\mathbb{P}_{\xi}=\bigcup_{n} P_{n}^{\xi}$, где все $P_{n}^{\xi}$ центрированы. Тогда $\mathbb{P}_{\vartheta}=\bigcup_{\xi<\vartheta} \bigcup_{n} P_{\xi n}$, где $P_{\xi n}=\left\{p \in \mathbb{P}_{\vartheta}:\|p\| \subseteq \xi \wedge p\left\lceil\xi \in P_{n}^{\xi}\right\}\right.$. Остается рассмотреть шаг $\xi \rightarrow \xi+1$. Предположим, что $\mathbb{P}_{\xi}=\bigcup_{n} P_{n}$, где все $P_{n}$ центрированы. Для $n \in \mathbb{N}$ и $s \in \mathbb{N}<\omega$ определим

$$
P_{n s}=\left\{p \in \mathbb{P}_{\xi+1}: \exists q \in P_{n}\left(q \leqslant p\left\lceil\xi \wedge q \Vdash_{\mathbb{P}_{\xi}}^{\mathbf{L}} " s_{p(\xi)}=\breve{s} "\right)\right\},\right.
$$

где для каждого “условия" $p=\langle s, f\rangle \in \mathbf{D}$ через $s_{p}$ обозначается $s$. Легко видеть, что каждое множество $P_{n s}$ центрировано и $\mathbb{P}_{\xi+1}=\bigcup_{s \in \mathbb{N}<\omega} \bigcup_{n} P_{s n}$, что завершает доказательство $\sigma$-центрированности.

Итак, мы имеем (все еще в $\mathbf{L})$ представление $\mathbb{P}_{\xi}=\bigcup_{n} P_{n}$, где все множества $P_{n}$ центрированы. Через $\mathscr{T}_{n}$ обозначим множество всех деревьев $T \subseteq 2^{<\omega}$, без $\subseteq$-макси-

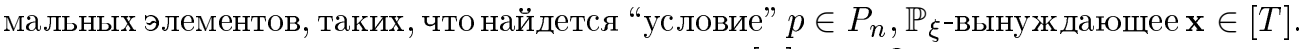
Из центрированности $P_{n}$ следует, что множества $[T], T \in \mathscr{T}_{n}$, образуют центрированную систему (замкнутых подмножеств $2^{\omega}$ ), так что найдется точка $x_{n} \in \bigcap_{T \in \mathscr{T}_{n}}[T]$. Можно подобрать последовательность деревьев $T_{k} \subseteq 2^{<\omega}$, так что множества $\left[T_{k}\right]$ попарно дизъюнктны, не пересекают $X=\left\{x_{n}: n \in \mathbb{N}\right\}$ и $\sum_{k} \lambda\left(\left[T_{k}\right]\right)=1$.

Теперь, уже в $\mathbf{L}[G]$, мы утверждаем, что $x$ не принадлежит $\mathbf{F}_{\sigma}$-множеству $\bigcup_{k}\left[T_{k}\right]$, следовательно, еㅡинадлежит Rand $\mathbf{L}[a]$, так как дополнение $\bigcup_{k}\left[T_{k}\right]$ имеет $\boldsymbol{\lambda}$-меру 0 . Пусть, напротив, $x$ принадлежит некоторому $\left[T_{k}\right]$. Это вынуждается каким-нибудь условием $p \in G$. Тогда, для некоторого $n$, мы имеем $p \in P_{n}$, и, соответственно, $T_{k} \in \mathscr{T}_{n}$, откуда по построению вытекает $x_{n} \in\left[T_{k}\right]$ - а это противоречит выбору деревьев $T_{k}$.

(теорема 8.1(3))

8Г. $\left(\mathrm{BP}\left(\boldsymbol{\Delta}_{2}^{1}\right) \wedge \mathrm{LM}\left(\boldsymbol{\Delta}_{2}^{1}\right)\right) \not \Rightarrow \mathrm{BP}\left(\boldsymbol{\Sigma}_{2}^{1}\right)$. Искомая модель получается одновременной итерацией форсинга Коэна $\mathbf{C}$ и случайного форсинга $\mathbf{B}$, длины $\omega_{1}^{\mathbf{L}}$ и с конечной поддержкой. Именно, на четных шагах (а четньми считаются ординалы вида: предельньй ординал + четное натуральное число, включая 0) действует $\mathbf{C}$, а на нечетных - $\mathbf{B}$. (Можно определить и наоборот.)

Точнее говоря, итерированньй форсинг $\mathbb{P}$ строится по схеме $1^{\circ}-7^{\circ}$ из п. $8 \mathrm{~B}$, со следующей поправкой.

$2^{\circ}$. Если форсинг $\mathbb{P}_{\xi}$ определен (и состоит из функций $p$ с $\xi=\operatorname{dom} p$ ), a $\mathbf{1}_{\xi}-$ наибольший (т.е. самьй слабый) элемент $\mathbb{P}_{\xi}$, то фиксируем $\mathbb{P}_{\xi}$-терм $\overline{\mathbb{Q}}^{\xi}$ такой, что

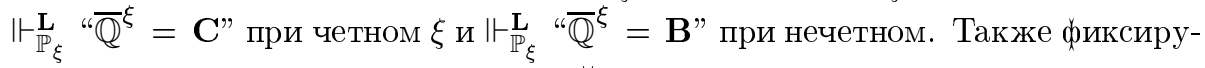
ем $\mathbb{P}_{\xi^{-т е р м ~}} \mathbf{1}^{\xi}$ такой, что $\Vdash_{\mathbb{P}_{\xi}}^{\mathbf{L}}$ “1 $\mathbf{1}^{\xi}=\breve{\Lambda}$ ” при четном $\xi$ и $\Vdash_{\mathbb{P}_{\xi}}^{\mathbf{L}}$ " $\mathbf{1}^{\xi}={ }^{u} 2^{<\omega}$ " при нечетном. (Заметим, что $\Lambda$ - самый слабый элемент $\mathbf{C}$, а $2^{<\omega}$ - самый слабый элемент В.)

Фиксируем $\mathbb{P}$-генерическое над $\mathbf{L}$ множество $G \subseteq \mathbb{P}$. Каждое из множеств $G_{\xi}=\left\{p\lceil\xi: p \in G\}\right.$ является $\mathbb{P}_{\xi}$-генерическим над $\mathbf{L}$. Более того, мы имеем $\mathbf{L}[G]=\mathbf{L}\left[\left\{a_{\xi}\right\}_{\xi<\omega_{1}^{\mathbf{L}}}\right]$, где $a_{\xi} \in \mathbf{C o h} \mathbf{L}\left[\left\{a_{\eta}\right\}_{\eta<\xi}\right]$ при четных $\xi$ и, соответственно, $a_{\xi} \in \operatorname{Rand} \mathbf{L}\left[\left\{a_{\eta}\right\}_{\eta<\xi}\right]$ при нечетных.

Наша цель - показать, что в такой модели $\mathbf{L}[G]$ истинны $\operatorname{BP}\left(\boldsymbol{\Delta}_{2}^{1}\right)$ и $\operatorname{LM}\left(\boldsymbol{\Delta}_{2}^{1}\right)$, но ложно $\mathrm{BP}\left(\boldsymbol{\Sigma}_{2}^{1}\right)$. "Положительная" часть этого утверждения легко сводится к уже полученным вьше результатам. Именно, поскольку С и $\mathbf{B}$ - УСА-форсинги, наш итерированный форсинг также удовлетворяет УСА, а потому $\omega_{1}^{\mathbf{L}[G]}=\omega_{1}^{\mathbf{L}}$. Тем самым, для 
каждого $a \in \mathbf{L}[G] \cap \mathbb{N}^{\omega}$ найдется ординал $\xi<\omega_{1}^{\mathbf{L}}$ такой, что $a \in \mathbf{L}\left[G_{\xi}\right]$. Отсюда следует $\mathbf{C o h} \mathbf{L}[a] \neq \varnothing$ и Rand $\mathbf{L}[a] \neq \varnothing$. Мы получаем $\operatorname{LM}\left(\boldsymbol{\Delta}_{2}^{1}\right)$ и $\operatorname{BP}\left(\boldsymbol{\Delta}_{2}^{1}\right)$ (следствие 3.4).

Что же касается “отрицательной” части, для вывода $\neg \mathrm{BP}\left(\boldsymbol{\Sigma}_{2}^{1}\right)$ достаточно, согласно теореме 5.3, проверить, что хотя бы одно из множеств вида $\mathbf{L}[a] \cap \mathbb{N}^{\omega}, a \in \mathbb{N}^{\omega}$, не является ограниченным в $\mathbf{L}[G]$. Мы собираемся доказать, что уже $\mathbf{L} \cap \mathbb{N}^{\omega}$ не является ограниченньм в $\mathbf{L}[G]$. Доказательство этого предложения основано на проверке сохранения одного свойства форсинга в рассматриваемой итерации.

ОПРЕДЕЛЕНИЕ 8.5. Форсинг $\mathscr{P}$ назьвается $\leqslant{ }^{*}$-хорошим, если для всякого $\mathscr{P}$-генерического над универсумом $\mathbf{V}$ множества $G \subseteq \mathscr{P}$ и для всякого $f \in \mathbf{V}[G] \cap \mathbb{N}^{\omega}$ найдется $g \in \mathbf{V} \cap \mathbb{N}^{\omega}$ такое, что $\forall x \in \mathbf{V} \cap \mathbb{N}^{\omega}\left(x \leqslant{ }^{*} f \Longrightarrow x \leqslant^{*} g\right)$.

ТеОрема 8.6. В $\mathbf{L}$ истинно, что кажддй форсинг $\mathbb{P}_{\xi}, \xi<\omega_{2}^{\mathbf{L}}$, является $\leqslant^{*}-$-хорошим.

Отсюда следует, что множество $\mathbf{L} \cap \mathbb{N}^{\omega}$ не являетс $\leqslant$-ограниченньм ни в каком $\mathbb{P}_{\xi}$-генерическом расширении $\mathbf{L}$, в частности, ни в одном из классов $\mathbf{L}\left[G_{\xi}\right]$. Однако, как мы уже отмечали, любое $x \in \mathbf{L}[G] \cap \mathbb{N}^{\omega}$ принадлежит $\mathbf{L}\left[G_{\xi}\right] \cap \mathbb{N}^{\omega}$ для подходящего $\xi<\omega_{2}^{\mathbf{L}}$, так что $\mathbf{L} \cap \mathbb{N}^{\omega}$ не является $\leqslant *$-ограниченньм даже в $\mathbf{L}[G]$, что и требовалось. Итак, задача сводится к доказательству теоремы 8.6.

Мы предварим его двумя леммами.

Лемма 8.7. Форсинги $\mathbf{B} и \mathbf{C}-\leqslant^{*}$-хорошие.

ДокАЗАТЕльство. Начнем с форсинга В. Пусть множество $G \subseteq \mathbf{B}$ является генерическим над $\mathbf{V}$ и $f \in \mathbb{N}^{\omega} \cap \mathbf{V}[G]$. Имеем $f=\underline{f}[G]$, где $\underline{f} \in \mathbf{V}$. Можно предполагать, что $\Vdash_{\mathbf{B}} \underline{f} \in \mathbb{N}^{\omega}$. Докажем, что множество

$$
D^{\prime}=\left\{T^{\prime} \in \mathbf{B}: \exists g \in \mathbb{N}^{\omega}\left(T^{\prime} \Vdash_{\mathbf{B}} \underline{f} \leqslant{ }^{*} \breve{g}\right)\right\}
$$

плотно в В. (Отсюда, благодаря генеричности $G$, следует, что $f \leqslant{ }^{*} g$ для некоторой $g \in \mathbf{V} \cap \mathbb{N}^{\omega}$, и “хорошесть" очевидна.) Пусть $T_{0} \in \mathbf{B}$. Множество

$$
D_{n}=\left\{T \in \mathbf{B}: T \subseteq T_{0} \wedge \exists k\left(T \Vdash_{\mathbf{B}} \underline{f}(\breve{n})=\breve{k}\right)\right\}
$$

плотно в $\mathbf{B}$ под $T_{0}$ при любом $n$. Выбрав максимальную антицепь $A_{n} \subseteq D_{n}$ (она счетна благодаря УСА), мы можем подобрать конечное множество $A_{n}^{\prime} \subseteq A_{n}$ такое, что множество $X_{n}=\bigcup_{T \in A_{n}^{\prime}}[T]$ (замкнутое подмножество $\left.\left[T_{0}\right]\right)$ удовлетворяет $\boldsymbol{\lambda}\left(X_{n}\right) \geqslant$ $\alpha\left(1-2^{-n-2}\right)$, где $\alpha=\lambda\left(\left[T_{0}\right]\right)$. Тогда $T_{n}=\bigcup A_{n}^{\prime}-$ дерево в $2^{<\omega}$ (без максимальных элементов) и $\left[T_{n}\right]=X_{n}$, так что $T_{n} \in \mathbf{B}$. А в силу конечности $A_{n}^{\prime}$ найдется $k_{n}$ такое, что $T_{n} \Vdash_{\mathbf{B}} \underline{f}(\breve{n}) \leqslant \breve{k}_{n}$. Наконец, положим $T^{\prime}=\bigcap_{n} T_{n}$. По построению, $\boldsymbol{\lambda}\left(\left[T^{\prime}\right]\right) \geqslant \frac{\alpha}{2}$, так что $T^{\prime} \in \mathbf{B}$. Кроме того, $T^{\prime} \Vdash_{\mathbf{B}} \underline{f}(\breve{n}) \leqslant \breve{k}_{n}$ для всех $n$, т.е. $T^{\prime} \Vdash_{\mathbf{B}} \underline{f} \leqslant{ }^{*} \breve{g}$, где $g(n)=k_{n}, \forall n$, так что $T^{\prime} \in D^{\prime}$.

Для форсинга $\mathbf{C}=\mathbb{N}<\omega$ рассуждаем иначе. Положим

$$
F_{n s}=\left\{x \in \mathbb{N}^{\omega}: s \Vdash_{\mathbf{C}} \breve{x} \leqslant{ }_{n} \underline{f}\right\} \quad \text { для } s \in \mathbf{C}=\mathbb{N}^{<\omega} \text { и } n \in \mathbb{N},
$$

где $x \leqslant n y$ означает, что $x(i) \leqslant y(i)$ для всех $i \geqslant n$. Понятно, что $M_{n s i}=$ $\max _{h \in F_{n s}} h(i)$ конечно при $i \geqslant n$. Положим $f_{n s}(i)=M_{n s i}$ при $i \geqslant n$ и $=0$ при $i<n$. Таким образом, $x \leqslant n f_{n s}$ для всех $x \in F_{n s}$. Остается взять $g \in \mathbb{N}^{\omega}$ так, чтобы $f_{n s} \leqslant{ }^{*} g$ для всех $n, s$.

Следуюшая лемма характеризует $\leqslant *$-хорошие форсинги в терминах отношения вынуждения. Напомним, что $\Vdash \mathscr{P}$ означает $\Vdash \stackrel{\mathrm{V}}{\mathrm{P}}$. 
Лемма 8.8. УСА-форсинг Я является $\leqslant{ }^{*}$-хорошим в том и только том случае, когда для всякого имени $t$ такого, что $\Vdash \mathscr{P} t \in \mathbb{N} \omega$, найдется функиия $g \in \mathbb{N}^{\omega}$, удовлетворяющая

$$
\forall x \in \mathbb{N}^{\omega}\left(\left(\exists p \in \mathscr{P}\left(p \Vdash \mathscr{P} \breve{x} \leqslant \leqslant^{*} t\right)\right) \Longrightarrow x \leqslant^{*} g\right) .
$$

ДокАЗАТЕЛЬСТво. В нетривиальном направлении, пусть $\mathscr{P}$ - хороший форсинг и $\Vdash \mathscr{P} t \in \mathbb{N}^{\omega}$. Тогда множество

$$
D=\left\{p \in \mathscr{P}: \exists g=g_{p} \in \mathbb{N}^{\omega} \forall x \in \mathbb{N}^{\omega}\left(p \Vdash \mathscr{P}\left(\breve{x} \leqslant^{*} t \Longrightarrow \breve{x} \leqslant{ }^{*} \breve{g}\right)\right)\right\}
$$

плотно в $\mathscr{P}$. Согласно УСА, найдется счетная максимальная антицеп $A \subseteq D$. Вследствие счетности, найдется функция $g \in \mathbb{N}^{\omega}$ такая, что $g_{p} \leqslant{ }^{*} g$ для всех $p \in A$. Нетрудно проверить, что $g$ обеспечивает “хорошесть" $\mathscr{P}$.

ДокАЗАТЕЛЬСТво теОремы 8.6. “Хорошесть $\mathbb{P}_{\xi}$ в $\mathbf{L}$ доказывается индукцией по $\xi<\omega_{2}^{\mathrm{L}}$. Лемма 8.7 обосновьвает индуктивный шаг $\xi \rightarrow \xi+1$, поскольку, в рассматриваемой итерации, $\mathbf{L}\left[G_{\xi+1}\right]$ есть $\mathbf{B}$-генерическое или $\mathbf{C}$-генерическое расширение $\mathbf{L}\left[G_{\xi}\right]$. Для вьполнения предельного шага, рассмотрим предельный ординал $\xi<\omega_{2}^{\mathbf{L}}$. Тогда найдется строго возрастающая последовательность $\left\{\xi_{n}\right\}_{n \in \mathbb{N}} \in \mathbf{L}$ меньших ординалов с $\xi=\sup _{n} \xi_{n}$. Фиксируем $f \in \mathbf{L}[G] \cap \mathbb{N}^{\omega}$. Найдется имя $\underline{f} \in \mathbf{L}$ такое, что $f=\underline{f}[G]$. Можно предполагать, что $\Vdash_{\mathbb{P}}^{\mathbf{L}}$ " $f \in \mathbb{N}^{\omega}$ ".

Рассмотрим любое $n$. Рассуждая в $\mathbf{L}\left[G_{\xi_{n}}\right]$, мы можем построить последовательность $\left\{p_{n}^{k}\right\}_{k \in \mathbb{N}} \in \mathbf{L}\left[G_{\xi_{n}}\right]$ "условий” $p_{n}^{k} \in \mathbb{P}$ так, что при любом $k$ вьполнено $p_{n}^{k+1} \leqslant p_{n}^{k}$ (т.е. $p_{n}^{k+1}$ сильнее в $\left.\mathbb{P}\right), p_{n}^{k}\left\lceil\xi_{n} \in G_{\xi_{n}}\right.$ и $p_{n}^{k} \Vdash_{\mathbb{P}}^{\mathbf{L}}$ “ $\underline{f}\left\lceil\breve{k}={ }^{u} s_{k}\right.$ ” для некоторого $s_{k} \in \mathbb{N}<\omega$. Тогда $s_{k} \subset s_{k+1}$, поэтому $f_{n}=\bigcup_{k} s_{k} \in \mathbf{L}\left[G_{\xi_{n}}^{-}\right] \cap \mathbb{N}^{\omega}$. Найдется имя $\underline{f_{n}} \in \mathbf{L}$ такое, что $f_{n}=\underline{f_{n}}\left[G_{\xi_{n}}\right]$ и $\Vdash_{\mathbb{P}_{\xi_{n}}}^{\mathrm{L}} \quad \underline{f_{n}} \in \mathbb{N} \omega$ ”. Это построение можно организовать так, чтобы последовательность имен $\left\{\underline{f}_{n}\right\}_{n \in \mathbb{N}}$ принадлежала $\mathbf{L}$. Вследствие “хорошести" $\mathbb{P}_{\xi_{n}}$ и леммы 8.8, для каждого $n$ найдется функция $g_{n} \in \mathbf{L} \cap \mathbb{N}^{\omega}$ такая, что

$$
\forall x \in \mathbf{L} \cap \mathbb{N}^{\omega}\left(\left(\exists p \in \mathbb{P}_{\xi_{n}}\left(p \Vdash_{\mathbb{P}_{\xi_{n}}}^{\mathbf{L}} \breve{x} \leqslant{ }^{*} \underline{f_{n}}\right)\right) \Longrightarrow x \leqslant{ }^{*} g_{n}\right) .
$$

Снова можно считать, что $\left\{g_{n}\right\}_{n \in \mathbb{N}} \in \mathbf{L}$. В этом предположении найдется $g \in \mathbf{L} \cap \mathbb{N} \omega$ такая, что $g_{n} \leqslant^{*} g, \forall n$. Мы утверждаем, что $g$ доказывает “хорошесть" $\mathbb{P}_{\xi}$ по отношению к $f$, т.е. $x \leqslant{ }^{*} f \Longrightarrow x \leqslant{ }^{*} g$ для любого $x \in \mathbf{L} \cap \mathbb{N}^{\omega}$.

Предположим противное: $x \in \mathbf{L} \cap \mathbb{N}^{\omega}, x \leqslant^{*} f$, но $x \mathbb{Z}^{*} g$. Тогда найдутся $m$ и “условие" $p \in G$ такое, что $p \Vdash_{\mathbb{P}}^{\mathbf{L}} \breve{x} \leqslant_{m}^{*} f$, где $x \leqslant_{m}^{*} y$ означает, что $x(k) \leqslant y(k)$ для всех $k \geqslant m$. Имеем $\|p\| \subseteq \xi_{n}$ для некоторого $n$, поскольку наша итерация - с конечной поддержкой. В наших предположениях вьполнено $x \mathbb{*}^{*} g_{n}$, в частности, $x \mathbb{z}_{m}^{*} g_{n}$, другими словами, найдется $k \geqslant m$ такое, что $j=g_{n}(k)<x(k)$. По определению $g_{n}$, это означает, что $p_{n}^{k} \Vdash_{\mathbb{P}}^{\mathbf{L}}$ “ $\underline{f}(\breve{k})=\breve{j}$ ”.

Напомним, что по построению $p_{n}^{k} \uparrow \xi_{n} \in G_{\xi_{n}}$. Отсюда нетрудно вывести существование $\mathbb{P}$-генерического над $\mathbf{L}$ множества $G^{\prime} \subseteq \mathbb{P}$ такого, что $G_{\xi_{n}}^{\prime}=G_{\xi_{n}}$ и $p_{n}^{k} \in G^{\prime}$. Тогда $f^{\prime}=f\left[G^{\prime}\right]$ удовлетворяет $f^{\prime}(k)=j$. С другой стороны, “условие” $p$ принадлежит $G^{\prime}$, поскольку $\|p\| \subseteq \xi_{n}$ и $G_{\xi_{n}}^{\prime}=G_{\xi_{n}}$. Отсюда вытекает $x \leqslant_{m}^{*} f^{\prime}$ по выбору $p$, в частности, $x(k) \leqslant f^{\prime}(k)$, так как $k \geqslant m$. Однако $x(k)>j$, противоречие. 
8Д. $\left(\operatorname{BP}\left(\Sigma_{2}^{1}\right) \wedge \operatorname{LM}\left(\boldsymbol{\Delta}_{2}^{1}\right)\right) \not \operatorname{LM}\left(\Sigma_{2}^{1}\right)$. Модель для доказательства невыводимости этой импликации получается итерацией доминирующего форсинга $\mathbf{D}$ и случайного форсинга $\mathbf{B}$, длины $\omega_{1}^{\mathrm{L}}$ и с конечной поддержой. Итак, рассматривается итерированньй форсинг $\mathbb{P}$, построенньй по схеме $1^{\circ}-7^{\circ}$ из п. $8 \mathrm{~B}$, со следуюшей поправкой.

$2^{\circ}$. Если форсинг $\mathbb{P}_{\xi}$ определен (и состоит из функций $p$ c $\xi=\operatorname{dom} p$ ), а $\mathbf{1}_{\xi}$ - наибольший (т.е. самый слабый) элемент $\mathbb{P}_{\xi}$, то фиксируем $\mathbb{P}_{\xi}$-терм $\overline{\mathbb{Q}}^{\xi}$ такой, что

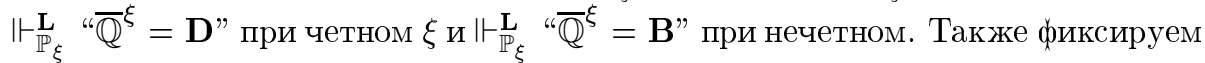

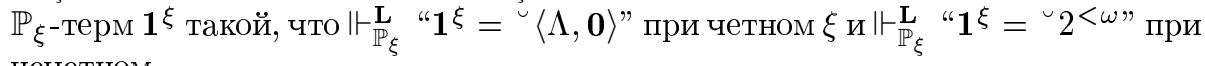
нечетном.

Фиксируем $\mathbb{P}$-генерическое над $\mathbf{L}$ множество $G \subseteq \mathbb{P}$. Каждое из множеств $G_{\xi}=$

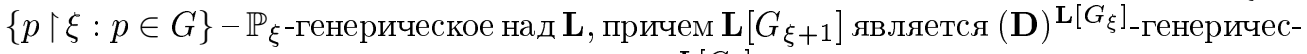

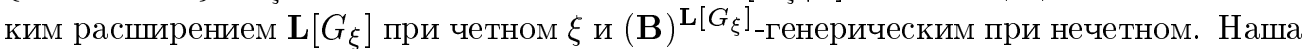
цель - показать, что в $\mathbf{L}[G]$ истинны $\mathrm{BP}\left(\boldsymbol{\Sigma}_{2}^{1}\right)$ и $\operatorname{LM}\left(\boldsymbol{\Delta}_{2}^{1}\right)$, но ложно $\operatorname{LM}\left(\boldsymbol{\Sigma}_{2}^{1}\right)$. "Положительная" часть этого утверждения аналогична некоторым уже полученньм вьше результатам. Именно, ВР $\left(\boldsymbol{\Sigma}_{2}^{1}\right)$ вьполняется по тем же причинам, что и в модели, построенной в п. $8 \mathrm{~B}$, a $\operatorname{LM}\left(\boldsymbol{\Delta}_{2}^{1}\right)$ - по тем же причинам, что и в модели, построенной в п. $8 \Gamma$.

Что же касается "отрицательной” части, для вьвода $\neg \operatorname{LM}\left(\Sigma_{2}^{1}\right)$ в $\mathbf{L}[G]$ мы собираемся воспользоватьс теоремой 5.3. Достаточно проверить, что множество $\boldsymbol{\ell}_{1} \cap \mathbf{L}$ не является $\leqslant^{*}$-ограниченньм в $\boldsymbol{\ell}_{1} \cap \mathbf{L}[G]$, т.е. нет такой функции $f \in \boldsymbol{\ell}_{1} \cap \mathbf{L}[G]$, что $g \leqslant * f$ всякий раз, когда $g \in \boldsymbol{\ell}_{1} \cap \mathbf{L}$. Доказательство этого предложения основано на проверке сохранения одного свойства форсинга в рассматриваемой итерации.

Следующее определение является вариантом определения 8.5.

ОПРЕДЕЛЕНИЕ 8.9. Форсинг $\mathscr{P}$ назьвается $\ell_{1}$-хорошим, если для любого $\mathscr{P}$-генерического над универсумом $\mathbf{V}$ множества $G \subseteq \mathscr{P}$ и любого $f \in \mathbf{V}[G] \cap \boldsymbol{\ell}_{1}$ найдется $g \in \mathbf{V} \cap \boldsymbol{\ell}_{1}$ такое, что $\forall x \in \mathbf{V} \cap \boldsymbol{\ell}_{1}\left(x \leqslant * f \Longrightarrow x \leqslant^{*} g\right)$.

Лемма 8.10. Форсинги $\mathbf{B}$ и $\mathbf{D}$ являются $\ell_{1}$-хорошими.

ДокАЗАТЕльство. Доказательство для форсинга $\mathbf{B}$ отличается от соответствующей части доказательства леммы 8.7. Пусть множество $G \subseteq \mathbf{B}$ является генерическим над $\mathbf{V}$ и $f \in \boldsymbol{\ell}_{1} \cap \mathbf{V}[G]$. По определению, сумма $\sum_{n} f(n)$ конечна, следовательно, ее можно сделать сколь угодно малой, изменив лишь конечное число значений $f$. Поскольку эта операция не влияет на отношение $\leqslant^{*}$, можно предполагать, что $\sum_{n} f(n)<1$.

Имеем $f=f[G]$, где $f \in \mathbf{V}$. В силу сказанного вьше, можно предполагать, что $\Vdash_{\text {В }} \sum_{n} \underline{f}(n)<\overline{1}$. Используя УСА, нетрудно проверить, что для всякой пары $n, i \in \mathbb{N}$ сушествует борелевское множество $B_{n i} \subseteq \mathbb{N}^{\omega}$ такое, что

$$
\forall T \in \mathbf{B}\left(\left(T \Vdash_{\mathbf{B}} \underline{f}(n) \geqslant i 2^{-n}\right) \Longleftrightarrow \boldsymbol{\lambda}\left([T] \backslash B_{n i}\right)=0\right) .
$$

Положим $g_{m}(n)=\sup \left\{i: \boldsymbol{\lambda}\left(B_{n i}\right)>\frac{1}{m+1}\right\}$ и докажем

$$
\sum_{n} g_{m}(n) 2^{-n} \leqslant m+1, \quad \forall m .
$$

Предположим противное: тогда $\sum_{n<n_{0}} g_{m}(n) 2^{-n}>m+1$ для каких-то $m$ и $n_{0}$. Положим $U=\left\{\langle n, i, j\rangle: n<n_{0} \wedge i<g_{m}(n) \wedge j<2^{n_{0}-n}\right\}$ и $B_{n i j}=B_{n i}$ для $\langle n, i, j\rangle \in U$. 
По определению $g_{m}(n)$, мы имеем $\boldsymbol{\lambda}\left(B_{n i j}\right)>\frac{1}{m+1}$ при $\langle n, i, j\rangle \in U$, откуда, по выбору $m_{0}, n$, следует

$$
\sum_{\langle n, i, j\rangle \in U} \lambda\left(B_{n i j}\right)>\frac{1}{m+1} \sum_{n<n_{0}} g_{m}(n) 2^{n_{0}-n}>2^{n_{0}}
$$

Следовательно, существует множество $V \subseteq U$, содержащее $2^{n_{0}}+1$ элементов и такое, что пересечение $B=\bigcap_{\langle n, i, j\rangle \in V} B_{n i j}$ удовлетворяет $\boldsymbol{\lambda}(B)>0 .{ }^{33}$ Положим

$$
\begin{aligned}
V_{n} & =\{\langle i, j\rangle:\langle n, i, j\rangle \in V\}, & & N=\left\{n<n_{0}: V_{n} \neq \varnothing\right\}, \\
I_{n} & =\left\{i: \exists j\left(\langle i, j\rangle \in V_{n}\right)\right\}, & & i_{n}=\sup I_{n} \quad(\text { при } n \in N) .
\end{aligned}
$$

Раз $\boldsymbol{\lambda}(B)>0$, найдется "условие" $T \in \mathbf{B}$ c $[T] \subseteq B$. Из (1) следует, что $T \Vdash_{\mathbf{B}}$ " $f(n) \geqslant i_{n} 2^{-n}$ " для каждого $n \in N$. (Для уменьшения громоздкости значки “ над $i_{n}$ и т. п. опускаются.) Однако, по определению $U$, мы имеем $i_{n} 2^{n_{0}-n} \geqslant \# V_{n}$, откуда следует $T \Vdash_{\mathbf{B}}$ “ $\underline{f}(n) \geqslant 2^{-n_{0}} \# V_{n}$ ” (при $\left.n \in N\right)$. Тем самым, $T \Vdash_{\mathbf{B}}$ " $\sum_{n<n_{0}} \underline{f}(n) \geqslant$ $2^{-n_{0}} \sum_{n \in N} \# V_{n}=2^{-n_{0}} \# V>1$ ", что противоречит нашему предположению $\Vdash_{\mathbf{B}}$ " $\sum_{n} \underline{f}(n)<1 "$. Это завершает доказательство (2).

Положим $h_{m}(n)=g_{m}(n) 2^{-n}$. Таким образом, $\sum_{n} h_{m}(n)<\infty$, откуда следует, что найдется функция $g \in \ell_{1}$ такая, что $h_{m} \leqslant{ }^{*} g, \forall m$. Покажем, что $g$ влечет "хорошесть" В по отношению к $f$. Пусть $h \in \ell_{1} \cap \mathbf{V}$ и $h \leqslant *$. Найдется “условие" $T \in G$ такое, что $T \Vdash_{\mathbf{B}} \forall n \geqslant k(h(n) \leqslant \underline{f}(n))$ для некоторого $k$. Возьмем $m$, достаточно

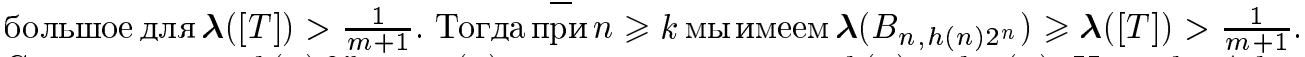
Следовательно, $h(n) 2^{n} \leqslant g_{m}(n)$, или, что то же самое, $h(n) \leqslant h_{m}(n)$. Итак, $h \leqslant{ }^{*} h_{n}$, откуда $h \leqslant{ }^{*} g$, что и требовалось.

Доказательство для форсинга $\mathbf{D}$ состоит в следуюшем. Во-первых, нетрудно проверить, что ЧУ множество $\mathbf{D}$ являетс отделимыл: это означает, что для любой пары “условий” $p \not q$ из $\mathbf{D}$ найдется "условие" $r \leqslant p, r \notin q$. В этом случае найдется (см. [29; теорема 29B]) полная булева алгебра $\mathscr{B}$ такая, что $\mathbf{D}$ порядково изоморфно некоторому плотному подмножеству множества $\mathscr{P}=\mathscr{B} \backslash\left\{0_{\mathscr{B}}\right\}$ всех ненулевых элементов $\mathscr{B}$ с порядком $a \leqslant b$, когда $a \cdot b=b$. Таким образом, достаточно доказать, что $\mathscr{P}$ является $\Delta$-хорошим. Заметим, что множество $\mathbf{D} \sigma$-центрировано: $\mathbf{D}=\bigcup_{s \in \mathbb{N}<\omega} D_{s}$, где каждое $D_{s}=\left\{\langle s, f\rangle: f \in \mathbb{N}^{\omega}\right\}$ центрировано. Таким образом, и $\mathscr{P} \sigma$-центрировано. Пусть $\mathscr{P}=\bigcup_{n} P_{n}$, где каждое $P_{n}$ центрировано. Можно предполагать, что каждое $P_{n}$ является максимальным центрированньм множеством - замкнутым относительно · (умножения в булевой алгебре $\mathscr{B}$ ), удовлетворяющим $a \geqslant b \in P_{n} \Longrightarrow a \in P_{n}$ и обязательно содержащим один из любых двух взаимно дополнительных элементов

\footnotetext{
${ }^{33}$ Здесь мы ссылаемся на следующую лемму из теории меры: если $M \leqslant K$ u семейство $\left\{X_{k}: k<K\right\}$ измеримых множеств удовлетворяет $\boldsymbol{\lambda}\left(\bigcap_{k \in V} X_{k}\right)>0$ для любого $V \subseteq K c$ card $V=M$, mo $\sum_{k<K} \boldsymbol{\lambda}\left(X_{k}\right) \leqslant M-1$. Доказательство индукцией по $M$ одновременно для всех $K \geqslant M$ основано на разложении (где $\overline{X_{K-1}}-$ дополнение $X_{K-1}$ )
}

$$
\begin{aligned}
\sum_{k<K} \boldsymbol{\lambda}\left(X_{k}\right) & =\boldsymbol{\lambda}\left(X_{K-1}\right)+\sum_{k<K-1} \boldsymbol{\lambda}\left(X_{k} \cap X_{K-1}\right)+\sum_{k<K-1} \boldsymbol{\lambda}\left(X_{k} \cap \overline{X_{K-1}}\right) \\
& \leqslant \boldsymbol{\lambda}\left(X_{K-1}\right)+(M-2) \boldsymbol{\lambda}\left(X_{K-1}\right)+(M-1) \boldsymbol{\lambda}\left(\overline{X_{K-1}}\right)=M-1 .
\end{aligned}
$$


$a,-a$. В этом случае существует конечно-аддитивная строго положительная на $\mathscr{P}$ мера $\mu(a)=\sum_{a \in P_{n}} 2^{-n}$ на $\mathscr{B}$. Имея такую меру, мы можем повторить для $\mathscr{P}$ доказательство, данное для $\mathbf{B}$.

Отсюда, совершенно аналогично теореме 8.6, получаем следующее утверждение.

ТеОрема 8.11. В L истинно, что каждый форсинг $\mathbb{P}_{\xi}, \xi<\omega_{2}^{\mathbf{L}}$, является $\ell_{1}$-хорошим.

В свою очередь, из этой теоремы следует, что множество $\mathbf{L} \cap \boldsymbol{\ell}_{1}$ не является $\leqslant$ *-ограниченным в $\boldsymbol{\ell}_{1}$ ни в каком $\mathbb{P}_{\xi}$-генерическом расширении $\mathbf{L}$, в частности, ни в одном из классов $\mathbf{L}\left[G_{\xi}\right]$. Однако, вследствие УСА, любое $x \in \mathbf{L}[G] \cap \mathbb{N}^{\omega}$ принадлежит $\mathbf{L}\left[G_{\xi}\right] \cap$ $\mathbb{N}^{\omega}$ для подходящего $\xi<\omega_{2}^{\mathbf{L}}$, так что $\mathbf{L} \cap \mathbb{N}^{\omega}$ не является $\leqslant^{*}$-ограниченным в $\boldsymbol{\ell}_{1}$ даже в $\mathbf{L}[G]$, что и требовалось.

(теорема $8.1(2))$

8E. $\operatorname{LM}\left(\boldsymbol{\Delta}_{2}^{1}\right) \not \mathrm{BP}\left(\boldsymbol{\Delta}_{2}^{1}\right)$. Здесь устанавливается невыводимость импликации (5) теоремы 8.1. На самом деле, мы докажем даже, что $\operatorname{LM}\left(\boldsymbol{\Delta}_{2}^{1}\right) \neq \operatorname{BP}\left(\boldsymbol{\Delta}_{2}^{1}\right) \vee \operatorname{LM}\left(\boldsymbol{\Sigma}_{2}^{1}\right)$, откуда, в частности, следует, что $\operatorname{LM}\left(\boldsymbol{\Delta}_{2}^{1}\right) \not \operatorname{LM}\left(\boldsymbol{\Sigma}_{2}^{1}\right)$. Это, конечно, слабее, чем (2) теоремы 8.1, но зато проще доказьвается. Искомая генерическая модель может быть охарактеризована как итерированное, длины $\omega_{1}^{\mathbf{L}}$ и со счетной ${ }^{34}$ поддержкой, генерическое расширение $\mathbf{L}$ посредством случайного форсинга $\mathbf{B}$. K счастью, эта сложная конструкция допускает, в данном случае, простую геометрическую форму.

Ниже рассматриваются пространства вида $\mathscr{C}^{\vartheta}, \vartheta<\omega_{1}^{\mathbf{L}}$, где, напомним, $\mathscr{C}=2^{\omega}$. Мы используем полужирные буквы вроде $\boldsymbol{x}, \boldsymbol{y}$ для обозначения точек этих пространств. На каждом $\mathscr{C}^{\vartheta}$ вводится борелевская мера $\lambda^{\vartheta}$, являюшаяся произведением $\vartheta$ экземпляров меры $\boldsymbol{\lambda}$. Борелевские множества этих пространств допускают кодировку, которая может быть определена так. Зафиксируем раз навсегда последовательность $\left\{b_{\vartheta}\right\}_{\vartheta<\omega_{1}^{\mathbf{L}}} \in \mathbf{L}$ биекций $b_{\vartheta}: \mathbb{N} \stackrel{\text { на }}{\longrightarrow} \mathbb{N} \times \xi$. Каждая $b_{\vartheta}$ порождает гомеоморфизм $H_{\vartheta}: 2^{\omega} \stackrel{\text { на }}{\longrightarrow} \mathscr{C}^{\vartheta}$. Если $c \in \mathbf{B C}$, то положим $\boldsymbol{B}_{c}\left[\mathscr{C}^{\vartheta}\right]=H_{\vartheta}$ " $\boldsymbol{B}_{c}$. В смысле этой кодировки, идеал $\mathscr{I}_{\boldsymbol{\lambda}}\left[\mathscr{C}^{\vartheta}\right]$ всех борелевских множеств $X \subseteq \mathscr{C}^{\vartheta}$ таких, что $\boldsymbol{\lambda}^{\vartheta}(X)=0$, является $\mathbf{L}$-абсолютным $\sigma$-УСА-идеалом в борелевской алгебре пространства $\mathscr{C}^{\vartheta}$ при любом $\vartheta<\omega_{1}^{\mathrm{L}} \cdot{ }^{35}$

Положим, для $\vartheta<\omega_{1}^{\mathbf{L}}, \mathbb{P}^{\vartheta}=\left\{p \in \mathbf{B C} \cap \mathbf{L}: \boldsymbol{\lambda}^{\vartheta}\left(\boldsymbol{B}_{p}\left[\mathscr{C}^{\vartheta}\right]\right)>0\right\}$. Каждое $p \in \mathbb{P}^{\vartheta}$ следует "визуализировать" как множество $\boldsymbol{B}_{p}\left[\mathscr{C}^{\vartheta}\right]=H_{\vartheta}$ " $\boldsymbol{B}_{p} \subseteq \mathscr{C}^{\vartheta}$, а не как $\boldsymbol{B}_{p} \subseteq \mathscr{C}$. Соответственно, определим $p \leqslant q$ (т.е. $p$ сильнее), когда $\boldsymbol{B}_{p}\left[\mathscr{C}^{\bar{\vartheta}}\right] \subseteq \boldsymbol{B}_{q}\left[\mathscr{C}^{\vartheta}\right]$.

Наконец, определим $\mathbb{P}=\bigcup_{\vartheta<\omega_{1}^{\mathbf{L}}}\left\{\langle\vartheta, p\rangle: p \in \mathbb{P}^{\vartheta}\right\}$. Порядок на $\mathbb{P}$ вводится так: $\langle\vartheta, p\rangle \leqslant\langle\xi, q\rangle$ (т.е. $\langle\vartheta, p\rangle$ сильнее), когда $\xi \leqslant \vartheta$ и $\boldsymbol{B}_{p}\left[\mathscr{C}^{\vartheta}\right]\left\lceil\xi \subseteq \boldsymbol{B}_{q}\left[\mathscr{C}^{\xi}\right]\right.$, где $\boldsymbol{B}_{p}\left[\mathscr{C}^{\vartheta}\right]\lceil\xi=$ $\left\{\boldsymbol{x}\left\lceil\xi: \boldsymbol{x} \in \boldsymbol{B}_{p}\left[\mathscr{C}^{\vartheta}\right]\right\}\right.$. Опять каждое “условие" $\langle\vartheta, p\rangle \in \mathbb{P}$ "визуализируется" как множество $\boldsymbol{B}_{p}\left[\mathscr{C}^{\vartheta}\right] \subseteq \mathscr{C}^{\vartheta}$.

Фиксируем, для доказательства теоремы 8.1(5), $\mathbb{P}$-генерическое над $\mathbf{L}$ множество $G \subseteq \mathbb{P}$. Положим $G^{\vartheta}=\{p:\langle\vartheta, p\rangle \in G\} ; G^{\vartheta} \subseteq \mathbb{P}^{\vartheta}$. Аналогично лемме 4.11, сушествует единственная “точка" $\boldsymbol{a} \in \mathscr{N}^{\omega_{1}^{\mathbf{L}}}$ (т.е. $\left.\boldsymbol{a}: \omega_{1}^{\mathbf{L}} \rightarrow \mathbb{N}^{\omega}\right)$ такая, что $\boldsymbol{a}\left\lceil\vartheta \in \boldsymbol{B}_{p}\left[\mathscr{C}^{\vartheta}\right]\right.$ всякий раз, когда $\langle\vartheta, p\rangle \in G$. Другими словами, если $\vartheta<\omega_{1}^{\mathbf{L}}$, то $\boldsymbol{a}\lceil\vartheta$ - единственная точка пересечения $\bigcap_{p \in G^{\vartheta}} \boldsymbol{B}_{p}\left[\mathscr{C}^{\vartheta}\right]$.

\footnotetext{
${ }^{34}$ Итерации с конечной поддержкой не годятся для этой цели, поскольку они производят расширения с коэновскими точками $\mathbb{N}^{\omega}$, что ведет к $\mathrm{BP}\left(\boldsymbol{\Delta}_{2}^{1}\right)$ согласно следствию 3.4 .

${ }^{35}$ Мы отвлекаемся от некоторого несоответствия с ситуацией, о которой идет речь в п. 3 Д, надеясь, что читатель сделает, в общем, очевидные поправки.
} 
Мы утверждаем, что в $\mathbf{L}[G]$ истинно $\operatorname{LM}\left(\boldsymbol{\Delta}_{2}^{1}\right)$, но ложны $\operatorname{LM}\left(\boldsymbol{\Sigma}_{2}^{1}\right)$ и $\operatorname{BP}\left(\boldsymbol{\Delta}_{2}^{1}\right)$. Cогласно теореме 3.3 достаточно проверить, что следующие три предложсения истинньг в $\mathbf{L}[G]$ при любом $a \in \mathbf{L}[G] \cap \mathbb{N}^{\omega}$ :

(a) $\operatorname{Rand} \mathrm{L}[a] \neq \varnothing$;

(б) Rand L $[a]$ не является множеством $\lambda$-меры 1 ;

(в) $\operatorname{Coh} \mathrm{L}[a]=\varnothing$.

ЛЕмма 8.12. $\mathbb{P}$ удовлетворяет УСА в $\mathbf{L}$, а потому все кардиналь $\mathbf{L}$ остаются кардиналами в $\mathbf{L}[G]$, в частности, $\omega_{1}^{\mathbf{L}[G]}=\omega_{1}^{\mathbf{L}[\boldsymbol{a}]}=\omega_{1}^{\mathbf{L}}$. Кроме того,

(i) если $x \in \mathbf{L}[G] \cap \mathbb{N}^{\omega}$, то найдется ординал $\xi<\omega_{1}^{\mathbf{L}}$ такой, что $x \in \mathbf{L}[\boldsymbol{a}\lceil\xi]$;

(ii) если $\vartheta<\omega_{1}^{\mathrm{L}}$, то $G^{\vartheta}$ является $\mathbb{P}^{\vartheta}$-генерическим над $\mathbf{L}$;

(iii) если $\vartheta<\omega_{1}^{\mathbf{L}}$, то точка $\boldsymbol{a}\left\lceil\vartheta\right.$ является $\boldsymbol{\lambda}^{\vartheta}$-случайной над $\mathbf{L}$ в том смысле, что $\boldsymbol{a}\left\lceil\vartheta \notin \boldsymbol{B}_{c}\left[\mathscr{C}^{\vartheta}\right]\right.$ всякий раз, когда $c \in \mathbf{B C} \cap \mathbf{L}$ и $\boldsymbol{\lambda}^{\vartheta}\left(\boldsymbol{B}_{c}\left[\mathscr{C}^{\vartheta}\right]\right)=0$;

(iv) если $\vartheta<\omega_{1}^{\mathrm{L}}$, то точка $\boldsymbol{a}(\vartheta)$ является случайной над $\mathbf{L}[\boldsymbol{a}\lceil\vartheta]$, m.е. $\boldsymbol{a}(\vartheta) \notin$ $\boldsymbol{B}_{c}$ всякий раз, когда $c \in \mathbf{B C} \cap \mathbf{L}\left[\boldsymbol{a}\lceil\vartheta]\right.$ u $\boldsymbol{\lambda}\left(\boldsymbol{B}_{c}\right)=0$.

ДокАЗАТЕльство. УСА для $\mathbb{P}$ следует из того, что иначе в $\mathbf{L}$ существовало бы несчетное семейство борелевских подмножеств $\mathscr{C}_{1}^{\omega_{1}^{\mathbf{L}}}$ положительной $\boldsymbol{\lambda}^{\omega_{1}^{\mathbf{L}}}$-меры, имеющих попарные пересечени $\boldsymbol{\lambda}^{\omega_{1}^{\mathbf{L}}}$-меры 0, чего не может быть.

(i) Это следует из счетности антищепей, как обычно.

(ii) Доказательство состоит в проверке того, что если множество $D \subseteq \mathbb{P}^{\vartheta}$ плотно в $\mathbb{P}^{\vartheta}$, то множество $D^{\prime}=\left\{\langle\xi, p\rangle \in \mathbb{P}: \xi \geqslant \vartheta \wedge \exists q \in D\left(\boldsymbol{B}_{p}\left[\mathscr{C}^{\xi}\right]\left\lceil\vartheta=\boldsymbol{B}_{q}\left[\mathscr{C}^{\vartheta}\right]\right)\right\}\right.$ плотно в $\mathbb{P}$ (и если $D \in \mathbf{L}$, то $D^{\prime} \in \mathbf{L}$ ). Отсюда следует (iii) (в сушности, это лемма 4.12 в несколько иной ситуации).

(iv) Можно считать, что на самом деле $\boldsymbol{B}_{c}$ есть $\mathbf{G}_{\delta}$-множество, т.е. $\boldsymbol{B}_{c}=\mathbf{U}_{z}=$ $\bigcap_{n} \bigcup_{z\left(2^{n} \cdot 3^{m}\right)=0} \mathscr{N}_{s_{m}}$ для подходяшего $z \in \mathbf{L}\left[\boldsymbol{a}\lceil\vartheta] \cap \mathbb{N}^{\omega}\right.$. Мы имеем по теореме 2.6(i) $z=\boldsymbol{f}_{\xi}\left[H_{\vartheta}{ }^{-1}(\boldsymbol{a}\lceil\vartheta)]\right.$ для какого-то $\vartheta<\omega_{1}^{\mathbf{L}}$. Пусть напротив, $\boldsymbol{a}(\vartheta) \in \mathbf{U}_{z}$. Тогда

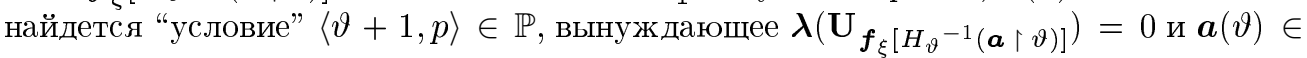
$\mathbf{U}_{\boldsymbol{f}_{\xi}\left[H_{\vartheta}{ }^{-1}(\boldsymbol{a}\lceil\vartheta)]\right.}$. (Мы, как это часто делается, отождествляем множества вроде $\boldsymbol{a}(\vartheta)$ и $\boldsymbol{a}\left\lceil\vartheta\right.$ в генерических расширениях с их именами.) Положим $P=\boldsymbol{B}_{p}\left[\mathscr{C}^{\vartheta+1}\right]$; тогда, очевидно, $\boldsymbol{\lambda}^{\vartheta+1}(P)>0$. Кроме того, по выбору $p$, множество $X=\{\boldsymbol{x} \in P\lceil\vartheta$ : $\left.\boldsymbol{\lambda}\left(\mathbf{U}_{\boldsymbol{f}_{\xi}\left[H_{\vartheta}-1(\boldsymbol{x})\right]}\right)>0\right\}$ имеет $\boldsymbol{\lambda}^{\vartheta}$-меру 0 (аналог леммы 4.13).

Следовательно, по соображениям, связанным с теоремой Фубини, найдется более сильное "условие" $\langle\vartheta+1, q\rangle \in \mathbb{P}$ (т.е. множество $Q=\boldsymbol{B}_{q}\left[\mathscr{C}^{\vartheta+1}\right]$ удовлетворяет $Q \subseteq P$ ) такое, что сечение $Q_{\boldsymbol{x}}=\left\{y \in \mathbb{N}^{\omega}:\langle\boldsymbol{x}, y\rangle \in Q\right\}$ не пересекается с $\mathbf{U}_{\boldsymbol{f}_{\xi}\left[H_{\vartheta}{ }^{-1}(\boldsymbol{x})\right]}$, каково бы ни было $\boldsymbol{x} \in Y=Q\left\lceil\vartheta\right.$. Но такое $Q$ вынуждает $a_{\vartheta} \notin \mathbf{U}_{\boldsymbol{f}_{\xi}\left[H_{\vartheta}{ }^{-1}(\boldsymbol{a}\lceil\vartheta)]\right.}$ (например, по лемме 4.13 , точнее, ее аналогу для данной ситуации), что противоречит выбору "условия" $\langle\vartheta+1, p\rangle$.

Теперь следуют доказательства утверждений (а), (б), (в).

(a) Пусть $a \in \mathbf{L}[G] \cap \mathbb{N}^{\omega}$. Имеем $a \in \mathbf{L}\left[\boldsymbol{a}\lceil\vartheta]\right.$ для некоторого $\vartheta<\omega_{1}^{\mathbf{L}}$ по лемме 8.12(i). Но тогда $a_{\vartheta} \in \mathbf{R}$ and $\mathbf{L}[a]$ по лемме 8.12(iv).

(б) Достаточно убедиться, что $\mathbf{L} \cap \mathbb{N}^{\omega}$ не является множеством $\boldsymbol{\lambda}$-меры 0 в $\mathbf{L}[G]$. Для этого нужно показать, что $\mathbf{L} \cap \mathbb{N}^{\omega}-$ не множество меры 0 в $\mathbf{L}\left[\boldsymbol{a}\lceil\vartheta]\right.$ для любого $\vartheta<\omega_{1}^{\mathbf{L}}$. Предположим противное. Тогда найдется $z \in \mathbf{L}\left[\boldsymbol{a}\lceil\vartheta] \cap \mathbb{N}^{\omega}\right.$ такое, что $\mathbf{L} \cap \mathbb{N}^{\omega} \subseteq \mathbf{U}_{z}$ и $\boldsymbol{\lambda}\left(\mathbf{U}_{z}\right)=0$. Имеем $z=\boldsymbol{f}_{\xi}\left[H_{\vartheta}{ }^{-1}(\boldsymbol{a}\lceil\vartheta)]\right.$ для какого-то $\xi<\omega_{1}^{\mathbf{L}}$. Противоречие 
будет получено из рассмотрения сечений $P_{\boldsymbol{x}}=\{y: P(\boldsymbol{x}, y)\}$ и $P^{y}=\{\boldsymbol{x}: P(\boldsymbol{x}, y)\}$ следующего $\Sigma_{1}^{1}(\mathbf{L})$-множества:

$$
P=\left\{\langle\boldsymbol{x}, y\rangle \in \mathscr{N}^{\vartheta} \times \mathbb{N}^{\omega}: y \in \mathbf{U}_{\boldsymbol{f}_{\xi}\left[H_{\vartheta}{ }^{-1}(\boldsymbol{x})\right]}\right\} .
$$

По определению, $P_{\boldsymbol{a}\lceil\vartheta}=\mathbf{U}_{z}$ является множеством $\boldsymbol{\lambda}$-меры 0 и $\mathbf{L} \cap \mathbb{N} \omega \subseteq P_{\boldsymbol{a}} \mid \vartheta$. Но это предложение - абсолютная формула по теореме 2.8 ( “ $\boldsymbol{\lambda}\left(\mathbf{U}_{z}\right)=0$ " - арифметическая формула), поэтому оно релятивизуемо к $\mathbf{L}[\boldsymbol{a}\lceil\vartheta]$, тем самьм, оно вьнуждается в $\mathbf{L}$ некоторым условием $\langle\vartheta, p\rangle \in \mathbb{P}^{\vartheta}$ таким, что $\boldsymbol{a}\left\lceil\vartheta \in \boldsymbol{B}_{p}\left[\mathscr{C}^{\vartheta}\right]\right.$ (лемма 4.12 для идеала множеств $\boldsymbol{\lambda}^{\vartheta}$-меры 0). Тогда $p \in \mathbf{B C} \cap \mathbf{L}$ и $X=\boldsymbol{B}_{p}\left[\mathscr{C}^{\vartheta}\right]-$ множество положительной $\boldsymbol{\lambda}^{\vartheta}$-меры. Лемма 4.13 , по выбору $p$, дает следующее в $\left.\mathbf{L}: 1\right)$ для любого $y \in \mathbb{N}^{\omega}$ множество $\boldsymbol{B}_{p}\left[\mathscr{C}^{\vartheta}\right] \backslash P^{y}$ имеет положительную $\boldsymbol{\lambda}$-меру, но 2) $\boldsymbol{\lambda}\left(P_{\boldsymbol{x}}\right)=0$ для почти всех (с точностью до $\boldsymbol{\lambda}^{\vartheta}$-меры 0) точек $\boldsymbol{x} \in \boldsymbol{B}_{p}\left[\mathscr{C}^{\vartheta}\right]$. Имеем противоречие с теоремой Фубини.

(в) Рассуждения, подобные изложенным вьше, приводят проблему к виду: 1) если $\vartheta<\omega_{1}^{\mathbf{L}}$ и $x \in \mathbf{L}\left[\boldsymbol{a}\lceil\vartheta]\right.$, то найдется код $c \in \mathbf{B C} \cap \mathbf{L}[a]$ такой, что $\boldsymbol{B}_{c}$ - тошее множество и $x \in \boldsymbol{B}_{c}$, а затем к виду: 2) если $X \subseteq \mathbb{N}^{\omega}$ - борелевское множество с $\boldsymbol{\lambda}(X)>0$, а $F: X \rightarrow \mathbb{N}^{\omega}$ - борелевская функция, то найдется борелевское $X^{\prime} \subseteq X$, снова с $\boldsymbol{\lambda}\left(X^{\prime}\right)>$ 0 , такое, что $F^{\prime \prime} X^{\prime}$ - тошее множество. Понятно, что для всякого $\varepsilon>0$ и $s \in \mathbb{N}<\omega$ найдется $s^{\prime} \in \mathbb{N}^{<\omega}$ такое, что $s \subseteq s^{\prime}, \lambda\left(F^{-1}\left(\mathscr{N}_{s^{\prime}}\right)\right)<\varepsilon$. Следовательно, найдется плотное открытое множество $Y \subseteq \mathbb{N}^{\omega}$ с $\boldsymbol{\lambda}\left(F^{-1}(Y)\right)<\varepsilon$. Остается определить $X^{\prime}=$ $X \backslash\left(F^{-1}(Y)\right)$.

Исторические и библиографические замечания. История результатов, изложенных в этом параграфе, растягивается с конца 60-х годов до середины 90-х годов прошлого века и, в общем, связана с развитием методов форсинга и итерированного форсинга. Исходными ссылками по итерированному форсингу являются [12], [90], см. также книги вроде [10], [29], [30], [44]. На русском языке подходяших источников нет, кроме $[11 ;$ гл. 4], где рассмотрены итерации длины 2 , а также итерации произвольной длины, но в том частном случае, когда целью является непротиворечивость аксиомы Мартина.

Утверждение $\operatorname{LM}\left(\boldsymbol{\Sigma}_{2}^{1}\right) \not \operatorname{PK}\left(\boldsymbol{\Pi}_{1}^{1}\right)$, как мы упоминали, следует из ранних работ по аксиоме Мартина. Результат независимо получен Любецким (см. [65], [66], [68]; доказательство через итерацию форсинга $\mathbf{A}_{\varepsilon}$ дано в [69]).

Утверждение $\operatorname{BP}\left(\boldsymbol{\Sigma}_{2}^{1}\right) \wedge \operatorname{LM}\left(\boldsymbol{\Delta}_{2}^{1}\right) \not \operatorname{LM}\left(\boldsymbol{\Sigma}_{2}^{1}\right)$ представляет собой теорему 9.3.6 в [10], данную там со ссылкой на статью [26], где такого результата, однако, нет.

Что касается $\mathrm{BP}\left(\boldsymbol{\Sigma}_{2}^{1}\right) \not \operatorname{LM}\left(\boldsymbol{\Delta}_{2}^{1}\right)$, метод итерации доминирующего форсинга, как в п. 8В, взят нами из [10; 9.3.5] (где опять приведена ложная ссылка на [26]). Однако еще раньше Стерн [92] показал даже $\operatorname{BP}\left(\boldsymbol{\Sigma}_{\infty}^{1}\right) \not \operatorname{LM}\left(\boldsymbol{\Delta}_{2}^{1}\right)$ (но при помоши более сложной модели, чем рассмотренная в п. $8 \mathrm{~B})$.

Мы не смогли найти результат $\operatorname{BP}\left(\boldsymbol{\Delta}_{2}^{1}\right) \wedge \mathrm{LM}\left(\boldsymbol{\Delta}_{2}^{1}\right) \not \Longrightarrow \mathrm{BP}\left(\boldsymbol{\Sigma}_{2}^{1}\right)$ сфорормлированным где-либо в явном виде, однако представленное нами доказательство представляет собой определенную комбинацию методов, известных, например, из [10].

Утверждение $\operatorname{LM}\left(\boldsymbol{\Delta}_{2}^{1}\right) \neq \mathrm{BP}\left(\boldsymbol{\Delta}_{2}^{1}\right)$ доказано в [69]. Отметим, что симметричное утверждение $\operatorname{BP}\left(\boldsymbol{\Delta}_{2}^{1}\right) \not \operatorname{LM}\left(\boldsymbol{\Delta}_{2}^{1}\right)$ (более слабое, конечно, чем доказанное вьше в этом параграфе $\left.\operatorname{BP}\left(\boldsymbol{\Sigma}_{2}^{1}\right) \not \operatorname{LM}\left(\boldsymbol{\Delta}_{2}^{1}\right)\right)$ было, вероятно, известно к 1968 г., поскольку оно легко выводится из теоремы [74; Т 3323] (данной там со ссылкой на Кюнена и Соловея) о том, что присоединение коэновских точек в любом числе к конструктивному универсуму $\mathbf{L}$ дает модель, в которой есть неизмеримое $\boldsymbol{\Delta}_{2}^{1}$-множество. 


\section{§. Дальнейшие результаты}

В этом заключительном параграфе содержится обзор результатов о свойствах регулярности проективных множеств, касающихся таких вопросов, как роль аксиомы существования недостижимого кардинала, свойства регулярности множеств начиная с третьего проективного уровня и некоторые менее традиционные свойства регулярности. Размер статьи заставляет нас ограничиться здесь лишь обзором главных результатов без доказательств, но со ссылками на оригинальные работы.

9А. Нужен ли недостижимый кардинал? Мы видели, что аксиома существования (строго) недостижимого кардинала используется в доказательстве непротиворечивости предположения о том, что все проективные множества имеют каждое из трех свойств регулярности. Эта аксиома - довольно сильное утвержение, заведомо недоказуемое в ZFC (из него даже следует непротиворечивость ZFC), а потому возникает естественньй вопрос: насколько эта гипотеза действительно необходима для достижения указанного результата.

Наиболее простой ответ получается для свойства совершенного ядра. Действительно, если каждое несчетное даже $\boldsymbol{\Pi}_{1}^{1}$-множество содержит совершенное подмножество, то, по теореме $3.3, \mathbf{L}[a] \cap \mathbb{N}^{\omega}$ счетно, т.е. $\omega_{1}^{\mathbf{L}[a]}<\omega_{1}$ для каждого $a \in \mathbb{N}^{\omega}$. Отсюда легко следует, что $\aleph_{1}$ - недостижимьй кардинал в любом универсуме вида $\mathbf{L}[a], a \in \mathbb{N}^{\omega}$, в частности, в $\mathbf{L}$, или, другими словами, если утверждение $\forall a \in \mathbb{N}^{\omega}$ $\left(\omega_{1}^{\mathbf{L}[a]}<\omega_{1}\right)$ не противоречит $\mathbf{Z F C}$, то сушествование строго недостижимого кардинала также непротиворечиво (отмечено Любецким в [17]). Таким образом, в отношении доказательства непротиворечивости свойства совершенного ядра даже для $\boldsymbol{\Pi}_{1}^{1}$-множеств использование недостижимых кардиналов совершенно необходимо.

Вопрос о роли недостижимого кардинала для проблем LM и ВР оказался значительно более сложным. Ответ различается для меры и категории:

Теорема 9.1 (Шелах [84], Рэзонье [79]). Из непротиворечивости ZFC следует непротиворечивость $\mathbf{Z F C}+\operatorname{BP}\left(\boldsymbol{\Sigma}_{\infty}^{1}\right)$, и даже $\mathbf{Z F C}+\mathrm{BP}(\mathbf{R O D})$.

$B$ то жее время, любое из двух предположений $\operatorname{LM}\left(\Sigma_{3}^{1}\right), \operatorname{LM}\left(\Sigma_{2}^{1}\right) \wedge \operatorname{BP}\left(\Sigma_{3}^{1}\right)$ влечет, что $\aleph_{1}$ - недостижимый кардинал в $\mathbf{L}$ и в $\mathbf{L}[a]$ для любого $а \in \mathbb{N} \omega, u$ тем самым влечет $\mathrm{PK}\left(\boldsymbol{\Pi}_{1}^{1}\right)$ по теореме $3.3(\mathrm{i})$.

Таким образом, аксиома недостижимого кардинала необходима для положительного решения проблемы измеримости для проективных (даже $\boldsymbol{\Sigma}_{3^{-}}^{1}$ ) множеств, но без нее можно обойтись для положительного решения проблемы свойства Бэра. Впрочем, как показал Стерн [94], непротиворечивость LM(OD) (для ординально определимых множеств!) не требует недостижимого кардинала.

Аксиома Мартина МА (см. [11; гл. 6]) сушественно усиливает некоторые формы гипотез LM и ВР. Например, в предположении $\mathbf{M} \mathbf{A}_{\aleph_{1}}$, недостижимость $\aleph_{1}$ в $\mathbf{L}[a]$, $a \in \mathbb{N}^{\omega}$, следует уже из $\operatorname{LM}\left(\boldsymbol{\Delta}_{3}^{1}\right)$, а также из $\mathrm{BP}\left(\boldsymbol{\Delta}_{3}^{1}\right)$, см. [33]. (Без $\mathbf{M} \mathbf{A}_{\aleph_{1}}$ это не имеет места согласно теоремам 9.1 и 9.2.) Однако на самом деле непротиворечивость теории $\mathbf{Z F C}+\mathbf{M} \mathbf{A}_{\aleph_{1}}+\operatorname{LM}\left(\boldsymbol{\Delta}_{3}^{1}\right)$ (или, вместо последнего слагаемого, $\left.\mathrm{BP}\left(\boldsymbol{\Delta}_{3}^{1}\right)\right)$ влечет непротиворечивость существования даже такого большого кардинала, как суперкомпактньй $[21],[27]$.

9Б. Проблемы третьего и четвертого проективных уровней. Взаимоотношения между гипотезами $\operatorname{LM}\left(\boldsymbol{\Sigma}_{2}^{1}\right), \operatorname{BP}\left(\boldsymbol{\Sigma}_{2}^{1}\right), \operatorname{LM}\left(\boldsymbol{\Delta}_{2}^{1}\right), \operatorname{BP}\left(\boldsymbol{\Delta}_{2}^{1}\right)$ в ZFC исчерпываюшим образом решаются следствием 3.5 и теоремой 5.4 (в части доказуемых связей 
между ними) и теоремой 8.1 (в части недоказуемых связей). Для третьего проективного уровня такой полной картины нет. Тем не менее, удалось доказать следующее.

Tеорема 9.2. (1) (Шелах [84]) Из непротиворечивости ZFC следует непротиворечивость $\mathbf{Z F C}+\mathrm{BP}\left(\boldsymbol{\Sigma}_{3}^{1}\right)$.

(2) (Джюдах [32]) $\operatorname{LM}\left(\boldsymbol{\Delta}_{3}^{1}\right) \neq \mathrm{BP}\left(\boldsymbol{\Delta}_{3}^{1}\right)$.

(3) (Джюдах [31], [32]) $\mathrm{BP}\left(\Delta_{3}^{1}\right) \not \operatorname{LM}\left(\Delta_{3}^{1}\right)$, и даже $\mathrm{BP}\left(\Delta_{3}^{1}\right) \not \operatorname{LM}\left(\Delta_{2}^{1}\right)$.

В этой теореме $A \not B$ означает, что импликаци $A \Longrightarrow B$ недоказуема в $\mathbf{Z F C}$ (в предположении, что эта теория непротиворечива).

Комментарии к теореме.

(1) Наиболее простая модель для $\operatorname{BP}\left(\Sigma_{3}^{1}\right)$ описана в [10; с. 470]: это итерированное расширение класса $\mathbf{L}$ c конечной базой длины $\omega_{1}^{\mathbf{L}}$, в котором на предельных шагах применяется $\varepsilon$-случайный форсинг, а на непредельных шагах, по очереди, случайный и коэновский форсинги. Другая модель для $\mathrm{BP}\left(\boldsymbol{\Sigma}_{3}^{1}\right)$ представлена в [27]; в ней, кроме того, аксиома Мартина $\mathbf{M} \mathbf{A}_{\aleph_{1}}$ верна для достаточно широкого класса ЧУ множеств.

(2) Эта часть теоремы характеризуется в [34], [6] как совместньй результат Багария и Джюдаха. Модель, описанная в [10; теорема 9.4.19], выглядит так: мы начинаем с модели для $\mathbf{M A}+\left(\omega_{1}=\omega_{1}^{\mathbf{L}}\right)$, а затем используем форсинг, описанный в п. $8 \mathrm{E}$ (присоединение $\aleph_{1}$ случайных точек). Более простая модель, использующая, однако, измеримый кардинал, указана в [33].

(3) В сушности, может быть применена модель из п. 8В (итерация доминирующего форсинга), поскольку, как показано в [10; теорема 9.4.7], в этой модели истинно не только $\mathrm{BP}\left(\boldsymbol{\Sigma}_{2}^{1}\right)$, но и $\mathrm{BP}\left(\boldsymbol{\Delta}_{3}^{1}\right)$.

Для четвертого проективного уровня первый нетривиальный результат о свойствах регулярности был получен Джюдахом и Спинасом [34]: $\operatorname{LM}\left(\boldsymbol{\Delta}_{4}^{1}\right) \nRightarrow \operatorname{BP}\left(\boldsymbol{\Delta}_{4}^{1}\right)$. (Доказательство использует аксиому о недостижимом кардинале, чего в этом случае избежать нельзя, поскольку уже $\operatorname{LM}\left(\boldsymbol{\Sigma}_{3}^{1}\right)$ влечет существование недостижимого кардинала по теореме 9.1.) Невыводимость обратной импликации здесь очевидна: согласно теореме 9.1 , вьполняется даже $\mathrm{BP}\left(\Delta_{\infty}^{1}\right) \not \mathrm{LM}\left(\Sigma_{3}^{1}\right)$. О дальнейших исследованиях в этом направлении см. [6].

Для пятого и более высоких уровней никаких заслуживаюших внимания результатов не появилось.

9В. Некоторые новые $\sigma$-УСА-идеалы и другие свойства регулярности. Помимо меры и категории польских топологий, еще одним регулярным методом получения $\sigma$-УСА-идеалов яВляются не-польские УСА-топологии. Для поддержки единства терминологии, назовем $\sigma$-УСА-топологией такую топологию, которая удовлетворяет второй аксиоме счетности (т.е. нет несчетных семейств попарно непересекаюшихся непустых открытых множеств) и является бэровской (т.е. непустые открытые множества не могут быть тощими). Сюда относится, например, польская топология $\mathbb{N}^{\omega}$ или любого другого польского пространства, в частности, обычная топология вещественной прямой.

Богатьм источником $\sigma$-УСА-топологий являются УСА-форсинги. Рассмотрим, к примеру, доминируюший форсинг $\mathbf{D}$. Каждому “условию" $p=\langle s, f\rangle \in \mathbf{D}$ сопоставляется множество

$$
U_{p}=U_{f}^{s}=\left\{x \in \mathbb{N}^{\omega}: s \subset x \wedge \forall n(x(n) \geqslant f(n))\right\} .
$$


Семейство всех множеств вида $U_{p}, p \in \mathbf{D}$, замкнуто относительно конечных объединений, вследствие чего его можно взять за базу топологии на $\mathbb{N} \omega$, которую мы обозначим через $\mathscr{T}_{\mathbf{D}}$. Вторая аксиома счетности для $\mathscr{T}_{\mathbf{D}}$ следует из УCA для $\mathbf{D}$, а бэровость из того, что пересечение убывающей системы множеств $U_{f_{0}}^{s_{0}} \supseteq U_{f_{1}}^{s_{1}} \supseteq \cdots \supseteq U_{f_{n}}^{s_{n}} \supseteq \ldots$ такой, что $\operatorname{lh} s_{n} \rightarrow \infty$, содержит точку $x=\bigcup_{n} s_{n}$. Таким образом, идеал $\mathscr{J} \mathbf{D}$ всех $\mathscr{T}_{\mathbf{D}}$-тощих борелевских множеств $X \subseteq \mathbb{N}^{\omega}$ является $\sigma$-УСА-идеалом.

ЛЕмма 9.3. $\operatorname{cod} \mathscr{J}_{\mathbf{D}}=\left\{c \in \mathbf{B C}: \boldsymbol{B}_{c} \in \mathscr{J}_{\mathbf{D}}\right\}$ есть $\Sigma_{2}^{1}$-множество.

ДокАЗАТЕльство. Рассматривая двойные последовательности (“матрицы”) вида $\left\{\left\langle s_{k n}, f_{k n}\right\rangle\right\}_{k, n \in \mathbb{N}}$ как точки соответствуюшего пространства, мы имеем

$$
\begin{aligned}
\boldsymbol{B}_{c} \in \mathscr{J}_{\mathbf{D}} \Longleftrightarrow & \exists\left\{\left\langle s_{k n}, f_{k n}\right\rangle\right\}\left(\forall k, n\left(\left\langle s_{k n}, f_{k n}\right\rangle \in \mathbf{D}\right) \wedge \forall k\left(\boldsymbol{B}_{c} \cap \bigcup_{n} U_{f_{k n}}^{s_{k n}}=\varnothing\right)\right. \\
& \left.\forall k \forall\langle s, f\rangle\left(\langle s, f\rangle \in \mathbf{D} \Longrightarrow \exists n\left(U_{f_{k n}}^{s_{k n}} \cap U_{f}^{s} \neq \varnothing\right)\right)\right) .
\end{aligned}
$$

Здесь формулы $\left\langle s_{k n}, f_{k n}\right\rangle \in \mathbf{D}$ и $U_{f_{k n}}^{s_{k n}} \cap U_{f}^{s} \neq \varnothing$ являются арифметическими, а формула $\boldsymbol{B}_{c} \cap \bigcup_{n} U_{f_{k n}}^{s_{k n}}=\varnothing$ легко приводится к $\Pi_{1}^{1}$-виду при помоши формулы $\sigma$ предложения $1.11(\mathrm{iv})$, так что результат становится $\Sigma_{2}^{1}$, что и требовалось.

Таким образом, $\mathscr{J}_{\mathbf{D}}$ является $\mathbf{L}$-абсолютным $\sigma$-УСА-идеалом. Это означает, что к $\mathscr{J} \mathbf{D}$ применима теорема 3.9. Отсюда следует:

$$
\begin{aligned}
& \mathrm{M}_{\mathscr{J}_{\mathbf{D}}}\left(\boldsymbol{\Sigma}_{2}^{1}\right) \Longleftrightarrow \forall a \in \mathbb{N}^{\omega}\left(\mathbf{R a n d}_{\mathscr{J}_{\mathbf{D}}} \mathbf{L}[a]-\text { не } \mathscr{I}_{\text {-полное множество }) ;}\right. \\
& \mathrm{M}_{\mathscr{J}_{\mathbf{D}}}\left(\boldsymbol{\Delta}_{2}^{1}\right) \Longleftrightarrow \forall a \in \mathbb{N}^{\omega} \forall c \in \mathbf{L}[a] \cap \mathbf{B C}\left(\boldsymbol{B}_{c} \notin \mathscr{J}_{\mathbf{D}} \Longrightarrow \mathbf{R a n d}_{\mathscr{J}_{\mathbf{D}}} \mathbf{L}[a] \cap \boldsymbol{B}_{c} \neq \varnothing\right) .
\end{aligned}
$$

Нетрудно проверить, что к идеалу $\mathscr{J} \mathbf{D}$ применимо доказательство леммы $3.2(1)$, что позволяет переписать вторую эквивалентность так:

$$
\mathrm{M}_{\mathscr{J}_{\mathbf{D}}}\left(\Delta_{2}^{1}\right) \Longleftrightarrow \forall a \in \mathbb{N}^{\omega}\left(\text { Rand }_{\mathscr{J}_{\mathbf{D}}} \mathbf{L}[a] \neq \varnothing\right)
$$

Значение и смысл свойства $\mathscr{J} \mathbf{D}^{-и з м е р и м о с т и ~ и ~ г и п о т е з ~} \mathrm{M}_{\mathscr{J}_{\mathbf{D}}}\left(\boldsymbol{\Sigma}_{2}^{1}\right)$ и $\mathrm{M}_{\mathscr{J}_{\mathbf{D}}}\left(\boldsymbol{\Delta}_{2}^{1}\right)$ далеко не столь ясны, как для идеалов $\mathscr{I}_{\text {cat }}$ и $\mathscr{I}_{\boldsymbol{\lambda}}$. Тем более интересными представляются следуюшие результаты [14]:

(i) $\mathrm{M}_{\mathscr{J}_{\mathbf{D}}}\left(\boldsymbol{\Delta}_{2}^{1}\right)$ эквивалентно $\mathrm{BP}\left(\boldsymbol{\Sigma}_{2}^{1}\right)$;

(ii) $\mathrm{M}_{\mathscr{J}_{\mathbf{D}}}\left(\boldsymbol{\Sigma}_{2}^{1}\right)$ эквивалентно $\forall a \in \mathbb{N}^{\omega}\left(\omega_{1}^{\mathbf{L}[a]}<\omega_{1}^{\mathbf{L}}\right)$, т.е. эквивалентно $\operatorname{PK}\left(\boldsymbol{\Pi}_{1}^{1}\right)$ по теореме $3.3(\mathrm{i})$.

Итак, по заданному форсингу $\mathbf{D}$ мы построили $\mathbf{L}$-абсолютньй $\sigma$-УCA-идеал $\mathscr{J} \mathbf{D}$. Определение 4.8 извлекает из последнего форсинг $\mathbb{P}_{\mathscr{J}}$, которьй дает те же генерические расширения, что и исходньй форсинг $\mathbf{D},-$ и по тем же причинам, что и для форсинга Коэна в п. 4 Г.

Как показано в $[10 ; 3.4 . В$ и 3.7], эту конструкцию $\sigma$-УСА-идеала можно модифицировать так, что она становится применимой к ряду других УСА-форсингов $\mathbb{P}$, не обязательно связанных с какой-либо топологией. Ключевая идея может быть объяснена так. Предположим, что $t$ есть $\mathbb{P}$-имя такое, что $\Vdash_{\mathbb{P}}^{\mathbf{V}}(t \in \mathbb{N} \omega)$. Определим $\mathscr{I}_{\mathbb{P} t}$ как множество всех борелевских множеств вида $\boldsymbol{B}_{p}$ таких, что $\Vdash_{\mathbb{P}}^{\mathbf{V}}\left(t \notin \boldsymbol{B}_{p}\right)$. Если $\mathbb{P}$ есть 
УСА-форсинг, то $\mathscr{I}_{\mathbb{P} t}$ является $\sigma$-УСА-идеалом. Другая модификация этой конструкции применима и к некоторым не-УСА-форсингам, вроде форсинга Сакса. Соответствующие свойства регулярности, уже не обязательно напрямую связанные с тем или иньм идеалом, рассмотрены в [14].

Еще один подход к построению $\sigma$-УСА-идеалов представлен в [81].

В заключение скажем несколько слов еше о двух свойствах регулярности, возникающих из топологических соображений. Множество $X \subseteq \mathbb{N}^{\omega}$ назьвается $\mathbf{K}_{\sigma}$-регулярнblм, если оно либо $\sigma$-компактно, либо содержит суперсовершенное ${ }^{36}$ подмножество. (Ср. со свойством совершенного ядра.) Множество $X \subseteq[\mathbb{N}]^{\omega}=\left\{x \subseteq \mathbb{N}: \operatorname{card} x=\aleph_{0}\right\}$ называется рамсеевским, если найдется такое бесконечное множество $z \subseteq \mathbb{N}$, что $[z]^{\omega} \subseteq X$ или $[z]^{\omega} \cap X=\varnothing$. (Это свойство возникает при рассмотрении некоторых вопросов инфинитарной комбинаторики и в теории моделей.)

Подобно свойствам PK, LM, BP, каждое $\boldsymbol{\Sigma}_{1}^{1}$-множество $\mathbf{K}_{\sigma}$-регулярно и является рамсеевским (см., например, [30], [42]), в то время как на втором проективном уровне возникают неразрешимые проблемы. См. статьи [26], [28] об исследованиях в этом направлении. Для примера, приведем один из полученных результатов: $\mathrm{BP}\left(\boldsymbol{\Sigma}_{2}^{1}\right)$ влечет $\mathbf{K}_{\sigma}$-регулярность всех $\boldsymbol{\Sigma}_{2}^{1}$-множеств, но не наоборот.

\section{СПИСОК ЛИТЕРАТУРЫ}

[1] J. Addison. Separation principles in the hierarchies of classical and effective descriptive set theory // Fund. Math. 1959. V. 46. P. 123-135.

[2] J. Addison. Some consequences of the axiom of constructibility // Fund. Math. 1959. V. 46. P. 337-357.

[3] P. Alexandroff. Sur la puissance des ensembles mesurables B // C. R. Acad. Sci. Paris. 1916. V. 162. Р. 323-325. [Русский перевод: О мощности множеств, измеримых по Борелю // П. С. Александров. Теория функций действительного переменного и теория топологических пространств. М.: Наука, 1978. С. 35-39.]

[4] П. С. Александров. Введение в теорию множеств и общую топологию. М.: Наука, 1977.

[5] В.Я. Арсенин, А.А. Ляпунов. Теория А-множеств // УМН. 1950. Т. 5. №5. C. $45-108$.

[6] J. Bagaria, H. Woodin. $\Delta_{n}^{1}$ sets of reals // J. Symbolic Logic. 1997. V. 62. № 4 . P. 1379-1428.

[7] R. Baire. Sur les fonctions de variables reelles // Ann. Mat. Pura Appl. Ser. IIIa. 1899. V. 3. P. 1-122.

[8] R. Baire, É. Borel, J. Hadamard, H. Lebesgue. Cinq lettres sur la théorie des ensembles // Bull. Soc. Math. France. 1905. V. 33. P. 261-273.

[9] T. Bartoszyński. Additivity of measure implies additivity of category // Trans. Amer. Math. Soc. 1984. V. 281. № 1. P. 209-213.

[10] T. Bartoszyński, H. Judah. Set Theory. On the Structure of the Real Line. Wellesley: A K Peters, 1995.

[11] Дж. Барвайс (ред.). Справочная книга по математической логике. Ч. II: Теория множеств. М.: Наука, 1982.

[12] J. Baumgartner. Iterated forcing // Surveys in Set Theory. Cambridge: Cambridge Univ. Press, 1983. P. 1-59. (London Math. Soc. Lecture Note Ser. V. 87.)

[13] É. Borel. Leçons sur la théorie des fonctions. Paris, 1898.

[14] J. Brendle, B. Löwe. Solovay-type characterizations for forcing-algebras // J. Symbolic Logic. 1999. V. 64. № 3. P. 1307-1323.

[15] П. Дж. Коэн. Теория множеств и континуум-гипотеза. М.: Мир, 1969.

\footnotetext{
${ }^{36}$ Множество назьвается суперсов ериенныц, если оно не замкнуто, не имеет изолированных точек и не является $\sigma$-компактным ни на одном из своих открытых, в топологии, унаследованной из $\mathbb{N}^{\omega}$, подмножеств.
} 
[16] E. van Douwen. The integers and topology // Handbook of Set-Theoretic Topology / ed. K. Kunen, J. Vaughan. Amsterdam: North-Holland, 1984. P. 111-167.

[17] А. Г. Драгалин, В.А.Любецкий. Независимость некоторых проблем аксиоматической теории множеств // Тезисы докладов на Всесоюзном симпозиуме по математической логике. Алма-Ата, 1969. С. 12-17.

[18] P. Du Bois Reymond. Eine neue Theorie der Convergenz und Divergenz von Reihen mit positive Gliedern // J. Reine Angew. Math. 1873. V. 76. P. 61-91.

[19] А. Френкель, И. Бар-Хиллел. Основания теории множеств. М.: Мир, 1966.

[20] К. Гёдель. Совместимость аксиомы выбора и обобщенной континуум-гипотезы с аксиомами теории множеств // УМН. 1948. Т. 3. №1. С. 96-149.

[21] L. Harrington, S. Shelah. Some exact equiconsistency results in set theory // Notre Dame J. Formal Logic. 1985. V. 26. № 2. P. 178-188.

[22] F. Hausdorff. Die Graduirung nach dem Endverlauf // Abh. Königlich Sächs. Ges. Wiss. Leipzig Math.-Phys. 1909. V. 31. P. 295-334.

[23] F. Hausdorff. Die Machtigkeit der Borelschen Mengen // Math. Ann. 1916. V. 77. P. $430-437$.

[24] F. Hausdorff. Summen von $\aleph_{1}$ Mengen // Fund. Math. 1936. V. 26. P. 241-255.

[25] F. Hausdorff. Mengenlehre. Berlin: de Gruyter, 1927. [Русский перевод: Ф. Хаусдорф. Теория множеств / Под ред. и с доп. П. С. Александрова и А.Н. Колмогорова. М.-Л.: ОНТИ, 1937.]

[26] J. Ihoda (H. Judah). $\Sigma_{2}^{1}$-sets of reals // J. Symbolic Logic. 1988. V. 53. № 2. P. 636-642.

[27] J. Ihoda, S. Shelah. Martin's axioms, measurability and equiconsistency results // J. Symbolic Logic. 1989. V. 54. № 1. P. 78-94.

[28] J. Ihoda, S. Shelah. $\Delta_{2}^{1}$-sets of reals // Ann. Pure Appl. Logic. 1989. V. 42. № 3. P. 207-223.

[29] Т. Йех. Теория множеств и метод форсинга. М.: Мир, 1973.

[30] T. Jech. Set Theory. New York: Academic Press, 1978.

[31] H. Judah. Exact equiconsistency results for $\Delta_{3}^{1}$-sets of reals // Arch. Math. Logic. 1992. V. 32. № 2. P. 101-112.

[32] H. Judah. $\Delta_{3}^{1}$-sets of reals // Israel Math. Conf. Proc. 1993. V. 6. P. 361-384.

[33] H. Judah, S. Shelah. $\Delta_{3}^{1}$-sets of reals // J. Symbolic Logic. 1993. V. 58. № 1. P. 72-80.

[34] H. Judah, O. Spinas. On the structure of $\Delta_{4}^{1}$-sets of reals // Arch. Math. Logic. 1995. V. 34. № 5. P. 301-312.

[35] В. Г. Кановей. Проективная иерархия Н. Н. Лузина: современное состояние теории // Справочная книга по математической логике. Ч. II: Теория множеств / ред. Дж. Барвайс. M.: Наука, 1982. С. 273-364.

[36] В. Г. Кановей. Неразрешимые и разрешимые свойства конституант // Матем. сб. 1984. T. 124. № 4. С. 505-535.

[37] В. Г. Кановей. К проблемам Н.Н. Лузина о существовании СА-множеств, не имеющих совершенных подмножеств // Матем. заметки. 1987. Т. 41. № 5. С. 750-759.

[38] В. Г. Кановей. Развитие дескриптивной теории множеств под влиянием трудов Н. Н. Лузина // УМН. 1985. Т. 40. № 3. С. 117-153.

[39] В. Г. Кановей. Идеи А. Н. Колмогорова в теории операций над множествами // УМН. 1988. Т. 43. №6. С. 93-128.

[40] В. Г. Кановей, В. А. Успенский. Проблемы Лузина о конституантах и их судьба // Вестник МГУ. Сер. матем., мех. 1983. №6. С. 73-87.

[41] A.S. Kechris. Measure and category in effective descriptive set theory // Ann. Math. Logic. 1973. V. 5. P. 337-384.

[42] A.S. Kechris. Classical Descriptive Set Theory. New York: Springer-Verlag, 1995.

[43] M. Kondô. Sur l'uniformisation des complémentaires analytiques et les ensembles projectifs de la seconde classe // Japan. J. Math. 1938. V. 15. P. 197-230.

[44] K. Kunen. Set Theory. An Introduction to Independence Proofs. Amsterdam: North-Holland, 1980. (Stud. Logic Found. Math. V. 102.)

[45] K. Kuratowski. Sur une généralisation de la notion de homéomorphie // Fund. Math. 1934. V. 22. P. 206-220.

[46] К. Куратовский. Топология. Т. 1. М.: Мир, 1970. 
[47] H.Lebesgue. Sur les fonctions représentables analytiquement // J. Math. Sér. 6. 1905. V. 1. P. $139-216$.

[48] H. Lebesgue. Préface // [57]. P. VII-XI. [Русский перевод: А. Лебег. Предисловие к книге Н.Н. Лузина "Лекции об аналитических множествах и их приложениях" / коммент. В. А. Успенского // УМН. 1985. Т. 40. № 3. С. 9-14.]

[49] A. Levy. Definability in axiomatic set theory. II // Mathematical Logic and Foundations of Set Theory. Amsterdam: North-Holland, 1970. P. 129-145.

[50] G. G. Lorentz. Who discovered analytic sets? // Math. Intelligencer. 2001. V. 23. № 4. P. 28-32.

[51] N. Lusin. Sur la classification de M. Baire // C. R. Acad. Sci. Paris. 1917. V. 164. P. 91-94. [Русский перевод: [60; с. 270-272].]

[52] N. Lusin. Sur les ensembles projectifs de M. Henri Lebesgue // C. R. Acad. Sci. Paris. 1925. V. 180. Р. 1572-1574. [Русский перевод: [60; с. 304-306].]

[53] N. Lusin. Les propriétés des ensembles projectifs // C. R. Acad. Sci. Paris. 1925. V. 180. Р. 1817-1819. [Русский перевод: [60; с. 307-308].]

[54] N. Lusin. Sur le problème de M. Émile Borel et la méthode des résolvantes // C. R. Acad. Sci. Paris. 1925. V. 181. Р. 279-281. [Русский перевод: [60; с. 312-314].]

[55] N. Lusin. Sur les ensembles analytiques // Fund. Math. 1927. V. 10. P. 1-95. [Русский перевод: [60; с. $380-459]$.

[56] N. Lusin. Sur le problème de M. J. Hadamard d'uniformisation des ensembles // Mathématica (Cluj). 1930. V. 4. P. 54-66.

[57] N. Lusin. Leçons sur les ensembles analytiques et leurs applications. Paris: Gauthier-Villars, 1930; 2nd corr. ed. New York: Chelsea, 1972. [Русский перевод: [60; с. 9-269]. Сокр. pyсc. пер.: Лекции об аналитических множествах и их приложениях. М.: Гостехиздат, 1953.]

[58] Н.Н. Лузин. Современное состояние теории функций действительного переменного. М.-Л.: ГТТИ, 1933; [60; с. 494-536].

[59] Н. Н. Лузин. О стационарных последовательностях // Труды физ.-матем. ин-та, отд. матем. 1934. Т. 5. С. $125-147$; [60; с. $642-661]$.

[60] Н. Н. Лузин. Собрание сочинений. Т. 2. М.: Изд-во АН СССР, 1958.

[61] N. Lusin, W. Sierpiński. Sur quelques propriétés des ensembles (A) // Bull. Int. Acad. Sci. Cracowie. 1918. V. 4. Р. 35-48. [Русский перевод: [60; с. 273-284].]

[62] N. Lusin, P. Novikoff. Choix effectif d'un point dans un complémentaire analytique arbitraire donné par un crible // Fund. Math. 1935. V. 25. Р. 559-560. [Два разных русских перевода: [60; с. 621-623] и [77; с. 49-50].]

[63] N. Lusin, W. Sierpiński. Sur un ensemble non mesurable B // J. Math. 1923. V. 2. P. 53-72. [Русский перевод: [60; с. 285-300].]

[64] В. А. Любецкий. Некоторые следствия гипотезы о несчетности множества конструктивных действительных чисел // Докл. АН СССР. 1968. Т. 182. № 4. С. 758-759.

[65] В. А. Любецкий. Из существования неизмеримого множества типа А 2 вытекает существование несчетного множества, не содержащего совершенного подмножества, типа СА // Докл. АН СССР. 1970. V. 195. № 3. Р. 548-550.

[66] В. А. Любецкий. Из существования неизмеримого множества типа А 2 следует существование несчетного множества типа СА, не содержашего совершенного подмножества // Тезисы докладов по алгебре, математической логике и вычислительной математике конференции пед. вузов центр. зоны РСФСР. Иваново, 1970. С. 22-24.

[67] В.А. Любецкий. Независимость некоторых предложений дескриптивной теории множеств от теории множеств Цермело-Френкеля // Вестник МГУ. Сер. матем., мех. 1971. № 2. С. 78-82. (Статья поступила в редакцию в январе 1970 г. - В. А. Любецкий.)

[68] В. А. Любецкий. Измеримость и наличие совершенного ядра у проективных множеств // Дисс. ... канд. физ.-матем. наук. М.: МГУ, 1971.

[69] В.А. Любецкий. Случайные последовательности чисел и $\mathrm{A}_{2}$-множества // Исследования по теории множеств и неклассическим логикам. М.: Наука, 1976. С. 96-122.

[70] В.А. Любецкий. Оценки и пучки. О некоторых вопросах нестандартного анализа // УМН. 1988. Т. 44. № 4. С. 99-153.

[71] В.И. Малыхин. Топология и форсинг // УМН. 1983. Т. 38. №1. С. 69-118.

[72] R. Mansfield. Perfect subsets of definable sets of real numbers // Pacific J. Math. 1970. V. 35. № 2. P. 451-457. 
[73] D. Martin, R. M. Solovay. Internal Cohen extensions // Ann. Math. Logic. 1970. V. 2. P. $143-178$

[74] A. R. D. Mathias. Surrealist landscape with figures (a survey of recent results in set theory) // Period. Math. Hungar. 1979. V. 10. № 2-3. P. 109-175. (Все наши ссылки на эту работу относятся к ее первоначальной версии, известной под названием: A survey of recent results in set theory. Stanford University, July 1968. Этот препринт содержит предварительные объявления о ранних результатах в теории множеств, за редкими исключениями без ссылок на публикацию и без (даже набросков) доказательств. Некоторые из приведенных там результатов цитируются в нашей статье.)

[75] J. Myhill, D. Scott. Ordinal definability // Proc. Sympos. Pure Math. 1971. V. 13. Part 1. P. 271-278.

[76] P. Novikoff. Sur la séparabilité des ensembles projectifs de seconde classe // Fund. Math. 1935. V. 25. Р. 459-466. [Русский перевод: [77; с. 43-48].]

[77] П. С. Новиков. Избранные труды: теория множеств и функций, математическая логика и алгебра. М.: Наука, 1979 .

[78] П. С. Новиков. О непротиворечивости некоторых положений дескриптивной теории множеств // Труды МИАН. 1951. Т. 38. С. 279-316; [77; c. 170-202].

[79] J. Raisonnier. A mathematical proof of S. Shelah's theorem on the measure problem and related results // Israel J. Math. 1984. V. 48. № 1. P. 48-56.

[80] J. Raisonnier, J. Stern. The strength of the measurability hypotheses // Israel J. Math. 1985. V. 50. № 4. P. 337-349.

[81] A. Roslanowski, S. Shelah. Norms on possibilities. II: more ccc ideals on $2^{\omega} / /$ J. Appl. Anal. 1997. V. 3. № 1. P. 103-127.

[82] G.E. Sacks. Forcing with perfect closed sets // Proc. Sympos. Pure Math. 1971. V. 13. Part 1. P. 331-355.

[83] E. Selivanowski. Sur les propriétés des constituantes des ensembles analytiques // Fund. Math. 1933. V. 21. P. 20-28.

[84] S. Shelah. Can you take Solovay's inaccessible away? // Israel J. Math. 1984. V. 48. №1. P. 1-47.

[85] J. Shoenfield. The problem of predicativity // Essays on the Foundations of Mathematics. Jerusalem: Magnes Press, Hebrew Univ., 1961. P. 132-139.

[86] Дж. Шенфилд. Математическая логика. М.: Наука, 1975.

[87] Дж. Шенфилд. Неразветвленное вынуждение // [86; с. 482-519].

[88] R. M. Solovay. On the cardinality of $\boldsymbol{\Sigma}_{2}^{1}$ sets of reals // Foundations of Mathematics. Symposium papers commemorating the 60th birthday of Kurt Gödel (Columbus, 1966). New York: Springer-Verlag, 1969. P. 58-73.

[89] R. M. Solovay. A model of set theory in which every set of reals is Lebesgue measurable // Ann. of Math. (2). 1970. V. 92. № 1. P. 1-56.

[90] R. M. Solovay, S. Tennenbaum. Iterated Cohen extensions and Suslin's problem // Ann. of Math. (2). 1971. V. 94. P. 201-245.

[91] M. Souslin. Sur une définition des ensembles mesurables B sans nombres transfinis // C. R. Acad. Sci. Paris. 1917. V. 164. P. 88-90.

[92] J. Stern. Generic extensions which do not add random reals // Methods in Mathematical Logic (Caracas 1983). Berlin: Springer-Verlag, 1985. P. 395-407. (Lecture Notes in Math. V. 1130.)

[93] J. Stern. On Lusin's restricted continuum problem // Ann. of Math. (2). 1984. V. 120. № 1. P. 7-37.

[94] J. Stern. Regularity properties of definable sets of reals // Ann. Pure Appl. Logic. 1985. V. 29. № 3. P. 289-324.

[95] В. М. Тихомиров. Открытие А-множеств // Историко-матем. исследования. 1993. № 34 . C. $129-139$.

[96] J. Truss. Sets having calibre $\aleph_{1} / /$ Logic Colloquium 76 (Oxford, 1976). Amsterdam: North-Holland, 1977. P. 595-612. (Stud. Logic Found. Math. V. 87.)

[97] В.А.Успенский. Вклад Н.Н. Лузина в дескриптивную теорию множеств и функций: понятия, проблемш, предсказания // УМН. 1985. Т. 40. № 3. С. 85-116. 